Prepared in cooperation with Commander, Navy Region Southwest

Monitoring Breeding and Migration of Neotropical Migratory Birds at Point Loma, San Diego County, California, 5-Year Summary, 2011-15

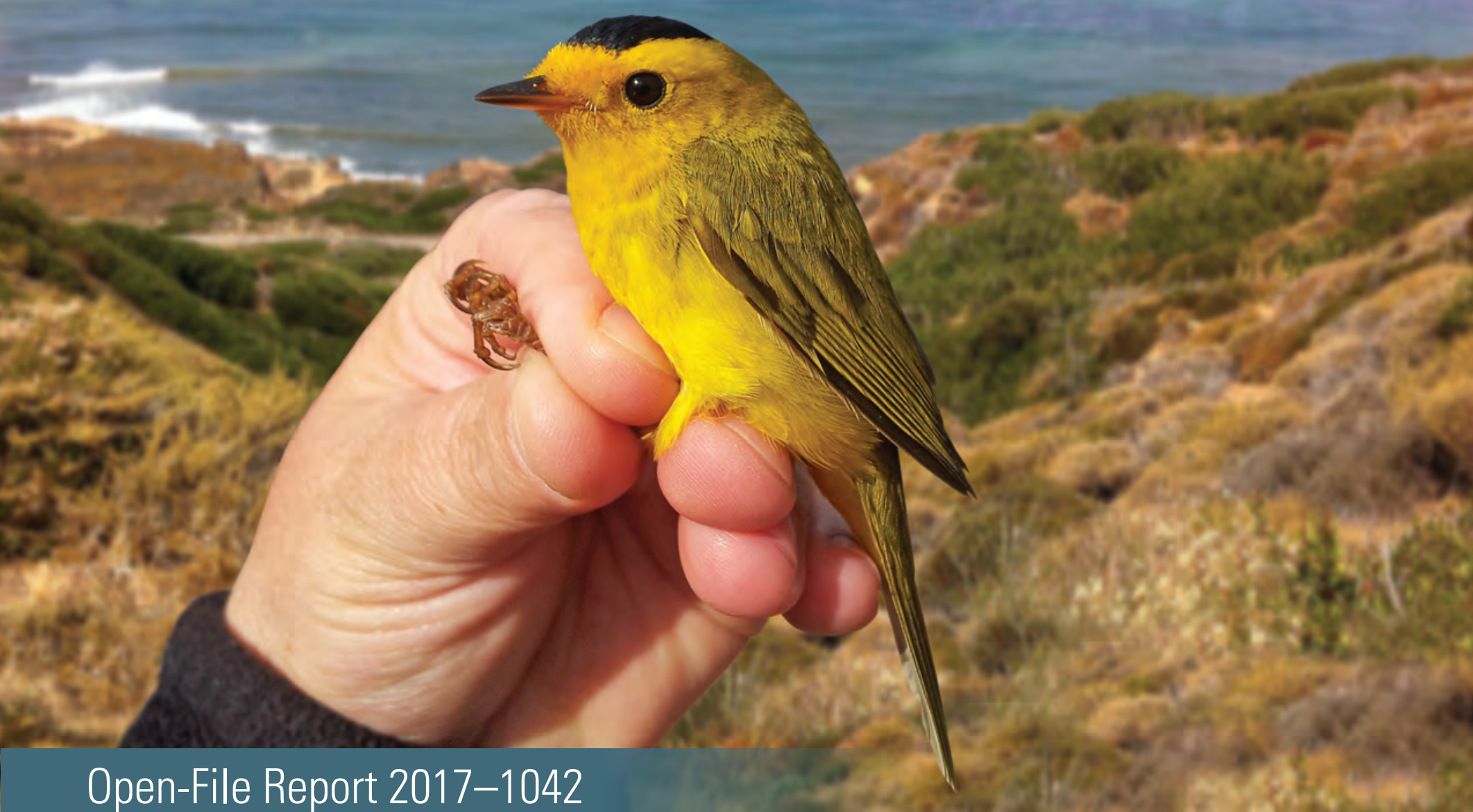

U.S. Department of the Interior

U.S. Geological Survey 
Cover: Photograph showing west-facing view from the banding station at Cabrillo National Monument, Point Loma, San Diego, California. Photograph by Melanie Madden, U.S. Geological Survey, 2011.

Photograph of male Wilson's warbler (Cardellina pusilla), by Scarlett Howell, U.S. Geological Survey, 2013. 


\section{Monitoring Breeding and Migration of Neotropical Migratory Birds at Point Loma, San Diego County, California, 5-Year Summary, 2011-15}

By Suellen Lynn, Melanie C. Madden, and Barbara E. Kus

Prepared in cooperation with Commander, Navy Region Southwest

Open-File Report 2017-1042

U.S. Department of the Interior

U.S. Geological Survey 


\section{U.S. Department of the Interior \\ RYAN K. ZINKE, Secretary}

\section{U.S. Geological Survey \\ William H. Werkheiser, Acting Director}

U.S. Geological Survey, Reston, Virginia: 2017

For more information on the USGS—-the Federal source for science about the Earth,

its natural and living resources, natural hazards, and the environment-visit

https://www.usgs.gov/ or call 1-888-ASK-USGS (1-888-275-8747).

For an overview of USGS information products, including maps, imagery, and publications, visit https:/store.usgs.gov/pubprod.

Any use of trade, firm, or product names is for descriptive purposes only and does not imply endorsement by the U.S. Government.

Although this information product, for the most part, is in the public domain, it also may contain copyrighted materials as noted in the text. Permission to reproduce copyrighted items must be secured from the copyright owner.

Suggested citation:

Lynn, Suellen, Madden, M.C., and Kus, B.E., 2017, Monitoring breeding and migration of neotropical migratory birds at Point Loma, San Diego County, California, 5-year summary, 2011-15: U.S. Geological Survey Open-File Report 2017-1042, 119 p., https://doi.org/10.3133/ofr20171042.

ISSN 2331-1258 (online) 


\section{Contents}

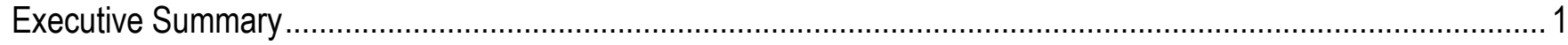

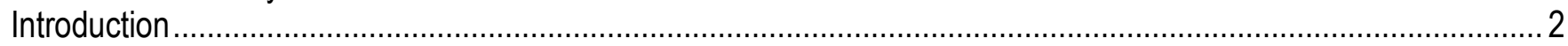

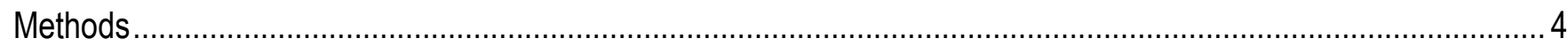

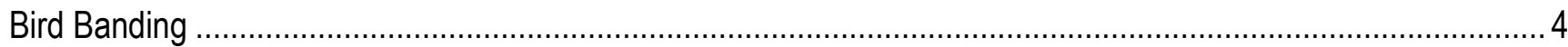

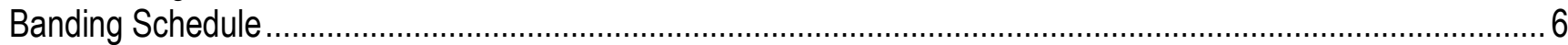

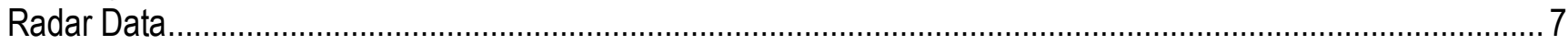

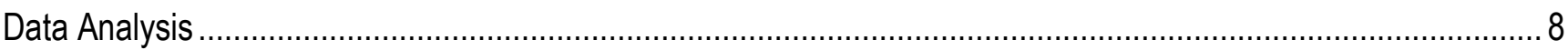

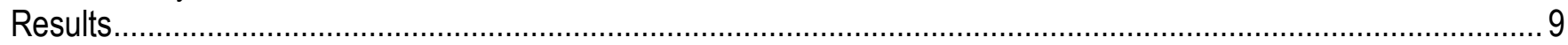

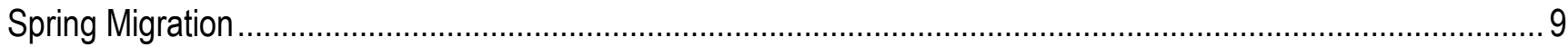

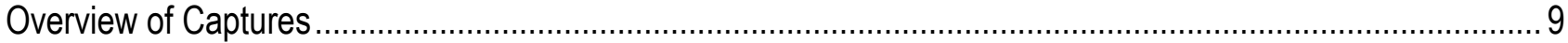

Capture Rates

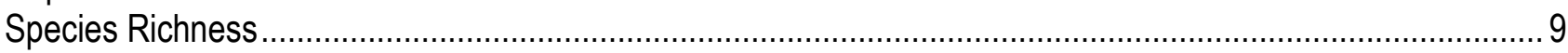

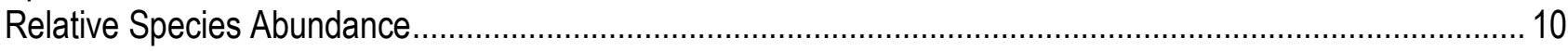

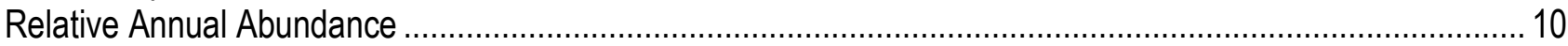

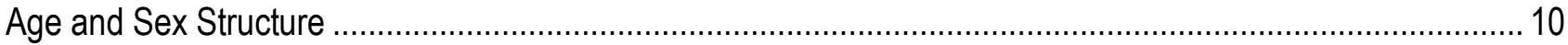

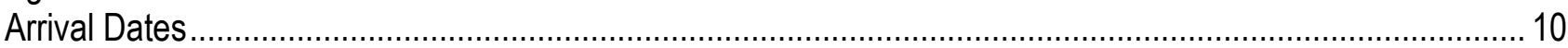

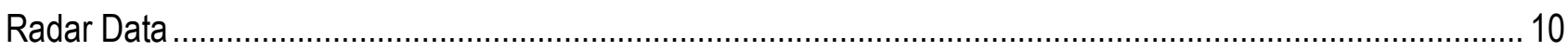

Monitoring Avian Productivity and Survivorship .................................................................................... 11

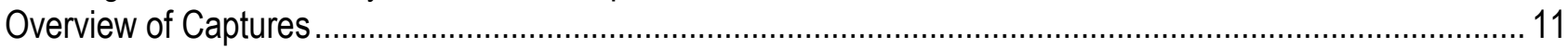

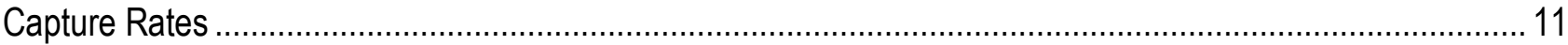

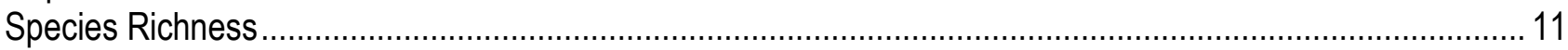

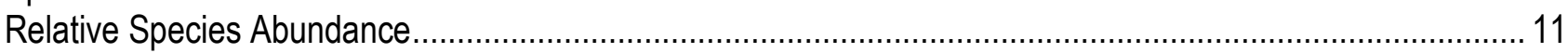

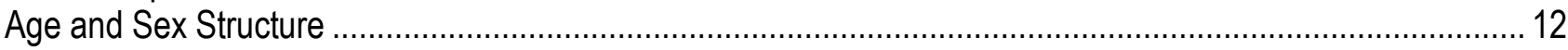

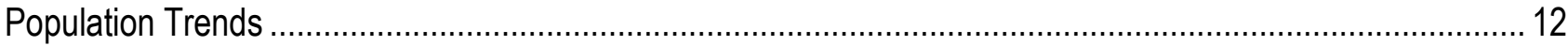

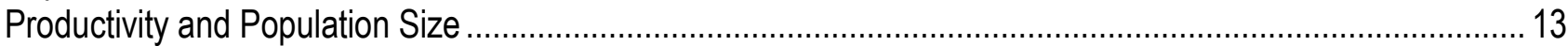

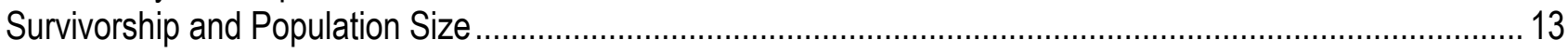

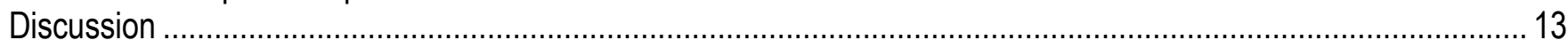

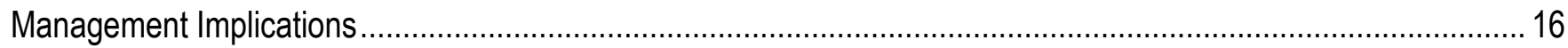

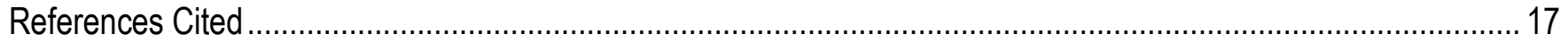

Appendix A. Alpha Codes, Common Names, and Scientific Names for Species Captured and Observed, Point Loma, San Diego, California, 2011-15 ............................................................................................... 19

Appendix B. Tables and Figures for Spring Migration 2011-15, Point Loma, California..................................... 23

Appendix C. Tables and Figures for Monitoring Avian Productivity and Survivorship Program, Point Loma, California, 2011-15. 


\section{Figures}

Figure 1. Map showing location of Point Loma bird banding station, San Diego, California 5

Figure 2. Representative NEXRAD images showing spring 2011-15 banding days with (A) low versus (B) high migrant bird capture rates, Point Loma banding station, San Diego, California

\section{Table}

Table 1. Locations of mist nets at the Point Loma bird banding station, San Diego, California

\section{Conversion Factors}

International System of Units to Inch/Pound

\begin{tabular}{|c|c|c|}
\hline Multiply & By & To obtain \\
\hline \multicolumn{3}{|c|}{ Length } \\
\hline millimeter (mm) & 0.03937 & inch (in.) \\
\hline meter $(\mathrm{m})$ & 3.281 & foot $(\mathrm{ft})$ \\
\hline kilometer (km) & 0.6214 & mile (mi) \\
\hline kilometer (km) & 0.5400 & mile, nautical (nmi) \\
\hline meter $(\mathrm{m})$ & 1.094 & yard (yd) \\
\hline \multicolumn{3}{|c|}{ Area } \\
\hline hectare (ha) & 2.471 & acre \\
\hline
\end{tabular}

\section{Datum}

Horizontal coordinate information is referenced to the World Geographic System of 1984 (WGS84).

\section{Abbreviations}

$\begin{array}{ll}\text { dBZ } & \text { decibels of reflectivity } \\ \text { MAPS } & \text { Monitoring Avian Productivity and Survivorship } \\ \text { NEXRAD } & \text { Next-Generation Radar } \\ \text { NOAA } & \text { National Oceanic and Atmospheric Administration } \\ \text { Z } & \text { reflectivity }\end{array}$




\section{Monitoring Breeding and Migration of Neotropical Migratory Birds at Point Loma, San Diego County, California, 5-Year Summary, 2011-15}

By Suellen Lynn, Melanie C. Madden, and Barbara E. Kus

\section{Executive Summary}

We operated a bird banding station on the Point Loma peninsula in western San Diego County, California, during spring and summer from 2011 to 2015. The station was established in 2010 as part of a long-term monitoring program for neotropical migratory birds during spring migration and for breeding birds as part of the Monitoring Avian Productivity and Survivorship (MAPS) program.

During spring migration (April and May), 2011-15, we captured 1,760 individual birds of 54 species, 91 percent $(1,595)$ of which were newly banded, fewer than 1 percent $(3)$ of which were recaptures that were banded in previous years, and 9 percent (143 hummingbirds, 2 hawks, and 17 other birds) of which we released unbanded. We observed an additional 22 species that were not captured. Thirty-four individuals were captured more than once. Bird capture rate averaged $0.49 \pm 0.07$ captures per net-hour (range 0.41-0.56). Species richness per day averaged $6.87 \pm 0.33$. Cardellina pusilla (Wilson's warbler) was the most abundant spring migrant captured, followed by Empidonax difficilis (Pacific-slope flycatcher), Vireo gilvus (warbling vireo), Zonotrichia leucophrys (white-crowned sparrow), and Selasphorus rufus (rufous hummingbird). Captures of white-crowned sparrow decreased, and captures of Pacific-slope flycatcher increased, over the 5 years of our study. Fifty-six percent of known-sex individuals were male and 44 percent were female. The peak number of new species arriving per day ranged from April 1 (2013-six species) to April 16 (2012-five species). A significant correlation was determined between the number of migrants captured each day per net-hour and the density of echoes on the Next-Generation Radar (NEXRAD) images across all 5 years, and in each year except 2014. NEXRAD radar imagery appears to be a useful tool for detecting pulses in migration.

Our results indicate that Point Loma provides stopover habitat during migration for 76 migratory species, including 20 species of conservation concern. Two of these species, Vireo bellii pusillus (least Bell's vireo) and Empidonax traillii (willow flycatcher) are listed as State and (or) federally threatened or endangered.

Except for Archilochus alexandri (black-chinned hummingbird) and Setophaga occidentalis (hermit warbler), which arrived later during the migratory season in latter years of our study, median arrival dates for migratory species tended to be earlier each year or did not change across 5 years. Of the five most common migratory species, white-crowned sparrow and rufous hummingbird arrived earlier in latter years of the study, but Pacific-slope flycatcher, warbling vireo, and Wilson's warbler median arrival dates were variable and showed no trend. 
We captured 1,680 individuals of 66 species during the MAPS/breeding season (May through August) across the 5 years of our study, 72 percent $(1,211)$ of which were newly banded, 10 percent (167) of which were recaptures, and 18 percent (302 hummingbirds and other birds that escaped prior to banding) of which we released unbanded. Bird capture rate averaged $0.65 \pm 0.21$ captures per net-hour (range 0.12-2.54). Species richness per day ranged from $9.80 \pm 5.01$ to $14.20 \pm 4.57$. Calypte anna (Anna's hummingbird) was the most abundant breeding species captured, followed by Oreothlypis celata (orange-crowned warbler), Psaltriparus minimus (bushtit), Pipilo maculatus (spotted towhee), Thryomanes bewickii (Bewick's wren), Melozone crissalis (California towhee), and Chamaea fasciata (wrentit). Fifty-one percent of known-sex captures were female, and 49 percent were male. Thirty-one percent of known-age captures were juveniles.

Populations of bushtits and orange-crowned warbler decreased significantly over 5 years. Anna's hummingbird abundance was high for 4 years, and then decreased in 2015. Bewick's wren and wrentit populations were highest in 2015. There was no obvious pattern in spotted towhee and California towhee abundance across 5 years. Annual breeding productivity for most species was low in 2014 and high in 2015. Bewick's wren had the highest breeding productivity of the six most commonly captured species, followed by bushtit. Orange-crowned warbler had the lowest breeding productivity. Breeding productivity was a significant predictor of population size the next year for bushtit, but not for any other resident breeding species examined.

Adult survivorship was generally high from 2013 to 14, and low from 2014 to 15 . Wrentits had the highest survivorship of the most common species captured, followed by California towhee and orange-crowned warbler. Adult survivorship was lowest for bushtits and spotted towhees. Adult survivorship was a significant predictor of population size for bushtits, but not for any other resident species examined.

Our monitoring results indicate that Point Loma provides breeding habitat for seven species of conservation concern. One of these species, the federally threatened Polioptila californica californica (California gnatcatcher), was documented breeding at the study site.

\section{Introduction}

Monitoring Avian Productivity and Survivorship (MAPS; Institute for Bird Populations, Point Reyes Station, California) is an international monitoring program that uses bird capture and banding data to compile basic demographic parameters of migratory species, many of which are imperiled regionally and even globally. Age- and sex-specific data on annual survival, reproduction, and recruitment can be gathered and compared across stations to identify population trends for species of interest and can be used to identify proximate factors responsible for trends, negative trends in particular. In turn, information obtained from long-term monitoring of bird populations can be used to guide management activities intended to maintain or re-establish viable populations throughout the species' ranges.

We established a MAPS station on the Point Loma peninsula in western San Diego County, California, in 2010. This station was established as part of a long-term monitoring program for neotropical migratory birds for Naval Base Point Loma (U.S. Department of Defense) and Cabrillo National Monument (U.S. Department of the Interior, National Park Service) and was operated in a manner consistent with other banding stations participating in an effort to monitor birds worldwide. The station was originally established to monitor both autumn and spring migration, but initial results indicated autumn captures were not sufficient to continue autumn monitoring. 
Understanding the factors that affect migratory bird populations during all phases of their life cycle is critical to species and ecosystem conservation planning. Bird migration studies are useful because they apply to species that occupy sites across a broad geographic range and, potentially, a broad range of habitat types, during the different phases of their life cycles. Because migration provides a link between wintering and breeding phases of the annual cycle, migration studies can elucidate the responses of species to diverse pressures affecting any of the phases of their annual cycles, including habitat alteration and loss, diseases, environmental contaminants, and global climate change.

Studying birds during migration can also inform land managers about local factors that affect migratory stopover habitat. High-quality migratory stopover habitat provides abundant resources that allow individuals to re-establish energy reserves in the short time period available for completing migration (Skagen and others, 2004). Identifying these stopover sites is the first step in conserving and potentially enhancing habitats that benefit bird populations.

Methods of studying migration can include traditional auditory or visual surveys, examination of museum specimens, constant-effort mist netting and banding, radio-telemetry (or satellite tracking), and more recently, radar tracking (Lincoln and others, 1998). Each method of study provides benefits for the efficient collection of specific data, and selection of the method depends on the data required.

Radar has been used in the study of bird migration for more than 60 years, since the discovery in the early 1940s that birds were responsible for some of the echoes ("angels" or "ghosts") displayed on surveillance radar (Lack and Varley, 1945). Radar is a tool that can provide information on bird movements for those species that are difficult to examine directly because they migrate at night or at high elevations and over large spatial extents (Diehl and Larkin, 2004; O'Neal and others, 2010). Weather surveillance radar is especially useful because, when correlated with mist netting or other forms of direct observation of migrating birds, it can provide quantitative information on a broad geographic scale. The Weather Surveillance Radar-1998 Doppler or Next-Generation Radar (NEXRAD) is operated at 154 stations across the United States; archived imagery and metadata from NEXRAD are available from the National Oceanic and Atmospheric Administration (NOAA) on-line. For our study, we correlated the capture rate of migratory passerines from our banding station at Naval Base Point Loma and Cabrillo National Monument with NEXRAD data in spring 2011-15.

This report summarizes 5 years of station operation from 2011 to 2015. Five years is the minimum consecutive years necessary to obtain reliable productivity indices and survivorship estimates described in the MAPS program (DeSante and others, 2011). There are five objectives of this project: (1) to obtain an index of sizes and trends of various neotropical migratory bird species populations, (2) to estimate demographic parameters for neotropical migratory bird species, (3) to estimate annual productivity for these species, (4) to augment existing distributional information for "sensitive" avian species, and (5) to evaluate the utility of using NEXRAD data to describe patterns of spring bird migration at Point Loma. 


\section{Methods}

\section{Bird Banding}

We established a bird banding station (Point Loma) on property managed by Naval Base Point Loma and Cabrillo National Monument in autumn 2010. The Point Loma peninsula (approximately 10 $\mathrm{km}$ long) runs north-south and forms the northwestern boundary of San Diego Bay (fig. 1). Most of the peninsula is owned by the U.S. Navy, but the National Park Service administers approximately 113 ha near the southern tip. Vegetation at the station is a mix of maritime succulent scrub, Diegan coastal sage scrub, and southern maritime chaparral, and typical woody species include Rhus integrifolia (lemonade berry), Heteromeles arbutifolia (toyon), and Artemisia californica (California sagebrush). The Point Loma station overlaps a bird banding station that operated at Cabrillo National Monument from May 2006 through August 2010 (Institute for Bird Populations, unpub. data, 2006-2010)).

Bird banding at the Point Loma banding station followed standardized MAPS protocol (DeSante and others, 2011), with modifications for migration. Ten mist nets, placed 85-400 m apart, were erected in fixed locations selected for their potential to capture birds moving through vegetation (fig. 1, table 1). Mist nets were made of $30 \mathrm{~mm}$ mesh black nylon and were $12 \mathrm{~m}$ long $\times 2.6 \mathrm{~m}$ high, with four trammels ("pockets") running the length of the net. In two net lanes, we used two 6-m nets where necessary to "bend" nets around existing vegetation. Nets were suspended from vertical aluminum poles anchored by permanent rebar stakes and covered a vertical area approximately $0.25-2.5 \mathrm{~m}$ above the ground. Nets were opened within 30 minutes of dawn and remained open for 5 hours during the breeding season, typically closing between 1100 and 1200 PST, and 3 hours during migration, typically closing between 0900 and 1000. Nets were not operated during inclement weather such as strong wind, rain, extreme heat, or cold. Dates of net operation are detailed in section, "Banding Schedule." 


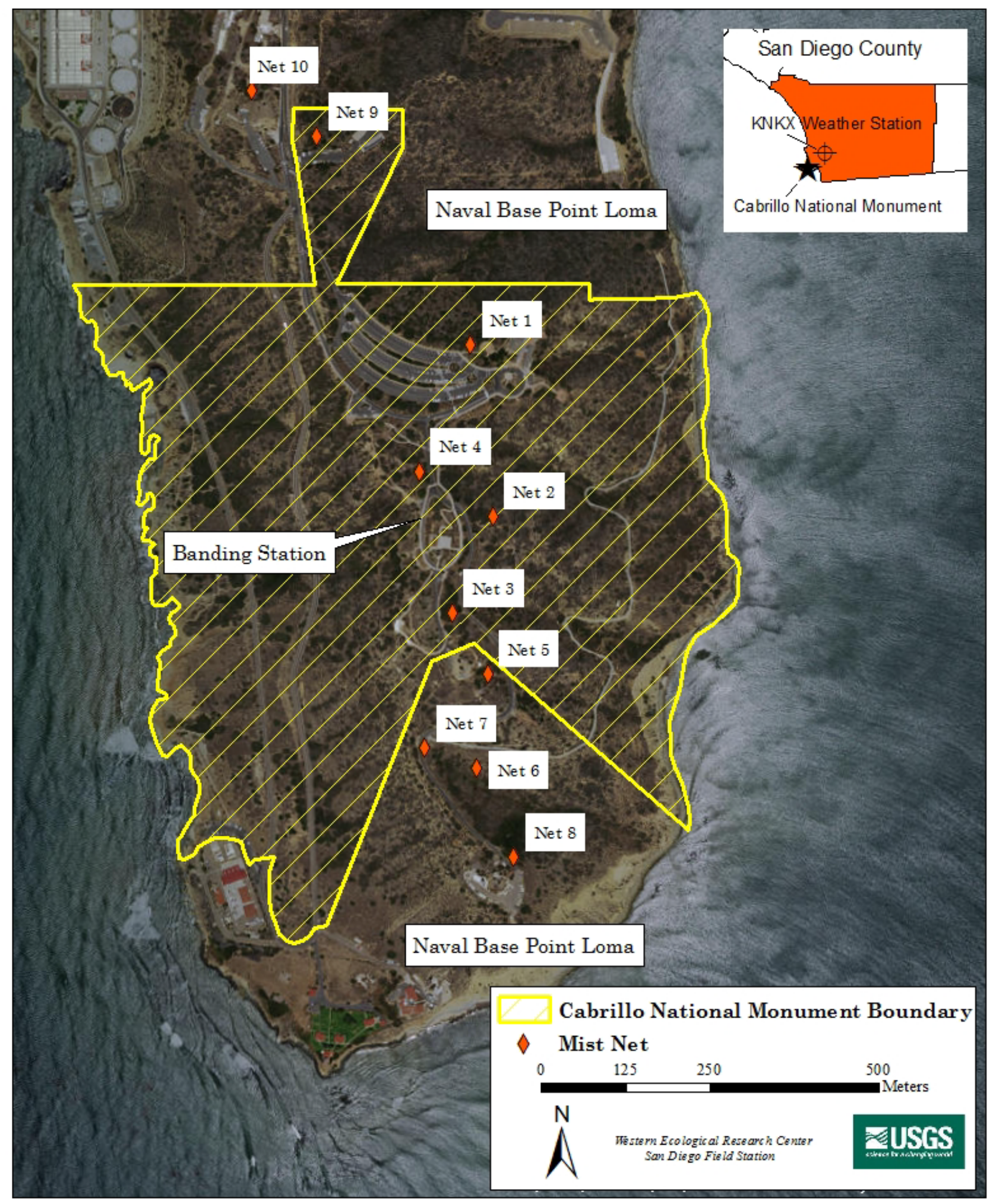

Figure 1. Map showing location of Point Loma bird banding station, San Diego County, California. 
Table 1. Locations of mist nets at the Point Loma bird banding station, San Diego County, California.

[Coordinates are in World Geographic System of 1984]

\begin{tabular}{ccc}
\hline Net No. & Longitude & Latitude \\
\hline 1 & -117.24042 & 32.67466 \\
2 & -117.24005 & 32.67236 \\
3 & -117.24069 & 32.67106 \\
4 & -117.24122 & 32.67296 \\
5 & -117.24014 & 32.67026 \\
6 & -117.24030 & 32.66899 \\
7 & -117.24112 & 32.66926 \\
8 & -117.23971 & 32.66780 \\
9 & -117.24286 & 32.67743 \\
10 & -117.24389 & 32.67804 \\
\hline
\end{tabular}

Nets were checked every 30-40 minutes by observers working circuits. Hummingbirds, upland game birds, and raptors were not banded but were identified to species, age, and sex when possible and released immediately at the capture site. All other birds were removed from nets, held in cloth bags labeled with the net number, and taken to a central processing location where they were banded with Federal numbered aluminum bands. Data recorded for each individual caught included age, sex, skull pneumatization, breeding condition, weight, wing chord, fat deposition, feather wear, and molt status, using Pyle (1997) as a reference. Birds were held for 5-60 minutes depending on the number of birds captured during one net run. After processing, juveniles, brooding females, and resident birds from the more distant nets were released near the net in which they had been captured, whereas all other birds were released at the processing station. All species captured and (or) observed at the site were recorded in a daily list to help determine breeding status and to document species that were present at the station, but were not captured. Typically, four to five personnel operated the station.

\section{Banding Schedule}

Because we were interested in examining both breeding and migratory activity at the Point Loma banding station, we operated mist nets during two seasons throughout the 5 years of the project: (1) spring (migration) and (2) MAPS (breeding).

In spring, the station was operated every weekday, weather permitting, from April 1 to 30, and 3 days during May (once every 10 days corresponding to the first three MAPS banding periods), for a total of 22-23 days each year. During the MAPS season, the banding station was operated 1 day during every 10-day period between May 5 and August 7, following standard MAPS protocol (10 days total). Migration continued after the end of April; thus we included the 3 banding days in May in both migratory and breeding season analyses. We restricted our analyses of spring migration to migratory birds (that is, species that do not breed at Point Loma). 


\section{Radar Data}

We retrieved archived Level II NEXRAD imagery for San Diego (from San Diego, Miramar MCAS/Mitscher Field Airport weather station KNKX; fig. 1) from the NOAA Satellite and Information Service website (http://www.ncdc.noaa.gov/nexradinv/). Level II data include reflectivity (a measure of radar echo intensity determined by the density and size of targets) and radial velocity (measured by Doppler echoes indicating direction and speed of the targets relative to the radar). NEXRAD radar sweeps a conical zone centered on the weather station at increasing angles relative to the ground, beginning at 0.5 degrees and increasing to approximately 20 degrees under certain conditions. We used images collected at the lowest angle available during our trapping period ( 0.57 degrees $)$, which is most useful for bird migration studies because it detects low-flying targets (that is, migrating passerines) at a greater distance than higher angle sweeps (Able, 1970).

We selected and downloaded one sweep image for each evening prior to banding that was closest to 2.5 hours post-sunset. This image was standardized based on prior years' analyses and captures over-flight approximately 2 hours post-exodus (when most nocturnal migrants take off; Able, 1970). NEXRAD imagery typically shows a characteristic doughnut shaped "bloom" that begins at exodus when nocturnal migrants lift off and enter the radar beam. As more birds enter the beam and begin to move, the bloom intensifies to a peak when the density of birds flying overhead is highest (peak over-flight; fig. 2). This pattern has been identified and used in nocturnal migratory bird studies in the Eastern and Midwestern United States where open topography means fewer buildings or mountains that can block the radar beam, creating clutter and obscuring migratory patterns on the radar imagery.

Because San Diego is located in a basin with mountains directly east, radar imagery indicating nocturnal migration is likely to be most useful to the west (over the ocean) and along the coastal plain to the northwest and south. Therefore, we limited our inspection of radar imagery to these areas where clutter was minimized.

We used the Weather and Climate Toolkit (National Oceanic and Atmospheric Administration, 2016) to view NEXRAD imagery. We inspected NEXRAD images from dates when we knew migration was not occurring (December) to verify that the images during migration were not a product of topographical or other types of permanent scatter. We also examined images from foggy days to verify that fog did not create an echo pattern resembling migratory birds.
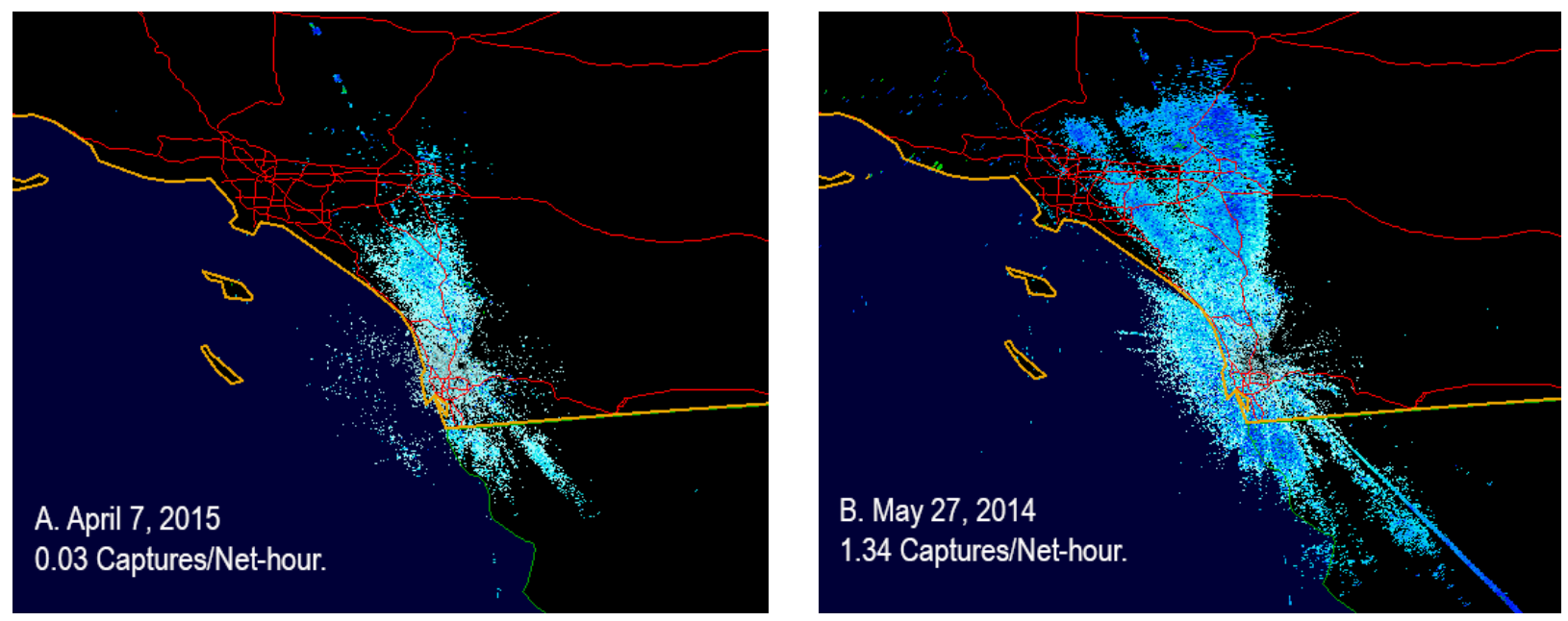

Figure 2. Representative Next-Generation Radar images showing spring 2011-15 banding days with (A) low and (B) high migrant bird capture rates, Point Loma banding station, San Diego County, California. 
Our objective was to detect migration through NEXRAD data in our region and was not quantitative; therefore, we did not attempt to correct for other organisms (for example, bats) that may have been included in the imagery.

We quantified the density of echoes for each image with geographic analysis. The selected reflectivity images were imported into ArcMAP 10.1 (ESRI Inc., 1999-2012 ${ }^{\circledR}$ ) and trimmed to a radius of $100 \mathrm{~km}$ from the center of the radar. Any obvious storm clouds were filtered from the images. The reflectivity data were recorded in logarithmic units known as decibels of reflectivity (dBZ), which we converted to linear reflectivity (Z) for analysis (Randall and others, 2011) for each echo in the $100 \mathrm{~km}$ radius circle. We multiplied $Z$ by the area of each pixel it represented and then summed $Z *$ Area across all echoes for each $100-\mathrm{km}$ radius circle to generate a single number representing the total reflectivity in the circle.

\section{Data Analysis}

Bird captures were quantified by species, age, sex, and number of captures for spring migration and for MAPS. We used two categories of bird captures for our analyses: (1) total number of captures (includes all newly banded, recaptured, and unbanded birds) and (2) total number of individuals captured (includes only newly banded birds, unbanded birds, and first recaptures of birds banded during previous activities at the site). We used only the initial capture of a bird on each banding day in analyses (we did not record same day recaptures). A bird was considered a recapture on each unique day it was captured after its original banding. We also treated unbanded captures as separate individuals with the caveat that there is a slight possibility these individuals were recaptured without us knowing, but the likelihood of recapturing any individual was low. We used the total number of captures each year to calculate the overall capture rates per net and the overall capture rate of a species per 100 net-hours each year. Captures per 100 net-hours is a standard measurement used for bird banding data. We then calculated the average total number of captures per year and captures per 100 net-hours over the 5 years of the study. We used the total number of individuals to calculate: (1) species richness (the number of species captured at the site), (2) relative species abundance (the proportion of all individuals represented by a particular species), (3) sex and age ratios (to determine the structure of migrating and breeding populations), and (4) first and median arrival dates for each species during migration. First and median arrival dates can be used to track possible future changes in the timing of migration associated with climate change.

Neotropical migratory species were defined as those covered under the Neotropical Migratory Bird Conservation Act of 2000 (U.S. Fish and Wildlife Service, 2011). Migratory birds were then categorized as breeding or migrant, according to behavior, breeding condition, and breeding status records for the site (not for the region as a whole). Based on this categorization, neotropical migratory species that were captured only during migration and are not known to breed at the site, such as common yellowthroat (latin names of bird species presented in appendix A, tables A1 and A2), were considered migrants even though they may breed nearby and be year-round residents of the region. Breeding or migratory status for some species changed during the 5 years of the project according to new data collected each year. For instance, Costa's hummingbird was considered a migrant until 2014, when it was considered a breeding species because of the frequency of its capture (captured throughout May) during the breeding season. Only consistently non-breeding, neotropical migratory species were included in our analyses of spring migration and radar data. For instance, both breeding and migratory subspecies of orange-crowned warbler were captured at the Point Loma banding station; therefore, they were not included in the migration analysis. 
We calculated the number of migratory birds captured per net-hour for each banding day in April (19-20 days) and May (3 days) for the 5 years of our project. We calculated Pearson's productmoment correlation coefficient by correlating the number of migrant captures per net-hour with the total reflectivity within $100 \mathrm{~km}$ of the radar.

We calculated Pearson's product-moment correlation coefficient to determine if capture rates for the most common migratory and breeding species were associated with year. We used simple regression to determine if observed population size of each of the six most common breeding species was affected by the previous year's breeding productivity and also adult survivorship from the previous year. Correlations and regressions were considered significant if $P \leq 0.10$.

\section{Results}

\section{Spring Migration}

\section{Overview of Captures}

In 3,689:50 net-hours during spring migration between 2011 and 2015, we had a total of 1,796 migrant captures (appendix B, tables B1-B6). Of these, 1,595 (89 percent) were newly banded during spring migration, 3 ( $<1$ percent) were recaptures that were banded in previous years, and 162 (9 percent) were released unbanded (143 hummingbirds, 2 hawks, and 17 other birds that were released without banding), for a total of 1,760 individuals captured (appendix B, tables B1, B7-B11). Ninety-eight percent of banded individuals $(1,563 / 1,598 ; 38$ species $)$ were captured only once in a year during spring migration, and 2 percent (34/1,598; 10 species) were captured twice (appendix B, tables B7-B11). We captured 54 species (appendix B, tables B12-B16, B22; unidentified species not included in species total). Additional species (22 migrants and 8 residents) were observed at the banding station, but not captured (appendix A, table A2).

Three individuals were recaptured in 2014 that were originally banded in previous years. One of these, a hermit thrush, was originally banded in southern Oregon on September 18, 2012 and was recaptured at Point Loma banding station on April 7, 2014. The other two individuals, a house wren and a ruby-crowned kinglet, were originally banded at the Point Loma banding station in 2013 and recaptured in April 2014. The house wren was originally banded as a juvenile on June 25, 2013, likely dispersing from its natal area. The ruby-crowned kinglet was captured on almost the same day both years (April 3, 2013; April 4, 2014), was captured in the same net both years (Net 9), and was captured at virtually the same time of day both years (0910 in 2013 and 0900 in 2014).

\section{Capture Rates}

Over the 5 years of our study, spring capture rates averaged $49 \pm 7$ per 100 net-hours for all nets combined (range 41-56 captures per 100 net-hours) (appendix B, tables B2-B6). Capture rates by net ranged from 35 to 77 captures per 100 net-hours, with Nets 4 and 9 being the most productive (appendix B, tables B2-B6).

\section{Species Richness}

The number of spring migrant species captured per day ranged from 0-16 (appendix $\mathrm{B}$, tables B12-B16). Species richness among captures was highest in the third week of April and the beginning of May. Overall species richness for spring averaged $6.87 \pm 0.33$ per day over the 5 years of our study. 


\section{Relative Species Abundance}

Wilson's warbler was the most abundant spring migrant species, with 364 individual captures and 10.07 total captures per 100 net-hours (appendix B, figs. B1-B5; tables B1 and B12-B16). The second most abundant species was Pacific-slope flycatcher, with 283 individuals ( 7.83 total captures per 100 net-hours), followed by warbling vireo with 177 individuals captured (4.90 total captures per 100 net-hours). Additional species accounting for at least 5 percent of all individuals captured include whitecrowned sparrow (146) and rufous hummingbird (99). Together, these five species accounted for 61 percent $(1,069 / 1,760)$ of all spring migrant individuals captured.

\section{Relative Annual Abundance}

Of the five most commonly captured migratory species described above, three (rufous hummingbird, warbling vireo, and Wilson's warbler) showed variable abundance during the 5 years of our study (appendix B, figs. B1-B5). Capture rates for one species, white-crowned sparrow, decreased significantly from 2011 to $2015(r=-0.83, P=0.04, \mathrm{df}=4)$. Conversely, capture rates for Pacific-slope flycatcher increased from 2011 to $2015(r=0.77, P=0.07, \mathrm{df}=4)$.

\section{Age and Sex Structure}

Over the 5 years of our study, 56 percent (439/783) of known-sex captures during spring migration were male and 44 percent (344/783) were female (appendix B, tables B17-B21). Because only migrant species were included in the analyses and banding occurred prior to the breeding season of most migrants, only three juveniles were captured, two black-chinned hummingbirds (May 15, 2014 and April 27, 2015) and one Say's phoebe (April 28, 2015). The timing of juvenile captures for both of these species was unusually early but still within the range of estimated dates for post-fledging dispersal, based on early breeding records of both species (Baltosser and Russell, 2000; Schukman and Wolf, 1998).

\section{Arrival Dates}

The peak number of new species arriving (captured) per day ranged in date from April 1 (in 2013) to April 16 (in 2012). Except for 2014, new species arrived at a steady rate throughout April (appendix B, figs. B6-B11, table B22). In 2014, most new migrant species arrived during the first half of April. Migration was earliest in 2013 (average median and first arrival dates were earliest; April 12 and 20, respectively) and latest in 2011 (April 19 and 28, respectively). Of species with more than one individual captured across years, hermit thrush had the earliest first arrival date and western woodpewee had the latest first arrival date, averaged across years. Blue-gray gnatcatcher had the earliest median arrival date and western wood-pewee had the latest median arrival date, averaged across years.

\section{Radar Data}

We included 22 days from spring 2011, 22 days from 2012, 19 days from 2013, 22 days from 2014, and 19 days from 2015 in our analysis (appendix B, tables B2-B6). Radar data from 1 day in 2011, 3 days in 2013, 1 day in 2014, and 4 days in 2015 were excluded because precipitation images obscured possible over-flight images. The number of migratory birds captured per net-hour in the spring was significantly correlated with the total reflectivity within $100 \mathrm{~km}$ of the radar across all 5 years $(r=$ $0.47, P<0.01$; appendix B, fig. B12), and also by year in $2011(r=0.66, P=0.001)$, in $2012(r=0.38$, $P=0.08)$, in $2013(r=0.52, P=0.01)$, and in $2015(r=0.54, P=0.02)$, but not in $2014(r=0.16, P=$ 0.48 ; appendix B, fig. B13). 


\section{Monitoring Avian Productivity and Survivorship}

\section{Overview of Captures}

In 2,484:10 net-hours during the 2011-15 breeding seasons, we had a total of 1,737 captures (appendix C, tables $\mathrm{C} 1$ and $\mathrm{C} 3-\mathrm{C} 7$ ). The total number of captures per year averaged $347 \pm 85$, and ranged from 267 (2013) to 490 (2011). Of the 1,737 total captures, 1,211 (70 percent) were newly banded during the MAPS seasons (appendix C, table $\mathrm{C} 1), 167$ (10 percent) were first time recaptures that year (appendix $\mathrm{C}$, table $\mathrm{C} 2$ ), and 302 (17 percent) were released unbanded (hummingbirds and additional birds that escaped prior to banding), for a total of 1,680 individuals captured (appendix $\mathrm{C}$, tables C8-C12). The average number of new captures per year was $242 \pm 66$ (range 166 [2013]-349 [2011]; appendix C, table C1). Ninety-six percent of banded individuals $(1,328 / 1,378)$ were captured only once during the MAPS season, 3 percent $(46 / 1,378)$ were captured twice, and fewer than 1 percent $(4 / 1,378)$ were captured three times (appendix C, tables C8-C12). We captured 67 species (appendix C, table $\mathrm{C} 1$; unidentified species not included in species total), averaging $36 \pm 5$ species per year (range 32 [2012]-45 [2011]). Three State and (or) Federally listed species, California gnatcatcher (2 individuals), least Bell's vireo (3 individuals), and willow flycatcher (12 individuals) were captured during the MAPS periods from 2011 to 2015. Twenty-nine additional species were observed but not captured (appendix A, table A2).

\section{Capture Rates}

Over 5 years, MAPS capture rates averaged $65 \pm 21$ per 100 net-hours for all nets combined (appendix C, tables C3-C7). Capture rate was highest in 2011 (101 captures per 100 net-hours) and lowest in 2013 ( 54 captures per 100 net-hours). Across 5 years, capture rates by net ranged from 19 to 151 captures per 100 net-hours with Nets 1, 4, 8, and 10 being the most productive (appendix C, tables C3-C7).

\section{Species Richness}

Over the 5 years of our project, the number of species captured per day ranged from 5 to 22 for the MAPS banding effort (4 to 10 for species that breed at the banding station; appendix C, tables C13C17). Species richness among captures was highest in mid-May. Overall species richness averaged highest in 2011 (14.2 \pm 4.6 per day, $9.7 \pm 1.5$ per day for species that breed at the banding station) and lowest in 2015 ( $9.8 \pm 5.0$ per day, but 2014 (6.3 \pm 2.1 per day) for species that breed at the banding station.

\section{Relative Species Abundance}

Anna's hummingbird was the most abundant breeding MAPS species captured over 5 years, with 211 individual captures and 8.49 total captures per 100 net-hours (appendix C, tables C1, C13C17, figs. C1-C5). We did not band hummingbirds at this station, though we clipped the tip of one tail feather to determine whether or not a hummingbird had been captured previously. Only two Anna's hummingbirds were recaptured with clipped tail feathers (one in 2014 and one in 2015), so we assume the number of Anna's hummingbirds captured is a fair representation of the actual number of individuals. The second most abundant breeding species was orange-crowned warbler with 158 individuals (6.72 total captures per 100 net-hours). Bushtit was the third most abundant breeding species with 154 individuals (6.96 total captures per 100 net-hours). Additional breeding species accounting for at least 5 percent of all individuals captured in any one year include spotted towhee (102 individuals 
captured), Bewick's wren (86 individuals captured), California towhee (77 individuals captured), and wrentit (76 individuals captured). Together, all seven species accounted for 51 percent $(864 / 1,680)$ of all MAPS individuals captured.

Because of the overlap in our spring and MAPS banding dates and the occurrence of late spring migrants, some of the most abundant passage neotropical migratory species (those that do not breed onsite) in our spring migration analysis also were some of the most abundant species during MAPS. The most abundant passage neotropical migratory species captured during MAPS was Pacific-slope flycatcher with 162 individuals (6.52 total captures per 100 net-hours), accounting for 9.6 percent of all MAPS individuals captured. The second most abundant passage neotropical migratory species captured during MAPS was Wilson's warbler (151 individuals, 6.08 total captures per 100 net-hours, 9.0 percent of MAPS individuals captured) followed by warbling vireo (101 individuals captured, 4.15 total captures per 100 net-hours, 6.0 percent of MAPS individuals captured).

\section{Age and Sex Structure}

Fifty-one percent (413/805) of known-sex captures during MAPS were female, and 49 percent (392/805) were male (appendix C, tables C18-C22). Juveniles comprised 31 percent $(503 / 1,621)$ of the known-age population. The proportion of juveniles captured varied annually, with the lowest proportion of juveniles captured in $2014(0.19)$ and the highest proportion of juveniles captured in $2013(0.42)$. Juveniles of 30 species were captured, with Anna's hummingbird contributing 26 percent (132/503), followed by bushtit (15 percent [77/503]), Bewick's wren (10 percent [50/503]), spotted towhee (9 percent [45/503]), orange-crowned warbler (7 percent [35/503]), wrentit (7 percent [34/503]), and California towhee ( 7 percent [33/503]). Together, these seven species accounted for 81 percent (406/503) of all hatch-year individuals captured during MAPS.

\section{Population Trends}

Population trends of the seven most abundant species captured during MAPS activities, as measured by the number of adult individuals captured per 100 net-hours, varied annually and seasonally. Captures of individual bushtits and orange-crowned warblers decreased significantly over the 5 years of our project (appendix C, tables C13-C17; fig. C6; $r=-0.91, P=0.03 ; r=-0.80, P=0.10$ respectively). Captures of Anna's hummingbird were high for 4 years (46-56 individuals), then decreased considerably in 2015 (8 individuals). Conversely, individual captures of Bewick's wren and wrentits were highest in 2015. Individual captures of spotted towhee and California towhee varied by year but not with an obvious pattern.

Individual captures of Anna's hummingbird and orange-crowned warbler significantly decreased throughout the MAPS banding season (appendix $\mathrm{C}$, tables $\mathrm{C} 13-\mathrm{C} 17$; fig. $\mathrm{C} 7 \mathrm{~A}$ and $\mathrm{E} ; r=-0.90, P=<$ $0.001 ; r=-0.82, P<0.01$ respectively), likely because some individuals of these species were migrants and therefore were captured only during migration (May-early June).

Conversely, individual captures of bushtit significantly increased throughout the MAPS banding season (appendix $\mathrm{C}$, fig. $\mathrm{C} 7 \mathrm{~B} ; r=0.56, P=0.09$ ), likely corresponding to the influx of juveniles and general increase in the movement of bushtit family groups post-fledging. Populations of Bewick's wren, wrentit, spotted towhee, and California towhee all had peaks in individual captures during late June and early July (Banding Periods 6 and 7; appendix C, fig. C7 C, D, F, G), corresponding to the fledgling period of the breeding cycle. 


\section{Productivity and Population Size}

Annual productivity varied by species, but was generally low in 2014 and higher in 2015 (appendix C, fig. C8). Bewick's wrens had the highest overall productivity, followed by bushtit and wrentit. orange-crowned warbler had the lowest overall productivity although it generally increased over 5 years. Productivity was a significant predictor of observed population size the next year for bushtit (appendix C, fig. C9 A), but not for any of the other resident species examined (appendix C, fig. C9 A-C).

\section{Survivorship and Population Size}

Adult survivorship also varied among years and species from 2011 through 2015 (appendix C, fig. C10). Adult survivorship was generally high from 2013 to 14 and low from 2014 to 15 (appendix C, fig. C10 A). Wrentits had the highest survivorship, followed by California towhees and orange-crowned warblers (appendix C, fig. C10 B). Adult survivorship was lowest for bushtits and spotted towhees. Adult survivorship was a significant predictor of population size for bushtits (appendix C, fig. C11 A), but not for the remaining five species (appendix C, fig. C11 A-C).

\section{Discussion}

Our monitoring results from 2011 to 2015 indicate that Point Loma provides stopover habitat during migration for 76 migratory species (54 captured and 22 observed only) and breeding habitat for 30 species (22 captured and 8 observed only). Additionally, Point Loma provides important stop-over habitat for migratory species of conservation concern, including northern harrier, calliope hummingbird, rufous hummingbird, Allen's hummingbird, olive-sided flycatcher, yellow warbler, Canada warbler, yellow-breasted chat, green-tailed towhee, black-chinned sparrow, sage sparrow, and Lawrence's goldfinch, and breeding habitat for species of conservation concern including peregrine falcon, whitethroated swift, Costa's hummingbird, wrentit, and California thrasher (U.S. Fish and Wildlife Service, 2008; Partners in Flight Science Committee, 2012; California Department of Fish and Wildlife, 2016a, 2016b, 2016c).

Point Loma also provides stopover, dispersal, and (or) breeding habitat for three State and Federally listed species, California gnatcatcher, least Bell's vireo, and willow flycatcher. Willow flycatchers were captured and banded all 5 years, for a total of 12 individuals, none of which were identified as the locally breeding southwestern willow flycatcher (subspecies extimus). This species was using Point Loma as migratory stop-over habitat because Point Loma lacks the riparian habitat required for breeding willow flycatchers. Least Bell's vireos were captured in 2013 (two individuals) and 2014 (two individuals). These four individuals were given unique color band combinations in addition to the standard metal federal band for visual identification on the breeding and wintering grounds. Similar to willow flycatchers, least Bell's vireos were using Point Loma as migratory stop-over or dispersal habitat because Point Loma lacks the riparian habitat required for this species to breed. We captured and banded two individual California gnatcatchers, one in 2013 and one in 2015. The individual captured in 2015 was given a unique color band combination in addition to the standard metal federal band so that local biologists could monitor the breeding and movements of this individual. This species was confirmed breeding on Point Loma in 2015. 
During spring migration across 5 years, the migrant capture rate increased from the beginning of April and peaked in late May, with a secondary peak in mid-April. Similarly, the number of species captured per day peaked in mid-April, with a secondary peak in early May. This is related to the timing of migration for different species (species composition varied during the two peaks). Of the most common species, white-crowned sparrows were captured most frequently in April and not captured at all in May. Rufous hummingbirds were most frequently captured in early to mid-April and in lower numbers through early May. The peaks in May were largely attributable to captures of warbling vireos, Wilson's warblers, and Pacific-slope flycatchers, all of which were captured in lower numbers during April but were most abundant in late April and May. The peaks in migrant captures also may be attributed to weather at the station, as they occurred on mornings of low or no cloud cover and the peninsula was likely more obvious to birds in flight.

The peaks in new species arrival have varied, but occurred by mid-April in all years. The first and median arrival dates of particular migrant species have varied from year-to-year over the 5 years of our monitoring but tended toward earlier arrival each year, or no difference in arrival date, with few species arriving later. The exceptions were black-chinned hummingbird and hermit warbler, which arrived later during the final years of our study than during the earlier years, according to median arrival dates. According to median arrival dates, rufous hummingbird arrived earlier in the last 2 years than in the first 3 years. Three of the most common spring migrant species, Pacific-slope flycatcher, warbling vireo, and Wilson's warbler, had variable median arrival dates each year, but all three had earliest median arrival dates in 2013. White-crowned sparrow median arrival dates tended to be earlier in later years, although the trend was not consistent across years. The changes in spring arrival dates are most likely attributed to weather, as the timing of spring migration is closely related to variations in weather (Lehikoinen and others, 2004; Van Buskirk and others, 2009). Median arrival dates better reflect the migratory behavior of entire populations, whereas first arrival dates are a record of a single individual (Lehikoinen and others, 2004, Van Buskirk and others, 2009; Newton, 2010), and are therefore strongly affected by individual variation within a population; thus, median arrival dates are better suited for analyzing year-to-year differences in arrival times and for analyzing responses to climate change.

The reflectivity in NEXRAD images during spring migration was positively associated with our bird capture rate at Point Loma across all 5 years of our study. This pattern was observed each year individually except for 2014, suggesting that there were some variables that introduced noise into the images, preventing a clear and easy interpretation. Such variables may include storm clouds or taxa of birds that we typically do not capture (for example, shorebirds, waterfowl) that create false or misleading echoes or block true echoes of bird over-flight. Therefore, if applied cautiously, NEXRAD imagery in San Diego is useful in determining when spring migrants arrive at Point Loma, and also the relative abundance of these migrants. Under clear weather conditions, we should be able to roughly predict days when migrant birds are abundant by examining NEXRAD images during the first few hours after sunset.

The capture rate of one migratory species (white-crowned sparrow) decreased, and the capture rate of one migratory species (Pacific-slope flycatcher) increased during the 5 years of our study. Potential causes of these trends in capture rates are varied, and could include increasingly early migration of white-crowned sparrows (individuals migrating before our banding effort began at the beginning of April), weather-related shifts in migratory corridors (weather may drive migrants inland some years and not in others), weather-related pauses and pushes in migration (nets might not have been open during migratory pulses; that is, weekends), and actual decreases or increases in population size. Some of these factors may be supported by data collected at other banding stations (MAPS and Monitoring Overwinter Survival program, also coordinated by the Institute for Bird Populations). Our examination of radar data suggests that weather can affect migration; however radar data cannot 
distinguish between species so has limited applicability for studies of population trends of individual species. These factors may be best examined by establishing a wide array of banding stations covering a longitudinal range to encompass more possible migratory corridors, beginning banding operations earlier in the year, and operating these stations on days when radar data from the previous night indicate a migratory pulse.

Using capture rate (captures per 100 net-hours) as an index of abundance, all seven of the most common neotropical migrant species captured during the breeding season decreased in abundance from 2011 to 2012, and all but Anna's hummingbird increased in abundance from 2014 to 2015. These trends were influenced by the number of juveniles produced that year (the ratio of juveniles to adults), excluding Anna's hummingbird, for which productivity was not calculated. The ratio of juveniles to adults was higher in 2011 than in 2012 for all species except bushtit, and was lower in 2014 than 2015 for all six species.

However, breeding productivity alone did not translate to abundance in the subsequent year, except for bushtits. Adult survivorship from one year to the next was also only a predictor of abundance for bushtits. Examination of year-to-year abundance, productivity, and adult survivorship for the five other common species does not clarify causes for population trends in this study site and period. However, adult survivorship from one year to the next at our study site was higher than range-wide adult survivorship calculated for all of these species except spotted towhee between 1992 and 2006 (39 percent to 63 percent; DeSante and others, 2015). Average productivity at our study site was also higher than range-wide productivity calculations for all species except orange-crowned warbler between 1992 and 2006 (0.37-1.17; DeSante and others, 2015). Only bushtit and orange-crowned warbler populations significantly decreased at our study site during the 5 years of our study. Range-wide population statistics have not yet been released for more recent years, but based on our data relative to DeSante and others (2015), populations at our study site appear to be doing better than the species range-wide except for orange-crowned warbler. Orange-crowned warbler decreased in our study area during the 5 years of our study and also had lower productivity at our study site than the range-wide average (DeSante and others, 2015).

Other potential drivers of population abundance were likely juvenile survivorship, immigration rates, and emigration rates. Our small sample size of recaptured juveniles precluded analysis of juvenile survivorship and suggests that not many juveniles survived into their second year, the opposite of what we would expect if juvenile survivorship rates were sufficient to significantly increase or maintain population size. Additionally, survival analysis does not capture immigration and emigration rates because it assumes a closed system: that loss of an individual was a result of death, rather than the movement of an individual out of the study area. Changes in population size are likely affected by movements of birds into and out of the study area, movements that we did not have the capacity to measure. Similarly, an assumption of MAPS banding is that we captured all or a consistent proportion of individuals of a species, with new adult captures resulting from the proportion not captured in previous years rather than immigrants from outside the study area. These potential influences could be elucidated by sampling populations outside of the MAPS study area but in reasonable range for moving individuals. 


\section{Management Implications}

Based on capture rates for the 5 years of our study, bushtit, Bewick's wren, wrentit, orangecrowned warbler, spotted towhee, and California towhee may have capture rates sufficient for long-term monitoring of population size and composition, survivorship, productivity, and recruitment using the MAPS mist-netting and banding protocol. These are also among the most abundant species captured at four additional MAPS stations operated recently by U.S. Geological Survey (USGS) on Department of Defense lands in San Diego County, making regional comparisons feasible (Lynn and others, 2014). Anna's hummingbird also may have adequate numbers, but they are difficult to band and recapture and would require a concentrated effort beyond the scope of a MAPS station. For effective monitoring using the MAPS protocol, a MAPS station should be operated each year for a minimum of 5 years (DeSante and others, 2011).

California gnatcatchers were confirmed breeding on Point Loma in 2015, the first time this species has been detected breeding there since before 1997 (Mock, 2004). Persistence of this species on Point Loma requires preservation of their coastal sage scrub habitat, suppression of fires, and removal of exotic, flammable vegetation where present. Periodic surveys for California gnatcatcher would contribute to knowledge of the regional distribution of this species.

Bushtit and orange-crowned warbler populations experienced declining trends during the 5 years of our study. Both of these species are relatively common regionally, and may have been affected by the on-going drought in San Diego County. It is unclear why other species were less affected by drought than these two resident species. Maintaining the current natural habitat components on Point Loma while controlling exotic and invasive plants and animals and limiting damaging wildfires are all management actions that likely would aid in sustaining healthy bird populations, both for year-roundresidents and for those that use Point Loma only during part of their annual cycle, and allow these populations to rebound when climate conditions improve.

The 5-year MAPS/migration dataset is comprehensive and provides a solid foundation for future comparisons, but alternatives are available for continued monitoring depending on goals and available resources. Periodic point counts or area searches could be useful for monitoring trends in the most abundant species; however, if data on productivity and survival are desired, MAPS is the better option. To focus on breeding birds, migration monitoring would not be necessary; conversely, if the focus is on arrival dates and use of the site by migrating birds, breeding season MAPS may not be needed. 


\section{References Cited}

Able, K.P., 1970, A radar study of the altitude of nocturnal passerine migration: Bird-Banding, v. 41, p. 282-290.

Baltosser, W.H., and Russell, S.M., 2000, Black-chinned Hummingbird (Archilochus alexandri), in Poole, A., ed., The Birds of North America Online: Ithaca, New York, Cornell Lab of Ornithology, accessed February 15, 2017, at https://birdsna.org/Species-Account/bna/species/bkchum/breeding.

California Department of Fish and Wildlife, 2016a, Fully protected animals: California Department of Fish and Wildlife, accessed August 25, 2016, at http://www.dfg.ca.gov/wildlife/nongame/t_e_spp/fully_pro.html\#Birds.

California Department of Fish and Wildlife, 2016b, Special animals list, Natural Diversity Database, Periodic publication, April 2016, 51 p., accessed August 25, 2016, at https://nrm.dfg.ca.gov/FileHandler.ashx?DocumentID=109406\&inline.

California Department of Fish and Wildlife, 2016c, State and Federally listed endangered and threatened animals of California: California Department of Fish and Wildlife, accessed August 25, 2016, at https://nrm.dfg.ca.gov/FileHandler.ashx?DocumentID=109405\&inline.

DeSante, D.F., Burton, K.M., Velez, P., Froehlich, D., and Kaschube, D., 2011, MAPS manualInstructions for the establishment and operation of constant-effort bird-banding stations as part of the Monitoring Avian Productivity and Survivorship (MAPS) Program: Point Reyes Station, California, Institute for Bird Populations.

DeSante, D.F., Kaschube, D.R., and Saracco, J.F., 2015, Vital rates of North American landbirds: The Institute of Bird Populations, accessed August 25, 2016, at

www.VitalRatesOfNorthAmericanLandbirds.org.

Diehl, R.H., and Larkin, R.P., 2004, Introduction to the WSR-88D (NEXRAD) for ornithological research: U.S. Forest Service General Technical Report PSW-GTR-191, 13 p.

Lack, D., and Varley, G.C., 1945, Detection of birds by radar: Nature, v. 156, p. 446.

Lehikoinen, E., Sparks, T.H., and Zalakevicius, M., 2004, Arrival and departure dates: Advances in Ecological Research, v. 35, p. 1-31.

Lincoln, F.C., Peterson, S.R., and Zimmerman, J.L., 1998, Migration of birds: Washington, D.C., U.S. Fish and Wildlife Service, Circular 16, 113 p.

Lynn, S., Madden, M., Houston, A., and Kus, B.E., 2014, Monitoring avian productivity and survivorship (MAPS) 5-year summary, Naval Outlying Landing Field, Imperial Beach, southwestern San Diego County, California, 2009-13: U.S. Geological Survey Open-File Report 2015-1035, 58 p., https://pubs.er.usgs.gov/publication/ofr20151035.

Mock, P.J., 2004, California gnatcatcher Polioptila californica, in Unitt, P., San Diego County Bird Atlas: San Diego Natural History Museum, p. 430-433.

National Oceanic and Atmospheric Administration, 2016, NOAA's weather and climate toolkit: National Oceanic and Atompsheric Adminstration website, accessed August 25, 2016, at https://www.ncdc.noaa.gov/wct/.

Newton, I., 2010, The migration ecology of birds: Great Britain, Academic Press, 984 p..

O’Neal, B.J., Stafford, J.D., and Larkin, R.P., 2010, Waterfowl on weather radar-Applying groundtruth to classify and quantify bird movements: Journal of Field Ornithology, v. 81, p. 71-82.

Partners in Flight Science Committee, 2012, Species assessment database, ver. 2012: Partners in Flight Committee, accessed August 25, 2016, at http://rmbo.org/pifassessment.

Pyle, P., 1997, Identification guide to North American Birds, part 1-Columbidae to Ploceidae: Bolinas, California: Slate Creek Press, 732 p. 
Randall, L.A., Diehl, R.H., Wilson, B.C., Barrow, W.C., Jr., and Jeske, C.W., 2011, Potential use of weather radar to study movements of wintering waterfowl: The Journal of Wildlife Management, $\mathrm{v}$. 75 , p. $1,324-1,329$.

Schukman, J.M., and Wolf, B.O., 1998, Say's phoebe (Sayornis saya), in Poole, A., ed., The Birds of North America Online: Cornell Lab of Ornithology, accessed February 15, 2017 at https://birdsna.org/Species-Account/bna/species/saypho/breeding.

Skagen, S.K., Melcher, C.P., and Hazelwood, R., 2004, Migration stopover ecology of Western avian populations-A southwestern migration workshop: U.S. Geological Survey Open-File Report 20041452, 28 p. [Also available at https://pubs.er.usgs.gov/publication/ofr20041452.]

U.S. Fish and Wildlife Service, 2008, Birds of conservation concern 2008: Arlington, Virginia, U.S. Fish and Wildlife Service, Division of Migratory Bird Management, 85 p., accessed August 25, 2016 at https://www.fws.gov/migratorybirds/pdf/management/BCC2008.pdf.

U.S. Fish and Wildlife Service, 2011, Neotropical Migratory Bird Conservation Act: U.S. fish and Wildlife Service, accessed August 25, 2016 at http://www.fws.gov/birdhabitat/Grants/NMBCA/BirdList.shtm.

Van Buskirk, J., Mulvihill, R.S., and Lieberman, R.C., 2009, Variable shifts in spring and autumn migration phenology in North American songbirds associated with climate change: Global Change Biology, v. 15, p. 760-771. 


\section{Appendix A. Alpha Codes, Common Names, and Scientific Names for Species Captured and Observed, Point Loma, San Diego, California, 2011-15.}

Table A1. Species captured at the Point Loma banding station, San Diego, California, 2011-15.

[Species with alpha codes in italics are non-breeding migrants or transients. Special status: CC, Partners in Flight Species of Conservation Concern (U.S. Fish and Wildlife Service, 2008; Partners in Flight Science Committee, 2012; California Department of Fish and Wildlife 2016a-c); FC, Federal Species of conservation concern; FE, Federally listed as endangered; FT, Federally listed as threatened; SC, California State species of concern; SE, California State listed as endangered]

\begin{tabular}{|c|c|c|c|}
\hline Alpha code & Common name & Scientific name & Special status \\
\hline SSHA & Sharp-shinned hawk & Accipiter striatus & \\
\hline$A M K E^{1}$ & American kestrel & Falco sparvarius & \\
\hline CAQU & California quail & Callipepla californica & \\
\hline MODO1 & Mourning dove & Zenaida macroura & \\
\hline $\mathrm{BCHU}^{1}$ & Black-chinned hummingbird & Archilochus alexandri & \\
\hline ANHU1 & Anna's hummingbird & Calypte anna & \\
\hline $\mathrm{COHU} 1$ & Costa's hummingbird & Calypte costae & $\mathrm{CC}, \mathrm{FC}$ \\
\hline $\mathrm{CAHU}^{1}$ & Calliope hummingbird & Selasphorus calliope & $\mathrm{CC}, \mathrm{FC}$ \\
\hline RUHU1 & Rufous hummingbird & Selasphorus rufus & $\mathrm{CC}, \mathrm{FC}$ \\
\hline$A L H U^{1}$ & Allen's hummingbird & Selasphorus sasin & $\mathrm{CC}, \mathrm{FC}$ \\
\hline USHU1 & $\begin{array}{l}\text { Unidentified Selasphorus hummingbird } \\
\text { species }\end{array}$ & Selasphorus spp. & \\
\hline UNHU1 & Unidentified hummingbird species & --- & \\
\hline DOWO & Downy woodpecker & Picoides pubescens & \\
\hline WEWP1 & Western wood-pewee & Contopus sordidulus & \\
\hline WIFL ${ }^{1}$ & Willow flycatcher & Empidonax traillii & $\mathrm{CC}, \mathrm{FC}, \mathrm{SE}$ \\
\hline$L E F L^{1}$ & Least flycatcher & Empidonax minimus & \\
\hline$H A F L^{1}$ & Hammond's flycatcher & Empidonax hammondii & \\
\hline$G R F L^{1}$ & Gray flycatcher & Empidonax wrightii & \\
\hline DUFL ${ }^{1}$ & Dusky flycatcher & Empidonax oberholseri & \\
\hline COFL 1 & Cordilleran flycatcher & Empidonax occidentalis & \\
\hline$P S F L^{1}$ & Pacific-slope flycatcher & Empidonax difficilis & \\
\hline BLPH & Black phoebe & Sayornis nigricans & \\
\hline$S A P H^{1}$ & Say's phoebe & Sayornis saya & \\
\hline ATFL 1 & Ash-throated flycatcher & Myiarchus cinerascens & \\
\hline $\mathrm{CAKI}^{1}$ & Cassin's kingbird & Tyrannus vociferans & \\
\hline WEKI 1 & Western kingbird & Tyrannus verticalis & \\
\hline$L B V I^{1}$ & Least Bell's vireo & Vireo bellii pusillus & CC, FE. SE \\
\hline$C A V I^{1}$ & Cassin's vireo & Vireo cassinii & \\
\hline WAVI 1 & Warbling vireo & Vireo gilvus & \\
\hline$R E V I^{1}$ & Red-eyed vireo & Vireo olivaceus & \\
\hline WESJ & Western scrub-jay & Aphelocoma californica & \\
\hline
\end{tabular}




\begin{tabular}{|c|c|c|c|}
\hline Alpha code & Common name & Scientific name & Special status \\
\hline BARS 1 & Barn swallow & Hirundo rustica & \\
\hline $\mathrm{BUSH}$ & Bushtit & Psaltriparus minimus & \\
\hline BEWR & Bewick's wren & Thryomanes bewickii & \\
\hline HOWR ${ }^{1}$ & House wren & Troglodytes aedon & \\
\hline$R C K I^{1}$ & Ruby-crowned kinglet & Regulus calendula & \\
\hline$B G G N^{1}$ & Blue-gray gnatcatcher & Polioptila caerulea & \\
\hline CAGN & California gnatcatcher & Polioptila californica californica & $\mathrm{CC}, \mathrm{FT}$ \\
\hline SWTH & Swainson's thrush & Catharus ustulatus & \\
\hline$H E T H^{1}$ & Hermit thrush & Catharus guttatus & \\
\hline WREN & Wrentit & Chamaea fasciata & CC \\
\hline$G R C A^{1}$ & Gray catbird & Dumetella carolinensis & \\
\hline NOMO & Northern mockingbird & Mimus polyglottos & \\
\hline CATH & California thrasher & Toxostoma redivivum & $\mathrm{CC}$ \\
\hline PHAl1 & Phainopepla & Phainopepla nitens & \\
\hline OCWA1 & Orange-crowned warbler & Oreothlypis celata & \\
\hline$N A W A^{1}$ & Nashville warbler & Oreothlypis ruficapilla & \\
\hline YEWA1 & Yellow warbler & Setophaga petechia & SC \\
\hline$A \cup W A^{1}$ & Audubon's warbler & Setophaga coronata auduboni & \\
\hline$B T Y W^{1}$ & Black-throated Gray warbler & Setophaga nigrescens & \\
\hline TOWA1 & Townsend's warbler & Setophaga townsendi & \\
\hline$T H W H^{1}$ & Townsend's $x$ hermit warbler Hybrid & Setophaga townsendi x occidentalis & \\
\hline HEWA & Hermit warbler & Setophaga occidentalis & \\
\hline$M G W A^{1}$ & MacGillivray's warbler & Geothlypis tolmiei & \\
\hline COYE $^{1}$ & Common Yellowthroat & Geothlypis trichas & \\
\hline WIWA & Wilson's warbler & Cardellina pusilla & \\
\hline$C A W A^{1}$ & Canada warbler & Wilsonia canadensis & $\mathrm{CC}, \mathrm{FC}$ \\
\hline $\mathrm{YBCH}^{1}$ & Yellow-breasted chat & Icteria virens & SC \\
\hline WETA ${ }^{1}$ & Western tanager & Piranga ludoviciana & \\
\hline GTTO $^{1}$ & Green-tailed towhee & Pipilo chlorurus & FC \\
\hline SPTO $^{1}$ & Spotted towhee & Pipilo maculatus & \\
\hline CALT & California towhee & Melozone crissalis & \\
\hline $\mathrm{CHSP}^{1}$ & Chipping sparrow & Spizella passerina & \\
\hline$B C S P^{1}$ & Black-chinned sparrow & Spizella atrogularis & $\mathrm{FC}$ \\
\hline SAGS1 & Sage sparrow & Amphispiza belli & FC \\
\hline SAVS1 & Savannah sparrow & Passerculus sandwichensis & \\
\hline FOSP 1 & Fox sparrow & Passerella iliaca & \\
\hline SOSP1 & Song sparrow & Melospiza melodia & \\
\hline$L I S P^{1}$ & Lincoln's sparrow & Melospiza lincolnii & \\
\hline WTSP2 & White-throated sparrow & Zonotrichia albicollis & \\
\hline WCSP ${ }^{1}$ & White-crowned sparrow & Zonotrichia leucophrys & \\
\hline GCSP1 & Golden-crowned sparrow & Zonotrichia atricapilla & \\
\hline
\end{tabular}




\begin{tabular}{|c|c|c|c|}
\hline Alpha code & Common name & Scientific name & Special status \\
\hline ORJU1 & Oregon junco & Junco hyemalis & \\
\hline$B H G R^{1}$ & Black-headed grosbeak & Pheucticus melanocephalus & \\
\hline$B L G R^{1}$ & Blue grosbeak & Passerina caerulea & \\
\hline $\mathrm{LAZB}^{1}$ & Lazuli bunting & Passerina amoena & \\
\hline$I^{N} B U^{1}$ & Indigo bunting & Passerina cyanea & \\
\hline OROR ${ }^{1}$ & Orchard oriole & Icterus spurius & \\
\hline $\mathrm{HOOR}^{1}$ & Hooded oriole & Icterus cucullatus & \\
\hline$B \cup O R^{1}$ & Bullock's oriole & Icterus bullockii & \\
\hline $\mathrm{HOFI}$ & House finch & Haemorhous mexicanus & \\
\hline LEGO$^{1}$ & Lesser goldfinch & Spinus psaltria & \\
\hline$A M G O^{1}$ & American goldfinch & Spinus tristis & \\
\hline
\end{tabular}

${ }^{1}$ Species defined as neotropical migratory bird under the Neotropical Migratory Bird Conservation Act of 2000 (U.S. Fish and Wildlife Service, 2013).

${ }^{2}$ Migratory species, but migrates only within North America and thus not considered a neotropical migratory species. 
Table A2. Species observed but not captured at the Point Loma banding station, San Deigo, California, 2011-15.

[Species with alpha codes in italics are non-breeding migrants or transients. Special status: CC, partners in Flight Species of Conservation Concern (U.S. Fish and Wildlife Service 2008, Partners in Flight Science Committee 2012, California Department of Fish and Wildlife 2016a, b, c); FC, Federal species of conservation concern; FE, Federally listed as endangered; FT, Federally listed as threatened; SC, California State species of concern; SE, California State listed as endangered]

\begin{tabular}{|c|c|c|c|}
\hline Alpha code & Common name & Scientific name & Special status \\
\hline TUVU1 & Turkey vulture & Cathartes aura & \\
\hline OSPR 1 & Osprey & Pandion haliaetus & \\
\hline WTKI & White-tailed kite & Elanus leucurus & \\
\hline $\mathrm{NOH} A^{1}$ & Northern harrier & Circus cyaneus & SC \\
\hline $\mathrm{COHA}^{1}$ & Cooper's hawk & Accipiter cooperii & \\
\hline $\mathrm{RTHA}^{1}$ & Red-tailed hawk & Buteo jamaicensis & \\
\hline PEFA $^{1}$ & Peregrine falcon & Falco peregrinus & FC \\
\hline ROPI & Rock pigeon & Columba livia & \\
\hline EUCD & Eurasian collared-dove & Streptopelia decaocto & \\
\hline BANO & Barn owl & Tyto alba & \\
\hline GHOW & Great Horned owl & Bubo virginianus & \\
\hline LENI $^{1}$ & Lesser nighthawk & Chordeiles acutipennis & \\
\hline WTSW ${ }^{1}$ & White-throated swift & Aeronautes saxatalis & $\mathrm{CC}$ \\
\hline OSFL ${ }^{1}$ & Olive-sided flycatcher & Contopus cooperi & $\mathrm{CC}, \mathrm{FC}, \mathrm{SC}$ \\
\hline$A C F L^{1}$ & Acadian flycatcher & Empidonax virescens & \\
\hline AMCR & American crow & Corvus brachyrhynchos & \\
\hline CORA & Common raven & Corvus corax & \\
\hline VGSW'1 & Violet-green swallow & Tachycineta thalassina & \\
\hline$N R W S^{1}$ & Northern Rough-winged swallow & Stelgidopteryx serripennis & \\
\hline $\mathrm{CLSW}^{1}$ & Cliff swallow & Petrochelidon pyrrhonota & \\
\hline RBNU & Red-breasted nuthatch & Sitta canadensis & \\
\hline WBNU & White-breasted nuthatch & Sitta carolinensis & \\
\hline ROWR & Rock wren & Salpinctes obsoletus & \\
\hline WEBL ${ }^{1}$ & Western bluebird & Sialia mexicana & \\
\hline EUST & European starling & Sturnus vulgaris & \\
\hline CEDW ${ }^{1}$ & Cedar waxwing & Bombycilla cedrorum & \\
\hline$R C S P$ & Rufous-crowned sparrow & Aimophila ruficeps & \\
\hline$B R B L^{1}$ & Brewer's blackbird & Euphagus cyanocephalus & \\
\hline GTGR & Great-tailed grackle & Quiscalus mexicanus & \\
\hline$\angle A G O^{1}$ & Lawrence's goldfinch & Spinus lawrencei & $\mathrm{CC}, \mathrm{FC}$ \\
\hline
\end{tabular}

${ }^{1}$ Species defined as Neotropical migratory bird under the Neotropical Migratory Bird Conservation Act of 2000 (U.S. Fish and Wildlife Service, 2013). 


\section{Appendix B. Bird Species Captured and Banded during Spring Migration 2011-15, Point Loma, California.}

Table B1. Number of migrant birds captured, banded, and recaptured during spring migration, Point Loma banding station, San Diego, California 2011-15.

[See appendix A for bird species codes. Total captures includes multiple captures of some individuals. Recaptures originally banded during previous USGS banding activities at Point Loma, 2010-2014. No birds banded in previous years were recaptured during spring migration in 2011, 2012, 2013, or 2015]

\begin{tabular}{|c|c|c|c|c|c|c|c|c|c|c|c|c|c|c|c|c|c|c|c|c|c|}
\hline \multirow{3}{*}{$\begin{array}{l}\text { Species } \\
\text { code }\end{array}$} & \multicolumn{6}{|c|}{ Total captures } & \multicolumn{6}{|c|}{ Number of individuals captured } & \multicolumn{6}{|c|}{ New individuals banded } & \multicolumn{3}{|c|}{$\begin{array}{c}\text { Recaptured } \\
\text { individuals, } 2014\end{array}$} \\
\hline & \multicolumn{5}{|c|}{ Year } & \multirow[b]{2}{*}{ Total } & \multicolumn{5}{|c|}{ Year } & \multirow[b]{2}{*}{ Total } & \multicolumn{5}{|c|}{ Year } & \multirow[b]{2}{*}{ Total } & \multicolumn{2}{|c|}{$\begin{array}{c}\text { Year } \\
\text { originally } \\
\text { banded }\end{array}$} & \multirow[b]{2}{*}{ Tota } \\
\hline & 2011 & 2012 & 2013 & 2014 & 2015 & & 2011 & 2012 & 2013 & 2014 & 2015 & & 2011 & 2012 & 2013 & 2014 & 2015 & & 2012 & 2013 & \\
\hline SSHA & 0 & 0 & 1 & 0 & 0 & 1 & 0 & 0 & 1 & 0 & 0 & 1 & 0 & 0 & 0 & 0 & 0 & 0 & 0 & 0 & 0 \\
\hline AMKE & 0 & 0 & 0 & 0 & 1 & 1 & 0 & 0 & 0 & 0 & 1 & 1 & 0 & 0 & 0 & 0 & 0 & 0 & 0 & 0 & 0 \\
\hline $\mathrm{BCHU}$ & 0 & 3 & 4 & 1 & 6 & 14 & 0 & 3 & 4 & 1 & 6 & 14 & 0 & 0 & 0 & 0 & 0 & 0 & 0 & 0 & 0 \\
\hline DOWO & 0 & 0 & 0 & 2 & 0 & 2 & 0 & 0 & 0 & 2 & 0 & 2 & 0 & 0 & 0 & 2 & 0 & 2 & 0 & 0 & 0 \\
\hline WEWP & 2 & 5 & 2 & 1 & 6 & 16 & 2 & 5 & 2 & 1 & 6 & 16 & 2 & 5 & 2 & 1 & 6 & 16 & 0 & 0 & 0 \\
\hline WIFL & 2 & 1 & 0 & 1 & 3 & 7 & 2 & 1 & 0 & 1 & 3 & 7 & 2 & 1 & 0 & 1 & 3 & 7 & 0 & 0 & 0 \\
\hline LEFL & 0 & 1 & 0 & 0 & 0 & 1 & 0 & 1 & 0 & 0 & 0 & 1 & 0 & 1 & 0 & 0 & 0 & 1 & 0 & 0 & 0 \\
\hline HAFL & 7 & 4 & 5 & 8 & 15 & 39 & 7 & 4 & 5 & 8 & 15 & 39 & 7 & 4 & 5 & 8 & 15 & 39 & 0 & 0 & 0 \\
\hline WEKI & 0 & 0 & 1 & 1 & 0 & 2 & 0 & 0 & 1 & 1 & 0 & 2 & 0 & 0 & 1 & 1 & 0 & 2 & 0 & 0 & 0 \\
\hline LBVI & 0 & 0 & 0 & 1 & 0 & 1 & 0 & 0 & 0 & 1 & 0 & 1 & 0 & 0 & 0 & 1 & 0 & 1 & 0 & 0 & 0 \\
\hline CAVI & 0 & 1 & 1 & 0 & 1 & 3 & 0 & 1 & 1 & 0 & 1 & 3 & 0 & 1 & 1 & 0 & 1 & 3 & 0 & 0 & 0 \\
\hline WAVI & 47 & 21 & 16 & 48 & 47 & 179 & 46 & 21 & 16 & 48 & 46 & 177 & 46 & 21 & 16 & 48 & 46 & 177 & 0 & 0 & 0 \\
\hline HOWR & 1 & 1 & 2 & 1 & 0 & 5 & 1 & 1 & 1 & 1 & 0 & 4 & 1 & 1 & 1 & 0 & 0 & 3 & 0 & 1 & 1 \\
\hline RCKI & 2 & 0 & 30 & 19 & 6 & 57 & 2 & 0 & 27 & 16 & 5 & 50 & 2 & 0 & 27 & 15 & 5 & 49 & 0 & 1 & 1 \\
\hline BGGN & 1 & 0 & 0 & 1 & 0 & 2 & 1 & 0 & 0 & 1 & 0 & 2 & 1 & 0 & 0 & 1 & 0 & 2 & 0 & 0 & 0 \\
\hline
\end{tabular}




\begin{tabular}{|c|c|c|c|c|c|c|c|c|c|c|c|c|c|c|c|c|c|c|c|c|c|}
\hline \multirow{3}{*}{$\begin{array}{l}\text { Species } \\
\text { code }\end{array}$} & \multicolumn{6}{|c|}{ Total captures } & \multicolumn{6}{|c|}{ Number of individuals captured } & \multicolumn{6}{|c|}{ New individuals banded } & \multicolumn{3}{|c|}{$\begin{array}{c}\text { Recaptured } \\
\text { individuals, } 2014\end{array}$} \\
\hline & \multicolumn{5}{|c|}{ Year } & \multirow[b]{2}{*}{ Total } & \multicolumn{5}{|c|}{ Year } & \multirow[b]{2}{*}{ Total } & \multicolumn{5}{|c|}{ Year } & \multirow[b]{2}{*}{ Total } & \multicolumn{2}{|c|}{$\begin{array}{c}\text { Year } \\
\text { originally } \\
\text { banded }\end{array}$} & \multirow[b]{2}{*}{ Total } \\
\hline & 2011 & 2012 & 2013 & 2014 & 2015 & & 2011 & 2012 & 2013 & 2014 & 2015 & & 2011 & 2012 & 2013 & 2014 & 2015 & & 2012 & 2013 & \\
\hline SWTH & 10 & 4 & 2 & 8 & 2 & 26 & 10 & 4 & 2 & 8 & 2 & 26 & 10 & 4 & 2 & 8 & 2 & 26 & 0 & 0 & 0 \\
\hline HETH & 8 & 21 & 10 & 15 & 11 & 65 & 8 & 15 & 10 & 12 & 10 & 55 & 8 & 15 & 10 & 11 & 10 & 54 & 1 & 0 & 1 \\
\hline GRCA & 0 & 0 & 0 & 0 & 1 & 1 & 0 & 0 & 0 & 0 & 1 & 1 & 0 & 0 & 0 & 0 & 1 & 1 & 0 & 0 & 0 \\
\hline PHAI & 1 & 0 & 0 & 0 & 0 & 1 & 1 & 0 & 0 & 0 & 0 & 1 & 1 & 0 & 0 & 0 & 0 & 1 & 0 & 0 & 0 \\
\hline NAWA & 24 & 7 & 8 & 5 & 8 & 52 & 22 & 7 & 8 & 5 & 8 & 50 & 22 & 7 & 8 & 5 & 8 & 50 & 0 & 0 & 0 \\
\hline YEWA & 3 & 1 & 0 & 5 & 5 & 14 & 3 & 1 & 0 & 5 & 5 & 14 & 3 & 1 & 0 & 5 & 5 & 14 & 0 & 0 & 0 \\
\hline AUWA & 2 & 4 & 5 & 2 & 0 & 13 & 2 & 4 & 5 & 2 & 0 & 13 & 2 & 4 & 5 & 2 & 0 & 13 & 0 & 0 & 0 \\
\hline BTYW & 12 & 3 & 5 & 5 & 8 & 33 & 12 & 3 & 5 & 5 & 8 & 33 & 12 & 3 & 5 & 5 & 8 & 33 & 0 & 0 & 0 \\
\hline TOWA & 30 & 9 & 8 & 26 & 14 & 87 & 30 & 9 & 8 & 25 & 14 & 86 & 30 & 9 & 8 & 24 & 14 & 85 & 0 & 0 & 0 \\
\hline THWH & 3 & 0 & 0 & 2 & 0 & 5 & 3 & 0 & 0 & 2 & 0 & 5 & 3 & 0 & 0 & 2 & 0 & 5 & 0 & 0 & 0 \\
\hline HEWA & 12 & 9 & 4 & 5 & 2 & 32 & 12 & 9 & 4 & 5 & 2 & 32 & 12 & 9 & 4 & 4 & 2 & 31 & 0 & 0 & 0 \\
\hline MGWA & 2 & 2 & 7 & 9 & 1 & 21 & 2 & 2 & 7 & 9 & 1 & 21 & 2 & 2 & 7 & 9 & 1 & 21 & 0 & 0 & 0 \\
\hline COYE & 0 & 2 & 3 & 4 & 1 & 10 & 0 & 2 & 3 & 4 & 1 & 10 & 0 & 2 & 3 & 4 & 1 & 10 & 0 & 0 & 0 \\
\hline WIWA & 90 & 77 & 53 & 68 & 82 & 370 & 89 & 76 & 51 & 66 & 82 & 364 & 89 & 75 & 50 & 63 & 81 & 358 & 0 & 0 & 0 \\
\hline CAWA & 0 & 0 & 0 & 0 & 1 & 1 & 0 & 0 & 0 & 0 & 1 & 1 & 0 & 0 & 0 & 0 & 1 & 1 & 0 & 0 & 0 \\
\hline $\mathrm{YBCH}$ & 1 & 0 & 0 & 0 & 0 & 1 & 1 & 0 & 0 & 0 & 0 & 1 & 1 & 0 & 0 & 0 & 0 & 1 & 0 & 0 & 0 \\
\hline WETA & 4 & 2 & 5 & 4 & 3 & 18 & 4 & 2 & 5 & 4 & 3 & 18 & 4 & 2 & 5 & 4 & 3 & 18 & 0 & 0 & 0 \\
\hline GTTO & 1 & 0 & 0 & 0 & 0 & 1 & 1 & 0 & 0 & 0 & 0 & 1 & 1 & 0 & 0 & 0 & 0 & 1 & 0 & 0 & 0 \\
\hline CHSP & 5 & 1 & 1 & 2 & 1 & 10 & 5 & 1 & 1 & 2 & 1 & 10 & 3 & 1 & 1 & 2 & 1 & 8 & 0 & 0 & 0 \\
\hline BCSP & 2 & 0 & 0 & 0 & 0 & 2 & 2 & 0 & 0 & 0 & 0 & 2 & 2 & 0 & 0 & 0 & 0 & 2 & 0 & 0 & 0 \\
\hline SAGS & 0 & 0 & 0 & 1 & 0 & 1 & 0 & 0 & 0 & 1 & 0 & 1 & 0 & 0 & 0 & 1 & 0 & 1 & 0 & 0 & 0 \\
\hline SAVS & 0 & 1 & 1 & 0 & 0 & 2 & 0 & 1 & 1 & 0 & 0 & 2 & 0 & 0 & 1 & 0 & 0 & 1 & 0 & 0 & 0 \\
\hline FOSP & 0 & 1 & 2 & 5 & 0 & 8 & 0 & 1 & 2 & 5 & 0 & 8 & 0 & 1 & 2 & 5 & 0 & 8 & 0 & 0 & 0 \\
\hline LISP & 4 & 8 & 12 & 1 & 4 & 29 & 4 & 7 & 12 & 1 & 4 & 28 & 3 & 7 & 12 & 1 & 4 & 27 & 0 & 0 & 0 \\
\hline WTSP & 1 & 0 & 0 & 0 & 0 & 1 & 1 & 0 & 0 & 0 & 0 & 1 & 1 & 0 & 0 & 0 & 0 & 1 & 0 & 0 & 0 \\
\hline WCSP & 37 & 38 & 41 & 20 & 12 & 148 & 36 & 38 & 41 & 20 & 11 & 146 & 34 & 37 & 41 & 20 & 11 & 143 & 0 & 0 & 0 \\
\hline GCSP & 11 & 3 & 7 & 5 & 4 & 30 & 10 & 3 & 6 & 4 & 4 & 27 & 10 & 3 & 6 & 4 & 4 & 27 & 0 & 0 & 0 \\
\hline ORJU & 0 & 0 & 0 & 1 & 0 & 1 & 0 & 0 & 0 & 1 & 0 & 1 & 0 & 0 & 0 & 1 & 0 & 1 & 0 & 0 & 0 \\
\hline BHGR & 20 & 4 & 9 & 3 & 5 & 41 & 20 & 4 & 9 & 3 & 5 & 41 & 20 & 4 & 9 & 3 & 5 & 41 & 0 & 0 & 0 \\
\hline BLGR & 0 & 1 & 0 & 0 & 0 & 1 & 0 & 1 & 0 & 0 & 0 & 1 & 0 & 1 & 0 & 0 & 0 & 1 & 0 & 0 & 0 \\
\hline
\end{tabular}




\begin{tabular}{|c|c|c|c|c|c|c|c|c|c|c|c|c|c|c|c|c|c|c|c|c|c|}
\hline \multirow{3}{*}{$\begin{array}{l}\text { Species } \\
\text { code }\end{array}$} & \multicolumn{6}{|c|}{ Total captures } & \multicolumn{6}{|c|}{ Number of individuals captured } & \multicolumn{6}{|c|}{ New individuals banded } & \multicolumn{3}{|c|}{$\begin{array}{c}\text { Recaptured } \\
\text { individuals, } 2014\end{array}$} \\
\hline & \multicolumn{5}{|c|}{ Year } & \multirow[b]{2}{*}{ Total } & \multicolumn{5}{|c|}{ Year } & \multirow[b]{2}{*}{ Total } & \multicolumn{5}{|c|}{ Year } & \multirow[b]{2}{*}{ Total } & \multicolumn{2}{|c|}{$\begin{array}{c}\text { Year } \\
\text { originally } \\
\text { banded }\end{array}$} & \multirow[b]{2}{*}{ Tota } \\
\hline & 2011 & 2012 & 2013 & 2014 & 2015 & & 2011 & 2012 & 2013 & 2014 & 2015 & & 2011 & 2012 & 2013 & 2014 & 2015 & & 2012 & 2013 & \\
\hline INBU & 0 & 1 & 0 & 0 & 0 & 1 & 0 & 1 & 0 & 0 & 0 & 1 & 0 & 1 & 0 & 0 & 0 & 1 & 0 & 0 & 0 \\
\hline BUOR & 6 & 3 & 2 & 0 & 1 & 12 & 6 & 3 & 2 & 0 & 1 & 12 & 6 & 3 & 2 & 0 & 1 & 12 & 0 & 0 & 0 \\
\hline
\end{tabular}


Table B2. Migrant capture rate by net and date during spring migration, Point Loma banding station, San Diego, California, 2011.

\begin{tabular}{|c|c|c|c|c|c|c|c|c|c|c|c|c|c|}
\hline \multirow{2}{*}{$\begin{array}{c}\text { Banding } \\
\text { day }\end{array}$} & \multirow[b]{2}{*}{ Date } & & \multicolumn{10}{|c|}{ Net } & \multirow{2}{*}{$\begin{array}{c}\text { Total } \\
\text { (hours:minutes) }\end{array}$} \\
\hline & & & 1 & 2 & 3 & 4 & 5 & 6 & 7 & 8 & 9 & 10 & \\
\hline \multirow[b]{3}{*}{1} & \multirow[b]{3}{*}{ 4-4-11 } & \multirow{3}{*}{$\begin{array}{l}\text { Net-hours (hours:minutes) } \\
\text { Captures (number) } \\
\text { Captures per net-hour }\end{array}$} & 3:00 & 3:00 & $3: 10$ & $3: 20$ & 3:00 & $2: 20$ & 0:00 & $2: 20$ & 3:00 & 3:00 & $26: 10$ \\
\hline & & & 1 & 1 & 0 & 3 & 1 & 1 & 0 & 1 & 0 & 0 & 8 \\
\hline & & & 0.33 & 0.33 & 0.00 & 0.90 & 0.33 & 0.43 & 0.00 & 0.43 & 0.00 & 0.00 & 0.31 \\
\hline \multirow[b]{3}{*}{2} & \multirow[b]{3}{*}{$4-5-11$} & \multirow{3}{*}{$\begin{array}{l}\text { Net-hours (hours:minutes) } \\
\text { Captures (number) } \\
\text { Captures per net-hour }\end{array}$} & $3: 00$ & 3:00 & 3:00 & 3:00 & 3:00 & 3:00 & 3:00 & 3:00 & 3:00 & 3:00 & $30: 00$ \\
\hline & & & 0 & 0 & 0 & 1 & 0 & 0 & 0 & 0 & 1 & 1 & 3 \\
\hline & & & 0.00 & 0.00 & 0.00 & 0.33 & 0.00 & 0.00 & 0.00 & 0.00 & 0.33 & 0.00 & 0.10 \\
\hline \multirow[b]{3}{*}{3} & \multirow[b]{3}{*}{$4-6-11$} & \multirow{3}{*}{$\begin{array}{l}\text { Net-hours (hours:minutes) } \\
\text { Captures (number) } \\
\text { Captures per net-hour }\end{array}$} & 3:00 & 3:00 & 3:00 & 3:00 & 3:00 & 3:00 & 3:00 & 3:00 & 3:00 & 3:00 & $30: 00$ \\
\hline & & & 2 & 0 & 0 & 1 & 0 & 3 & 1 & 0 & 1 & 1 & 9 \\
\hline & & & 0.67 & 0.00 & 0.00 & 0.33 & 0.00 & 1.00 & 0.33 & 0.00 & 0.33 & 0.33 & 0.30 \\
\hline \multirow[b]{3}{*}{4} & \multirow[b]{3}{*}{$4-7-11$} & \multirow{3}{*}{$\begin{array}{l}\text { Net-hours (hours:minutes) } \\
\text { Captures (number) } \\
\text { Captures per net-hour }\end{array}$} & $3: 00$ & $0: 40$ & 3:00 & $2: 20$ & $1: 00$ & 3:00 & $3: 00$ & $3: 10$ & $3: 10$ & 3:00 & $25: 20$ \\
\hline & & & 1 & 0 & 0 & 0 & 0 & 0 & 0 & 0 & 1 & 0 & 2 \\
\hline & & & 0.33 & 0.00 & 0.00 & 0.00 & 0.00 & 0.00 & 0.00 & 0.00 & 0.32 & 0.00 & 0.08 \\
\hline \multirow[b]{3}{*}{5} & \multirow[b]{3}{*}{$4-8-11$} & \multirow{3}{*}{$\begin{array}{l}\text { Net-hours (hours:minutes) } \\
\text { Captures (number) } \\
\text { Captures per net-hour }\end{array}$} & $3: 00$ & 3:00 & 3:00 & 0:00 & $1: 00$ & $0: 40$ & $1: 50$ & 3:00 & 0:00 & $2: 40$ & $18: 10$ \\
\hline & & & 1 & 0 & 0 & 0 & 0 & 0 & 0 & 0 & 0 & 0 & 1 \\
\hline & & & 0.33 & 0.00 & 0.00 & 0.00 & 0.00 & 0.00 & 0.00 & 0.00 & 0.00 & 0.00 & 0.06 \\
\hline \multirow[b]{3}{*}{6} & \multirow[b]{3}{*}{ 4-11-11 } & \multirow{3}{*}{$\begin{array}{l}\text { Net-hours (hours:minutes) } \\
\text { Captures (number) } \\
\text { Captures per net-hour }\end{array}$} & $3: 20$ & $3: 10$ & $3: 20$ & 3:00 & $3: 10$ & $3: 10$ & $3: 10$ & $3: 10$ & $3: 20$ & $3: 20$ & $32: 10$ \\
\hline & & & 1 & 1 & 0 & 2 & 1 & 2 & 2 & 0 & 2 & 1 & 12 \\
\hline & & & 0.30 & 0.32 & 0.00 & 0.67 & 0.32 & 0.63 & 0.63 & 0.00 & 0.60 & 0.30 & 0.37 \\
\hline \multirow[b]{3}{*}{7} & \multirow[b]{3}{*}{ 4-12-11 } & \multirow{3}{*}{$\begin{array}{l}\text { Net-hours (hours:minutes) } \\
\text { Captures (number) } \\
\text { Captures per net-hour }\end{array}$} & $3: 10$ & 3:00 & 3:00 & 3:00 & $3: 00$ & $3: 10$ & $3: 00$ & 3:00 & $3: 10$ & $3: 10$ & $30: 40$ \\
\hline & & & 1 & 0 & 0 & 1 & 0 & 2 & 0 & 0 & 0 & 0 & 4 \\
\hline & & & 0.32 & 0.00 & 0.00 & 0.33 & 0.00 & 0.63 & 0.00 & 0.00 & 0.00 & 0.00 & 0.13 \\
\hline \multirow[b]{3}{*}{8} & & Net-hours (hours:minutes) & $3: 10$ & 3:00 & $3: 00$ & $0: 00$ & $3: 10$ & $3: 20$ & $3: 00$ & $3: 00$ & $1: 10$ & $3: 20$ & $26: 10$ \\
\hline & & Captures (number) & 1 & 0 & 0 & 0 & 2 & 1 & 0 & 0 & 2 & 0 & 6 \\
\hline & 4-13-11 & Captures per net-hour & 0.32 & 0.00 & 0.00 & 0.00 & 0.63 & 0.30 & 0.00 & 0.00 & 1.71 & 0.00 & 0.23 \\
\hline & & Net-hours (hours:minutes) & $3: 00$ & $3: 00$ & $3: 00$ & $3: 10$ & $3: 10$ & $3: 10$ & $3: 00$ & $3: 00$ & $3: 00$ & $3: 00$ & $30: 30$ \\
\hline & & Captures (number) & 0 & 0 & 0 & 1 & 1 & 0 & 0 & 0 & 1 & 0 & 3 \\
\hline 9 & 4-14-11 & Captures per net-hour & 0.00 & 0.00 & 0.00 & 0.32 & 0.32 & 0.00 & 0.00 & 0.00 & 0.33 & 0.00 & 0.10 \\
\hline & & Net-hours (hours:minutes) & $3: 10$ & $3: 20$ & $3: 10$ & $3: 00$ & $3: 20$ & $3: 20$ & $3: 00$ & $3: 10$ & $3: 10$ & $3: 20$ & $32: 00$ \\
\hline & & Captures (number) & 2 & 3 & 3 & 2 & 1 & 5 & 2 & 0 & 1 & 0 & 19 \\
\hline 10 & $4-15-11$ & Captures per net-hour & 0.63 & 0.90 & 0.95 & 0.67 & 0.30 & 1.50 & 0.67 & 0.00 & 0.32 & 0.00 & 0.59 \\
\hline
\end{tabular}




\begin{tabular}{|c|c|c|c|c|c|c|c|c|c|c|c|c|c|}
\hline \multirow{2}{*}{$\begin{array}{c}\text { Banding } \\
\text { day }\end{array}$} & \multirow[b]{2}{*}{ Date } & & \multicolumn{10}{|c|}{ Net } & \multirow{2}{*}{$\begin{array}{c}\text { Total } \\
\text { (hours:minutes) }\end{array}$} \\
\hline & & & 1 & 2 & 3 & 4 & 5 & 6 & 7 & 8 & 9 & 10 & \\
\hline \multirow[b]{3}{*}{11} & \multirow[b]{3}{*}{$4-18-11$} & \multirow{3}{*}{$\begin{array}{l}\text { Net-hours (hours:minutes) } \\
\text { Captures (number) } \\
\text { Captures per net-hour }\end{array}$} & $3: 00$ & $2: 50$ & $3: 30$ & $3: 30$ & $2: 00$ & $3: 20$ & $3: 20$ & $3: 10$ & $2: 50$ & $3: 10$ & $30: 40$ \\
\hline & & & 1 & 0 & 1 & 0 & 0 & 0 & 0 & 0 & 0 & 0 & 2 \\
\hline & & & 0.33 & 0.00 & 0.29 & 0.00 & 0.00 & 0.00 & 0.00 & 0.00 & 0.00 & 0.00 & 0.07 \\
\hline \multirow[b]{3}{*}{12} & \multirow[b]{3}{*}{$4-19-11$} & \multirow{3}{*}{$\begin{array}{l}\text { Net-hours (hours:minutes) } \\
\text { Captures (number) } \\
\text { Captures per net-hour }\end{array}$} & $3: 10$ & $3: 10$ & 3:00 & $3: 10$ & $3: 00$ & $3: 10$ & $3: 00$ & $3: 10$ & $3: 20$ & $3: 10$ & $31: 20$ \\
\hline & & & 0 & 0 & 0 & 1 & 0 & 0 & 0 & 0 & 0 & 0 & 1 \\
\hline & & & 0.00 & 0.00 & 0.00 & 0.32 & 0.00 & 0.00 & 0.00 & 0.00 & 0.00 & 0.00 & 0.03 \\
\hline \multirow[b]{3}{*}{13} & \multirow[b]{3}{*}{$4-20-11$} & \multirow{3}{*}{$\begin{array}{l}\text { Net-hours (hours:minutes) } \\
\text { Captures (number) } \\
\text { Captures per net-hour }\end{array}$} & $3: 20$ & $3: 30$ & $3: 40$ & $3: 40$ & $2: 50$ & $3: 10$ & $3: 10$ & $3: 20$ & $3: 20$ & $3: 20$ & $33: 20$ \\
\hline & & & 3 & 0 & 1 & 4 & 0 & 2 & 3 & 1 & 2 & 3 & 19 \\
\hline & & & 0.90 & 0.00 & 0.27 & 1.09 & 0.00 & 0.63 & 0.95 & 0.30 & 0.60 & 0.90 & 0.57 \\
\hline \multirow[b]{3}{*}{14} & \multirow[b]{3}{*}{ 4-21-11 } & \multirow{3}{*}{$\begin{array}{l}\text { Net-hours (hours:minutes) } \\
\text { Captures (number) } \\
\text { Captures per net-hour }\end{array}$} & $3: 00$ & $3: 00$ & $3: 00$ & $2: 50$ & $2: 40$ & $2: 40$ & $3: 00$ & $3: 10$ & $3: 00$ & $3: 00$ & $29: 20$ \\
\hline & & & 4 & 1 & 6 & 4 & 4 & 8 & 3 & 1 & 2 & 6 & 39 \\
\hline & & & 1.33 & 0.33 & 2.00 & 1.41 & 1.50 & 3.00 & 1.00 & 0.32 & 0.67 & 2.00 & 1.33 \\
\hline \multirow[b]{3}{*}{15} & \multirow[b]{3}{*}{$4-22-11$} & \multirow{3}{*}{$\begin{array}{l}\text { Net-hours (hours:minutes) } \\
\text { Captures (number) } \\
\text { Captures per net-hour }\end{array}$} & $3: 00$ & $3: 00$ & 3:00 & $3: 20$ & $3: 10$ & $3: 20$ & $3: 20$ & $3: 20$ & $3: 00$ & $3: 10$ & $31: 40$ \\
\hline & & & 4 & 2 & 2 & 3 & 0 & 0 & 2 & 2 & 2 & 0 & 17 \\
\hline & & & 1.33 & 0.67 & 0.67 & 0.90 & 0.00 & 0.00 & 0.60 & 0.60 & 0.67 & 0.00 & 0.54 \\
\hline \multirow[b]{3}{*}{16} & \multirow[b]{3}{*}{$4-25-11$} & \multirow{3}{*}{$\begin{array}{l}\text { Net-hours (hours:minutes) } \\
\text { Captures (number) } \\
\text { Captures per net-hour }\end{array}$} & $3: 20$ & $3: 20$ & $3: 20$ & $3: 20$ & $2: 50$ & $3: 00$ & $3: 00$ & $2: 40$ & $3: 20$ & $3: 20$ & $31: 30$ \\
\hline & & & 0 & 0 & 1 & 4 & 0 & 1 & 0 & 1 & 1 & 0 & 8 \\
\hline & & & 0.00 & 0.00 & 0.30 & 1.20 & 0.00 & 0.33 & 0.00 & 0.38 & 0.30 & 0.00 & 0.25 \\
\hline \multirow[b]{3}{*}{17} & \multirow[b]{3}{*}{$4-26-11$} & \multirow{3}{*}{$\begin{array}{l}\text { Net-hours (hours:minutes) } \\
\text { Captures (number) } \\
\text { Captures per net-hour }\end{array}$} & $3: 50$ & $3: 10$ & $3: 10$ & $3: 20$ & $3: 30$ & 1:00 & $3: 30$ & $3: 20$ & 4:00 & 3:00 & $31: 50$ \\
\hline & & & 2 & 3 & 4 & 6 & 2 & 2 & 7 & 2 & 1 & 2 & 31 \\
\hline & & & 0.52 & 0.95 & 1.26 & 1.80 & 0.57 & 2.00 & 2.00 & 0.60 & 0.25 & 0.67 & 0.97 \\
\hline & & Net-hours (hours:minutes) & $3: 30$ & $3: 10$ & $3: 20$ & $3: 20$ & $4: 00$ & $4: 10$ & $4: 00$ & $4: 10$ & $3: 20$ & $3: 20$ & $36: 20$ \\
\hline & & Captures (number) & 3 & 7 & 3 & 8 & 7 & 15 & 7 & 3 & 10 & 2 & $66^{1}$ \\
\hline 18 & $4-27-11$ & Captures per net-hour & 0.86 & 2.21 & 0.90 & 2.40 & 1.75 & 3.60 & 1.75 & 0.72 & 3.00 & 0.60 & 1.82 \\
\hline & & Net-hours (hours:minutes) & $3: 10$ & $2: 30$ & 3:00 & $3: 10$ & $3: 00$ & $3: 10$ & $3: 00$ & $3: 10$ & $3: 00$ & $3: 00$ & $30: 10$ \\
\hline & & Captures (number) & 1 & 2 & 2 & 5 & 0 & 1 & 2 & 6 & 5 & 3 & 27 \\
\hline 19 & $4-28-11$ & Captures per net-hour & 0.32 & 0.80 & 0.67 & 1.58 & 0.00 & 0.32 & 0.67 & 1.89 & 1.67 & 1.00 & 0.90 \\
\hline & & Net-hours (hours:minutes) & $3: 20$ & $3: 10$ & $3: 20$ & 3:00 & $2: 00$ & 3:00 & $3: 20$ & $3: 20$ & $3: 20$ & $3: 40$ & $31: 30$ \\
\hline & & Captures (number) & 2 & 0 & 1 & 4 & 0 & 1 & 0 & 0 & 3 & 0 & 11 \\
\hline 20 & $4-29-11$ & Captures per net-hour & 0.60 & 0.00 & 0.30 & 1.33 & 0.00 & 0.33 & 0.00 & 0.00 & 0.90 & 0.00 & 0.35 \\
\hline & & Net-hours (hours:minutes) & $5: 30$ & $4: 50$ & $4: 50$ & $5: 10$ & $5: 10$ & $5: 00$ & $4: 10$ & $5: 20$ & $5: 40$ & $5: 40$ & $51: 20$ \\
\hline & & Captures (number) & 2 & 1 & 1 & 6 & 2 & 1 & 2 & 2 & 4 & 4 & 25 \\
\hline 21 & $5-5-11$ & Captures per net-hour & 0.36 & 0.21 & 0.21 & 1.16 & 0.39 & 0.20 & 0.48 & 0.38 & 0.71 & 0.71 & 0.49 \\
\hline
\end{tabular}




\begin{tabular}{|c|c|c|c|c|c|c|c|c|c|c|c|c|c|}
\hline \multirow{2}{*}{$\begin{array}{c}\text { Banding } \\
\text { day }\end{array}$} & \multirow[b]{2}{*}{ Date } & & \multicolumn{10}{|c|}{ Net } & \multirow{2}{*}{$\begin{array}{c}\text { Total } \\
\text { (hours:minutes) }\end{array}$} \\
\hline & & & 1 & 2 & 3 & 4 & 5 & 6 & 7 & 8 & 9 & 10 & \\
\hline \multirow{2}{*}{22} & \multirow{2}{*}{$5-12-11$} & \multirow{2}{*}{$\begin{array}{l}\text { Net-hours (hours:minutes) } \\
\text { Captures (number) } \\
\text { Captures per net-hour }\end{array}$} & $5: 10$ & $5: 20$ & $5: 10$ & $5: 00$ & $4: 40$ & $4: 50$ & $2: 50$ & $4: 20$ & $5: 00$ & $4: 30$ & $46: 50$ \\
\hline & & & 6 & 8 & 4 & 12 & 9 & 6 & 8 & 4 & 4 & 6 & 67 \\
\hline \multirow[b]{3}{*}{23} & \multirow[b]{3}{*}{$5-26-11$} & \multirow{3}{*}{$\begin{array}{l}\text { Net-hours (hours:minutes) } \\
\text { Captures (number) } \\
\text { Captures per net-hour }\end{array}$} & $5: 00$ & $5: 10$ & $4: 50$ & $5: 10$ & $4: 50$ & $4: 50$ & $4: 40$ & $4: 50$ & $5: 00$ & $5: 00$ & $49: 20$ \\
\hline & & & 1 & 4 & 1 & 7 & 3 & 1 & 3 & 1 & 6 & 0 & 27 \\
\hline & & & 0.20 & 0.77 & 0.21 & 1.35 & 0.62 & 0.21 & 0.64 & 0.21 & 1.20 & 0.00 & 0.55 \\
\hline \multicolumn{2}{|c|}{$\begin{array}{c}\text { Totals by } \\
\text { net }\end{array}$} & $\begin{array}{l}\text { Net-hours (hours:minutes) } \\
\text { Captures (number) } \\
\text { Captures per net-hour }\end{array}$ & 0.49 & 0.44 & 0.39 & 1.04 & 0.47 & 0.71 & 0.60 & 0.31 & 0.66 & 0.37 & 0.54 \\
\hline
\end{tabular}

${ }^{\mathrm{I}}$ Total adjusted for one capture with unknown net number 
Table B3. Migrant capture rate by net and date during spring migration, Point Loma banding station, San Diego, California, 2012.

\begin{tabular}{|c|c|c|c|c|c|c|c|c|c|c|c|c|c|}
\hline \multirow{2}{*}{$\begin{array}{c}\text { Banding } \\
\text { day }\end{array}$} & \multirow[b]{2}{*}{ Date } & & \multicolumn{10}{|c|}{ Net } & \multirow{2}{*}{$\begin{array}{c}\text { Total } \\
\text { (hours:minutes) }\end{array}$} \\
\hline & & & 1 & 2 & 3 & 4 & 5 & 6 & 7 & 8 & 9 & 10 & \\
\hline \multirow[b]{3}{*}{1} & \multirow[b]{3}{*}{$4-02-12$} & \multirow{3}{*}{$\begin{array}{l}\text { Net-hours (hours:minutes) } \\
\text { Captures (number) } \\
\text { Captures per net-hour }\end{array}$} & $2: 50$ & $3: 00$ & $2: 50$ & $2: 50$ & $2: 50$ & $3: 00$ & $3: 00$ & $3: 10$ & $2: 40$ & $2: 50$ & $29: 00$ \\
\hline & & & 0 & 0 & 0 & 1 & 0 & 1 & 1 & 1 & 0 & 2 & 6 \\
\hline & & & 0.00 & 0.00 & 0.00 & 0.35 & 0.00 & 0.33 & 0.33 & 0.32 & 0.00 & 0.71 & 0.21 \\
\hline \multirow[b]{3}{*}{2} & \multirow[b]{3}{*}{ 4-03-12 } & \multirow{3}{*}{$\begin{array}{l}\text { Net-hours (hours:minutes) } \\
\text { Captures (number) } \\
\text { Captures per net-hour }\end{array}$} & $3: 00$ & $3: 00$ & $2: 50$ & $3: 10$ & $3: 00$ & $3: 00$ & $3: 00$ & $3: 10$ & $3: 00$ & $3: 00$ & $30: 10$ \\
\hline & & & 1 & 0 & 0 & 3 & 0 & 1 & 0 & 0 & 2 & 1 & 8 \\
\hline & & & 0.33 & 0.00 & 0.00 & 0.95 & 0.00 & 0.33 & 0.00 & 0.00 & 0.67 & 0.33 & 0.27 \\
\hline \multirow[b]{3}{*}{3} & \multirow[b]{3}{*}{ 4-04-12 } & \multirow{3}{*}{$\begin{array}{l}\text { Net-hours (hours:minutes) } \\
\text { Captures (number) } \\
\text { Captures per net-hour }\end{array}$} & $2: 50$ & $3: 00$ & $2: 50$ & $3: 10$ & $2: 50$ & $3: 10$ & $2: 50$ & $2: 40$ & $3: 10$ & $3: 00$ & $29: 30$ \\
\hline & & & 0 & 1 & 1 & 1 & 0 & 1 & 0 & 0 & 1 & 3 & 8 \\
\hline & & & 0.00 & 0.33 & 0.35 & 0.32 & 0.00 & 0.32 & 0.00 & 0.00 & 0.32 & 1.00 & 0.27 \\
\hline \multirow[b]{3}{*}{4} & \multirow[b]{3}{*}{ 4-05-12 } & \multirow{3}{*}{$\begin{array}{l}\text { Net-hours (hours:minutes) } \\
\text { Captures (number) } \\
\text { Captures per net-hour }\end{array}$} & $2: 40$ & $3: 00$ & $3: 00$ & $3: 00$ & $2: 40$ & $2: 50$ & $2: 50$ & $2: 50$ & $2: 40$ & $2: 40$ & $28: 10$ \\
\hline & & & 0 & 0 & 1 & 2 & 0 & 1 & 0 & 0 & 1 & 0 & 5 \\
\hline & & & 0.00 & 0.00 & 0.33 & 0.67 & 0.00 & 0.35 & 0.00 & 0.00 & 0.38 & 0.00 & 0.18 \\
\hline \multirow[b]{3}{*}{5} & \multirow[b]{3}{*}{$4-06-12$} & \multirow{3}{*}{$\begin{array}{l}\text { Net-hours (hours:minutes) } \\
\text { Captures (number) } \\
\text { Captures per net-hour }\end{array}$} & $3: 00$ & $3: 00$ & $3: 00$ & $3: 00$ & $3: 30$ & $3: 10$ & $3: 10$ & $3: 20$ & $3: 00$ & $3: 00$ & $31: 10$ \\
\hline & & & 1 & 1 & 0 & 0 & 0 & 1 & 1 & 1 & 1 & 2 & 8 \\
\hline & & & 0.33 & 0.33 & 0.00 & 0.00 & 0.00 & 0.32 & 0.32 & 0.30 & 0.33 & 0.67 & 0.26 \\
\hline \multirow[b]{3}{*}{6} & \multirow[b]{3}{*}{$4-09-12$} & \multirow{3}{*}{$\begin{array}{l}\text { Net-hours (hours:minutes) } \\
\text { Captures (number) } \\
\text { Captures per net-hour }\end{array}$} & $2: 50$ & $2: 50$ & $3: 00$ & $3: 00$ & $3: 10$ & $3: 00$ & $3: 10$ & $3: 00$ & $3: 10$ & $3: 20$ & $30: 30$ \\
\hline & & & 0 & 1 & 1 & 1 & 1 & 0 & 2 & 1 & 2 & 0 & 9 \\
\hline & & & 0.00 & 0.35 & 0.33 & 0.33 & 0.32 & 0.00 & 0.63 & 0.33 & 0.63 & 0.00 & 0.30 \\
\hline \multirow[b]{3}{*}{7} & \multirow[b]{3}{*}{$4-10-12$} & \multirow{3}{*}{$\begin{array}{l}\text { Net-hours (hours:minutes) } \\
\text { Captures (number) } \\
\text { Captures per net-hour }\end{array}$} & $3: 00$ & $2: 40$ & $2: 50$ & $2: 50$ & $3: 00$ & $3: 00$ & $2: 50$ & $3: 00$ & $3: 00$ & $3: 00$ & $29: 10$ \\
\hline & & & 0 & 0 & 0 & 2 & 0 & 2 & 2 & 3 & 1 & 1 & 11 \\
\hline & & & 0.00 & 0.00 & 0.00 & 0.71 & 0.00 & 0.67 & 0.71 & 1.00 & 0.33 & 0.33 & 0.38 \\
\hline \multirow[b]{3}{*}{8} & & Net-hours (hours:minutes) & $3: 10$ & $3: 10$ & $3: 10$ & $3: 10$ & $3: 10$ & $3: 10$ & $3: 00$ & $3: 10$ & $3: 20$ & $3: 10$ & $31: 40$ \\
\hline & & Captures (number) & 0 & 0 & 0 & 0 & 0 & 0 & 0 & 0 & 0 & 0 & 0 \\
\hline & $4-11-12$ & Captures per net-hour & 0.00 & 0.00 & 0.00 & 0.00 & 0.00 & 0.00 & 0.00 & 0.00 & 0.00 & 0.00 & 0.00 \\
\hline & & Net-hours (hours:minutes) & $3: 20$ & $3: 10$ & $3: 10$ & $3: 00$ & $3: 00$ & $3: 00$ & $3: 00$ & $3: 10$ & $3: 20$ & $3: 30$ & $31: 40$ \\
\hline & & Captures (number) & 0 & 0 & 0 & 0 & 0 & 0 & 0 & 0 & 1 & 2 & 3 \\
\hline 9 & 4-12-12 & Captures per net-hour & 0.00 & 0.00 & 0.00 & 0.00 & 0.00 & 0.00 & 0.00 & 0.00 & 0.30 & 0.57 & 0.09 \\
\hline & & Net-hours (hours:minutes) & $2: 50$ & $3: 20$ & $2: 50$ & $3: 00$ & $3: 00$ & $3: 00$ & $3: 00$ & $3: 00$ & $2: 50$ & $2: 40$ & $29: 30$ \\
\hline & & Captures (number) & 0 & 1 & 0 & 1 & 0 & 0 & 0 & 0 & 0 & 0 & 2 \\
\hline 10 & $4-13-12$ & Captures per net-hour & 0.00 & 0.30 & 0.00 & 0.33 & 0.00 & 0.00 & 0.00 & 0.00 & 0.00 & 0.00 & 0.07 \\
\hline
\end{tabular}




\begin{tabular}{|c|c|c|c|c|c|c|c|c|c|c|c|c|c|}
\hline \multirow{2}{*}{$\begin{array}{l}\text { Banding } \\
\text { day }\end{array}$} & \multirow[b]{2}{*}{ Date } & & \multicolumn{10}{|c|}{ Net } & \multirow{2}{*}{$\begin{array}{c}\text { Total } \\
\text { (hours:minutes) }\end{array}$} \\
\hline & & & 1 & 2 & 3 & 4 & 5 & 6 & 7 & 8 & 9 & 10 & \\
\hline \multirow[b]{3}{*}{11} & \multirow[b]{3}{*}{ 4-16-12 } & \multirow{3}{*}{$\begin{array}{l}\text { Net-hours (hours:minutes) } \\
\text { Captures (number) } \\
\text { Captures per net-hour }\end{array}$} & $3: 10$ & $3: 10$ & $3: 00$ & $3: 10$ & $3: 10$ & $3: 10$ & $3: 20$ & $3: 10$ & $3: 10$ & $3: 20$ & $31: 50$ \\
\hline & & & 2 & 1 & 1 & 6 & 3 & 1 & 2 & 0 & 1 & 1 & 18 \\
\hline & & & 0.63 & 0.32 & 0.33 & 1.89 & 0.95 & 0.32 & 0.60 & 0.00 & 0.32 & 0.30 & 0.57 \\
\hline \multirow[b]{3}{*}{12} & \multirow[b]{3}{*}{$4-17-12$} & \multirow{3}{*}{$\begin{array}{l}\text { Net-hours (hours:minutes) } \\
\text { Captures (number) } \\
\text { Captures per net-hour }\end{array}$} & $3: 10$ & $3: 10$ & $3: 10$ & $3: 10$ & $3: 10$ & $3: 20$ & $3: 00$ & $3: 20$ & $3: 10$ & $3: 20$ & $32: 00$ \\
\hline & & & 1 & 1 & 2 & 4 & 1 & 7 & 7 & 1 & 3 & 1 & 28 \\
\hline & & & 0.32 & 0.32 & 0.63 & 1.26 & 0.32 & 2.10 & 2.33 & 0.30 & 0.95 & 0.30 & 0.88 \\
\hline \multirow[b]{3}{*}{13} & \multirow[b]{3}{*}{$4-18-12$} & \multirow{3}{*}{$\begin{array}{l}\text { Net-hours (hours:minutes) } \\
\text { Captures (number) } \\
\text { Captures per net-hour }\end{array}$} & $3: 10$ & $3: 10$ & $3: 10$ & $3: 10$ & $3: 00$ & $2: 50$ & $3: 10$ & $3: 00$ & $3: 00$ & $3: 10$ & $30: 50$ \\
\hline & & & 2 & 0 & 0 & 1 & 1 & 0 & 0 & 0 & 1 & 1 & 6 \\
\hline & & & 0.63 & 0.00 & 0.00 & 0.32 & 0.33 & 0.00 & 0.00 & 0.00 & 0.33 & 0.32 & 0.19 \\
\hline \multirow[b]{3}{*}{14} & \multirow[b]{3}{*}{ 4-19-12 } & \multirow{3}{*}{$\begin{array}{l}\text { Net-hours (hours:minutes) } \\
\text { Captures (number) } \\
\text { Captures per net-hour }\end{array}$} & $3: 10$ & $3: 10$ & $3: 10$ & $3: 10$ & $3: 00$ & $3: 00$ & 3:00 & $3: 10$ & $3: 10$ & $3: 20$ & $31: 20$ \\
\hline & & & 0 & 1 & 2 & 0 & 1 & 0 & 0 & 2 & 0 & 2 & 8 \\
\hline & & & 0.00 & 0.32 & 0.63 & 0.00 & 0.33 & 0.00 & 0.00 & 0.63 & 0.00 & 0.60 & 0.26 \\
\hline \multirow[b]{3}{*}{15} & \multirow[b]{3}{*}{$4-20-12$} & \multirow{3}{*}{$\begin{array}{l}\text { Net-hours (hours:minutes) } \\
\text { Captures (number) } \\
\text { Captures per net-hour }\end{array}$} & $3: 10$ & $3: 10$ & 3:00 & $3: 10$ & $3: 10$ & $3: 10$ & $3: 10$ & $3: 10$ & $3: 00$ & $3: 20$ & $31: 30$ \\
\hline & & & 0 & 0 & 2 & 2 & 3 & 2 & 3 & 1 & 3 & 3 & 19 \\
\hline & & & 0.00 & 0.00 & 0.67 & 0.63 & 0.95 & 0.63 & 0.95 & 0.32 & 1.00 & 0.90 & 0.60 \\
\hline \multirow{5}{*}{16} & \multirow{5}{*}{$4-23-12$} & \multirow{3}{*}{$\begin{array}{l}\text { Net-hours (hours:minutes) } \\
\text { Captures (number) } \\
\text { Captures per net-hour }\end{array}$} & $3: 20$ & $3: 00$ & $3: 20$ & $3: 20$ & $3: 10$ & $3: 20$ & $3: 00$ & $3: 10$ & $3: 30$ & $3: 30$ & $32: 40$ \\
\hline & & & 0 & 0 & 1 & 5 & 1 & 1 & 1 & 0 & 2 & 7 & 18 \\
\hline & & & 0.00 & 0.00 & 0.30 & 1.50 & 0.32 & 0.30 & 0.33 & 0.00 & 0.57 & 2.00 & 0.55 \\
\hline & & \multirow{2}{*}{$\begin{array}{l}\text { Net-hours (hours:minutes) } \\
\text { Captures (number) }\end{array}$} & $3: 10$ & $3: 20$ & $3: 10$ & $3: 10$ & $3: 10$ & $3: 10$ & $3: 10$ & $3: 10$ & 3:00 & 3:00 & $31: 30$ \\
\hline & & & 0 & 2 & 1 & 2 & 2 & 0 & 0 & 0 & 2 & 2 & 11 \\
\hline 17 & $4-24-12$ & Captures per net-hour & 0.00 & 0.60 & 0.32 & 0.63 & 0.63 & 0.00 & 0.00 & 0.00 & 0.67 & 0.67 & 0.35 \\
\hline \multirow[b]{3}{*}{18} & \multirow[b]{3}{*}{$4-25-12$} & Net-hours (hours:minutes) & $1: 00$ & $1: 10$ & $0: 50$ & 1:00 & $1: 10$ & $1: 10$ & $0: 50$ & 1:00 & $1: 00$ & $1: 00$ & $10: 10$ \\
\hline & & Captures (number) & 0 & 0 & 0 & 0 & 0 & 2 & 2 & 0 & 2 & 2 & 8 \\
\hline & & Captures per net-hour & 0.00 & 0.00 & 0.00 & 0.00 & 0.00 & 1.71 & 2.40 & 0.00 & 2.00 & 2.00 & 0.79 \\
\hline & & Net-hours (hours:minutes) & $2: 50$ & $3: 10$ & $3: 00$ & $2: 50$ & $3: 10$ & 3:00 & $3: 00$ & $3: 00$ & $2: 50$ & $2: 50$ & $29: 40$ \\
\hline & & Captures (number) & 0 & 0 & 0 & 2 & 0 & 1 & 0 & 0 & 0 & 0 & 3 \\
\hline 19 & $4-27-12$ & Captures per net-hour & 0.00 & 0.00 & 0.00 & 0.71 & 0.00 & 0.33 & 0.00 & 0.00 & 0.00 & 0.00 & 0.10 \\
\hline & & Net-hours (hours:minutes) & $5: 10$ & $5: 10$ & $5: 10$ & $5: 10$ & $5: 10$ & $5: 10$ & $5: 10$ & $5: 10$ & $5: 10$ & $5: 10$ & $51: 40$ \\
\hline & & Captures (number) & 2 & 6 & 3 & 9 & 5 & 8 & 7 & 9 & 4 & 4 & 57 \\
\hline 20 & $5-08-12$ & Captures per net-hour & 0.39 & 1.16 & 0.58 & 1.74 & 0.97 & 1.55 & 1.35 & 1.74 & 0.77 & 0.77 & 1.10 \\
\hline & & Net-hours (hours:minutes) & 5:00 & $5: 10$ & $5: 10$ & $5: 10$ & $5: 10$ & 5:00 & $5: 20$ & $5: 10$ & 5:00 & 5:00 & $51: 10$ \\
\hline & & Captures (number) & 2 & 2 & 1 & 8 & 3 & 3 & 4 & 5 & 8 & 5 & 41 \\
\hline 21 & $5-15-12$ & Captures per net-hour & 0.40 & 0.39 & 0.19 & 1.55 & 0.58 & 0.60 & 0.75 & 0.97 & 1.60 & 1.00 & 0.80 \\
\hline
\end{tabular}




\begin{tabular}{|c|c|c|c|c|c|c|c|c|c|c|c|c|c|}
\hline \multirow{2}{*}{$\begin{array}{l}\text { Banding } \\
\text { day }\end{array}$} & \multirow[b]{2}{*}{ Date } & & \multicolumn{10}{|c|}{ Net } & \multirow{2}{*}{$\begin{array}{c}\text { Total } \\
\text { (hours:minutes) }\end{array}$} \\
\hline & & & 1 & 2 & 3 & 4 & 5 & 6 & 7 & 8 & 9 & 10 & \\
\hline \multirow{2}{*}{22} & \multirow{2}{*}{$5-29-12$} & \multirow{2}{*}{$\begin{array}{l}\text { Net-hours (hours:minutes) } \\
\text { Captures (number) } \\
\text { Captures per net-hour }\end{array}$} & 5:00 & 5:00 & 4:50 & 5:00 & $5: 00$ & $5: 00$ & 5:00 & $5: 00$ & $5: 10$ & 5:00 & $50: 00$ \\
\hline & & & 1 & 2 & 4 & 2 & 0 & 2 & 1 & 1 & 3 & 1 & 17 \\
\hline \multirow{3}{*}{\multicolumn{2}{|c|}{$\begin{array}{c}\text { Totals by } \\
\text { net }\end{array}$}} & \multirow{3}{*}{$\begin{array}{l}\text { Net-hours (hours:minutes) } \\
\text { Captures (number) } \\
\text { Captures per net-hour }\end{array}$} & $70: 50$ & $72: 00$ & $70: 30$ & $71: 40$ & $71: 40$ & $71: 40$ & $71: 00$ & $72: 00$ & $71: 20$ & $72: 10$ & $714: 50$ \\
\hline & & & 12 & 19 & 20 & 52 & 21 & 34 & 33 & 25 & 38 & 40 & 294 \\
\hline & & & 0.17 & 0.26 & 0.28 & 0.73 & 0.29 & 0.47 & 0.46 & 0.35 & 0.53 & 0.55 & 0.41 \\
\hline
\end{tabular}


Table B4. Migrant capture rate by net and date during spring migration, Point Loma banding station, San Diego, California, 2013.

\begin{tabular}{|c|c|c|c|c|c|c|c|c|c|c|c|c|c|}
\hline \multirow{2}{*}{$\begin{array}{l}\text { Banding } \\
\text { day }\end{array}$} & \multirow[b]{2}{*}{ Date } & & \multicolumn{10}{|c|}{ Net } & \multirow{2}{*}{$\begin{array}{c}\text { Total } \\
\text { (hours:minutes) }\end{array}$} \\
\hline & & & 1 & 2 & 3 & 4 & 5 & 6 & 7 & 8 & 9 & 10 & \\
\hline \multirow[b]{3}{*}{1} & \multirow[b]{3}{*}{$4-01-13$} & \multirow{3}{*}{$\begin{array}{l}\text { Net-hours (hours:minutes) } \\
\text { Captures (number) } \\
\text { Captures per net-hour }\end{array}$} & $3: 10$ & $3: 00$ & $3: 30$ & $3: 10$ & $3: 10$ & $3: 10$ & $3: 10$ & $3: 10$ & $3: 00$ & $3: 00$ & $31: 30$ \\
\hline & & & 0 & 3 & 2 & 0 & 2 & 0 & 0 & 0 & 0 & 0 & 7 \\
\hline & & & 0.00 & 1.00 & 0.57 & 0.00 & 0.63 & 0.00 & 0.00 & 0.00 & 0.00 & 0.00 & 0.22 \\
\hline \multirow[b]{3}{*}{2} & \multirow[b]{3}{*}{ 4-02-13 } & \multirow{3}{*}{$\begin{array}{l}\text { Net-hours (hours:minutes) } \\
\text { Captures (number) } \\
\text { Captures per net-hour }\end{array}$} & $3: 10$ & $3: 20$ & $3: 00$ & $3: 20$ & $3: 00$ & $3: 00$ & $3: 10$ & $3: 00$ & $3: 00$ & $3: 00$ & $31: 00$ \\
\hline & & & 1 & 1 & 0 & 1 & 0 & 1 & 0 & 0 & 0 & 0 & 4 \\
\hline & & & 0.32 & 0.30 & 0.00 & 0.30 & 0.00 & 0.33 & 0.00 & 0.00 & 0.00 & 0.00 & 0.13 \\
\hline \multirow[b]{3}{*}{3} & \multirow[b]{3}{*}{ 4-03-13 } & \multirow{3}{*}{$\begin{array}{l}\text { Net-hours (hours:minutes) } \\
\text { Captures (number) } \\
\text { Captures per net-hour }\end{array}$} & $3: 00$ & $3: 20$ & $3: 10$ & $3: 10$ & $2: 50$ & $3: 00$ & $3: 00$ & $2: 50$ & $3: 10$ & $3: 00$ & $30: 30$ \\
\hline & & & 0 & 0 & 3 & 1 & 1 & 1 & 0 & 1 & 2 & 2 & 11 \\
\hline & & & 0.00 & 0.00 & 0.95 & 0.32 & 0.35 & 0.33 & 0.00 & 0.35 & 0.63 & 0.67 & 0.36 \\
\hline \multirow[b]{3}{*}{4} & \multirow[b]{3}{*}{ 4-04-13 } & \multirow{3}{*}{$\begin{array}{l}\text { Net-hours (hours:minutes) } \\
\text { Captures (number) } \\
\text { Captures per net-hour }\end{array}$} & $2: 50$ & $2: 50$ & $3: 00$ & $3: 00$ & $2: 50$ & $2: 50$ & $3: 00$ & $3: 00$ & $2: 50$ & $2: 40$ & $28: 50$ \\
\hline & & & 2 & 2 & 2 & 2 & 1 & 2 & 0 & 1 & 0 & 0 & 12 \\
\hline & & & 0.71 & 0.71 & 0.67 & 0.67 & 0.35 & 0.71 & 0.00 & 0.33 & 0.00 & 0.00 & 0.42 \\
\hline \multirow[b]{3}{*}{5} & \multirow[b]{3}{*}{$4-05-13$} & \multirow{3}{*}{$\begin{array}{l}\text { Net-hours (hours:minutes) } \\
\text { Captures (number) } \\
\text { Captures per net-hour }\end{array}$} & $3: 20$ & $3: 20$ & $3: 00$ & $2: 40$ & $3: 10$ & $3: 10$ & $3: 10$ & $3: 10$ & $3: 20$ & $3: 20$ & $31: 40$ \\
\hline & & & 2 & 3 & 0 & 0 & 2 & 2 & 1 & 0 & 2 & 0 & 12 \\
\hline & & & 0.60 & 0.90 & 0.00 & 0.00 & 0.63 & 0.63 & 0.32 & 0.00 & 0.60 & 0.00 & 0.38 \\
\hline \multirow[b]{3}{*}{6} & \multirow[b]{3}{*}{$4-08-13$} & \multirow{3}{*}{$\begin{array}{l}\text { Net-hours (hours:minutes) } \\
\text { Captures (number) } \\
\text { Captures per net-hour }\end{array}$} & $3: 00$ & $3: 10$ & $3: 10$ & $1: 20$ & $3: 10$ & $2: 00$ & $3: 00$ & $3: 10$ & $0: 20$ & $2: 00$ & $24: 20$ \\
\hline & & & 0 & 3 & 0 & 0 & 0 & 0 & 1 & 0 & 0 & 0 & 4 \\
\hline & & & 0.00 & 0.95 & 0.00 & 0.00 & 0.00 & 0.00 & 0.33 & 0.00 & 0.00 & 0.00 & 0.16 \\
\hline \multirow[b]{3}{*}{7} & \multirow[b]{3}{*}{$4-09-13$} & \multirow{3}{*}{$\begin{array}{l}\text { Net-hours (hours:minutes) } \\
\text { Captures (number) } \\
\text { Captures per net-hour }\end{array}$} & $3: 00$ & $3: 10$ & $2: 50$ & $3: 30$ & $3: 10$ & $3: 00$ & $3: 00$ & $3: 10$ & $3: 10$ & $3: 10$ & $31: 10$ \\
\hline & & & 0 & 0 & 0 & 0 & 0 & 0 & 1 & 0 & 0 & 0 & 1 \\
\hline & & & 0.00 & 0.00 & 0.00 & 0.00 & 0.00 & 0.00 & 0.33 & 0.00 & 0.00 & 0.00 & 0.03 \\
\hline \multirow[b]{3}{*}{8} & & Net-hours (hours:minutes) & $3: 00$ & $3: 10$ & $3: 00$ & $3: 00$ & $3: 10$ & $3: 10$ & $3: 00$ & $3: 00$ & $2: 50$ & $3: 00$ & $30: 20$ \\
\hline & & Captures (number) & 0 & 0 & 1 & 1 & 3 & 2 & 2 & 0 & 4 & 1 & 14 \\
\hline & $4-10-13$ & Captures per net-hour & 0.00 & 0.00 & 0.33 & 0.33 & 0.95 & 0.63 & 0.67 & 0.00 & 1.41 & 0.33 & 0.46 \\
\hline & & Net-hours (hours:minutes) & $3: 10$ & $3: 10$ & $3: 00$ & $3: 10$ & $3: 00$ & $3: 00$ & $3: 10$ & $3: 00$ & $3: 10$ & $3: 20$ & $31: 10$ \\
\hline & & Captures (number) & 0 & 1 & 6 & 2 & 0 & 2 & 2 & 0 & 4 & 1 & 201 \\
\hline 9 & 4-11-13 & Captures per net-hour & 0.00 & 0.32 & 2.00 & 0.63 & 0.00 & 0.67 & 0.63 & 0.00 & 1.26 & 0.30 & 0.64 \\
\hline & & Net-hours (hours:minutes) & $2: 50$ & $3: 00$ & $3: 00$ & $3: 30$ & $2: 50$ & $3: 10$ & $3: 00$ & $3: 10$ & $2: 50$ & $2: 50$ & $30: 10$ \\
\hline & & Captures (number) & 3 & 2 & 2 & 7 & 2 & 1 & 0 & 0 & 5 & 1 & 23 \\
\hline 10 & $4-12-13$ & Captures per net-hour & 1.06 & 0.67 & 0.67 & 2.00 & 0.71 & 0.32 & 0.00 & 0.00 & 1.76 & 0.35 & 0.76 \\
\hline
\end{tabular}




\begin{tabular}{|c|c|c|c|c|c|c|c|c|c|c|c|c|c|}
\hline \multirow{2}{*}{$\begin{array}{l}\text { Banding } \\
\text { day }\end{array}$} & \multirow[b]{2}{*}{ Date } & & \multicolumn{10}{|c|}{ Net } & \multirow{2}{*}{$\begin{array}{c}\text { Total } \\
\text { (hours:minutes) }\end{array}$} \\
\hline & & & 1 & 2 & 3 & 4 & 5 & 6 & 7 & 8 & 9 & 10 & \\
\hline \multirow[b]{3}{*}{11} & \multirow[b]{3}{*}{ 4-16-13 } & \multirow{3}{*}{$\begin{array}{l}\text { Net-hours (hours:minutes) } \\
\text { Captures (number) } \\
\text { Captures per net-hour }\end{array}$} & $2: 50$ & $3: 00$ & 3:00 & 3:00 & $3: 00$ & $2: 50$ & $2: 50$ & $3: 10$ & $2: 50$ & $2: 40$ & 29:10 \\
\hline & & & 2 & 0 & 1 & 2 & 1 & 0 & 0 & 2 & 1 & 1 & 10 \\
\hline & & & 0.71 & 0.00 & 0.33 & 0.67 & 0.33 & 0.00 & 0.00 & 0.63 & 0.35 & 0.38 & 0.34 \\
\hline \multirow[b]{3}{*}{12} & \multirow[b]{3}{*}{ 4-17-13 } & \multirow{3}{*}{$\begin{array}{l}\text { Net-hours (hours:minutes) } \\
\text { Captures (number) } \\
\text { Captures per net-hour }\end{array}$} & $3: 10$ & $3: 10$ & $2: 50$ & $2: 50$ & 3:00 & $3: 00$ & $3: 00$ & $3: 00$ & 3:00 & $3: 10$ & $30: 10$ \\
\hline & & & 0 & 1 & 1 & 0 & 1 & 0 & 1 & 0 & 1 & 0 & 5 \\
\hline & & & 0.00 & 0.32 & 0.35 & 0.00 & 0.33 & 0.00 & 0.33 & 0.00 & 0.33 & 0.00 & 0.17 \\
\hline \multirow[b]{3}{*}{13} & \multirow[b]{3}{*}{ 4-18-13 } & \multirow{3}{*}{$\begin{array}{l}\text { Net-hours (hours:minutes) } \\
\text { Captures (number) } \\
\text { Captures per net-hour }\end{array}$} & $3: 10$ & $3: 30$ & $3: 10$ & $2: 50$ & $3: 20$ & $3: 10$ & $2: 50$ & $3: 00$ & $3: 00$ & $3: 10$ & $31: 10$ \\
\hline & & & 1 & 4 & 0 & 3 & 3 & 3 & 3 & 1 & 2 & 4 & $25^{1}$ \\
\hline & & & 0.32 & 1.14 & 0.00 & 1.06 & 0.90 & 0.95 & 1.06 & 0.33 & 0.67 & 1.26 & 0.80 \\
\hline \multirow[b]{3}{*}{14} & \multirow[b]{3}{*}{$4-19-13$} & \multirow{3}{*}{$\begin{array}{l}\text { Net-hours (hours:minutes) } \\
\text { Captures (number) } \\
\text { Captures per net-hour }\end{array}$} & $2: 50$ & $3: 10$ & $3: 20$ & 3:10 & $3: 30$ & $3: 20$ & $3: 00$ & $3: 20$ & $3: 10$ & $3: 00$ & $31: 50$ \\
\hline & & & 1 & 4 & 6 & 9 & 8 & 6 & 3 & 1 & 7 & 0 & 45 \\
\hline & & & 0.35 & 1.26 & 1.80 & 2.84 & 2.29 & 1.80 & 1.00 & 0.30 & 2.21 & 0.00 & 1.41 \\
\hline \multirow[b]{3}{*}{15} & \multirow[b]{3}{*}{$4-22-13$} & \multirow{3}{*}{$\begin{array}{l}\text { Net-hours (hours:minutes) } \\
\text { Captures (number) } \\
\text { Captures per net-hour }\end{array}$} & $3: 10$ & $3: 00$ & $2: 40$ & $3: 00$ & $3: 00$ & $3: 20$ & $3: 00$ & $3: 20$ & $3: 00$ & $3: 00$ & $30: 30$ \\
\hline & & & 3 & 1 & 0 & 0 & 1 & 0 & 3 & 2 & 2 & 2 & 14 \\
\hline & & & 0.95 & 0.33 & 0.00 & 0.00 & 0.33 & 0.00 & 1.00 & 0.60 & 0.67 & 0.67 & 0.46 \\
\hline \multirow[b]{3}{*}{16} & \multirow[b]{3}{*}{$4-23-13$} & \multirow{3}{*}{$\begin{array}{l}\text { Net-hours (hours:minutes) } \\
\text { Captures (number) } \\
\text { Captures per net-hour }\end{array}$} & $3: 10$ & $3: 10$ & $1: 00$ & $2: 50$ & $3: 20$ & $3: 20$ & $3: 00$ & $3: 10$ & $3: 00$ & $3: 10$ & 29:10 \\
\hline & & & 0 & 0 & 2 & 2 & 3 & 3 & 1 & 0 & 4 & 1 & 16 \\
\hline & & & 0.00 & 0.00 & 2.00 & 0.71 & 0.90 & 0.90 & 0.33 & 0.00 & 1.33 & 0.32 & 0.55 \\
\hline \multirow[b]{3}{*}{17} & \multirow[b]{3}{*}{$4-24-13$} & \multirow{3}{*}{$\begin{array}{l}\text { Net-hours (hours:minutes) } \\
\text { Captures (number) } \\
\text { Captures per net-hour }\end{array}$} & $1: 00$ & $3: 00$ & $0: 00$ & $3: 00$ & 3:00 & $3: 00$ & $3: 00$ & $3: 00$ & $2: 50$ & $3: 00$ & $24: 50$ \\
\hline & & & 1 & 4 & 0 & 8 & 1 & 0 & 0 & 1 & 1 & 2 & 18 \\
\hline & & & 1.00 & 1.33 & 0.00 & 2.67 & 0.33 & 0.00 & 0.00 & 0.33 & 0.35 & 0.67 & 0.72 \\
\hline & & Net-hours (hours:minutes) & $3: 10$ & $3: 10$ & $0: 00$ & $3: 00$ & $3: 20$ & $3: 00$ & $3: 00$ & $3: 00$ & $3: 10$ & $3: 10$ & 28:00 \\
\hline & & Captures (number) & 0 & 2 & 0 & 3 & 1 & 0 & 2 & 0 & 5 & 1 & 14 \\
\hline 18 & $4-25-13$ & Captures per net-hour & 0.00 & 0.63 & 0.00 & 1.00 & 0.30 & 0.00 & 0.67 & 0.00 & 1.58 & 0.32 & 0.50 \\
\hline & & Net-hours (hours:minutes) & $3: 10$ & $3: 10$ & 0:00 & $3: 00$ & $3: 10$ & $3: 10$ & $3: 10$ & $3: 10$ & $3: 10$ & $3: 10$ & $28: 20$ \\
\hline & & Captures (number) & 0 & 0 & 0 & 0 & 1 & 1 & 1 & 0 & 3 & 4 & 10 \\
\hline 19 & $4-26-13$ & Captures per net-hour & 0.00 & 0.00 & 0.00 & 0.00 & 0.32 & 0.32 & 0.32 & 0.00 & 0.95 & 1.26 & 0.35 \\
\hline & & Net-hours (hours:minutes) & $5: 10$ & $5: 00$ & $5: 20$ & $5: 00$ & 5:00 & $5: 10$ & 5:00 & $5: 10$ & 5:00 & $0: 00$ & $45: 50$ \\
\hline & & Captures (number) & 0 & 0 & 0 & 0 & 1 & 0 & 0 & 1 & 0 & 0 & 2 \\
\hline 20 & $5-07-13$ & Captures per net-hour & 0.00 & 0.00 & 0.00 & 0.00 & 0.20 & 0.00 & 0.00 & 0.19 & 0.00 & 0.00 & 0.04 \\
\hline & & Net-hours (hours:minutes) & 5:00 & 5:10 & 5:10 & 5:10 & $5: 20$ & $5: 20$ & $4: 40$ & $5: 10$ & 5:00 & $5: 00$ & $51: 00$ \\
\hline & & Captures (number) & 7 & 3 & 6 & 4 & 2 & 1 & 0 & 2 & 1 & 4 & 30 \\
\hline 21 & $5-14-13$ & Captures per net-hour & 1.40 & 0.58 & 1.16 & 0.77 & 0.38 & 0.19 & 0.00 & 0.39 & 0.20 & 0.80 & 0.59 \\
\hline
\end{tabular}




\begin{tabular}{|c|c|c|c|c|c|c|c|c|c|c|c|c|c|}
\hline \multirow{2}{*}{$\begin{array}{c}\text { Banding } \\
\text { day }\end{array}$} & \multirow[b]{2}{*}{ Date } & & \multicolumn{10}{|c|}{ Net } & \multirow{2}{*}{$\begin{array}{c}\text { Total } \\
\text { (hours:minutes) }\end{array}$} \\
\hline & & & 1 & 2 & 3 & 4 & 5 & 6 & 7 & 8 & 9 & 10 & \\
\hline \multirow[b]{3}{*}{22} & \multirow[b]{3}{*}{$5-28-13$} & \multirow{3}{*}{$\begin{array}{l}\text { Net-hours (hours:minutes) } \\
\text { Captures (number) } \\
\text { Captures per net-hour }\end{array}$} & $5: 00$ & $5: 00$ & $5: 00$ & 5:00 & $5: 00$ & 5:00 & 5:00 & $5: 00$ & $5: 00$ & $5: 00$ & $50: 00$ \\
\hline & & & 4 & 0 & 1 & 0 & 1 & 0 & 0 & 0 & 1 & 0 & 7 \\
\hline & & & 0.80 & 0.00 & 0.20 & 0.00 & 0.20 & 0.00 & 0.00 & 0.00 & 0.20 & 0.00 & 0.14 \\
\hline \multirow{3}{*}{\multicolumn{2}{|c|}{$\begin{array}{c}\text { Totals by } \\
\text { net }\end{array}$}} & \multirow{3}{*}{$\begin{array}{l}\text { Net-hours (hours:minutes) } \\
\text { Captures (number) } \\
\text { Captures per net-hour }\end{array}$} & $71: 20$ & 75:00 & $62: 10$ & $71: 40$ & $74: 20$ & $73: 10$ & $72: 10$ & $74: 10$ & $69: 50$ & $66: 50$ & $710: 40$ \\
\hline & & & 27 & 34 & 33 & 45 & 35 & 25 & 21 & 12 & 45 & 24 & $304^{1}$ \\
\hline & & & 0.38 & 0.45 & 0.53 & 0.63 & 0.47 & 0.34 & 0.29 & 0.16 & 0.64 & 0.36 & 0.43 \\
\hline
\end{tabular}

${ }^{1}$ Total adjusted for captures with unknown net numbers. 
Table B5. Migrant capture rate by net and date during spring migration, Point Loma banding station, San Diego, California, 2014.

\begin{tabular}{|c|c|c|c|c|c|c|c|c|c|c|c|c|c|}
\hline \multirow{2}{*}{$\begin{array}{l}\text { Banding } \\
\text { day }\end{array}$} & \multirow[b]{2}{*}{ Date } & & \multicolumn{10}{|c|}{ Net } & \multirow{2}{*}{$\begin{array}{c}\text { Total } \\
\text { (hours:minutes) }\end{array}$} \\
\hline & & & 1 & 2 & 3 & 4 & 5 & 6 & 7 & 8 & 9 & 10 & \\
\hline \multirow[b]{3}{*}{1} & \multirow[b]{3}{*}{$4-1-14$} & \multirow{3}{*}{$\begin{array}{l}\text { Net-hours (hours:minutes) } \\
\text { Captures (number) } \\
\text { Captures per net-hour }\end{array}$} & $2: 50$ & $2: 50$ & $2: 50$ & $1: 20$ & $2: 50$ & $3: 00$ & $2: 10$ & $3: 10$ & $2: 50$ & $2: 50$ & $26: 40$ \\
\hline & & & 1 & 0 & 0 & 0 & 0 & 0 & 0 & 0 & 2 & 2 & 5 \\
\hline & & & 0.35 & 0.00 & 0.00 & 0.00 & 0.00 & 0.00 & 0.00 & 0.00 & 0.71 & 0.00 & 0.19 \\
\hline \multirow[b]{3}{*}{2} & \multirow[b]{3}{*}{$4-2-14$} & \multirow{3}{*}{$\begin{array}{l}\text { Net-hours (hours:minutes) } \\
\text { Captures (number) } \\
\text { Captures per net-hour }\end{array}$} & $3: 00$ & $3: 00$ & $2: 50$ & $0: 30$ & $3: 10$ & $3: 00$ & $0: 20$ & $1: 30$ & $3: 00$ & $2: 50$ & $23: 10$ \\
\hline & & & 0 & 0 & 0 & 0 & 0 & 2 & 0 & 1 & 0 & 0 & 3 \\
\hline & & & 0.00 & 0.00 & 0.00 & 0.00 & 0.00 & 0.67 & 0.00 & 0.67 & 0.00 & 0.00 & 0.13 \\
\hline \multirow[b]{3}{*}{3} & \multirow[b]{3}{*}{$4-3-14$} & \multirow{3}{*}{$\begin{array}{l}\text { Net-hours (hours:minutes) } \\
\text { Captures (number) } \\
\text { Captures per net-hour }\end{array}$} & $3: 10$ & $3: 00$ & $2: 40$ & $3: 00$ & $3: 10$ & $2: 50$ & $2: 50$ & $3: 00$ & $3: 10$ & $3: 10$ & $30: 00$ \\
\hline & & & 1 & 1 & 0 & 1 & 0 & 0 & 0 & 1 & 0 & 1 & 5 \\
\hline & & & 0.32 & 0.33 & 0.00 & 0.33 & 0.00 & 0.00 & 0.00 & 0.33 & 0.00 & 0.32 & 0.17 \\
\hline \multirow[b]{3}{*}{4} & \multirow[b]{3}{*}{$4-4-14$} & \multirow{3}{*}{$\begin{array}{l}\text { Net-hours (hours:minutes) } \\
\text { Captures (number) } \\
\text { Captures per net-hour }\end{array}$} & $3: 10$ & $3: 10$ & $3: 10$ & $3: 00$ & $3: 10$ & $3: 10$ & $3: 00$ & $3: 10$ & $3: 20$ & $3: 10$ & $31: 30$ \\
\hline & & & 1 & 0 & 0 & 0 & 0 & 0 & 1 & 1 & 5 & 0 & 8 \\
\hline & & & 0.32 & 0.00 & 0.00 & 0.00 & 0.00 & 0.00 & 0.33 & 0.32 & 1.50 & 0.00 & 0.25 \\
\hline \multirow[b]{3}{*}{5} & \multirow[b]{3}{*}{$4-7-14$} & \multirow{3}{*}{$\begin{array}{l}\text { Net-hours (hours:minutes) } \\
\text { Captures (number) } \\
\text { Captures per net-hour }\end{array}$} & $3: 10$ & 3:00 & $2: 50$ & $3: 00$ & $3: 10$ & 3:00 & $3: 00$ & $3: 10$ & $3: 10$ & $3: 10$ & $30: 40$ \\
\hline & & & 0 & 1 & 1 & 3 & 4 & 2 & 0 & 1 & 0 & 0 & 12 \\
\hline & & & 0.00 & 0.33 & 0.35 & 1.00 & 1.26 & 0.67 & 0.00 & 0.32 & 0.00 & 0.00 & 1.73 \\
\hline \multirow[b]{3}{*}{6} & \multirow[b]{3}{*}{$4-8-14$} & \multirow{3}{*}{$\begin{array}{l}\text { Net-hours (hours:minutes) } \\
\text { Captures (number) } \\
\text { Captures per net-hour }\end{array}$} & $3: 00$ & $3: 00$ & $3: 00$ & $3: 00$ & $3: 10$ & $3: 10$ & $3: 00$ & $3: 10$ & $2: 50$ & $3: 00$ & $30: 20$ \\
\hline & & & 0 & 4 & 0 & 3 & 2 & 3 & 0 & 1 & 2 & 1 & 16 \\
\hline & & & 0.00 & 1.33 & 0.00 & 1.00 & 0.63 & 0.95 & 0.00 & 0.32 & 0.71 & 0.33 & 0.53 \\
\hline \multirow[b]{3}{*}{7} & \multirow[b]{3}{*}{$4-9-14$} & \multirow{3}{*}{$\begin{array}{l}\text { Net-hours (hours:minutes) } \\
\text { Captures (number) } \\
\text { Captures per net-hour }\end{array}$} & $3: 00$ & $3: 00$ & $3: 00$ & $3: 00$ & $3: 00$ & $3: 00$ & $3: 00$ & $3: 00$ & $3: 00$ & $3: 00$ & $30: 00$ \\
\hline & & & 6 & 1 & 2 & 6 & 5 & 0 & 1 & 0 & 1 & 1 & 23 \\
\hline & & & 2.00 & 0.33 & 0.67 & 2.00 & 1.67 & 0.00 & 0.33 & 0.00 & 0.33 & 0.33 & 0.77 \\
\hline \multirow[b]{3}{*}{8} & & Net-hours (hours:minutes) & $3: 10$ & $3: 10$ & $3: 10$ & $3: 20$ & $3: 20$ & $3: 20$ & $3: 20$ & $3: 20$ & $3: 10$ & $3: 10$ & $32: 30$ \\
\hline & & Captures (number) & 1 & 2 & 1 & 2 & 2 & 2 & 0 & 4 & 3 & 4 & 21 \\
\hline & $4-10-14$ & Captures per net-hour & 0.32 & 0.63 & 0.32 & 0.60 & 0.60 & 0.60 & 0.00 & 1.20 & 0.95 & 1.26 & 0.65 \\
\hline & & Net-hours (hours:minutes) & $3: 10$ & $3: 10$ & $3: 00$ & $3: 20$ & $3: 10$ & $3: 20$ & $3: 10$ & $3: 10$ & $3: 10$ & $3: 10$ & $31: 50$ \\
\hline & & Captures (number) & 1 & 0 & 1 & 1 & 1 & 0 & 1 & 0 & 3 & 0 & 8 \\
\hline 9 & 4-11-14 & Captures per net-hour & 0.32 & 0.00 & 0.33 & 0.30 & 0.32 & 0.00 & 0.32 & 0.00 & 0.95 & 0.00 & 1.04 \\
\hline & & Net-hours (hours:minutes) & $3: 10$ & $3: 00$ & $3: 10$ & $3: 00$ & $2: 50$ & $3: 10$ & $3: 00$ & $3: 10$ & $3: 20$ & $3: 20$ & $31: 10$ \\
\hline & & Captures (number) & 4 & 0 & 4 & 1 & 6 & 5 & 0 & 2 & 2 & 0 & 24 \\
\hline 10 & $4-14-14$ & Captures per net hour & 1.23 & 0.00 & 1.26 & 0.33 & 2.12 & 1.58 & 0.00 & 0.63 & 0.60 & 0.00 & 0.77 \\
\hline
\end{tabular}




\begin{tabular}{|c|c|c|c|c|c|c|c|c|c|c|c|c|c|}
\hline \multirow{2}{*}{$\begin{array}{c}\text { Banding } \\
\text { day }\end{array}$} & \multirow[b]{2}{*}{ Date } & & \multicolumn{10}{|c|}{ Net } & \multirow{2}{*}{$\begin{array}{c}\text { Total } \\
\text { (hours:minutes) }\end{array}$} \\
\hline & & & 1 & 2 & 3 & 4 & 5 & 6 & 7 & 8 & 9 & 10 & \\
\hline \multirow[b]{3}{*}{11} & \multirow[b]{3}{*}{$4-15-14$} & \multirow{3}{*}{$\begin{array}{l}\text { Net-hours (hours:minutes) } \\
\text { Captures (number) } \\
\text { Captures per net-hour }\end{array}$} & $3: 10$ & $3: 10$ & $3: 10$ & $3: 10$ & $3: 10$ & $3: 10$ & $3: 10$ & $3: 10$ & $3: 10$ & $3: 10$ & $31: 40$ \\
\hline & & & 0 & 1 & 0 & 2 & 2 & 1 & 0 & 0 & 2 & 1 & 9 \\
\hline & & & 0.00 & 0.32 & 0.00 & 0.67 & 0.63 & 0.32 & 0.00 & 0.00 & 0.63 & 0.32 & 0.28 \\
\hline \multirow[b]{3}{*}{12} & \multirow[b]{3}{*}{ 4-16-14 } & \multirow{3}{*}{$\begin{array}{l}\text { Net-hours (hours:minutes) } \\
\text { Captures (number) } \\
\text { Captures per net-hour }\end{array}$} & $3: 20$ & $3: 00$ & $3: 00$ & $3: 00$ & $3: 10$ & $3: 10$ & $3: 00$ & $3: 10$ & $2: 50$ & $3: 10$ & $30: 50$ \\
\hline & & & 0 & 1 & 2 & 3 & 3 & 2 & 2 & 1 & 2 & 2 & 18 \\
\hline & & & 0.00 & 0.33 & 0.67 & 1.00 & 0.95 & 0.63 & 0.67 & 0.32 & 0.71 & 0.63 & 0.58 \\
\hline \multirow[b]{3}{*}{13} & \multirow[b]{3}{*}{$4-17-14$} & \multirow{3}{*}{$\begin{array}{l}\text { Net-hours (hours:minutes) } \\
\text { Captures (number) } \\
\text { Captures per net-hour }\end{array}$} & $3: 00$ & $3: 10$ & $3: 10$ & $3: 00$ & $3: 10$ & $3: 00$ & $3: 00$ & $3: 00$ & $3: 10$ & $3: 10$ & $30: 50$ \\
\hline & & & 1 & 0 & 1 & 2 & 1 & 0 & 1 & 1 & 0 & 0 & 7 \\
\hline & & & 0.33 & 0.00 & 0.32 & 0.67 & 0.32 & 0.00 & 0.33 & 0.33 & 0.00 & 0.00 & 0.23 \\
\hline \multirow[b]{3}{*}{14} & \multirow[b]{3}{*}{$4-18-14$} & \multirow{3}{*}{$\begin{array}{l}\text { Net-hours (hours:minutes) } \\
\text { Captures (number) } \\
\text { Captures per net-hour }\end{array}$} & $3: 00$ & $3: 00$ & $3: 00$ & $3: 00$ & $3: 00$ & 3:00 & 3:00 & 3:00 & 3:00 & 3:00 & $30: 00$ \\
\hline & & & 3 & 4 & 2 & 2 & 0 & 0 & 4 & 1 & 6 & 2 & 24 \\
\hline & & & 1.00 & 1.33 & 0.67 & 0.67 & 0.00 & 0.00 & 1.33 & 0.33 & 2.00 & 0.67 & 0.80 \\
\hline \multirow[b]{3}{*}{15} & \multirow[b]{3}{*}{$4-21-14$} & \multirow{3}{*}{$\begin{array}{l}\text { Net-hours (hours:minutes) } \\
\text { Captures (number) } \\
\text { Captures per net-hour }\end{array}$} & $3: 10$ & 3:00 & $3: 20$ & 3:00 & $3: 00$ & 3:00 & 3:00 & 3:00 & 3:00 & $2: 50$ & $30: 20$ \\
\hline & & & 1 & 0 & 4 & 2 & 1 & 0 & 1 & 2 & 5 & 0 & 16 \\
\hline & & & 0.32 & 0.00 & 1.20 & 0.67 & 0.33 & 0.00 & 0.33 & 0.67 & 1.67 & 0.00 & 1.75 \\
\hline \multirow[b]{3}{*}{16} & \multirow{3}{*}{\multicolumn{2}{|c|}{$\begin{array}{l}\text { Net-hours (hours:minutes) } \\
\text { Captures (number) } \\
\text { Captures per net-hour }\end{array}$}} & $3: 10$ & 3:00 & $3: 10$ & $3: 10$ & $3: 10$ & $3: 10$ & $3: 10$ & $3: 10$ & $3: 20$ & 3:00 & $31: 30$ \\
\hline & & & 1 & 3 & 3 & 3 & 2 & 0 & 0 & 1 & 6 & 0 & 19 \\
\hline & & & 0.32 & 1.00 & 0.95 & 0.95 & 0.63 & 0.00 & 0.00 & 0.32 & 1.80 & 0.00 & 0.60 \\
\hline \multirow[b]{3}{*}{17} & \multirow{3}{*}{\multicolumn{2}{|c|}{$\begin{array}{l}\text { Net-hours (hours:minutes) } \\
\text { Captures (number) } \\
\text { Captures per net-hour }\end{array}$}} & $3: 00$ & $3: 00$ & $3: 10$ & $3: 00$ & $3: 00$ & $3: 00$ & $3: 00$ & $3: 00$ & $3: 00$ & $2: 50$ & $30: 00$ \\
\hline & & & 0 & 0 & 1 & 1 & 1 & 1 & 0 & 0 & 2 & 0 & 6 \\
\hline & & & 0.00 & 0.00 & 0.32 & 0.33 & 0.33 & 0.33 & 0.00 & 0.00 & 0.67 & 0.00 & 0.20 \\
\hline \multirow[b]{3}{*}{18} & \multirow{3}{*}{\multicolumn{2}{|c|}{$\begin{array}{l}\text { Net-hours (hours:minutes) } \\
\text { Captures (number) } \\
\text { Captures per net-hour }\end{array}$}} & $3: 30$ & $3: 20$ & $3: 20$ & $3: 20$ & $3: 30$ & $3: 30$ & $3: 20$ & $3: 30$ & $3: 30$ & $3: 30$ & $34: 20$ \\
\hline & & & 1 & 0 & 1 & 3 & 1 & 1 & 1 & 2 & 2 & 0 & 12 \\
\hline & & & 0.29 & 0.00 & 0.30 & 0.90 & 0.29 & 0.29 & 0.30 & 0.57 & 0.57 & 0.00 & 0.35 \\
\hline \multirow[b]{3}{*}{19} & & & 3:00 & $3: 10$ & $3: 10$ & $3: 10$ & 3:00 & $3: 10$ & $3: 10$ & 3:00 & 3:00 & 3:00 & $30: 50$ \\
\hline & & Captures (number) & 0 & 0 & 0 & 0 & 0 & 0 & 1 & 0 & 2 & 0 & 3 \\
\hline & $4-25-14$ & Captures per net-hour & 0.00 & 0.00 & 0.00 & 0.00 & 0.00 & 0.00 & 0.32 & 0.00 & 0.67 & 0.00 & 1.07 \\
\hline & & Net-hours (hours:minutes) & $3: 00$ & $3: 10$ & $3: 10$ & $3: 20$ & $3: 20$ & $3: 20$ & $3: 30$ & $3: 50$ & 3:00 & $3: 00$ & $32: 40$ \\
\hline & & Captures (number) & 0 & 1 & 0 & 2 & 1 & 2 & 1 & 2 & 0 & 0 & 9 \\
\hline 20 & 4-28-14 & Captures per net-hour & 0.00 & 0.32 & 0.00 & 0.60 & 0.30 & 0.60 & 0.29 & 0.52 & 0.00 & 0.00 & 0.28 \\
\hline
\end{tabular}




\begin{tabular}{|c|c|c|c|c|c|c|c|c|c|c|c|c|c|}
\hline \multirow{2}{*}{$\begin{array}{c}\text { Banding } \\
\text { day }\end{array}$} & \multirow[b]{2}{*}{ Date } & & \multicolumn{10}{|c|}{ Net } & \multirow{2}{*}{$\begin{array}{c}\text { Total } \\
\text { (hours:minutes) }\end{array}$} \\
\hline & & & 1 & 2 & 3 & 4 & 5 & 6 & 7 & 8 & 9 & 10 & \\
\hline \multirow[b]{3}{*}{21} & \multirow[b]{3}{*}{$5-9-14$} & \multirow{3}{*}{$\begin{array}{l}\text { Net-hours (hours:minutes) } \\
\text { Captures (number) } \\
\text { Captures per net-hour }\end{array}$} & $5: 10$ & $5: 20$ & $5: 10$ & $5: 10$ & $5: 10$ & $5: 10$ & $5: 10$ & $5: 10$ & $5: 10$ & $5: 00$ & $51: 40$ \\
\hline & & & 10 & 2 & 1 & 5 & 4 & 4 & 4 & 10 & 3 & 4 & 47 \\
\hline & & & 1.94 & 0.38 & 0.19 & 0.97 & 0.77 & 0.77 & 0.77 & 1.94 & 0.58 & 0.80 & 0.64 \\
\hline \multirow[b]{3}{*}{22} & \multirow[b]{3}{*}{$5-15-14$} & \multirow{3}{*}{$\begin{array}{l}\text { Net-hours (hours:minutes) } \\
\text { Captures (number) } \\
\text { Captures per net-hour }\end{array}$} & $4: 40$ & $4: 20$ & $4: 30$ & $4: 20$ & $4: 10$ & $4: 30$ & $4: 20$ & $4: 20$ & $4: 20$ & $4: 20$ & $43: 50$ \\
\hline & & & 7 & 2 & 13 & 3 & 8 & 6 & 1 & 7 & 5 & 6 & 58 \\
\hline & & & 1.50 & 0.46 & 2.89 & 0.69 & 1.92 & 1.33 & 0.23 & 1.62 & 1.15 & 1.38 & 1.32 \\
\hline \multirow[b]{3}{*}{23} & \multirow[b]{3}{*}{$5-27-14$} & \multirow{3}{*}{$\begin{array}{l}\text { Net-hours (hours:minutes) } \\
\text { Captures (number) } \\
\text { Captures per net-hour }\end{array}$} & $5: 10$ & $5: 20$ & $5: 10$ & $5: 10$ & $5: 10$ & $5: 20$ & 5:00 & $5: 10$ & 5:00 & $5: 10$ & $51: 40$ \\
\hline & & & 6 & 8 & 4 & 17 & 4 & 6 & 5 & 4 & 7 & 8 & 69 \\
\hline & & & 1.16 & 1.50 & 0.77 & 3.29 & 0.77 & 1.13 & 1.00 & 0.77 & 1.40 & 1.55 & 1.34 \\
\hline \multirow{3}{*}{\multicolumn{2}{|c|}{$\begin{array}{c}\text { Totals by } \\
\text { net }\end{array}$}} & \multirow{3}{*}{$\begin{array}{l}\text { Net-hours (hours:minutes) } \\
\text { Captures (number) } \\
\text { Captures per net-hour }\end{array}$} & $77: 10$ & $76 ; 20$ & $76 ; 10$ & $72: 10$ & $77: 00$ & $77: 30$ & $72: 50$ & $76: 20$ & $76: 30$ & $76: 00$ & 758:00 \\
\hline & & & 45 & 31 & 41 & 62 & 48 & 37 & 24 & 42 & 60 & 32 & 422 \\
\hline & & & 0.58 & 0.41 & 0.54 & 0.86 & 0.62 & 0.48 & 0.33 & 0.55 & 0.78 & 0.42 & 0.56 \\
\hline
\end{tabular}


Table B6. Migrant capture rate by net and date during spring migration, Point Loma banding station, San Diego, California, 2015.

\begin{tabular}{|c|c|c|c|c|c|c|c|c|c|c|c|c|c|}
\hline \multirow{2}{*}{$\begin{array}{l}\text { Banding } \\
\text { day }\end{array}$} & \multirow[b]{2}{*}{ Date } & & \multicolumn{10}{|c|}{ Net } & \multirow{2}{*}{$\begin{array}{c}\text { Total } \\
\text { (hours:minutes) }\end{array}$} \\
\hline & & & 1 & 2 & 3 & 4 & 5 & 6 & 7 & 8 & 9 & 10 & \\
\hline \multirow[b]{3}{*}{1} & \multirow[b]{3}{*}{$4-1-15$} & \multirow{3}{*}{$\begin{array}{l}\text { Net-hours (hours:minutes) } \\
\text { Captures (number) } \\
\text { Captures per net-hour }\end{array}$} & $3: 20$ & $3: 10$ & $3: 30$ & $3: 30$ & $3: 50$ & $3: 10$ & $3: 30$ & 3:00 & $3: 20$ & $3: 00$ & $33: 20$ \\
\hline & & & 0 & 0 & 1 & 0 & 0 & 1 & 0 & 0 & 0 & 0 & 2 \\
\hline & & & 0.00 & 0.00 & 0.29 & 0.00 & 0.00 & 0.32 & 0.00 & 0.00 & 0.00 & 0.00 & 0.06 \\
\hline \multirow[b]{3}{*}{2} & \multirow[b]{3}{*}{ 4-2-15 } & \multirow{3}{*}{$\begin{array}{l}\text { Net-hours (hours:minutes) } \\
\text { Captures (number) } \\
\text { Captures per net-hour }\end{array}$} & $3: 00$ & $3: 00$ & $3: 00$ & $3: 00$ & $2: 50$ & $2: 50$ & $2: 50$ & $3: 00$ & $3: 00$ & $3: 10$ & $29: 40$ \\
\hline & & & 0 & 0 & 0 & 0 & 0 & 1 & 0 & 0 & 2 & 1 & 4 \\
\hline & & & 0.00 & 0.00 & 0.00 & 0.00 & 0.00 & 0.35 & 0.00 & 0.00 & 0.67 & 0.32 & 0.13 \\
\hline \multirow[b]{3}{*}{3} & \multirow[b]{3}{*}{ 4-3-15 } & \multirow{3}{*}{$\begin{array}{l}\text { Net-hours (hours:minutes) } \\
\text { Captures (number) } \\
\text { Captures per net-hour }\end{array}$} & 3:00 & 3:00 & 3:00 & 3:00 & $3: 20$ & $3: 10$ & 3:00 & $3: 20$ & $2: 50$ & 3:00 & $30: 40$ \\
\hline & & & 0 & 0 & 2 & 2 & 1 & 1 & 0 & 1 & 1 & 0 & 8 \\
\hline & & & 0.00 & 0.00 & 0.67 & 0.67 & 0.30 & 0.32 & 0.00 & 0.30 & 0.35 & 0.00 & 0.26 \\
\hline \multirow[b]{3}{*}{4} & \multirow[b]{3}{*}{ 4-6-15 } & \multirow{3}{*}{$\begin{array}{l}\text { Net-hours (hours:minutes) } \\
\text { Captures (number) } \\
\text { Captures per net-hour }\end{array}$} & $3: 10$ & $3: 10$ & $3: 10$ & 0:00 & 3:00 & 0:00 & $3: 10$ & $3: 10$ & 3:00 & $2: 50$ & $24: 40$ \\
\hline & & & 0 & 0 & 0 & 0 & 0 & 0 & 0 & 0 & 0 & 0 & 0 \\
\hline & & & 0.00 & 0.00 & 0.00 & 0.00 & 0.00 & 0.00 & 0.00 & 0.00 & 0.00 & 0.00 & 0.00 \\
\hline \multirow[b]{3}{*}{5} & \multirow[b]{3}{*}{$4-7-15$} & \multirow{3}{*}{$\begin{array}{l}\text { Net-hours (hours:minutes) } \\
\text { Captures (number) } \\
\text { Captures per net-hour }\end{array}$} & $3: 00$ & $3: 10$ & $3: 10$ & $3: 00$ & $3: 10$ & $3: 20$ & $3: 00$ & $3: 00$ & $3: 10$ & $3: 00$ & $31: 00$ \\
\hline & & & 0 & 0 & 0 & 0 & 1 & 0 & 0 & 0 & 0 & 0 & 1 \\
\hline & & & 0.00 & 0.00 & 0.00 & 0.00 & 0.32 & 0.00 & 0.00 & 0.00 & 0.00 & 0.00 & 1.71 \\
\hline \multirow[b]{3}{*}{6} & \multirow[b]{3}{*}{$4-8-15$} & \multirow{3}{*}{$\begin{array}{l}\text { Net-hours (hours:minutes) } \\
\text { Captures (number) } \\
\text { Captures per net-hour }\end{array}$} & 3:00 & $3: 20$ & $3: 20$ & $3: 20$ & $2: 50$ & $2: 50$ & 3:00 & $3: 10$ & 3:00 & 3:00 & $30: 50$ \\
\hline & & & 1 & 1 & 1 & 0 & 0 & 0 & 1 & 0 & 0 & 1 & 5 \\
\hline & & & 0.33 & 0.30 & 0.30 & 0.00 & 0.00 & 0.00 & 0.33 & 0.00 & 0.00 & 0.33 & 0.16 \\
\hline \multirow[b]{3}{*}{7} & \multirow[b]{3}{*}{ 4-9-15 } & \multirow{3}{*}{$\begin{array}{l}\text { Net-hours (hours:minutes) } \\
\text { Captures (number) } \\
\text { Captures per net-hour }\end{array}$} & $3: 10$ & 3:00 & 3:00 & $3: 00$ & $3: 10$ & 3:00 & 3:00 & 3:00 & $3: 10$ & $3: 10$ & $30: 40$ \\
\hline & & & 0 & 0 & 2 & 0 & 0 & 0 & 0 & 0 & 1 & 3 & 6 \\
\hline & & & 0.00 & 0.00 & 0.67 & 0.00 & 0.00 & 0.00 & 0.00 & 0.00 & 0.32 & 0.95 & 0.20 \\
\hline \multirow[b]{3}{*}{8} & & Net-hours (hours:minutes) & $3: 00$ & $3: 10$ & $2: 50$ & 3:00 & $3: 10$ & $3: 10$ & $3: 00$ & $3: 00$ & $3: 00$ & $3: 10$ & $30: 30$ \\
\hline & & Captures (number) & 2 & 0 & 3 & 1 & 0 & 1 & 0 & 0 & 2 & 2 & 11 \\
\hline & $4-10-15$ & Captures per net-hour & 0.67 & 0.00 & 1.06 & 0.33 & 0.00 & 0.32 & 0.00 & 0.00 & 0.67 & 0.63 & 0.36 \\
\hline & & Net-hours (hours:minutes) & $3: 00$ & $3: 10$ & $3: 20$ & $2: 50$ & $2: 50$ & $2: 50$ & 3:00 & $3: 00$ & $3: 00$ & $3: 20$ & $30: 20$ \\
\hline & & Captures (number) & 1 & 0 & 0 & 0 & 1 & 1 & 0 & 2 & 5 & 0 & 10 \\
\hline 9 & 4-13-15 & Captures per net-hour & 0.33 & 0.00 & 0.00 & 0.00 & 0.35 & 0.35 & 0.00 & 0.67 & 1.67 & 0.00 & 1.09 \\
\hline & & Net-hours (hours:minutes) & $3: 00$ & $3: 00$ & 3:00 & $3: 00$ & $3: 00$ & 3:00 & 3:00 & $3: 00$ & $3: 10$ & $3: 10$ & $30: 20$ \\
\hline & & Captures (number) & 2 & 2 & 5 & 5 & 0 & 0 & 1 & 4 & 2 & 3 & 24 \\
\hline 10 & $4-14-15$ & Captures per net-hour & 0.67 & 0.67 & 1.67 & 1.67 & 0.00 & 0.00 & 0.33 & 1.33 & 0.63 & 0.95 & 0.79 \\
\hline
\end{tabular}




\begin{tabular}{|c|c|c|c|c|c|c|c|c|c|c|c|c|c|}
\hline \multirow{2}{*}{$\begin{array}{c}\text { Banding } \\
\text { day }\end{array}$} & \multirow[b]{2}{*}{ Date } & & \multicolumn{10}{|c|}{ Net } & \multirow{2}{*}{$\begin{array}{c}\text { Total } \\
\text { (hours:minutes) }\end{array}$} \\
\hline & & & 1 & 2 & 3 & 4 & 5 & 6 & 7 & 8 & 9 & 10 & \\
\hline \multirow[b]{3}{*}{11} & \multirow[b]{3}{*}{ 4-15-15 } & \multirow{3}{*}{$\begin{array}{l}\text { Net-hours (hours:minutes) } \\
\text { Captures (number) } \\
\text { Captures per net-hour }\end{array}$} & $3: 10$ & $3: 00$ & $3: 00$ & $3: 10$ & $3: 10$ & $3: 20$ & $3: 10$ & $3: 10$ & $3: 10$ & $3: 20$ & $31: 40$ \\
\hline & & & 1 & 2 & 0 & 1 & 0 & 5 & 1 & 0 & 6 & 1 & 17 \\
\hline & & & 0.32 & 0.67 & 0.00 & 0.32 & 0.00 & 1.50 & 0.32 & 0.00 & 1.89 & 0.30 & 0.54 \\
\hline \multirow[b]{3}{*}{12} & \multirow[b]{3}{*}{ 4-16-15 } & \multirow{3}{*}{$\begin{array}{l}\text { Net-hours (hours:minutes) } \\
\text { Captures (number) } \\
\text { Captures per net-hour }\end{array}$} & 3:00 & $2: 50$ & $3: 00$ & 3:10 & $3: 10$ & $3: 10$ & $3: 20$ & $2: 50$ & 3:00 & 3:00 & $30: 30$ \\
\hline & & & 2 & 0 & 0 & 3 & 0 & 1 & 2 & 1 & 5 & 0 & 14 \\
\hline & & & 0.67 & 0.00 & 0.00 & 0.95 & 0.00 & 0.32 & 0.60 & 0.35 & 1.67 & 0.00 & 0.46 \\
\hline \multirow[b]{3}{*}{13} & \multirow[b]{3}{*}{ 4-17-15 } & \multirow{3}{*}{$\begin{array}{l}\text { Net-hours (hours:minutes) } \\
\text { Captures (number) } \\
\text { Captures per net-hour }\end{array}$} & $3: 10$ & $3: 10$ & $3: 10$ & $3: 20$ & $3: 00$ & $3: 10$ & $2: 50$ & $3: 00$ & $3: 10$ & $3: 10$ & $31: 10$ \\
\hline & & & 0 & 2 & 1 & 0 & 1 & 0 & 0 & 0 & 1 & 0 & 5 \\
\hline & & & 0.00 & 0.63 & 0.32 & 0.00 & 0.33 & 0.00 & 0.00 & 0.00 & 0.32 & 0.00 & 0.16 \\
\hline \multirow[b]{3}{*}{14} & \multirow[b]{3}{*}{ 4-20-15 } & \multirow{3}{*}{$\begin{array}{l}\text { Net-hours (hours:minutes) } \\
\text { Captures (number) } \\
\text { Captures per net-hour } \\
\end{array}$} & 3:00 & $3: 10$ & 3:00 & $2: 50$ & $2: 50$ & 3:00 & $3: 00$ & $3: 00$ & 3:00 & $3: 10$ & $30: 00$ \\
\hline & & & 0 & 4 & 2 & 3 & 1 & 3 & 3 & 3 & 5 & 3 & 27 \\
\hline & & & 0.00 & 1.26 & 0.67 & 1.06 & 0.35 & 1.00 & 1.00 & 1.00 & 1.67 & 0.95 & 0.90 \\
\hline \multirow[b]{3}{*}{15} & \multirow[b]{3}{*}{ 4-21-15 } & \multirow{3}{*}{$\begin{array}{l}\text { Net-hours (hours:minutes) } \\
\text { Captures (number) } \\
\text { Captures per net-hour }\end{array}$} & $3: 10$ & $3: 20$ & $3: 20$ & $3: 10$ & $3: 20$ & $3: 10$ & $3: 10$ & $3: 20$ & $3: 20$ & 3:00 & $32: 20$ \\
\hline & & & 1 & 0 & 1 & 6 & 1 & 2 & 2 & 0 & 8 & 5 & 26 \\
\hline & & & 0.32 & 0.00 & 0.30 & 1.89 & 0.30 & 0.63 & 0.63 & 0.00 & 2.40 & 1.67 & 1.64 \\
\hline \multirow[b]{3}{*}{16} & \multirow[b]{3}{*}{ 4-22-15 } & \multirow{3}{*}{$\begin{array}{l}\text { Net-hours (hours:minutes) } \\
\text { Captures (number) } \\
\text { Captures per net-hour }\end{array}$} & 3:00 & $3: 00$ & $3: 00$ & $3: 10$ & $3: 00$ & $3: 00$ & $3: 00$ & 3:00 & $3: 00$ & $2: 50$ & $30: 00$ \\
\hline & & & 3 & 1 & 2 & 1 & 0 & 0 & 0 & 0 & 5 & 3 & 15 \\
\hline & & & 1.00 & 0.33 & 0.67 & 0.32 & 0.00 & 0.00 & 0.00 & 0.00 & 1.67 & 1.06 & 0.50 \\
\hline \multirow[b]{3}{*}{17} & \multirow[b]{3}{*}{ 4-23-15 } & \multirow{3}{*}{$\begin{array}{l}\text { Net-hours (hours:minutes) } \\
\text { Captures (number) } \\
\text { Captures per net-hour }\end{array}$} & $3: 20$ & $3: 10$ & $3: 10$ & $3: 10$ & $3: 10$ & $3: 10$ & $3: 10$ & $3: 10$ & $3: 20$ & $3: 00$ & $31: 50$ \\
\hline & & & 2 & 1 & 0 & 4 & 0 & 0 & 0 & 1 & 6 & 3 & 17 \\
\hline & & & 0.60 & 0.32 & 0.00 & 1.26 & 0.00 & 0.00 & 0.00 & 0.32 & 1.80 & 1.00 & 0.53 \\
\hline \multirow[b]{3}{*}{18} & & Net-hours & 3:00 & $3: 00$ & 3:00 & $2: 50$ & $2: 50$ & $3: 00$ & $3: 10$ & $3: 00$ & 3:00 & $3: 00$ & $29: 50$ \\
\hline & & Captures & 2 & 1 & 0 & 2 & 1 & 0 & 0 & 0 & 2 & 0 & 8 \\
\hline & $4-24-15$ & Captures per net-hour & 0.67 & 0.33 & 0.00 & 0.71 & 0.35 & 0.00 & 0.00 & 0.00 & 0.67 & 0.00 & 0.27 \\
\hline & & Net-hours (hours:minutes) & $3: 10$ & $3: 10$ & $3: 10$ & $3: 10$ & $3: 00$ & $2: 50$ & 3:00 & $3: 10$ & $3: 00$ & $3: 20$ & $31: 00$ \\
\hline & & Captures (number) & 0 & 4 & 2 & 5 & 2 & 4 & 2 & 2 & 4 & 4 & 29 \\
\hline 19 & $4-27-15$ & Captures per net-hour & 0.00 & 1.26 & 0.63 & 1.58 & 0.67 & 1.41 & 0.67 & 0.63 & 1.33 & 1.20 & 1.06 \\
\hline & & Net-hours (hours:minutes) & $3: 00$ & $3: 10$ & $3: 10$ & $3: 10$ & $3: 00$ & $3: 00$ & $2: 50$ & 3:00 & $3: 10$ & $3: 20$ & $30: 50$ \\
\hline & & Captures (number) & 0 & 3 & 8 & 0 & 5 & 3 & 0 & 0 & 1 & 3 & 23 \\
\hline 20 & $4-28-15$ & Captures per net-hour & 0.00 & 0.95 & 2.53 & 0.00 & 1.67 & 1.00 & 0.00 & 0.00 & 0.32 & 0.90 & 0.75 \\
\hline
\end{tabular}




\begin{tabular}{|c|c|c|c|c|c|c|c|c|c|c|c|c|c|}
\hline \multirow{2}{*}{$\begin{array}{l}\text { Banding } \\
\text { day }\end{array}$} & \multirow[b]{2}{*}{ Date } & & \multicolumn{10}{|c|}{ Net } & \multirow{2}{*}{$\begin{array}{c}\text { Total } \\
\text { (hours:minutes) }\end{array}$} \\
\hline & & & 1 & 2 & 3 & 4 & 5 & 6 & 7 & 8 & 9 & 10 & \\
\hline \multirow[b]{3}{*}{21} & \multirow[b]{3}{*}{$5-5-15$} & \multirow{3}{*}{$\begin{array}{l}\text { Net-hours (hours:minutes) } \\
\text { Captures (number) } \\
\text { Captures per net-hour }\end{array}$} & $5: 00$ & 5:00 & $5: 00$ & 5:00 & $5: 00$ & $5: 00$ & $4: 10$ & $5: 00$ & $5: 00$ & $5: 00$ & $49: 10$ \\
\hline & & & 6 & 8 & 4 & 2 & 1 & 7 & 1 & 9 & 8 & 1 & 47 \\
\hline & & & 1.20 & 1.60 & 0.80 & 0.40 & 0.20 & 1.40 & 0.24 & 1.80 & 1.60 & 0.20 & 0.67 \\
\hline \multirow[b]{3}{*}{22} & \multirow[b]{3}{*}{ 5-12-15 } & \multirow{3}{*}{$\begin{array}{l}\text { Net-hours (hours:minutes) } \\
\text { Captures (number) } \\
\text { Captures per net-hour }\end{array}$} & $5: 30$ & $5: 10$ & $5: 10$ & $5: 00$ & $3: 00$ & $5: 00$ & $5: 10$ & $5: 10$ & $5: 10$ & $5: 10$ & $49: 30$ \\
\hline & & & 6 & 5 & 2 & 5 & 0 & 2 & 7 & 4 & 3 & 5 & 39 \\
\hline & & & 1.09 & 0.97 & 0.39 & 1.00 & 0.00 & 0.40 & 1.35 & 0.77 & 0.58 & 0.97 & 0.79 \\
\hline \multirow[b]{3}{*}{23} & \multirow[b]{3}{*}{$5-26-15$} & \multirow{3}{*}{$\begin{array}{l}\text { Net-hours (hours:minutes) } \\
\text { Captures (number) } \\
\text { Captures per net-hour }\end{array}$} & $5: 10$ & 5:00 & $5: 00$ & 5:00 & $4: 50$ & $5: 00$ & $5: 10$ & $4: 50$ & $5: 00$ & $5: 10$ & $50: 10$ \\
\hline & & & 4 & 2 & 0 & 4 & 4 & 4 & 0 & 1 & 4 & 8 & 31 \\
\hline & & & 0.77 & 0.40 & 0.00 & 0.80 & 0.83 & 0.80 & 0.00 & 0.21 & 0.80 & 1.55 & 0.62 \\
\hline \multirow{3}{*}{\multicolumn{2}{|c|}{$\begin{array}{l}\text { Totals by } \\
\text { net }\end{array}$}} & $\begin{array}{l}\text { Net-hours } \\
\text { (hours:minutes) }\end{array}$ & $77: 20$ & $77: 20$ & $77: 30$ & $73: 50$ & $74: 30$ & $73: 10$ & $75: 40$ & $76: 20$ & $77: 00$ & $77: 20$ & $760: 00$ \\
\hline & & Captures (number) & 33 & 36 & 36 & 44 & 19 & 36 & 20 & 28 & 71 & 46 & 369 \\
\hline & & Captures per net-hour & 0.43 & 0.47 & 0.46 & 0.60 & 0.26 & 0.49 & 0.26 & 0.37 & 0.92 & 0.59 & 0.49 \\
\hline
\end{tabular}


Table B7. Capture frequency of migrant individuals during spring migration, Point Loma banding station, San Diego, California, 2011.

[See appendix A for bird species codes]

\begin{tabular}{|c|c|c|c|c|c|}
\hline & \multicolumn{2}{|c|}{$\begin{array}{c}\text { Number of individuals per capture } \\
\text { incidence (banded birds only) }\end{array}$} & \multicolumn{3}{|c|}{ Total number of individuals } \\
\cline { 2 - 6 } $\begin{array}{c}\text { Species } \\
\text { code }\end{array}$ & $\mathbf{1}$ capture & 2 captures & $\begin{array}{c}\text { Banded } \\
\text { birds }\end{array}$ & $\begin{array}{c}\text { Unbanded } \\
\text { birds }\end{array}$ & All birds \\
\hline CAHU & 0 & 0 & 0 & 1 & 1 \\
\hline RUHU & 0 & 0 & 0 & 19 & 19 \\
\hline WEWP & 2 & 0 & 2 & 0 & 2 \\
\hline WIFL & 2 & 0 & 2 & 0 & 2 \\
\hline HAFL & 7 & 0 & 7 & 0 & 7 \\
\hline PSFL & 34 & 0 & 34 & 1 & 35 \\
\hline COFL & 1 & 0 & 1 & 0 & 1 \\
\hline WAVI & 45 & 1 & 46 & 0 & 46 \\
\hline HOWR & 1 & 0 & 1 & 0 & 1 \\
\hline RCKI & 2 & 0 & 2 & 0 & 2 \\
\hline BGGN & 1 & 0 & 1 & 0 & 1 \\
\hline SWTH & 10 & 0 & 10 & 0 & 10 \\
\hline HETH & 8 & 0 & 8 & 0 & 8 \\
\hline PHAI & 1 & 0 & 1 & 0 & 1 \\
\hline NAWA & 20 & 2 & 22 & 0 & 22 \\
\hline YEWA & 3 & 0 & 3 & 0 & 3 \\
\hline AUWA & 2 & 0 & 2 & 0 & 2 \\
\hline BTYW & 12 & 0 & 12 & 0 & 12 \\
\hline TOWA & 30 & 0 & 30 & 0 & 30 \\
\hline THWH & 3 & 0 & 3 & 0 & 3 \\
\hline HEWA & 12 & 0 & 12 & 0 & 12 \\
\hline MGWA & 2 & 0 & 2 & 0 & 2 \\
\hline WIWA & 88 & 1 & 89 & 0 & 89 \\
\hline YBCH & 1 & 0 & 1 & 0 & 1 \\
\hline WETA & 4 & 0 & 4 & 0 & 4 \\
\hline GTTO & 1 & 0 & 1 & 0 & 1 \\
\hline CHSP & 3 & 0 & 3 & 2 & 5 \\
\hline BCSP & 2 & 0 & 2 & 0 & 2 \\
\hline LISP & 3 & 0 & 3 & 1 & 4 \\
\hline WTSP & 1 & 0 & 1 & 0 & 1 \\
\hline WCSP & 33 & 0 & 34 & 2 & 36 \\
\hline GCSP & 9 & 0 & 0 & 10 \\
\hline BHGR & 20 & 0 & 20 & 401 \\
\hline BUOR & 6 & 0 & 0 & 0 \\
\hline Total & 369 & 0 & 0 & 0 \\
\hline
\end{tabular}


Table B8. Capture frequency of migrant individuals during spring migration, Point Loma banding station, San Diego, California, 2012.

[See appendix A for bird species codes]

\begin{tabular}{|c|c|c|c|c|c|}
\hline & \multicolumn{2}{|c|}{$\begin{array}{c}\text { Number of individuals per capture } \\
\text { incidence (banded birds only) }\end{array}$} & \multicolumn{2}{|c|}{ Total number of individuals } \\
\cline { 2 - 6 } $\begin{array}{c}\text { Species } \\
\text { code }\end{array}$ & 1 capture & 2 captures & $\begin{array}{c}\text { Banded } \\
\text { birds }\end{array}$ & $\begin{array}{c}\text { Unbanded } \\
\text { birds }\end{array}$ & All birds \\
\hline BCHU & 0 & 0 & 0 & 3 & 3 \\
\hline CAHU & 0 & 0 & 0 & 2 & 2 \\
\hline RUHU & 0 & 0 & 0 & 13 & 13 \\
\hline WEWP & 5 & 0 & 5 & 0 & 5 \\
\hline WIFL & 1 & 0 & 1 & 0 & 1 \\
\hline LEFL & 1 & 0 & 1 & 0 & 1 \\
\hline HAFL & 4 & 0 & 4 & 0 & 4 \\
\hline GRFL & 1 & 0 & 1 & 0 & 1 \\
\hline PSFL & 39 & 0 & 39 & 0 & 39 \\
\hline CAVI & 1 & 0 & 1 & 0 & 1 \\
\hline WAVI & 21 & 0 & 21 & 0 & 21 \\
\hline HOWR & 1 & 0 & 1 & 0 & 1 \\
\hline SWTH & 4 & 0 & 4 & 0 & 4 \\
\hline HETH & 9 & 6 & 15 & 0 & 15 \\
\hline NAWA & 7 & 0 & 7 & 0 & 7 \\
\hline YEWA & 1 & 0 & 1 & 0 & 1 \\
\hline AUWA & 4 & 0 & 4 & 0 & 4 \\
\hline BTYW & 3 & 0 & 3 & 0 & 3 \\
\hline TOWA & 9 & 0 & 9 & 0 & 9 \\
\hline HEWA & 9 & 0 & 9 & 0 & 9 \\
\hline MGWA & 2 & 0 & 2 & 0 & 2 \\
\hline COYE & 2 & 0 & 2 & 0 & 2 \\
\hline WIWA & 74 & 1 & 75 & 1 & 76 \\
\hline WETA & 2 & 0 & 2 & 0 & 2 \\
\hline CHSP & 1 & 0 & 1 & 0 & 1 \\
\hline SAVS & 0 & 0 & 0 & 1 & 1 \\
\hline FOSP & 1 & 0 & 1 & 0 & 1 \\
\hline LISP & 6 & 1 & 7 & 0 & 7 \\
\hline WCSP & 37 & 0 & 37 & 1 & 38 \\
\hline GCSP & 3 & 0 & 3 & 0 & 3 \\
\hline BHGR & 4 & 0 & 4 & 0 & 4 \\
\hline BLGR & 1 & 0 & 1 & 0 & 1 \\
\hline INBU & 1 & 0 & 1 & 0 & 1 \\
\hline BUOR & 3 & 0 & 21 & 286 \\
\hline Total & 257 & 0 & & 3 \\
\hline
\end{tabular}


Table B9. Capture frequency of migrant individuals during spring migration, Point Loma banding station, San Diego, California, 2013.

[See appendix A for bird species codes]

\begin{tabular}{|c|c|c|c|c|c|}
\hline & \multicolumn{2}{|c|}{$\begin{array}{c}\text { Number of individuals per capture } \\
\text { incidence (banded birds only) }\end{array}$} & \multicolumn{2}{|c|}{ Total number of individuals } \\
\cline { 2 - 6 } $\begin{array}{c}\text { Species } \\
\text { code }\end{array}$ & 1 capture & 2 captures & $\begin{array}{c}\text { Banded } \\
\text { birds }\end{array}$ & $\begin{array}{c}\text { Unbanded } \\
\text { birds }\end{array}$ & All birds \\
\hline SSHA & 0 & 0 & 0 & 1 & 1 \\
\hline BCHU & 0 & 0 & 0 & 4 & 4 \\
\hline CAHU & 0 & 0 & 0 & 2 & 2 \\
\hline RUHU & 0 & 0 & 0 & 15 & 15 \\
\hline WEWP & 2 & 0 & 2 & 0 & 2 \\
\hline HAFL & 5 & 0 & 5 & 0 & 5 \\
\hline GRFL & 2 & 0 & 2 & 0 & 2 \\
\hline PSFL & 36 & 0 & 36 & 0 & 36 \\
\hline COFL & 2 & 0 & 2 & 0 & 2 \\
\hline WEKI & 1 & 0 & 1 & 0 & 1 \\
\hline CAVI & 1 & 0 & 1 & 0 & 1 \\
\hline WAVI & 16 & 0 & 16 & 0 & 16 \\
\hline HOWR & 0 & 1 & 1 & 0 & 1 \\
\hline RCKI & 24 & 3 & 27 & 0 & 27 \\
\hline SWTH & 2 & 0 & 2 & 0 & 2 \\
\hline HETH & 10 & 0 & 10 & 0 & 10 \\
\hline NAWA & 8 & 0 & 8 & 0 & 8 \\
\hline AUWA & 5 & 0 & 5 & 0 & 5 \\
\hline BTYW & 5 & 0 & 5 & 0 & 5 \\
\hline TOWA & 8 & 0 & 8 & 0 & 8 \\
\hline HEWA & 4 & 0 & 4 & 0 & 4 \\
\hline MGWA & 7 & 0 & 7 & 0 & 7 \\
\hline COYE & 3 & 0 & 3 & 0 & 3 \\
\hline WIWA & 48 & 2 & 50 & 1 & 51 \\
\hline WETA & 5 & 0 & 5 & 0 & 5 \\
\hline CHSP & 1 & 0 & 1 & 0 & 1 \\
\hline SAVS & 1 & 0 & 1 & 0 & 1 \\
\hline FOSP & 2 & 0 & 2 & 0 & 2 \\
\hline LISP & 12 & 0 & 12 & 0 & 12 \\
\hline WCSP & 41 & 0 & 41 & 0 & 41 \\
\hline GCSP & 5 & 1 & 6 & 0 & 6 \\
\hline BHGR & 9 & 0 & 9 & 0 & 9 \\
\hline BUOR & 2 & 0 & 2 & 0 & 2 \\
\hline Total & 267 & 7 & 23 & 297 \\
\hline
\end{tabular}


Table B10. Capture frequency of migrant individuals during spring migration, Point Loma banding station, San Diego, California, 2014.

[See appendix A for bird species codes]

\begin{tabular}{|c|c|c|c|c|c|c|}
\hline \multirow[b]{2}{*}{$\begin{array}{l}\text { Species } \\
\text { code }\end{array}$} & \multicolumn{3}{|c|}{$\begin{array}{l}\text { Number of individuals per capture } \\
\text { incidence (banded birds only) }\end{array}$} & \multicolumn{3}{|c|}{ Total number of individuals } \\
\hline & 1 Capture & 2 Captures & 3 Captures & $\begin{array}{l}\text { Banded } \\
\text { birds }\end{array}$ & $\begin{array}{l}\text { Unbanded } \\
\text { birds }\end{array}$ & All birds \\
\hline $\mathrm{BCHU}$ & 0 & 0 & 0 & 0 & 1 & 1 \\
\hline CAHU & 0 & 0 & 0 & 0 & 13 & 13 \\
\hline RUHU & 0 & 0 & 0 & 0 & 30 & 30 \\
\hline DOWO & 2 & 0 & 0 & 2 & 0 & 2 \\
\hline WEWP & 1 & 0 & 0 & 1 & 0 & 1 \\
\hline WIFL & 1 & 0 & 0 & 1 & 0 & 1 \\
\hline HAFL & 8 & 0 & 0 & 8 & 0 & 8 \\
\hline PSFL & 98 & 0 & 0 & 98 & 0 & 98 \\
\hline COFL & 1 & 0 & 0 & 1 & 0 & 1 \\
\hline WEKI & 1 & 0 & 0 & 1 & 0 & 1 \\
\hline LBVI & 1 & 0 & 0 & 1 & 0 & 1 \\
\hline WAVI & 48 & 0 & 0 & 48 & 0 & 48 \\
\hline HOWR & 1 & 0 & 0 & 1 & 0 & 1 \\
\hline RCKI & 14 & 1 & 1 & 16 & 0 & 16 \\
\hline BGGN & 1 & 0 & 0 & 1 & 0 & 1 \\
\hline SWTH & 8 & 0 & 0 & 8 & 0 & 8 \\
\hline HETH & 9 & 3 & 0 & 12 & 0 & 12 \\
\hline NAWA & 5 & 0 & 0 & 5 & 0 & 5 \\
\hline YEWA & 5 & 0 & 0 & 5 & 0 & 5 \\
\hline AUWA & 2 & 0 & 0 & 2 & 0 & 2 \\
\hline BTYW & 5 & 0 & 0 & 5 & 0 & 5 \\
\hline TOWA & 23 & 1 & 0 & 24 & 1 & 25 \\
\hline THWH & 2 & 0 & 0 & 2 & 0 & 2 \\
\hline HEWA & 4 & 0 & 0 & 4 & 1 & 5 \\
\hline MGWA & 9 & 0 & 0 & 9 & 0 & 9 \\
\hline COYE & 4 & 0 & 0 & 4 & 0 & 4 \\
\hline WIWA & 61 & 2 & 0 & 63 & 3 & 66 \\
\hline WETA & 4 & 0 & 0 & 4 & 0 & 4 \\
\hline CHSP & 2 & 0 & 0 & 2 & 0 & 2 \\
\hline SAGS & 1 & 0 & 0 & 1 & 0 & 1 \\
\hline FOSP & 5 & 0 & 0 & 5 & 0 & 5 \\
\hline LISP & 1 & 0 & 0 & 1 & 0 & 1 \\
\hline WCSP & 20 & 0 & 0 & 20 & 0 & 20 \\
\hline GCSP & 3 & 1 & 0 & 4 & 0 & 4 \\
\hline ORJU & 1 & 0 & 0 & 1 & 0 & 1 \\
\hline BHGR & 3 & 0 & 0 & 3 & 0 & 3 \\
\hline Total & 354 & 8 & 1 & 363 & 49 & 412 \\
\hline
\end{tabular}


Table B11. Capture frequency of migrant individuals during spring migration, Point Loma banding station, San Diego, California, 2015.

[See appendix A for bird species codes]

\begin{tabular}{|c|c|c|c|c|c|}
\hline \multirow{2}{*}{$\begin{array}{l}\text { Species } \\
\text { code }\end{array}$} & \multicolumn{2}{|c|}{$\begin{array}{l}\text { Number of individuals per capture } \\
\text { incidence (banded birds only) }\end{array}$} & \multicolumn{3}{|c|}{ Total number of individuals } \\
\hline & 1 Capture & 2 Captures & Banded birds & Unbanded birds & All birds \\
\hline AMKE & 0 & 0 & 0 & 1 & 1 \\
\hline $\mathrm{BCHU}$ & 0 & 0 & 0 & 6 & 6 \\
\hline CAHU & 0 & 0 & 0 & 12 & 12 \\
\hline RUHU & 0 & 0 & 0 & 22 & 22 \\
\hline WEWP & 6 & 0 & 6 & 0 & 6 \\
\hline WIFL & 3 & 0 & 3 & 0 & 3 \\
\hline HAFL & 15 & 0 & 15 & 0 & 15 \\
\hline DUFL & 1 & 0 & 1 & 0 & 1 \\
\hline PSFL & 73 & 1 & 74 & 1 & 75 \\
\hline COFL & 5 & 0 & 5 & 0 & 5 \\
\hline SAPH & 2 & 0 & 2 & 0 & 2 \\
\hline CAVI & 1 & 0 & 1 & 0 & 1 \\
\hline WAVI & 45 & 1 & 46 & 0 & 46 \\
\hline RCKI & 4 & 1 & 5 & 0 & 5 \\
\hline SWTH & 2 & 0 & 2 & 0 & 2 \\
\hline HETH & 9 & 1 & 10 & 0 & 10 \\
\hline GRCA & 1 & 0 & 1 & 0 & 1 \\
\hline NAWA & 8 & 0 & 8 & 0 & 8 \\
\hline YEWA & 5 & 0 & 5 & 0 & 5 \\
\hline BTYW & 8 & 0 & 8 & 0 & 8 \\
\hline TOWA & 14 & 0 & 14 & 0 & 14 \\
\hline HEWA & 2 & 0 & 2 & 0 & 2 \\
\hline MGWA & 1 & 0 & 1 & 0 & 1 \\
\hline COYE & 1 & 0 & 1 & 0 & 1 \\
\hline WIWA & 81 & 0 & 81 & 1 & 82 \\
\hline CAWA & 1 & 0 & 1 & 0 & 1 \\
\hline WETA & 3 & 0 & 3 & 0 & 3 \\
\hline CHSP & 1 & 0 & 1 & 0 & 1 \\
\hline LISP & 4 & 0 & 4 & 0 & 4 \\
\hline WCSP & 10 & 1 & 11 & 0 & 11 \\
\hline GCSP & 4 & 0 & 4 & 0 & 4 \\
\hline BHGR & 5 & 0 & 5 & 0 & 5 \\
\hline BUOR & 1 & 0 & 1 & 0 & 1 \\
\hline Total & 316 & 5 & 321 & 43 & 364 \\
\hline
\end{tabular}


Table B12. Number of migrant bird captures by date during spring migration, Point Loma banding station, San Diego, California, 2011.

[See appendix A for bird species codes. Includes multiple captures of some individuals]

\begin{tabular}{|c|c|c|c|c|c|c|c|c|c|c|c|c|c|c|c|c|c|c|c|c|c|c|c|c|c|}
\hline \multirow[b]{4}{*}{ Species code } & \multicolumn{23}{|c|}{ Banding day } & & \multirow{4}{*}{$\begin{array}{c}\text { Captures } \\
\text { per 100 net } \\
\text { hours } \\
\text { (746:40:00 } \\
\text { total net } \\
\text { hours) } \\
\end{array}$} \\
\hline & 1 & 2 & 3 & 4 & 5 & 6 & 7 & 8 & 9 & 10 & 11 & 12 & 13 & 14 & 15 & 16 & 17 & 18 & 19 & 20 & 21 & 22 & 23 & & \\
\hline & \multicolumn{23}{|c|}{ Date } & & \\
\hline & 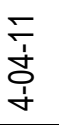 & 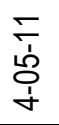 & 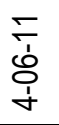 & 㝎 & 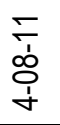 & $\frac{\underset{I}{\check{g}}}{\dot{T}}$ & $\underset{\dot{I}}{\stackrel{\check{g}}{\check{I}}}$ & $\underset{\frac{c}{\sigma}}{\stackrel{\leftarrow}{g}}$ & $\frac{\underset{J}{\dot{J}}}{\dot{g}}$ & 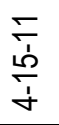 & 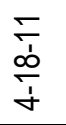 & $\frac{\bar{\sigma}}{\frac{\bar{g}}{\dot{g}}}$ & 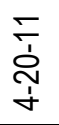 & $\frac{\underset{T}{\check{T}}}{\underset{f}{T}}$ & 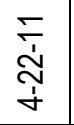 & 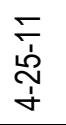 & $\begin{array}{l}\underset{\dot{c}}{\grave{S}} \\
\stackrel{+}{+}\end{array}$ & 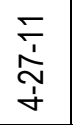 & 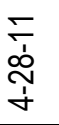 & 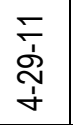 & 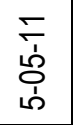 & 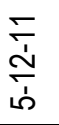 & 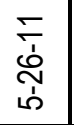 & Total & \\
\hline CAHU & 0 & 0 & 0 & 0 & 0 & 0 & 0 & 0 & 0 & 0 & 0 & 0 & 0 & 0 & 0 & 0 & 0 & 0 & 1 & 0 & 0 & 0 & 0 & 1 & 0.13 \\
\hline RUHU & 4 & 1 & 0 & 0 & 0 & 2 & 0 & 1 & 1 & 6 & 0 & 0 & 0 & 0 & 0 & 0 & 0 & 3 & 1 & 0 & 0 & 0 & 0 & 19 & 2.54 \\
\hline WEWP & 0 & 0 & 0 & 0 & 0 & 0 & 0 & 0 & 0 & 0 & 0 & 0 & 0 & 0 & 0 & 0 & 0 & 0 & 0 & 0 & 0 & 1 & 1 & 2 & 0.27 \\
\hline WIFL & 0 & 0 & 0 & 0 & 0 & 0 & 0 & 0 & 0 & 0 & 0 & 0 & 0 & 0 & 0 & 0 & 0 & 0 & 0 & 0 & 0 & 0 & 2 & 2 & 0.27 \\
\hline HAFL & 0 & 0 & 0 & 0 & 0 & 0 & 0 & 0 & 0 & 0 & 0 & 0 & 2 & 1 & 1 & 0 & 1 & 1 & 0 & 1 & 0 & 0 & 0 & 7 & 0.94 \\
\hline PSFL & 0 & 0 & 0 & 0 & 0 & 0 & 0 & 0 & 0 & 0 & 0 & 0 & 1 & 5 & 1 & 0 & 5 & 3 & 7 & 1 & 1 & 10 & 1 & 35 & 4.69 \\
\hline COFL & 0 & 0 & 0 & 0 & 0 & 0 & 0 & 0 & 0 & 0 & 0 & 0 & 0 & 0 & 0 & 0 & 0 & 0 & 0 & 0 & 0 & 0 & 1 & 1 & 0.13 \\
\hline WAVI & 0 & 0 & 1 & 0 & 0 & 0 & 0 & 1 & 0 & 0 & 0 & 0 & 2 & 4 & 4 & 1 & 5 & 4 & 4 & 1 & 2 & 8 & 10 & 47 & 6.29 \\
\hline HOWR & 0 & 0 & 0 & 0 & 0 & 1 & 0 & 0 & 0 & 0 & 0 & 0 & 0 & 0 & 0 & 0 & 0 & 0 & 0 & 0 & 0 & 0 & 0 & 1 & 0.13 \\
\hline RCKI & 0 & 0 & 0 & 0 & 1 & 1 & 0 & 0 & 0 & 0 & 0 & 0 & 0 & 0 & 0 & 0 & 0 & 0 & 0 & 0 & 0 & 0 & 0 & 2 & 0.27 \\
\hline BGGN & 0 & 0 & 1 & 0 & 0 & 0 & 0 & 0 & 0 & 0 & 0 & 0 & 0 & 0 & 0 & 0 & 0 & 0 & 0 & 0 & 0 & 0 & 0 & 1 & 0.13 \\
\hline SWTH & 0 & 0 & 0 & 0 & 0 & 0 & 0 & 0 & 0 & 0 & 0 & 0 & 0 & 0 & 1 & 0 & 1 & 0 & 0 & 0 & 2 & 6 & 0 & 10 & 1.34 \\
\hline HETH & 1 & 1 & 2 & 0 & 0 & 0 & 0 & 0 & 0 & 1 & 0 & 0 & 1 & 1 & 0 & 0 & 0 & 0 & 0 & 0 & 1 & 0 & 0 & 8 & 1.07 \\
\hline PHAl & 0 & 0 & 0 & 0 & 0 & 0 & 0 & 0 & 0 & 0 & 0 & 0 & 0 & 0 & 0 & 0 & 0 & 0 & 0 & 0 & 1 & 0 & 0 & 1 & 0.13 \\
\hline NAWA & 0 & 0 & 1 & 1 & 0 & 0 & 2 & 1 & 0 & 2 & 0 & 1 & 1 & 1 & 2 & 0 & 2 & 7 & 1 & 2 & 0 & 0 & 0 & 24 & 3.21 \\
\hline YEWA & 0 & 0 & 0 & 0 & 0 & 0 & 0 & 0 & 0 & 0 & 0 & 0 & 0 & 0 & 0 & 0 & 0 & 0 & 0 & 0 & 1 & 0 & 2 & 3 & 0.40 \\
\hline AUWA & 0 & 0 & 0 & 0 & 0 & 1 & 0 & 0 & 0 & 0 & 0 & 0 & 0 & 0 & 0 & 0 & 0 & 1 & 0 & 0 & 0 & 0 & 0 & 2 & 0.27 \\
\hline BTYW & 0 & 0 & 0 & 0 & 0 & 0 & 0 & 0 & 0 & 1 & 0 & 0 & 0 & 4 & 0 & 1 & 0 & 5 & 0 & 0 & 0 & 1 & 0 & 12 & 1.61 \\
\hline TOWA & 0 & 0 & 0 & 1 & 0 & 0 & 0 & 0 & 0 & 0 & 0 & 0 & 1 & 3 & 0 & 0 & 1 & 10 & 0 & 1 & 2 & 10 & 1 & 30 & 4.02 \\
\hline THWH & 0 & 0 & 0 & 0 & 0 & 0 & 0 & 0 & 0 & 0 & 0 & 0 & 0 & 0 & 0 & 0 & 1 & 0 & 0 & 0 & 1 & 1 & 0 & 3 & 0.40 \\
\hline HEWA & 0 & 0 & 0 & 0 & 0 & 0 & 0 & 0 & 0 & 0 & 0 & 0 & 0 & 1 & 0 & 0 & 0 & 8 & 1 & 0 & 0 & 2 & 0 & 12 & 1.61 \\
\hline MGWA & 0 & 0 & 0 & 0 & 0 & 0 & 0 & 0 & 0 & 0 & 0 & 0 & 0 & 0 & 0 & 0 & 0 & 1 & 0 & 0 & 0 & 0 & 1 & 2 & 0.27 \\
\hline WIWA & 0 & 1 & 1 & 0 & 0 & 1 & 1 & 1 & 1 & 0 & 0 & 0 & 1 & 7 & 2 & 1 & 4 & 16 & 7 & 4 & 11 & 24 & 7 & 90 & 12.05 \\
\hline $\mathrm{YBCH}$ & 0 & 0 & 0 & 0 & 0 & 0 & 0 & 0 & 0 & 0 & 0 & 0 & 0 & 0 & 0 & 0 & 0 & 0 & 0 & 0 & 1 & 0 & 0 & 1 & 0.13 \\
\hline WETA & 0 & 0 & 0 & 0 & 0 & 0 & 0 & 0 & 0 & 0 & 1 & 0 & 0 & 0 & 0 & 0 & 0 & 0 & 0 & 0 & 0 & 3 & 0 & 4 & 0.54 \\
\hline
\end{tabular}




\begin{tabular}{|c|c|c|c|c|c|c|c|c|c|c|c|c|c|c|c|c|c|c|c|c|c|c|c|c|c|}
\hline \multirow[b]{4}{*}{ Species code } & \multicolumn{23}{|c|}{ Banding day } & \multirow[b]{4}{*}{ Total } & \multirow{4}{*}{$\begin{array}{c}\text { Captures } \\
\text { per } 100 \text { net } \\
\text { hours } \\
\text { (746:40:00 } \\
\text { total net } \\
\text { hours) }\end{array}$} \\
\hline & 1 & 2 & 3 & 4 & 5 & 6 & 7 & 8 & 9 & 10 & 11 & 12 & 13 & 14 & 15 & 16 & 17 & 18 & 19 & 20 & 21 & 22 & 23 & & \\
\hline & \multicolumn{23}{|c|}{ Date } & & \\
\hline & 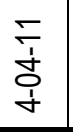 & 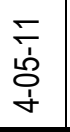 & $\begin{array}{l}\bar{c} \\
\bar{\phi} \\
\dot{1}\end{array}$ & 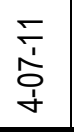 & 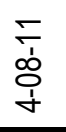 & 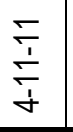 & $\underset{\grave{i}}{\stackrel{\check{g}}{\dot{t}}}$ & $\stackrel{\check{c}}{\stackrel{\check{m}}{\dot{g}}}$ & 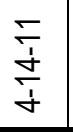 & $\frac{\check{c}}{\stackrel{5}{c}}$ & $\frac{\check{c}}{\stackrel{\check{c}}{c}}$ & $\frac{\bar{S}}{\stackrel{5}{\dot{T}}}$ & 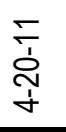 & 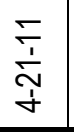 & 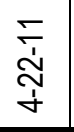 & 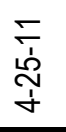 & 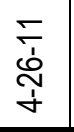 & 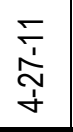 & 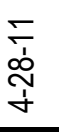 & $\begin{array}{l}\underset{+}{\grave{j}} \\
\stackrel{+}{+}\end{array}$ & 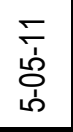 & 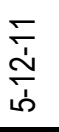 & 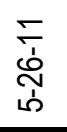 & & \\
\hline GTTO & 0 & 0 & 0 & 0 & 0 & 0 & 0 & 0 & 0 & 0 & 0 & 0 & 0 & 0 & 0 & 0 & 0 & 0 & 1 & 0 & 0 & 0 & 0 & 1 & 0.13 \\
\hline CHSP & 0 & 0 & 0 & 0 & 0 & 0 & 0 & 0 & 0 & 1 & 0 & 0 & 1 & 0 & 0 & 0 & 0 & 0 & 0 & 1 & 1 & 0 & 1 & 5 & 0.67 \\
\hline BCSP & 0 & 0 & 0 & 0 & 0 & 0 & 0 & 0 & 0 & 0 & 0 & 0 & 0 & 0 & 0 & 0 & 2 & 0 & 0 & 0 & 0 & 0 & 0 & 2 & 0.27 \\
\hline LISP & 0 & 0 & 1 & 0 & 0 & 2 & 0 & 0 & 0 & 0 & 0 & 0 & 0 & 0 & 0 & 0 & 0 & 0 & 1 & 0 & 0 & 0 & 0 & 4 & 0.54 \\
\hline WTSP & 0 & 0 & 0 & 0 & 0 & 0 & 0 & 0 & 0 & 0 & 0 & 0 & 0 & 0 & 0 & 0 & 0 & 0 & 0 & 0 & 1 & 0 & 0 & 1 & 0.13 \\
\hline WCSP & 1 & 0 & 1 & 0 & 0 & 3 & 0 & 2 & 0 & 5 & 0 & 0 & 8 & 7 & 1 & 3 & 0 & 4 & 2 & 0 & 0 & 0 & 0 & 37 & 4.95 \\
\hline GCSP & 1 & 0 & 1 & 0 & 0 & 0 & 0 & 0 & 1 & 2 & 1 & 0 & 1 & 0 & 3 & 0 & 0 & 0 & 1 & 0 & 0 & 0 & 0 & 11 & 1.47 \\
\hline BHGR & 1 & 0 & 0 & 0 & 0 & 1 & 1 & 0 & 0 & 0 & 0 & 0 & 0 & 4 & 1 & 2 & 8 & 2 & 0 & 0 & 0 & 0 & 0 & 20 & 2.68 \\
\hline BUOR & 0 & 0 & 0 & 0 & 0 & 0 & 0 & 0 & 0 & 1 & 0 & 0 & 0 & 1 & 1 & 0 & 1 & 1 & 0 & 0 & 0 & 1 & 0 & 6 & 0.80 \\
\hline Captures per day & 8 & 3 & 9 & 2 & 1 & 12 & 4 & 6 & 3 & 19 & 2 & 1 & 19 & 39 & 17 & 8 & 31 & 66 & 27 & 11 & 25 & 67 & 27 & 407 & 54.48 \\
\hline Total species & 5 & 3 & 8 & 2 & 1 & 8 & 3 & 5 & 3 & 8 & 2 & 1 & 9 & 11 & 9 & 5 & 10 & 13 & 11 & 6 & 11 & 11 & 10 & 32 & 4.28 \\
\hline
\end{tabular}


Table B13. Number of migrant bird captures by date during spring migration, Point Loma banding station, San Diego, California, 2012.

[See appendix A for bird species codes. Includes multiple captures of some individuals]

\begin{tabular}{|c|c|c|c|c|c|c|c|c|c|c|c|c|c|c|c|c|c|c|c|c|c|c|c|c|}
\hline \multirow[b]{4}{*}{ Species code } & \multicolumn{22}{|c|}{ Banding day } & & \multirow{4}{*}{$\begin{array}{c}\text { Captures } \\
\text { per } 100 \\
\text { net-hours } \\
\text { (714:50:00 } \\
\text { total net- } \\
\text { hours) }\end{array}$} \\
\hline & 1 & 2 & 3 & 4 & 5 & 6 & 7 & 8 & 9 & 10 & 11 & 12 & 13 & 14 & 15 & 16 & 17 & 18 & 19 & 20 & 21 & 22 & & \\
\hline & \multicolumn{22}{|c|}{ Date } & & \\
\hline & 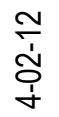 & 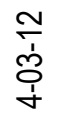 & $\underset{\stackrel{I}{+}}{\stackrel{+}{+}}$ & $\frac{\stackrel{N}{\dot{\delta}}}{\stackrel{+}{+}}$ & $\begin{array}{l}\stackrel{1}{\grave{d}} \\
\grave{+} \\
\dot{y}\end{array}$ & 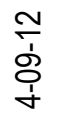 & $\frac{\stackrel{N}{\grave{c}}}{\dot{t}}$ & $\frac{\stackrel{N}{\check{T}}}{\dot{g}}$ & $\frac{\stackrel{N}{\grave{I}}}{\stackrel{+}{+}}$ & $\frac{\stackrel{N}{\grave{m}}}{\stackrel{\dot{q}}{*}}$ & $\frac{\stackrel{N}{\grave{d}}}{\frac{\dot{g}}{\dot{t}}}$ & $\frac{\stackrel{N}{\grave{I}}}{\frac{\dot{T}}{\dot{T}}}$ & $\frac{\stackrel{N}{o ́ ~}}{\frac{\dot{g}}{d}}$ & $\frac{\stackrel{N}{\grave{s}}}{\dot{+}}$ & $\begin{array}{l}\stackrel{\sim}{\grave{d}} \\
\text { ণ }\end{array}$ & 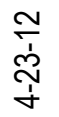 & $\underset{\stackrel{I}{+}}{\stackrel{\sim}{+}}$ & 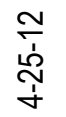 & 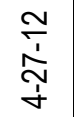 & $\begin{array}{l}\stackrel{̃}{0} \\
\text { ờ } \\
\text { ஸे }\end{array}$ & $\frac{\cong}{\stackrel{0}{\circ}}$ & 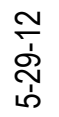 & Total & \\
\hline $\mathrm{BCHU}$ & 0 & 0 & 0 & 0 & 0 & 2 & 0 & 0 & 0 & 0 & 0 & 0 & 0 & 0 & 0 & 0 & 0 & 0 & 0 & 0 & 1 & 0 & 3 & 0.42 \\
\hline CAHU & 0 & 0 & 0 & 0 & 0 & 0 & 0 & 0 & 0 & 0 & 0 & 1 & 0 & 0 & 0 & 0 & 0 & 0 & 0 & 1 & 0 & 0 & 2 & 0.28 \\
\hline RUHU & 0 & 0 & 1 & 0 & 1 & 0 & 1 & 0 & 0 & 0 & 2 & 3 & 0 & 1 & 0 & 1 & 2 & 0 & 0 & 0 & 1 & 0 & 13 & 1.82 \\
\hline WEWP & 0 & 0 & 0 & 0 & 0 & 0 & 0 & 0 & 0 & 0 & 0 & 0 & 0 & 0 & 0 & 0 & 0 & 0 & 0 & 0 & 2 & 3 & 5 & 0.70 \\
\hline WIFL & 0 & 0 & 0 & 0 & 0 & 0 & 0 & 0 & 0 & 0 & 0 & 0 & 0 & 0 & 0 & 0 & 0 & 0 & 0 & 0 & 0 & 1 & 1 & 0.14 \\
\hline LEFL & 0 & 0 & 0 & 0 & 0 & 0 & 0 & 0 & 0 & 0 & 0 & 0 & 0 & 0 & 1 & 0 & 0 & 0 & 0 & 0 & 0 & 0 & 1 & 0.14 \\
\hline HAFL & 0 & 1 & 0 & 1 & 0 & 0 & 0 & 0 & 0 & 0 & 0 & 0 & 1 & 0 & 0 & 0 & 0 & 1 & 0 & 0 & 0 & 0 & 4 & 0.56 \\
\hline GRFL & 0 & 0 & 0 & 0 & 0 & 0 & 0 & 0 & 0 & 0 & 1 & 0 & 0 & 0 & 0 & 0 & 0 & 0 & 0 & 0 & 0 & 0 & 1 & 0.14 \\
\hline PSFL & 0 & 0 & 1 & 0 & 0 & 0 & 0 & 0 & 0 & 0 & 0 & 1 & 1 & 1 & 4 & 2 & 0 & 1 & 0 & 4 & 16 & 8 & 39 & 5.45 \\
\hline CAVI & 0 & 0 & 0 & 0 & 0 & 0 & 1 & 0 & 0 & 0 & 0 & 0 & 0 & 0 & 0 & 0 & 0 & 0 & 0 & 0 & 0 & 0 & 1 & 0.14 \\
\hline WAVI & 0 & 0 & 0 & 0 & 1 & 1 & 0 & 0 & 0 & 0 & 0 & 0 & 0 & 0 & 1 & 1 & 1 & 0 & 0 & 6 & 9 & 1 & 21 & 2.94 \\
\hline HOWR & 0 & 0 & 0 & 0 & 0 & 1 & 0 & 0 & 0 & 0 & 0 & 0 & 0 & 0 & 0 & 0 & 0 & 0 & 0 & 0 & 0 & 0 & 1 & 0.14 \\
\hline SWTH & 0 & 0 & 0 & 0 & 0 & 0 & 0 & 0 & 0 & 0 & 0 & 0 & 0 & 0 & 0 & 0 & 0 & 0 & 0 & 1 & 3 & 0 & 4 & 0.56 \\
\hline HETH & 5 & 4 & 2 & 1 & 1 & 0 & 4 & 0 & 3 & 0 & 0 & 1 & 0 & 0 & 0 & 0 & 0 & 0 & 0 & 0 & 0 & 0 & 21 & 2.94 \\
\hline NAWA & 0 & 0 & 0 & 0 & 0 & 0 & 0 & 0 & 0 & 0 & 0 & 2 & 1 & 0 & 3 & 0 & 1 & 0 & 0 & 0 & 0 & 0 & 7 & 0.98 \\
\hline YEWA & 0 & 0 & 0 & 0 & 0 & 0 & 0 & 0 & 0 & 0 & 0 & 0 & 0 & 0 & 0 & 0 & 1 & 0 & 0 & 0 & 0 & 0 & 1 & 0.14 \\
\hline AUWA & 0 & 0 & 0 & 1 & 0 & 0 & 1 & 0 & 0 & 0 & 0 & 0 & 0 & 0 & 1 & 0 & 0 & 0 & 0 & 1 & 0 & 0 & 4 & 0.56 \\
\hline BTYW & 0 & 0 & 0 & 0 & 0 & 1 & 0 & 0 & 0 & 0 & 1 & 0 & 0 & 0 & 0 & 0 & 0 & 0 & 1 & 0 & 0 & 0 & 3 & 0.42 \\
\hline TOWA & 0 & 0 & 0 & 0 & 0 & 0 & 0 & 0 & 0 & 0 & 0 & 0 & 0 & 0 & 0 & 1 & 0 & 1 & 0 & 6 & 1 & 0 & 9 & 1.26 \\
\hline HEWA & 0 & 0 & 0 & 0 & 0 & 0 & 0 & 0 & 0 & 0 & 0 & 0 & 1 & 1 & 0 & 3 & 0 & 0 & 0 & 4 & 0 & 0 & 9 & 1.26 \\
\hline MGWA & 0 & 0 & 0 & 0 & 0 & 0 & 0 & 0 & 0 & 0 & 0 & 0 & 1 & 0 & 0 & 1 & 0 & 0 & 0 & 0 & 0 & 0 & 2 & 0.28 \\
\hline COYE & 0 & 0 & 0 & 0 & 0 & 0 & 0 & 0 & 0 & 0 & 1 & 1 & 0 & 0 & 0 & 0 & 0 & 0 & 0 & 0 & 0 & 0 & 2 & 0.28 \\
\hline WIWA & 0 & 1 & 0 & 0 & 1 & 0 & 1 & 0 & 0 & 0 & 2 & 1 & 0 & 4 & 5 & 6 & 5 & 5 & 2 & 34 & 7 & 3 & 77 & 10.77 \\
\hline WETA & 0 & 0 & 0 & 0 & 0 & 0 & 0 & 0 & 0 & 0 & 0 & 0 & 0 & 0 & 1 & 0 & 0 & 0 & 0 & 0 & 1 & 0 & 2 & 0.28 \\
\hline CHSP & 0 & 0 & 0 & 0 & 0 & 0 & 0 & 0 & 0 & 0 & 0 & 0 & 0 & 0 & 0 & 1 & 0 & 0 & 0 & 0 & 0 & 0 & 1 & 0.14 \\
\hline
\end{tabular}




\begin{tabular}{|c|c|c|c|c|c|c|c|c|c|c|c|c|c|c|c|c|c|c|c|c|c|c|c|c|}
\hline \multirow[b]{4}{*}{ Species code } & \multicolumn{22}{|c|}{ Banding day } & & \multirow{4}{*}{$\begin{array}{c}\text { Captures } \\
\text { per } 100 \\
\text { net-hours } \\
\text { (714:50:00 } \\
\text { total net- } \\
\text { hours) }\end{array}$} \\
\hline & 1 & 2 & 3 & 4 & 5 & 6 & 7 & 8 & 9 & 10 & 11 & 12 & 13 & 14 & 15 & 16 & 17 & 18 & 19 & 20 & 21 & 22 & & \\
\hline & \multicolumn{22}{|c|}{ Date } & & \\
\hline & 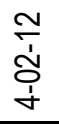 & $\begin{array}{c}\stackrel{1}{1} \\
\text { ơ⿱ } \\
\dot{t}\end{array}$ & $\begin{array}{l}\stackrel{N}{\dot{d}} \\
\stackrel{+}{+}\end{array}$ & $\begin{array}{l}\stackrel{\sim}{\grave{d}} \\
\stackrel{+}{+}\end{array}$ & 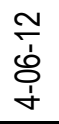 & 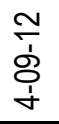 & $\frac{\stackrel{N}{\grave{d}}}{\dot{t}}$ & $\frac{\stackrel{ }{\grave{I}}}{\dot{f}}$ & 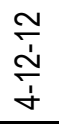 & 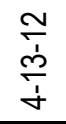 & 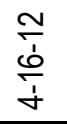 & $\frac{\stackrel{N}{\grave{I}}}{\frac{f}{d}}$ & 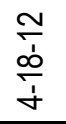 & $\frac{\stackrel{T}{T}}{\frac{S}{d}}$ & $\begin{array}{l}\stackrel{\sim}{\grave{d}} \\
\stackrel{+}{+}\end{array}$ & $\underset{\stackrel{N}{\tilde{I}}}{\stackrel{\sim}{+}}$ & $\underset{\stackrel{\sim}{+}}{\stackrel{\sim}{+}}$ & $\underset{\stackrel{N}{N}}{\stackrel{\sim}{+}}$ & 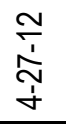 & $\begin{array}{l}\widetilde{N} \\
\text { ò } \\
\grave{1}\end{array}$ & 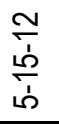 & 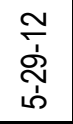 & Total & \\
\hline SAVS & 0 & 0 & 0 & 0 & 0 & 0 & 0 & 0 & 0 & 0 & 1 & 0 & 0 & 0 & 0 & 0 & 0 & 0 & 0 & 0 & 0 & 0 & 1 & 0.14 \\
\hline FOSP & 0 & 0 & 0 & 0 & 0 & 0 & 0 & 0 & 0 & 0 & 0 & 0 & 0 & 0 & 1 & 0 & 0 & 0 & 0 & 0 & 0 & 0 & 1 & 0.14 \\
\hline LISP & 0 & 1 & 0 & 1 & 0 & 0 & 0 & 0 & 0 & 2 & 1 & 3 & 0 & 0 & 0 & 0 & 0 & 0 & 0 & 0 & 0 & 0 & 8 & 1.12 \\
\hline WCSP & 1 & 0 & 4 & 0 & 3 & 4 & 3 & 0 & 0 & 0 & 8 & 11 & 1 & 0 & 2 & 1 & 0 & 0 & 0 & 0 & 0 & 0 & 38 & 5.31 \\
\hline GCSP & 0 & 0 & 0 & 1 & 1 & 0 & 0 & 0 & 0 & 0 & 0 & 0 & 0 & 0 & 0 & 0 & 1 & 0 & 0 & 0 & 0 & 0 & 3 & 0.42 \\
\hline BHGR & 0 & 1 & 0 & 0 & 0 & 0 & 0 & 0 & 0 & 0 & 0 & 3 & 0 & 0 & 0 & 0 & 0 & 0 & 0 & 0 & 0 & 0 & 4 & 0.56 \\
\hline BLGR & 0 & 0 & 0 & 0 & 0 & 0 & 0 & 0 & 0 & 0 & 0 & 0 & 0 & 1 & 0 & 0 & 0 & 0 & 0 & 0 & 0 & 0 & 1 & 0.14 \\
\hline INBU & 0 & 0 & 0 & 0 & 0 & 0 & 0 & 0 & 0 & 0 & 0 & 0 & 0 & 0 & 0 & 0 & 0 & 0 & 0 & 0 & 0 & 1 & 1 & 0.14 \\
\hline BUOR & 0 & 0 & 0 & 0 & 0 & 0 & 0 & 0 & 0 & 0 & 1 & 1 & 0 & 0 & 0 & 1 & 0 & 0 & 0 & 0 & 0 & 0 & 3 & 0.42 \\
\hline Captures per day & 6 & 8 & 8 & 5 & 8 & 9 & 11 & 0 & 3 & 2 & 18 & 28 & 6 & 8 & 19 & 18 & 11 & 8 & 3 & 57 & 41 & 17 & 294 & 41.12 \\
\hline Total species & 4 & 7 & 6 & 7 & 8 & 7 & 8 & 0 & 3 & 3 & 11 & 13 & 8 & 7 & 10 & 12 & 8 & 6 & 4 & 10 & 11 & 8 & 33 & 4.62 \\
\hline
\end{tabular}


Table B14. Number of migrant bird captures by date during spring migration, Point Loma banding station, San Diego, California, 2013.

[See appendix A for bird species codes. Includes multiple captures of some individuals]

\begin{tabular}{|c|c|c|c|c|c|c|c|c|c|c|c|c|c|c|c|c|c|c|c|c|c|c|c|c|}
\hline \multirow[b]{4}{*}{ Species code } & \multicolumn{22}{|c|}{ Banding day } & & \multirow{4}{*}{$\begin{array}{c}\text { Captures } \\
\text { per } 100 \\
\text { net-hours } \\
(710: 40: 00 \\
\text { total net- } \\
\text { hours }\end{array}$} \\
\hline & 1 & 2 & 3 & 4 & 5 & 6 & 7 & 8 & 9 & 10 & 11 & 12 & 13 & 14 & 15 & 16 & 17 & 18 & 19 & 20 & 21 & 22 & & \\
\hline & \multicolumn{22}{|c|}{ Date } & & \\
\hline & 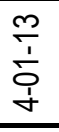 & 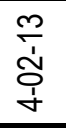 & $\begin{array}{l}\stackrel{m}{\grave{1}} \\
\dot{\delta} \\
\dot{y}\end{array}$ & $\begin{array}{l}\stackrel{m}{\dot{y}} \\
\dot{d} \\
\dot{q}\end{array}$ & 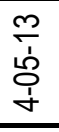 & $\begin{array}{l}m \\
\stackrel{m}{\dot{\delta}} \\
0 \\
\dot{q}\end{array}$ & $\begin{array}{l}\stackrel{m}{1} \\
\text { ơ } \\
\dot{+}\end{array}$ & $\frac{m}{\grave{c}}$ & $\frac{m}{\stackrel{m}{\dot{g}}}$ & $\frac{m}{\stackrel{m}{\grave{d}}}$ & $\begin{array}{l}m \\
\dot{c} \\
\dot{\sigma} \\
\dot{g}\end{array}$ & $\frac{m}{\stackrel{m}{\grave{r}}}$ & 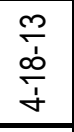 & $\frac{m}{\frac{m}{\dot{d}}}$ & $\begin{array}{l}\stackrel{m}{\grave{N}} \\
\stackrel{\text { I }}{+}\end{array}$ & 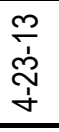 & $\underset{\substack{\mathfrak{I} \\
\stackrel{\mathfrak{I}}{+}}}{ }$ & $\begin{array}{l}\stackrel{m}{\grave{d}} \\
\stackrel{\leftrightarrow}{+}\end{array}$ & $\begin{array}{l}m \\
\stackrel{m}{\grave{d}} \\
\stackrel{+}{+}\end{array}$ & 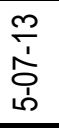 & $\frac{\stackrel{m}{\dot{J}}}{\stackrel{m}{\dot{b}}}$ & 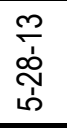 & Total & \\
\hline SSHA & 0 & 0 & 0 & 0 & 0 & 0 & 0 & 0 & 0 & 0 & 1 & 0 & 0 & 0 & 0 & 0 & 0 & 0 & 0 & 0 & 0 & 0 & 1 & 0.14 \\
\hline $\mathrm{BCHU}$ & 0 & 0 & 1 & 0 & 0 & 0 & 0 & 0 & 0 & 0 & 1 & 0 & 2 & 0 & 0 & 0 & 0 & 0 & 0 & 0 & 0 & 0 & 4 & 0.56 \\
\hline CAHU & 0 & 0 & 0 & 0 & 0 & 0 & 0 & 0 & 0 & 0 & 0 & 0 & 2 & 0 & 0 & 0 & 0 & 0 & 0 & 0 & 0 & 0 & 2 & 0.28 \\
\hline RUHU & 1 & 0 & 2 & 0 & 0 & 0 & 0 & 1 & 2 & 0 & 0 & 1 & 1 & 3 & 0 & 0 & 0 & 2 & 2 & 0 & 0 & 0 & 15 & 2.11 \\
\hline WEWP & 0 & 0 & 0 & 0 & 0 & 0 & 0 & 0 & 0 & 0 & 0 & 0 & 0 & 0 & 0 & 0 & 0 & 0 & 0 & 0 & 2 & 0 & 2 & 0.28 \\
\hline HAFL & 0 & 0 & 1 & 1 & 0 & 0 & 0 & 0 & 0 & 1 & 0 & 0 & 0 & 1 & 0 & 0 & 0 & 0 & 0 & 0 & 1 & 0 & 5 & 0.70 \\
\hline GRFL & 0 & 0 & 0 & 0 & 0 & 0 & 0 & 0 & 0 & 0 & 0 & 0 & 0 & 0 & 0 & 0 & 1 & 0 & 0 & 0 & 1 & 0 & 2 & 0.28 \\
\hline PSFL & 0 & 0 & 2 & 6 & 1 & 0 & 0 & 0 & 1 & 2 & 2 & 0 & 0 & 0 & 3 & 6 & 1 & 2 & 1 & 0 & 3 & 6 & 36 & 5.07 \\
\hline COFL & 0 & 0 & 0 & 0 & 0 & 0 & 0 & 0 & 0 & 1 & 0 & 0 & 0 & 0 & 0 & 0 & 0 & 0 & 0 & 0 & 1 & 0 & 2 & 0.28 \\
\hline WEKI & 0 & 0 & 0 & 0 & 0 & 0 & 0 & 0 & 1 & 0 & 0 & 0 & 0 & 0 & 0 & 0 & 0 & 0 & 0 & 0 & 0 & 0 & 1 & 0.14 \\
\hline CAVI & 0 & 0 & 0 & 0 & 0 & 0 & 0 & 0 & 0 & 0 & 0 & 0 & 0 & 0 & 0 & 0 & 0 & 0 & 0 & 0 & 1 & 0 & 1 & 0.14 \\
\hline WAVI & 0 & 0 & 0 & 0 & 0 & 0 & 0 & 0 & 1 & 1 & 0 & 0 & 0 & 1 & 0 & 1 & 2 & 1 & 2 & 0 & 6 & 1 & 16 & 2.25 \\
\hline HOWR & 1 & 0 & 0 & 0 & 1 & 0 & 0 & 0 & 0 & 0 & 0 & 0 & 0 & 0 & 0 & 0 & 0 & 0 & 0 & 0 & 0 & 0 & 2 & 0.28 \\
\hline RCKI & 1 & 1 & 2 & 2 & 3 & 1 & 0 & 2 & 1 & 3 & 2 & 1 & 1 & 8 & 0 & 1 & 1 & 0 & 0 & 0 & 0 & 0 & 30 & 4.23 \\
\hline SWTH & 0 & 0 & 0 & 0 & 0 & 0 & 0 & 0 & 0 & 0 & 0 & 0 & 0 & 0 & 0 & 0 & 0 & 0 & 0 & 0 & 2 & 0 & 2 & 0.28 \\
\hline HETH & 2 & 1 & 0 & 0 & 3 & 0 & 0 & 0 & 0 & 1 & 1 & 0 & 0 & 1 & 0 & 0 & 0 & 1 & 0 & 0 & 0 & 0 & 10 & 1.41 \\
\hline NAWA & 0 & 0 & 1 & 0 & 0 & 0 & 0 & 2 & 1 & 2 & 0 & 0 & 2 & 0 & 0 & 0 & 0 & 0 & 0 & 0 & 0 & 0 & 8 & 1.13 \\
\hline AUWA & 0 & 0 & 0 & 0 & 1 & 1 & 0 & 1 & 0 & 0 & 0 & 0 & 1 & 1 & 0 & 0 & 0 & 0 & 0 & 0 & 0 & 0 & 5 & 0.70 \\
\hline BTYW & 0 & 0 & 0 & 0 & 0 & 2 & 0 & 1 & 0 & 1 & 0 & 0 & 0 & 0 & 0 & 0 & 1 & 0 & 0 & 0 & 0 & 0 & 5 & 0.70 \\
\hline TOWA & 0 & 0 & 0 & 0 & 0 & 0 & 0 & 0 & 0 & 0 & 0 & 0 & 1 & 1 & 1 & 1 & 3 & 0 & 0 & 1 & 0 & 0 & 8 & 1.13 \\
\hline HEWA & 0 & 0 & 0 & 0 & 0 & 0 & 0 & 0 & 0 & 0 & 0 & 0 & 0 & 1 & 0 & 0 & 1 & 0 & 0 & 0 & 2 & 0 & 4 & 0.56 \\
\hline MGWA & 0 & 0 & 0 & 0 & 0 & 0 & 0 & 0 & 0 & 0 & 0 & 0 & 1 & 3 & 1 & 0 & 0 & 0 & 1 & 0 & 1 & 0 & 7 & 0.99 \\
\hline COYE & 0 & 0 & 0 & 1 & 0 & 0 & 0 & 0 & 0 & 0 & 0 & 1 & 0 & 1 & 0 & 0 & 0 & 0 & 0 & 0 & 0 & 0 & 3 & 0.42 \\
\hline WIWA & 0 & 0 & 1 & 1 & 1 & 0 & 0 & 2 & 3 & 2 & 3 & 1 & 3 & 2 & 9 & 7 & 4 & 6 & 2 & 0 & 6 & 0 & 53 & 7.46 \\
\hline WETA & 0 & 0 & 0 & 0 & 0 & 0 & 0 & 0 & 0 & 0 & 0 & 0 & 0 & 0 & 0 & 0 & 1 & 0 & 1 & 0 & 3 & 0 & 5 & 0.70 \\
\hline
\end{tabular}




\begin{tabular}{|c|c|c|c|c|c|c|c|c|c|c|c|c|c|c|c|c|c|c|c|c|c|c|c|c|}
\hline \multirow[b]{4}{*}{ Species code } & \multicolumn{22}{|c|}{ Banding day } & \multirow[b]{4}{*}{ Total } & \multirow{4}{*}{$\begin{array}{c}\text { Captures } \\
\text { per } 100 \\
\text { net-hours } \\
(710: 40: 00 \\
\text { total net- } \\
\text { hours }\end{array}$} \\
\hline & 1 & 2 & 3 & 4 & 5 & 6 & 7 & 8 & 9 & 10 & 11 & 12 & 13 & 14 & 15 & 16 & 17 & 18 & 19 & 20 & 21 & 22 & & \\
\hline & \multicolumn{22}{|c|}{ Date } & & \\
\hline & $\frac{m}{\frac{m}{\grave{1}}}$ & 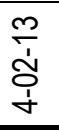 & $\begin{array}{l}\stackrel{m}{\grave{1}} \\
\dot{\delta} \\
\dot{+}\end{array}$ & $\begin{array}{l}\stackrel{m}{\dot{t}} \\
\stackrel{+}{+}\end{array}$ & 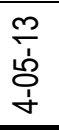 & 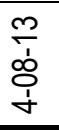 & 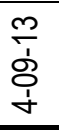 & $\frac{m}{\frac{m}{d}}$ & $\frac{\stackrel{m}{\frac{1}{t}}}{\dot{f}}$ & $\underset{\frac{m}{\grave{d}}}{\stackrel{m}{\dot{t}}}$ & $\begin{array}{l}\frac{m}{\grave{b}} \\
\frac{1}{d}\end{array}$ & 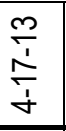 & $\begin{array}{l}\stackrel{m}{\infty} \\
\frac{0}{d} \\
\frac{d}{d}\end{array}$ & $\frac{m}{\frac{m}{d}}$ & $\begin{array}{l}\stackrel{m}{\grave{N}} \\
\stackrel{+}{+}\end{array}$ & $\begin{array}{l}\stackrel{m}{\grave{S}} \\
\stackrel{+}{+}\end{array}$ & 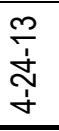 & $\begin{array}{l}\stackrel{m}{\dot{d}} \\
\stackrel{+}{+} \\
\end{array}$ & 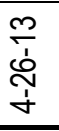 & 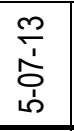 & $\frac{m}{\dot{d}}$ & $\begin{array}{l}\stackrel{m}{1} \\
\stackrel{0}{0} \\
i \\
i\end{array}$ & & \\
\hline CHSP & 0 & 0 & 0 & 0 & 0 & 0 & 0 & 0 & 0 & 0 & 0 & 0 & 0 & 0 & 0 & 0 & 1 & 0 & 0 & 0 & 0 & 0 & 1 & 0.14 \\
\hline SAVS & 0 & 0 & 0 & 0 & 0 & 0 & 0 & 0 & 0 & 0 & 0 & 0 & 0 & 1 & 0 & 0 & 0 & 0 & 0 & 0 & 0 & 0 & 1 & 0.14 \\
\hline FOSP & 0 & 1 & 0 & 1 & 0 & 0 & 0 & 0 & 0 & 0 & 0 & 0 & 0 & 0 & 0 & 0 & 0 & 0 & 0 & 0 & 0 & 0 & 2 & 0.28 \\
\hline LISP & 1 & 0 & 0 & 0 & 0 & 0 & 0 & 3 & 3 & 1 & 0 & 0 & 1 & 2 & 0 & 0 & 0 & 0 & 1 & 0 & 0 & 0 & 12 & 1.69 \\
\hline WCSP & 0 & 0 & 0 & 0 & 0 & 0 & 1 & 2 & 7 & 7 & 0 & 1 & 9 & 14 & 0 & 0 & 0 & 0 & 0 & 0 & 0 & 0 & 41 & 5.77 \\
\hline GCSP & 1 & 1 & 1 & 0 & 1 & 0 & 0 & 0 & 0 & 1 & 0 & 0 & 0 & 0 & 0 & 0 & 0 & 2 & 0 & 0 & 0 & 0 & 7 & 0.99 \\
\hline BHGR & 0 & 0 & 0 & 0 & 1 & 0 & 0 & 0 & 0 & 0 & 0 & 0 & 1 & 3 & 0 & 0 & 2 & 0 & 0 & 1 & 1 & 0 & 9 & 1.27 \\
\hline BUOR & 0 & 0 & 0 & 0 & 0 & 0 & 0 & 0 & 0 & 0 & 0 & 0 & 0 & 2 & 0 & 0 & 0 & 0 & 0 & 0 & 0 & 0 & 2 & 0.28 \\
\hline Captures per day & 7 & 4 & 11 & 12 & 12 & 4 & 1 & 14 & 20 & 23 & 10 & 5 & 25 & 45 & 14 & 16 & 18 & 14 & 10 & 2 & 30 & 7 & 304 & 42.82 \\
\hline Total species & 6 & 4 & 8 & 6 & 8 & 3 & 1 & 8 & 9 & 12 & 6 & 5 & 12 & 16 & 4 & 5 & 10 & 6 & 7 & 2 & 12 & 2 & 32 & 4.51 \\
\hline
\end{tabular}


Table B15. Number of migrant bird captures by date during spring migration, Point Loma banding station, San Diego, California, 2014.

[See appendix A for bird species codes. Includes multiple captures of some individuals]

\begin{tabular}{|c|c|c|c|c|c|c|c|c|c|c|c|c|c|c|c|c|c|c|c|c|c|c|c|c|c|}
\hline \multirow[b]{4}{*}{ Species code } & \multicolumn{23}{|c|}{ Banding day } & \multirow{4}{*}{\multicolumn{2}{|c|}{$\begin{array}{c}\text { Captures per } \\
100 \text { net-hours } \\
\text { (758:00:00 } \\
\text { total net- } \\
\text { hours) }\end{array}$}} \\
\hline & 1 & 2 & 3 & 4 & 5 & 6 & 7 & 8 & 9 & 10 & 11 & 12 & 13 & 14 & 15 & 16 & 17 & 18 & 19 & 20 & 21 & 22 & 23 & & \\
\hline & \multicolumn{23}{|c|}{ Date } & & \\
\hline & 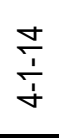 & $\underset{\stackrel{+}{+}}{\stackrel{+}{+}}$ & $\underset{\dot{d}}{\stackrel{+}{+}}$ & 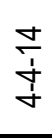 & 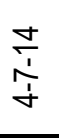 & $\begin{array}{l}\underset{+}{\Delta} \\
\stackrel{\leftrightarrow}{+}\end{array}$ & 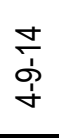 & $\frac{⿱ 亠 亠}{\grave{d}}$ & 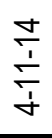 & $\frac{\vec{g}}{\stackrel{+}{\dot{d}}}$ & $\frac{\stackrel{+}{\dot{b}}}{\frac{\operatorname{s}}{\dot{g}}}$ & $\frac{\vec{J}}{\stackrel{\dot{\omega}}{\dot{t}}}$ & 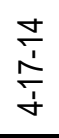 & $\frac{\vec{d}}{\stackrel{\dot{D}}{\dot{d}}}$ & $\underset{\frac{⿱ 亠 䒑}{\grave{I}}}{\stackrel{+}{+}}$ & 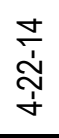 & 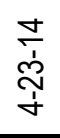 & 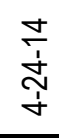 & $\underset{\substack{\stackrel{+}{N} \\
\stackrel{+}{+}}}{ }$ & 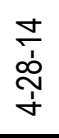 & 六 & $\frac{\vec{t}}{\frac{\dot{b}}{\dot{b}}}$ & 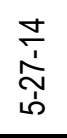 & & \\
\hline $\mathrm{BCHU}$ & 0 & 0 & 0 & 0 & 0 & 0 & 0 & 0 & 0 & 0 & 0 & 0 & 0 & 0 & 0 & 0 & 0 & 0 & 0 & 0 & 0 & 1 & 0 & 1 & 0.14 \\
\hline CAHU & 0 & 0 & 0 & 0 & 0 & 2 & 0 & 2 & 0 & 2 & 1 & 0 & 0 & 1 & 0 & 0 & 0 & 2 & 0 & 1 & 2 & 0 & 0 & 13 & 1.78 \\
\hline RUHU & 0 & 0 & 0 & 0 & 3 & 5 & 9 & 6 & 0 & 5 & 0 & 0 & 0 & 0 & 1 & 1 & 0 & 0 & 0 & 0 & 0 & 0 & 0 & 30 & 4.10 \\
\hline DOWO & 0 & 0 & 1 & 0 & 0 & 0 & 0 & 0 & 0 & 0 & 0 & 0 & 0 & 0 & 0 & 0 & 0 & 0 & 0 & 0 & 0 & 1 & 0 & 2 & 0.27 \\
\hline WEWP & 0 & 0 & 0 & 0 & 0 & 0 & 0 & 0 & 0 & 0 & 0 & 0 & 0 & 0 & 0 & 0 & 0 & 0 & 0 & 0 & 0 & 0 & 1 & 1 & 0.14 \\
\hline WIFL & 0 & 0 & 0 & 0 & 0 & 0 & 0 & 0 & 0 & 0 & 0 & 0 & 0 & 0 & 0 & 0 & 0 & 0 & 0 & 0 & 0 & 0 & 1 & 1 & 0.14 \\
\hline HAFL & 0 & 0 & 0 & 0 & 0 & 0 & 1 & 0 & 0 & 2 & 0 & 1 & 0 & 0 & 0 & 1 & 1 & 0 & 0 & 0 & 0 & 2 & 0 & 8 & 1.09 \\
\hline PSFL & 0 & 0 & 0 & 0 & 0 & 1 & 2 & 4 & 1 & 2 & 0 & 6 & 3 & 5 & 0 & 2 & 0 & 0 & 0 & 0 & 3 & 13 & 56 & 98 & 13.39 \\
\hline COFL & 0 & 0 & 0 & 0 & 0 & 1 & 0 & 0 & 0 & 0 & 0 & 0 & 0 & 0 & 0 & 0 & 0 & 0 & 0 & 0 & 0 & 0 & 0 & 1 & 0.14 \\
\hline WEKI & 0 & 0 & 0 & 0 & 0 & 0 & 0 & 1 & 0 & 0 & 0 & 0 & 0 & 0 & 0 & 0 & 0 & 0 & 0 & 0 & 0 & 0 & 0 & 1 & 0.14 \\
\hline LBVI & 0 & 0 & 0 & 0 & 0 & 0 & 0 & 0 & 0 & 0 & 0 & 0 & 0 & 0 & 0 & 0 & 0 & 0 & 0 & 0 & 0 & 0 & 1 & 1 & 0.14 \\
\hline WAVI & 0 & 1 & 0 & 0 & 1 & 0 & 0 & 2 & 0 & 1 & 0 & 0 & 0 & 2 & 1 & 2 & 2 & 3 & 0 & 3 & 21 & 2 & 7 & 48 & 6.56 \\
\hline HOWR & 0 & 0 & 0 & 0 & 0 & 0 & 0 & 1 & 0 & 0 & 0 & 0 & 0 & 0 & 0 & 0 & 0 & 0 & 0 & 0 & 0 & 0 & 0 & 1 & 0.14 \\
\hline RCKI & 2 & 0 & 1 & 2 & 2 & 1 & 3 & 1 & 0 & 1 & 3 & 0 & 0 & 0 & 0 & 2 & 0 & 0 & 1 & 0 & 0 & 0 & 0 & 19 & 2.60 \\
\hline BGGN & 0 & 1 & 0 & 0 & 0 & 0 & 0 & 0 & 0 & 0 & 0 & 0 & 0 & 0 & 0 & 0 & 0 & 0 & 0 & 0 & 0 & 0 & 0 & 1 & 0.14 \\
\hline SWTH & 0 & 0 & 0 & 0 & 0 & 0 & 0 & 0 & 0 & 0 & 0 & 0 & 0 & 0 & 0 & 0 & 0 & 0 & 0 & 0 & 3 & 2 & 3 & 8 & 1.09 \\
\hline HETH & 0 & 1 & 1 & 0 & 1 & 1 & 1 & 2 & 2 & 0 & 1 & 0 & 1 & 1 & 0 & 1 & 1 & 0 & 0 & 0 & 0 & 1 & 0 & 15 & 2.05 \\
\hline NAWA & 0 & 0 & 0 & 0 & 0 & 1 & 0 & 0 & 1 & 0 & 0 & 0 & 0 & 2 & 1 & 0 & 0 & 0 & 0 & 0 & 0 & 0 & 0 & 5 & 0.68 \\
\hline YEWA & 0 & 0 & 0 & 0 & 0 & 0 & 0 & 0 & 0 & 0 & 0 & 0 & 0 & 0 & 0 & 0 & 0 & 0 & 0 & 0 & 0 & 5 & 0 & 5 & 0.68 \\
\hline AUWA & 0 & 0 & 0 & 0 & 0 & 1 & 0 & 0 & 0 & 0 & 0 & 1 & 0 & 0 & 0 & 0 & 0 & 0 & 0 & 0 & 0 & 0 & 0 & 2 & 0.27 \\
\hline BTYW & 0 & 0 & 0 & 0 & 1 & 0 & 1 & 1 & 1 & 0 & 0 & 1 & 0 & 0 & 0 & 0 & 0 & 0 & 0 & 0 & 0 & 0 & 0 & 5 & 0.68 \\
\hline TOWA & 0 & 0 & 0 & 0 & 1 & 0 & 1 & 0 & 0 & 1 & 0 & 4 & 0 & 1 & 4 & 4 & 0 & 2 & 0 & 0 & 3 & 5 & 0 & 26 & 3.55 \\
\hline THWH & 0 & 0 & 0 & 0 & 0 & 0 & 0 & 0 & 0 & 0 & 0 & 0 & 0 & 0 & 2 & 0 & 0 & 0 & 0 & 0 & 0 & 0 & 0 & 2 & 0.27 \\
\hline HEWA & 0 & 0 & 0 & 0 & 0 & 0 & 0 & 0 & 0 & 1 & 0 & 0 & 0 & 0 & 0 & 0 & 0 & 0 & 0 & 1 & 2 & 1 & 0 & 5 & 0.68 \\
\hline MGWA & 0 & 0 & 0 & 0 & 0 & 0 & 0 & 0 & 0 & 1 & 0 & 0 & 0 & 0 & 0 & 1 & 0 & 0 & 0 & 0 & 0 & 7 & 0 & 9 & 1.23 \\
\hline
\end{tabular}




\begin{tabular}{|c|c|c|c|c|c|c|c|c|c|c|c|c|c|c|c|c|c|c|c|c|c|c|c|c|c|}
\hline \multirow[b]{4}{*}{ Species code } & \multicolumn{23}{|c|}{ Banding day } & \multirow[b]{4}{*}{ Total } & \multirow{4}{*}{$\begin{array}{c}\text { Captures per } \\
100 \text { net-hours } \\
\text { (758:00:00 } \\
\text { total net- } \\
\text { hours) }\end{array}$} \\
\hline & 1 & 2 & 3 & 4 & 5 & 6 & 7 & 8 & 9 & 10 & 11 & 12 & 13 & 14 & 15 & 16 & 17 & 18 & 19 & 20 & 21 & 22 & 23 & & \\
\hline & \multicolumn{23}{|c|}{ Date } & & \\
\hline & 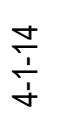 & 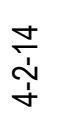 & 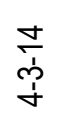 & 齐 & 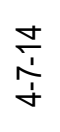 & 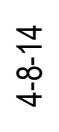 & 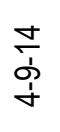 & $\frac{\dot{d}}{\frac{\dot{d}}{\dot{d}}}$ & 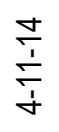 & $\frac{\vec{d}}{\dot{d}}$ & $\frac{\overrightarrow{\dot{b}}}{\frac{\dot{b}}{\dot{d}}}$ & $\frac{\vec{d}}{\dot{b}}$ & 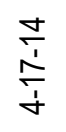 & $\frac{\vec{d}}{\frac{\dot{b}}{\dot{d}}}$ & 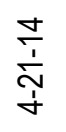 & 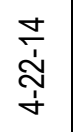 & 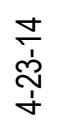 & 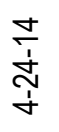 & 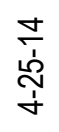 & 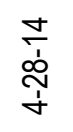 & 节 & $\frac{\vec{D}}{\stackrel{\vec{b}}{\dot{b}}}$ & 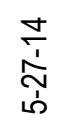 & & \\
\hline COYE & 0 & 0 & 0 & 1 & 0 & 1 & 0 & 0 & 1 & 0 & 0 & 0 & 0 & 0 & 0 & 0 & 1 & 0 & 0 & 0 & 0 & 0 & 0 & 4 & 0.55 \\
\hline WIWA & 0 & 0 & 1 & 1 & 0 & 0 & 1 & 0 & 0 & 2 & 2 & 5 & 2 & 10 & 5 & 4 & 0 & 3 & 2 & 3 & 12 & 15 & 0 & 68 & 9.29 \\
\hline WETA & 0 & 0 & 0 & 0 & 0 & 0 & 0 & 0 & 0 & 0 & 0 & 0 & 0 & 0 & 0 & 0 & 0 & 0 & 0 & 1 & 1 & 2 & 0 & 4 & 0.55 \\
\hline CHSP & 0 & 0 & 0 & 0 & 1 & 1 & 0 & 0 & 0 & 0 & 0 & 0 & 0 & 0 & 0 & 0 & 0 & 0 & 0 & 0 & 0 & 0 & 0 & 2 & 0.27 \\
\hline SAGS & 0 & 0 & 0 & 0 & 0 & 0 & 0 & 0 & 0 & 0 & 0 & 0 & 0 & 0 & 0 & 0 & 0 & 0 & 0 & 0 & 0 & 1 & 0 & 1 & 0.14 \\
\hline FOSP & 0 & 0 & 1 & 0 & 1 & 0 & 0 & 1 & 1 & 0 & 0 & 0 & 0 & 0 & 0 & 0 & 1 & 0 & 0 & 0 & 0 & 0 & 0 & 5 & 0.68 \\
\hline LISP & 0 & 0 & 0 & 0 & 0 & 0 & 0 & 0 & 0 & 0 & 1 & 0 & 0 & 0 & 0 & 0 & 0 & 0 & 0 & 0 & 0 & 0 & 0 & 1 & 0.14 \\
\hline WCSP & 2 & 0 & 0 & 3 & 0 & 1 & 4 & 0 & 1 & 5 & 1 & 0 & 1 & 1 & 0 & 1 & 0 & 0 & 0 & 0 & 0 & 0 & 0 & 20 & 2.73 \\
\hline GCSP & 1 & 0 & 0 & 0 & 1 & 0 & 0 & 0 & 0 & 0 & 0 & 0 & 0 & 1 & 2 & 0 & 0 & 0 & 0 & 0 & 0 & 0 & 0 & 5 & 0.68 \\
\hline ORJU & 0 & 0 & 0 & 0 & 0 & 0 & 0 & 0 & 0 & 1 & 0 & 0 & 0 & 0 & 0 & 0 & 0 & 0 & 0 & 0 & 0 & 0 & 0 & 1 & 0.14 \\
\hline BHGR & 0 & 0 & 0 & 1 & 0 & 0 & 0 & 0 & 0 & 0 & 0 & 0 & 0 & 0 & 0 & 0 & 0 & 2 & 0 & 0 & 0 & 0 & 0 & 3 & 0.41 \\
\hline Captures per day & 5 & 3 & 5 & 8 & 12 & 16 & 23 & 21 & 8 & 24 & 9 & 18 & 7 & 24 & 16 & 19 & 6 & 12 & 3 & 9 & 47 & 58 & 69 & 422 & 57.68 \\
\hline Total species & 3 & 3 & 5 & 5 & 9 & 11 & 9 & 10 & 7 & 12 & 6 & 6 & 4 & 9 & 7 & 10 & 5 & 5 & 2 & 5 & 8 & 14 & 6 & 36 & 4.92 \\
\hline
\end{tabular}


Table B16. Number of migrant bird captures by date during spring migration, Point Loma banding station, San Diego, California, 2015.

[See appendix A for bird species codes. Includes multiple captures of some individuals]

\begin{tabular}{|c|c|c|c|c|c|c|c|c|c|c|c|c|c|c|c|c|c|c|c|c|c|c|c|c|c|}
\hline \multirow[b]{4}{*}{ Species code } & \multicolumn{23}{|c|}{ Banding day } & & \multirow{4}{*}{$\begin{array}{c}\text { Captures } \\
\text { per } 100 \text { net- } \\
\text { hours } \\
\text { (760:00:00 } \\
\text { total net- } \\
\text { hours) }\end{array}$} \\
\hline & 1 & 2 & 3 & 4 & 5 & 6 & 7 & 8 & 9 & 10 & 11 & 12 & 13 & 14 & 15 & 16 & 17 & 18 & 19 & 20 & 21 & 22 & 23 & & \\
\hline & \multicolumn{23}{|c|}{ Date } & & \\
\hline & $\frac{\frac{6}{1}}{\dot{r}}$ & $\frac{\stackrel{0}{\dot{d}}}{\dot{\sim}}$ & $\frac{\stackrel{n}{\grave{r}}}{\stackrel{+}{+}}$ & $\begin{array}{l}\frac{0}{6} \\
\dot{\phi} \\
\dot{+}\end{array}$ & $\frac{\frac{10}{\dot{T}}}{\dot{j}}$ & 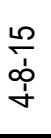 & $\frac{\stackrel{20}{s}}{\frac{s}{q}}$ & $\frac{\frac{10}{0}}{\frac{1}{q}}$ & $\frac{\frac{60}{\dot{m}}}{\frac{\dot{m}}{\dot{q}}}$ & $\frac{\stackrel{6}{\dot{d}}}{\dot{q}}$ & 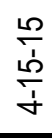 & 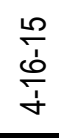 & 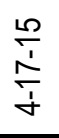 & $\frac{\stackrel{\circ}{\grave{d}}}{\underset{y}{+}}$ & 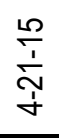 & 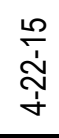 & $\frac{\stackrel{0}{\dot{m}}}{\stackrel{\sim}{+}}$ & 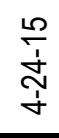 & 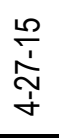 & 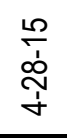 & 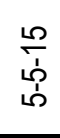 & 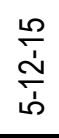 & 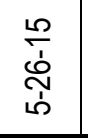 & Total & \\
\hline AMKE & 0 & 0 & 0 & 0 & 0 & 0 & 0 & 0 & 0 & 0 & 0 & 0 & 0 & 0 & 0 & 0 & 0 & 0 & 0 & 0 & 1 & 0 & 0 & 1 & 0.13 \\
\hline $\mathrm{BCHU}$ & 0 & 0 & 0 & 0 & 0 & 0 & 0 & 1 & 0 & 0 & 0 & 1 & 0 & 0 & 0 & 0 & 0 & 0 & 2 & 2 & 0 & 0 & 0 & 6 & 0.79 \\
\hline CAHU & 0 & 0 & 1 & 0 & 0 & 0 & 0 & 1 & 1 & 0 & 0 & 0 & 0 & 0 & 0 & 1 & 0 & 0 & 4 & 2 & 0 & 2 & 0 & 12 & 1.58 \\
\hline RUHU & 0 & 0 & 6 & 0 & 1 & 3 & 2 & 3 & 0 & 0 & 0 & 3 & 2 & 0 & 0 & 0 & 0 & 0 & 0 & 2 & 0 & 0 & 0 & 22 & 2.89 \\
\hline WEWP & 0 & 0 & 0 & 0 & 0 & 0 & 0 & 0 & 0 & 0 & 0 & 0 & 0 & 0 & 1 & 0 & 0 & 0 & 0 & 0 & 0 & 0 & 5 & 6 & 0.79 \\
\hline WIFL & 0 & 0 & 0 & 0 & 0 & 0 & 0 & 0 & 0 & 0 & 0 & 0 & 0 & 0 & 0 & 0 & 0 & 0 & 0 & 0 & 0 & 0 & 3 & 3 & 0.39 \\
\hline HAFL & 0 & 0 & 0 & 0 & 0 & 0 & 0 & 0 & 0 & 0 & 2 & 1 & 0 & 6 & 3 & 1 & 0 & 0 & 0 & 1 & 0 & 1 & 0 & 15 & 1.97 \\
\hline DUFL & 0 & 0 & 0 & 0 & 0 & 0 & 0 & 0 & 0 & 0 & 0 & 0 & 0 & 0 & 1 & 0 & 0 & 0 & 0 & 0 & 0 & 0 & 0 & 1 & 0.13 \\
\hline PSFL & 0 & 0 & 0 & 0 & 0 & 0 & 0 & 1 & 0 & 13 & 6 & 0 & 0 & 7 & 8 & 4 & 2 & 0 & 3 & 1 & 5 & 9 & 17 & 76 & 10.00 \\
\hline COFL & 0 & 0 & 0 & 0 & 0 & 0 & 0 & 1 & 0 & 0 & 0 & 0 & 0 & 2 & 0 & 0 & 1 & 0 & 1 & 0 & 0 & 0 & 0 & 5 & 0.66 \\
\hline SAPH & 0 & 0 & 0 & 0 & 0 & 0 & 0 & 0 & 0 & 0 & 0 & 0 & 0 & 0 & 0 & 0 & 0 & 0 & 0 & 2 & 0 & 0 & 0 & 2 & 0.26 \\
\hline CAVI & 0 & 0 & 0 & 0 & 0 & 0 & 0 & 0 & 0 & 0 & 0 & 0 & 0 & 0 & 0 & 0 & 1 & 0 & 0 & 0 & 0 & 0 & 0 & 1 & 0.13 \\
\hline WAVI & 0 & 0 & 0 & 0 & 0 & 0 & 0 & 0 & 0 & 1 & 1 & 2 & 0 & 6 & 0 & 1 & 4 & 1 & 4 & 1 & 16 & 9 & 1 & 47 & 6.18 \\
\hline RCKI & 0 & 0 & 0 & 0 & 0 & 0 & 0 & 0 & 0 & 0 & 1 & 0 & 1 & 0 & 1 & 1 & 1 & 0 & 0 & 0 & 1 & 0 & 0 & 6 & 0.79 \\
\hline SWTH & 0 & 0 & 0 & 0 & 0 & 0 & 0 & 0 & 0 & 0 & 0 & 0 & 0 & 1 & 0 & 0 & 0 & 0 & 0 & 1 & 0 & 0 & 0 & 2 & 0.26 \\
\hline HETH & 0 & 2 & 0 & 0 & 0 & 0 & 2 & 0 & 1 & 1 & 1 & 0 & 1 & 0 & 1 & 0 & 0 & 1 & 1 & 0 & 0 & 0 & 0 & 11 & 1.45 \\
\hline GRCA & 0 & 0 & 0 & 0 & 0 & 0 & 0 & 0 & 0 & 0 & 0 & 0 & 0 & 0 & 0 & 0 & 0 & 0 & 0 & 0 & 1 & 0 & 0 & 1 & 0.13 \\
\hline NAWA & 0 & 0 & 0 & 0 & 0 & 0 & 0 & 0 & 1 & 1 & 0 & 3 & 0 & 1 & 1 & 0 & 0 & 0 & 1 & 0 & 0 & 0 & 0 & 8 & 1.05 \\
\hline YEWA & 0 & 0 & 0 & 0 & 0 & 0 & 0 & 0 & 0 & 0 & 0 & 0 & 0 & 0 & 0 & 0 & 0 & 0 & 0 & 0 & 0 & 2 & 3 & 5 & 0.66 \\
\hline BTYW & 0 & 0 & 0 & 0 & 0 & 0 & 1 & 0 & 0 & 1 & 1 & 1 & 0 & 0 & 0 & 2 & 1 & 0 & 1 & 0 & 0 & 0 & 0 & 8 & 1.05 \\
\hline TOWA & 0 & 0 & 0 & 0 & 0 & 0 & 0 & 1 & 0 & 0 & 0 & 2 & 0 & 0 & 0 & 1 & 2 & 0 & 3 & 0 & 3 & 2 & 0 & 14 & 1.84 \\
\hline HEWA & 0 & 0 & 0 & 0 & 0 & 0 & 0 & 0 & 0 & 0 & 0 & 0 & 0 & 0 & 0 & 0 & 1 & 0 & 0 & 0 & 0 & 1 & 0 & 2 & 0.26 \\
\hline MGWA & 0 & 0 & 0 & 0 & 0 & 0 & 0 & 0 & 0 & 0 & 0 & 0 & 1 & 0 & 0 & 0 & 0 & 0 & 0 & 0 & 0 & 0 & 0 & 1 & 0.13 \\
\hline COYE & 0 & 0 & 0 & 0 & 0 & 0 & 0 & 0 & 0 & 0 & 0 & 0 & 0 & 0 & 1 & 0 & 0 & 0 & 0 & 0 & 0 & 0 & 0 & 1 & 0.13 \\
\hline WIWA & 0 & 0 & 0 & 0 & 0 & 0 & 0 & 1 & 0 & 6 & 3 & 0 & 0 & 3 & 8 & 4 & 4 & 6 & 7 & 9 & 20 & 10 & 1 & 82 & 10.79 \\
\hline
\end{tabular}




\begin{tabular}{|c|c|c|c|c|c|c|c|c|c|c|c|c|c|c|c|c|c|c|c|c|c|c|c|c|c|}
\hline \multirow[b]{4}{*}{ Species code } & \multicolumn{23}{|c|}{ Banding day } & \multirow[b]{4}{*}{ Total } & \multirow{4}{*}{$\begin{array}{c}\text { Captures } \\
\text { per } 100 \text { net- } \\
\text { hours } \\
\text { (760:00:00 } \\
\text { total net- } \\
\text { hours) }\end{array}$} \\
\hline & 1 & 2 & 3 & 4 & 5 & 6 & 7 & 8 & 9 & 10 & 11 & 12 & 13 & 14 & 15 & 16 & 17 & 18 & 19 & 20 & 21 & 22 & 23 & & \\
\hline & \multicolumn{23}{|c|}{ Date } & & \\
\hline & $\frac{\frac{10}{\frac{1}{d}}}{\frac{\dot{t}}{2}}$ & $\frac{\stackrel{20}{\dot{d}}}{\dot{\gamma}}$ & $\frac{\infty}{\dot{s}}$ & $\begin{array}{l}\frac{0}{\grave{o}} \\
\dot{+}\end{array}$ & $\frac{\stackrel{2}{\grave{1}}}{\dot{+}}$ & $\frac{n}{\grave{o}}$ & $\frac{\text { م) }}{\dot{q}}$ & 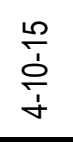 & $\frac{\stackrel{20}{\dot{m}}}{\stackrel{\dot{m}}{\dot{g}}}$ & 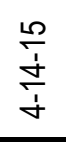 & 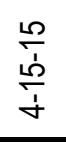 & $\frac{n}{\grave{c}}$ & $\frac{n}{\stackrel{1}{\grave{I}}}$ & 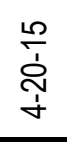 & $\frac{\stackrel{n}{\frac{1}{T}}}{\stackrel{+}{+}}$ & $\begin{array}{l}\underset{\dot{\sim}}{\grave{~}} \\
\stackrel{+}{+}\end{array}$ & 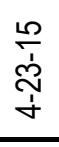 & 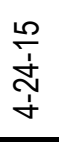 & 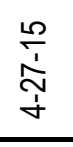 & $\begin{array}{l}\stackrel{0}{1} \\
\stackrel{1}{\mathfrak{d}} \\
\stackrel{+}{+}\end{array}$ & & $\frac{\stackrel{20}{\grave{\Lambda}}}{\stackrel{\dot{\omega}}{2}}$ & 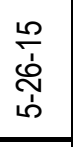 & & \\
\hline CAWA & 0 & 0 & 0 & 0 & 0 & 0 & 0 & 0 & 0 & 0 & 0 & 0 & 0 & 0 & 0 & 0 & 0 & 0 & 0 & 0 & 0 & 0 & 1 & 1 & 0.13 \\
\hline WETA & 0 & 0 & 0 & 0 & 0 & 0 & 0 & 0 & 0 & 0 & 0 & 0 & 0 & 0 & 0 & 0 & 0 & 0 & 0 & 0 & 0 & 3 & 0 & 3 & 0.39 \\
\hline CHSP & 0 & 1 & 0 & 0 & 0 & 0 & 0 & 0 & 0 & 0 & 0 & 0 & 0 & 0 & 0 & 0 & 0 & 0 & 0 & 0 & 0 & 0 & 0 & 1 & 0.13 \\
\hline LISP & 1 & 1 & 0 & 0 & 0 & 1 & 0 & 0 & 1 & 0 & 0 & 0 & 0 & 0 & 0 & 0 & 0 & 0 & 0 & 0 & 0 & 0 & 0 & 4 & 0.53 \\
\hline WCSP & 1 & 0 & 1 & 0 & 0 & 0 & 1 & 1 & 4 & 1 & 1 & 1 & 0 & 0 & 1 & 0 & 0 & 0 & 0 & 0 & 0 & 0 & 0 & 12 & 1.58 \\
\hline GCSP & 0 & 0 & 0 & 0 & 0 & 1 & 0 & 1 & 2 & 0 & 0 & 0 & 0 & 0 & 0 & 0 & 0 & 0 & 0 & 0 & 0 & 0 & 0 & 4 & 0.53 \\
\hline BHGR & 0 & 0 & 0 & 0 & 0 & 0 & 0 & 0 & 0 & 0 & 0 & 0 & 0 & 1 & 0 & 0 & 0 & 0 & 2 & 2 & 0 & 0 & 0 & 5 & 0.66 \\
\hline BUOR & 0 & 0 & 0 & 0 & 0 & 0 & 0 & 0 & 0 & 0 & 1 & 0 & 0 & 0 & 0 & 0 & 0 & 0 & 0 & 0 & 0 & 0 & 0 & 1 & 0.13 \\
\hline $\begin{array}{c}\text { Captures per } \\
\text { day }\end{array}$ & 2 & 4 & 8 & 0 & 1 & 5 & 6 & 11 & 10 & 24 & 17 & 14 & 5 & 27 & 26 & 15 & 17 & 8 & 29 & 23 & 47 & 39 & 31 & 369 & 48.55 \\
\hline Total species & 2 & 3 & 3 & 0 & 1 & 3 & 4 & 9 & 6 & 7 & 9 & 8 & 4 & 8 & 10 & 8 & 9 & 3 & 11 & 10 & 7 & 9 & 7 & 33 & 4.34 \\
\hline
\end{tabular}


Table B17. Sex and age of individual migrants (banded and unbanded) captured during spring migration, Point Loma banding station, San Diego, California, 2011.

[See appendix A for bird species codes. Age: AHY=after-hatching-year, SY=second-year, ASY=after-second-year, I=Indeterminable]

\begin{tabular}{|c|c|c|c|c|c|c|c|c|c|c|c|c|c|c|c|c|}
\hline \multirow{3}{*}{$\begin{array}{c}\text { Species } \\
\text { code }\end{array}$} & \multirow{2}{*}{\multicolumn{4}{|c|}{$\begin{array}{c}\text { Female } \\
\text { Age }\end{array}$}} & \multirow{3}{*}{$\begin{array}{c}\text { Female } \\
\text { total }\end{array}$} & \multirow{2}{*}{\multicolumn{4}{|c|}{$\begin{array}{l}\text { Male } \\
\text { Age } \\
\end{array}$}} & \multirow{3}{*}{$\begin{array}{l}\text { Male } \\
\text { total }\end{array}$} & \multirow{2}{*}{\multicolumn{4}{|c|}{$\begin{array}{c}\text { Unknown Sex } \\
\text { Age }\end{array}$}} & \multirow{3}{*}{$\begin{array}{c}\text { Unknown } \\
\text { total }\end{array}$} & \multirow{3}{*}{$\begin{array}{c}\text { Species } \\
\text { total }\end{array}$} \\
\hline & & & & & & & & & & & & & & & & \\
\hline & AHY & SY & ASY & 1 & & $\mathrm{AHY}$ & SY & ASY & 1 & & $\mathrm{AHY}$ & SY & ASY & 1 & & \\
\hline CAHU & 0 & 0 & 0 & 0 & 0 & 0 & 0 & 0 & 1 & 1 & 0 & 0 & 0 & 0 & 0 & 1 \\
\hline RUHU & 1 & 0 & 0 & 1 & 2 & 1 & 0 & 0 & 8 & 9 & 0 & 0 & 0 & 8 & 8 & 19 \\
\hline WEWP & 0 & 0 & 0 & 0 & 0 & 0 & 0 & 0 & 0 & 0 & 2 & 0 & 0 & 0 & 2 & 2 \\
\hline WIFL & 0 & 0 & 0 & 0 & 0 & 0 & 0 & 0 & 0 & 0 & 1 & 0 & 1 & 0 & 2 & 2 \\
\hline HAFL & 0 & 0 & 0 & 0 & 0 & 0 & 0 & 0 & 0 & 0 & 7 & 0 & 0 & 0 & 7 & 7 \\
\hline COFL & 0 & 0 & 0 & 0 & 0 & 0 & 0 & 0 & 0 & 0 & 1 & 0 & 0 & 0 & 1 & 1 \\
\hline PSFL & 0 & 0 & 0 & 0 & 0 & 0 & 0 & 0 & 0 & 0 & 31 & 1 & 1 & 2 & 35 & 35 \\
\hline WAVI & 0 & 0 & 0 & 0 & 0 & 0 & 0 & 0 & 0 & 0 & 41 & 2 & 1 & 2 & 46 & 46 \\
\hline HOWR & 0 & 0 & 0 & 0 & 0 & 1 & 0 & 0 & 0 & 1 & 0 & 0 & 0 & 0 & 0 & 1 \\
\hline RCKI & 2 & 0 & 0 & 0 & 2 & 0 & 0 & 0 & 0 & 0 & 0 & 0 & 0 & 0 & 0 & 2 \\
\hline BGGN & 0 & 0 & 0 & 0 & 0 & 0 & 0 & 0 & 0 & 0 & 0 & 0 & 1 & 0 & 1 & 1 \\
\hline SWTH & 0 & 0 & 0 & 0 & 0 & 0 & 0 & 0 & 0 & 0 & 7 & 1 & 2 & 0 & 10 & 10 \\
\hline HETH & 0 & 0 & 0 & 0 & 0 & 0 & 0 & 0 & 0 & 0 & 5 & 0 & 3 & 0 & 8 & 8 \\
\hline $\mathrm{PHAl}$ & 0 & 1 & 0 & 0 & 1 & 0 & 0 & 0 & 0 & 0 & 0 & 0 & 0 & 0 & 0 & 1 \\
\hline NAWA & 1 & 1 & 0 & 0 & 2 & 4 & 2 & 4 & 0 & 10 & 10 & 0 & 0 & 0 & 10 & 22 \\
\hline YEWA & 0 & 1 & 0 & 0 & 1 & 0 & 1 & 1 & 0 & 2 & 0 & 0 & 0 & 0 & 0 & 3 \\
\hline AUWA & 1 & 0 & 0 & 0 & 1 & 0 & 0 & 0 & 0 & 0 & 1 & 0 & 0 & 0 & 1 & 2 \\
\hline BTYW & 1 & 1 & 0 & 0 & 2 & 1 & 1 & 1 & 0 & 3 & 7 & 0 & 0 & 0 & 7 & 12 \\
\hline TOWA & 1 & 5 & 2 & 0 & 8 & 10 & 0 & 6 & 0 & 16 & 6 & 0 & 0 & 0 & 6 & 30 \\
\hline THWH & 1 & 0 & 1 & 0 & 2 & 0 & 0 & 1 & 0 & 1 & 0 & 0 & 0 & 0 & 0 & 3 \\
\hline HEWA & 0 & 0 & 1 & 0 & 1 & 0 & 1 & 1 & 0 & 2 & 9 & 0 & 0 & 0 & 9 & 12 \\
\hline MGWA & 0 & 0 & 0 & 0 & 0 & 1 & 0 & 0 & 0 & 1 & 1 & 0 & 0 & 0 & 1 & 2 \\
\hline WIWA & 5 & 8 & 6 & 0 & 19 & 20 & 5 & 9 & 0 & 34 & 31 & 0 & 5 & 0 & 36 & 89 \\
\hline $\mathrm{YBCH}$ & 1 & 0 & 0 & 0 & 1 & 0 & 0 & 0 & 0 & 0 & 0 & 0 & 0 & 0 & 0 & 1 \\
\hline WETA & 1 & 0 & 2 & 0 & 3 & 0 & 0 & 1 & 0 & 1 & 0 & 0 & 0 & 0 & 0 & 4 \\
\hline GTTO & 0 & 0 & 0 & 0 & 0 & 0 & 0 & 0 & 0 & 0 & 1 & 0 & 0 & 0 & 1 & 1 \\
\hline $\mathrm{CHSP}$ & 0 & 0 & 0 & 0 & 0 & 0 & 0 & 0 & 0 & 0 & 0 & 3 & 0 & 2 & 5 & 5 \\
\hline BCSP & 0 & 0 & 0 & 0 & 0 & 0 & 0 & 0 & 0 & 0 & 2 & 0 & 0 & 0 & 2 & 2 \\
\hline LISP & 0 & 0 & 0 & 0 & 0 & 0 & 0 & 0 & 0 & 0 & 3 & 0 & 0 & 1 & 4 & 4 \\
\hline WTSP & 0 & 0 & 0 & 0 & 0 & 0 & 0 & 0 & 0 & 0 & 1 & 0 & 0 & 0 & 1 & 1 \\
\hline WCSP & 0 & 0 & 0 & 0 & 0 & 0 & 0 & 0 & 0 & 0 & 29 & 3 & 2 & 2 & 36 & 36 \\
\hline GCSP & 0 & 0 & 0 & 0 & 0 & 0 & 0 & 0 & 0 & 0 & 7 & 2 & 1 & 0 & 10 & 10 \\
\hline BHGR & 4 & 0 & 4 & 0 & 8 & 3 & 2 & 5 & 0 & 10 & 2 & 0 & 0 & 0 & 2 & 20 \\
\hline BUOR & 0 & 0 & 1 & 0 & 1 & 3 & 0 & 1 & 0 & 4 & 1 & 0 & 0 & 0 & 1 & 6 \\
\hline Total & 19 & 17 & 17 & 1 & 54 & 44 & 12 & 30 & 9 & 95 & 206 & 12 & 17 & 17 & 252 & 401 \\
\hline
\end{tabular}


Table B18. Sex and age of individual migrants (banded and unbanded) captured during spring migration, Point Loma banding station, San Diego, California, 2012.

[See appendix A for bird species codes. Age: AHY=after-hatching-year, SY=second-year, ASY=after-second-year, I=Indeterminable]

\begin{tabular}{|c|c|c|c|c|c|c|c|c|c|c|c|c|c|c|}
\hline \multirow{3}{*}{$\begin{array}{l}\text { Species } \\
\text { code }\end{array}$} & \multirow{2}{*}{\multicolumn{3}{|c|}{$\begin{array}{l}\text { Female } \\
\text { Age }\end{array}$}} & \multirow{3}{*}{$\begin{array}{c}\text { Female } \\
\text { total }\end{array}$} & \multirow{2}{*}{\multicolumn{3}{|c|}{$\begin{array}{l}\text { Male } \\
\text { Age }\end{array}$}} & \multirow{3}{*}{$\begin{array}{l}\text { Male } \\
\text { total }\end{array}$} & \multirow{2}{*}{\multicolumn{4}{|c|}{$\begin{array}{c}\text { Unknown sex } \\
\text { Age }\end{array}$}} & \multirow{3}{*}{$\begin{array}{l}\text { Unknown } \\
\text { total }\end{array}$} & \multirow{3}{*}{$\begin{array}{c}\text { Species } \\
\text { total }\end{array}$} \\
\hline & & & & & & & & & & & & & & \\
\hline & AHY & SY & ASY & & AHY & SY & ASY & & AHY & SY & ASY & 1 & & \\
\hline $\mathrm{BCHU}$ & 1 & 0 & 0 & 1 & 1 & 0 & 0 & 1 & 0 & 0 & 0 & 1 & 1 & 3 \\
\hline CAHU & 1 & 0 & 0 & 1 & 1 & 0 & 0 & 1 & 0 & 0 & 0 & 0 & 0 & 2 \\
\hline RUHU & 4 & 0 & 0 & 4 & 8 & 0 & 0 & 8 & 1 & 0 & 0 & 0 & 1 & 13 \\
\hline WEWP & 0 & 0 & 0 & 0 & 0 & 0 & 0 & 0 & 5 & 0 & 0 & 0 & 5 & 5 \\
\hline WIFL & 0 & 0 & 0 & 0 & 0 & 0 & 0 & 0 & 1 & 0 & 0 & 0 & 1 & 1 \\
\hline LEFL & 0 & 0 & 0 & 0 & 0 & 0 & 0 & 0 & 1 & 0 & 0 & 0 & 1 & 1 \\
\hline HAFL & 0 & 0 & 0 & 0 & 0 & 0 & 0 & 0 & 4 & 0 & 0 & 0 & 4 & 4 \\
\hline GRFL & 0 & 0 & 0 & 0 & 0 & 0 & 0 & 0 & 1 & 0 & 0 & 0 & 1 & 1 \\
\hline PSFL & 0 & 0 & 0 & 0 & 0 & 0 & 0 & 0 & 35 & 3 & 0 & 1 & 39 & 39 \\
\hline CAVI & 0 & 0 & 0 & 0 & 0 & 0 & 0 & 0 & 1 & 0 & 0 & 0 & 1 & 1 \\
\hline WAVI & 0 & 0 & 0 & 0 & 0 & 0 & 0 & 0 & 19 & 1 & 0 & 1 & 21 & 21 \\
\hline HOWR & 0 & 0 & 0 & 0 & 0 & 0 & 0 & 0 & 1 & 0 & 0 & 0 & 1 & 1 \\
\hline SWTH & 0 & 0 & 0 & 0 & 0 & 0 & 0 & 0 & 3 & 1 & 0 & 0 & 4 & 4 \\
\hline HETH & 0 & 0 & 0 & 0 & 0 & 0 & 0 & 0 & 3 & 6 & 6 & 0 & 15 & 15 \\
\hline NAWA & 1 & 1 & 0 & 2 & 1 & 0 & 3 & 4 & 1 & 0 & 0 & 0 & 1 & 7 \\
\hline YEWA & 0 & 0 & 0 & 0 & 1 & 0 & 0 & 1 & 0 & 0 & 0 & 0 & 0 & 1 \\
\hline AUWA & 1 & 0 & 1 & 2 & 1 & 1 & 0 & 2 & 0 & 0 & 0 & 0 & 0 & 4 \\
\hline BTYW & 0 & 1 & 0 & 1 & 0 & 1 & 1 & 2 & 0 & 0 & 0 & 0 & 0 & 3 \\
\hline TOWA & 0 & 2 & 2 & 4 & 2 & 2 & 0 & 4 & 1 & 0 & 0 & 0 & 1 & 9 \\
\hline HEWA & 1 & 1 & 1 & 3 & 1 & 3 & 2 & 6 & 0 & 0 & 0 & 0 & 0 & 9 \\
\hline MGWA & 0 & 0 & 0 & 0 & 0 & 0 & 1 & 1 & 1 & 0 & 0 & 0 & 1 & 2 \\
\hline COYE & 1 & 0 & 0 & 1 & 1 & 0 & 0 & 1 & 0 & 0 & 0 & 0 & 0 & 2 \\
\hline WIWA & 9 & 11 & 11 & 31 & 6 & 7 & 17 & 30 & 10 & 1 & 2 & 2 & 15 & 76 \\
\hline WETA & 0 & 0 & 0 & 0 & 0 & 1 & 1 & 2 & 0 & 0 & 0 & 0 & 0 & 2 \\
\hline CHSP & 0 & 0 & 0 & 0 & 0 & 0 & 0 & 0 & 1 & 0 & 0 & 0 & 1 & 1 \\
\hline SAVS & 0 & 0 & 0 & 0 & 0 & 0 & 0 & 0 & 0 & 0 & 0 & 1 & 1 & 1 \\
\hline FOSP & 0 & 0 & 0 & 0 & 0 & 0 & 0 & 0 & 1 & 0 & 0 & 0 & 1 & 1 \\
\hline LISP & 0 & 0 & 0 & 0 & 0 & 0 & 0 & 0 & 6 & 1 & 0 & 0 & 7 & 7 \\
\hline WCSP & 0 & 0 & 0 & 0 & 0 & 0 & 0 & 0 & 28 & 7 & 3 & 0 & 38 & 38 \\
\hline GCSP & 0 & 0 & 0 & 0 & 0 & 0 & 0 & 0 & 3 & 0 & 0 & 0 & 3 & 3 \\
\hline BHGR & 1 & 0 & 0 & 1 & 0 & 0 & 3 & 3 & 0 & 0 & 0 & 0 & 0 & 4 \\
\hline BLGR & 1 & 0 & 0 & 1 & 0 & 0 & 0 & 0 & 0 & 0 & 0 & 0 & 0 & 1 \\
\hline INBU & 0 & 0 & 0 & 0 & 0 & 0 & 0 & 0 & 0 & 1 & 0 & 0 & 1 & 1 \\
\hline BUOR & 3 & 0 & 0 & 3 & 0 & 0 & 0 & 0 & 0 & 0 & 0 & 0 & 0 & 3 \\
\hline Total & 24 & 16 & 15 & 55 & 23 & 15 & 28 & 66 & 127 & 21 & 11 & 6 & 165 & 286 \\
\hline
\end{tabular}


Table B19. Sex and age of individual migrants (banded and unbanded) captured during spring migration, Point Loma banding station, San Diego, California, 2013.

[See appendix A for bird species codes. Age: AHY=after-hatching-year, SY=second-year, ASY=after-second-year]

\begin{tabular}{|c|c|c|c|c|c|c|c|c|c|c|c|c|c|}
\hline \multirow{3}{*}{$\begin{array}{l}\text { Species } \\
\text { code }\end{array}$} & \multirow{2}{*}{\multicolumn{3}{|c|}{$\begin{array}{c}\text { Female } \\
\text { Age }\end{array}$}} & \multirow{3}{*}{$\begin{array}{c}\text { Female } \\
\text { total }\end{array}$} & \multirow{2}{*}{\multicolumn{3}{|c|}{$\begin{array}{l}\text { Male } \\
\text { Age }\end{array}$}} & \multirow{3}{*}{$\begin{array}{l}\text { Male } \\
\text { total }\end{array}$} & \multirow{2}{*}{\multicolumn{3}{|c|}{$\begin{array}{c}\text { Unknown sex } \\
\text { Age }\end{array}$}} & \multirow{3}{*}{$\begin{array}{c}\text { Unknown } \\
\text { total }\end{array}$} & \multirow{3}{*}{$\begin{array}{c}\text { Species } \\
\text { total }\end{array}$} \\
\hline & & & & & & & & & & & & & \\
\hline & AHY & SY & ASY & & AHY & SY & ASY & & AHY & SY & ASY & & \\
\hline SSHA & 0 & 0 & 0 & 0 & 0 & 1 & 0 & 1 & 0 & 0 & 0 & 0 & 1 \\
\hline $\mathrm{BCHU}$ & 3 & 0 & 0 & 3 & 1 & 0 & 0 & 1 & 0 & 0 & 0 & 0 & 4 \\
\hline CAHU & 0 & 0 & 0 & 0 & 2 & 0 & 0 & 2 & 0 & 0 & 0 & 0 & 2 \\
\hline RUHU & 7 & 0 & 0 & 7 & 6 & 2 & 0 & 8 & 0 & 0 & 0 & 0 & 15 \\
\hline WEWP & 0 & 0 & 0 & 0 & 0 & 0 & 0 & 0 & 2 & 0 & 0 & 2 & 2 \\
\hline HAFL & 0 & 0 & 0 & 0 & 0 & 0 & 0 & 0 & 5 & 0 & 0 & 5 & 5 \\
\hline GRFL & 0 & 0 & 0 & 0 & 0 & 0 & 0 & 0 & 1 & 0 & 1 & 2 & 2 \\
\hline PSFL & 0 & 0 & 0 & 0 & 0 & 0 & 0 & 0 & 29 & 6 & 1 & 36 & 36 \\
\hline COFL & 0 & 0 & 0 & 0 & 0 & 0 & 0 & 0 & 1 & 1 & 0 & 2 & 2 \\
\hline WEKI & 0 & 0 & 0 & 0 & 0 & 0 & 1 & 1 & 0 & 0 & 0 & 0 & 1 \\
\hline CAVI & 0 & 0 & 0 & 0 & 0 & 0 & 0 & 0 & 1 & 0 & 0 & 1 & 1 \\
\hline WAVI & 0 & 0 & 0 & 0 & 0 & 0 & 0 & 0 & 11 & 3 & 2 & 16 & 16 \\
\hline HOWR & 0 & 0 & 0 & 0 & 0 & 0 & 0 & 0 & 1 & 0 & 0 & 1 & 1 \\
\hline RCKI & 12 & 4 & 4 & 20 & 3 & 1 & 3 & 7 & 0 & 0 & 0 & 0 & 27 \\
\hline SWTH & 0 & 0 & 0 & 0 & 0 & 0 & 0 & 0 & 1 & 0 & 1 & 2 & 2 \\
\hline HETH & 0 & 0 & 0 & 0 & 0 & 0 & 0 & 0 & 4 & 5 & 1 & 10 & 10 \\
\hline NAWA & 0 & 0 & 1 & 1 & 1 & 1 & 5 & 7 & 0 & 0 & 0 & 0 & 8 \\
\hline AUWA & 0 & 0 & 3 & 3 & 0 & 1 & 1 & 2 & 0 & 0 & 0 & 0 & 5 \\
\hline BTYW & 0 & 1 & 0 & 1 & 0 & 3 & 1 & 4 & 0 & 0 & 0 & 0 & 5 \\
\hline TOWA & 1 & 1 & 0 & 2 & 3 & 0 & 3 & 6 & 0 & 0 & 0 & 0 & 8 \\
\hline HEWA & 1 & 1 & 0 & 2 & 0 & 0 & 1 & 1 & 1 & 0 & 0 & 1 & 4 \\
\hline MGWA & 0 & 0 & 2 & 2 & 1 & 1 & 3 & 5 & 0 & 0 & 0 & 0 & 7 \\
\hline COYE & 0 & 0 & 2 & 2 & 1 & 0 & 0 & 1 & 0 & 0 & 0 & 0 & 3 \\
\hline WIWA & 2 & 1 & 8 & 11 & 8 & 2 & 24 & 34 & 4 & 0 & 2 & 6 & 51 \\
\hline WETA & 0 & 1 & 2 & 3 & 0 & 2 & 0 & 2 & 0 & 0 & 0 & 0 & 5 \\
\hline CHSP & 0 & 0 & 0 & 0 & 0 & 0 & 0 & 0 & 1 & 0 & 0 & 1 & 1 \\
\hline SAVS & 0 & 0 & 0 & 0 & 0 & 0 & 0 & 0 & 0 & 1 & 0 & 1 & 1 \\
\hline FOSP & 0 & 0 & 0 & 0 & 0 & 0 & 0 & 0 & 0 & 0 & 2 & 2 & 2 \\
\hline LISP & 0 & 0 & 0 & 0 & 0 & 0 & 0 & 0 & 10 & 0 & 2 & 12 & 12 \\
\hline WCSP & 0 & 0 & 0 & 0 & 0 & 0 & 0 & 0 & 17 & 15 & 9 & 41 & 41 \\
\hline GCSP & 0 & 0 & 0 & 0 & 0 & 0 & 0 & 0 & 2 & 4 & 0 & 6 & 6 \\
\hline BHGR & 1 & 1 & 2 & 4 & 0 & 5 & 5 & 5 & 0 & 0 & 0 & 0 & 9 \\
\hline BUOR & 0 & 0 & 1 & 1 & 0 & 0 & 0 & 1 & 0 & 0 & 0 & 0 & 2 \\
\hline Total & 27 & 10 & 25 & 62 & 26 & 19 & 47 & 88 & 91 & 35 & 21 & 147 & 297 \\
\hline
\end{tabular}


Table B20. Sex and age of individual migrants (banded and unbanded) captured during spring migration, Point Loma banding station, San Diego, California, 2014.

[See appendix A for bird species codes. Age: $\mathrm{HY}=$ hatching-year, $\mathrm{AHY}=$ =after-hatching-year, $\mathrm{SY}=$ =second-year, $\mathrm{ASY}=$ after-second-year]

\begin{tabular}{|c|c|c|c|c|c|c|c|c|c|c|c|c|c|c|}
\hline \multirow[b]{3}{*}{ Species } & \multirow{2}{*}{\multicolumn{4}{|c|}{$\begin{array}{l}\text { Female } \\
\text { Age }\end{array}$}} & \multirow{3}{*}{$\begin{array}{c}\text { Female } \\
\text { total }\end{array}$} & \multirow{2}{*}{\multicolumn{3}{|c|}{$\begin{array}{l}\text { Male } \\
\text { Age }\end{array}$}} & \multirow{3}{*}{$\begin{array}{l}\text { Male } \\
\text { total }\end{array}$} & \multirow{2}{*}{\multicolumn{3}{|c|}{$\begin{array}{c}\text { Unknown sex } \\
\text { Age }\end{array}$}} & \multirow{3}{*}{$\begin{array}{c}\text { Unknown } \\
\text { total }\end{array}$} & \multirow{3}{*}{$\begin{array}{c}\text { Species } \\
\text { total }\end{array}$} \\
\hline & & & & & & & & & & & & & & \\
\hline & HY & AHY & SY & ASY & & AHY & SY & ASY & & AHY & SY & ASY & & \\
\hline $\mathrm{BCHU}$ & 1 & 0 & 0 & 0 & 1 & 0 & 0 & 0 & 0 & 0 & 0 & 0 & 0 & 1 \\
\hline CAHU & 0 & 6 & 0 & 0 & 6 & 7 & 0 & 0 & 7 & 0 & 0 & 0 & 0 & 13 \\
\hline RUHU & 0 & 14 & 0 & 0 & 14 & 15 & 1 & 0 & 16 & 0 & 0 & 0 & 0 & 30 \\
\hline DOWO & 0 & 1 & 0 & 0 & 1 & 1 & 0 & 0 & 1 & 0 & 0 & 0 & 0 & 2 \\
\hline WEWP & 0 & 0 & 0 & 0 & 0 & 0 & 0 & 0 & 0 & 0 & 1 & 0 & 1 & 1 \\
\hline WIFL & 0 & 0 & 0 & 0 & 0 & 0 & 0 & 0 & 0 & 0 & 1 & 0 & 1 & 1 \\
\hline HAFL & 0 & 0 & 0 & 0 & 0 & 0 & 0 & 0 & 0 & 0 & 8 & 0 & 8 & 8 \\
\hline PSFL & 0 & 1 & 0 & 0 & 1 & 0 & 0 & 0 & 0 & 4 & 93 & 0 & 97 & 98 \\
\hline COFL & 0 & 0 & 0 & 0 & 0 & 0 & 0 & 0 & 0 & 0 & 1 & 0 & 1 & 1 \\
\hline WEKI & 0 & 0 & 0 & 0 & 0 & 0 & 0 & 1 & 1 & 0 & 0 & 0 & 0 & 1 \\
\hline LBVI & 0 & 0 & 0 & 0 & 0 & 0 & 0 & 0 & 0 & 1 & 0 & 0 & 1 & 1 \\
\hline WAVI & 0 & 0 & 0 & 0 & 0 & 0 & 0 & 0 & 0 & 17 & 28 & 3 & 48 & 48 \\
\hline HOWR & 0 & 0 & 0 & 0 & 0 & 0 & 0 & 0 & 0 & 1 & 0 & 0 & 1 & 1 \\
\hline RCKI & 0 & 6 & 5 & 2 & 13 & 3 & 0 & 0 & 3 & 0 & 0 & 0 & 0 & 16 \\
\hline BGGN & 0 & 0 & 0 & 0 & 0 & 0 & 0 & 0 & 0 & 0 & 1 & 0 & 1 & 1 \\
\hline SWTH & 0 & 0 & 0 & 0 & 0 & 0 & 0 & 0 & 0 & 1 & 7 & 0 & 8 & 8 \\
\hline HETH & 0 & 0 & 0 & 0 & 0 & 0 & 0 & 0 & 0 & 2 & 8 & 2 & 12 & 12 \\
\hline NAWA & 0 & 0 & 0 & 1 & 1 & 2 & 0 & 2 & 4 & 0 & 0 & 0 & 0 & 5 \\
\hline YEWA & 0 & 0 & 1 & 0 & 1 & 1 & 1 & 0 & 2 & 2 & 0 & 0 & 2 & 5 \\
\hline AUWA & 0 & 0 & 0 & 1 & 1 & 0 & 1 & 0 & 1 & 0 & 0 & 0 & 0 & 2 \\
\hline BTYW & 0 & 0 & 1 & 2 & 3 & 0 & 2 & 0 & 2 & 0 & 0 & 0 & 0 & 5 \\
\hline TOWA & 0 & 0 & 9 & 0 & 9 & 1 & 10 & 5 & 16 & 0 & 0 & 0 & 0 & 25 \\
\hline THWH & 0 & 0 & 0 & 0 & 0 & 0 & 1 & 1 & 2 & 0 & 0 & 0 & 0 & 2 \\
\hline HEWA & 0 & 0 & 4 & 0 & 4 & 0 & 0 & 1 & 1 & 0 & 0 & 0 & 0 & 5 \\
\hline MGWA & 0 & 1 & 1 & 1 & 3 & 2 & 2 & 2 & 6 & 0 & 0 & 0 & 0 & 9 \\
\hline COYE & 0 & 0 & 0 & 0 & 0 & 1 & 0 & 3 & 4 & 0 & 0 & 0 & 0 & 4 \\
\hline WIWA & 0 & 7 & 12 & 12 & 31 & 3 & 6 & 19 & 28 & 0 & 6 & 1 & 7 & 66 \\
\hline WETA & 0 & 0 & 0 & 0 & 0 & 0 & 3 & 0 & 3 & 0 & 1 & 0 & 1 & 4 \\
\hline CHSP & 0 & 0 & 0 & 0 & 0 & 0 & 0 & 0 & 0 & 0 & 2 & 0 & 2 & 2 \\
\hline SAGS & 0 & 0 & 0 & 0 & 0 & 0 & 0 & 0 & 0 & 0 & 1 & 0 & 1 & 1 \\
\hline FOSP & 0 & 0 & 0 & 0 & 0 & 0 & 0 & 0 & 0 & 0 & 5 & 0 & 5 & 5 \\
\hline LISP & 0 & 0 & 0 & 0 & 0 & 0 & 0 & 0 & 0 & 0 & 1 & 0 & 1 & 1 \\
\hline WCSP & 0 & 0 & 0 & 0 & 0 & 0 & 0 & 0 & 0 & 6 & 10 & 4 & 20 & 20 \\
\hline GCSP & 0 & 0 & 0 & 0 & 0 & 0 & 0 & 0 & 0 & 1 & 3 & 0 & 4 & 4 \\
\hline ORJU & 0 & 0 & 0 & 0 & 0 & 0 & 0 & 0 & 0 & 0 & 1 & 0 & 1 & 1 \\
\hline BHGR & 0 & 0 & 0 & 1 & 1 & 0 & 1 & 1 & 2 & 0 & 0 & 0 & 0 & 3 \\
\hline Total & 1 & 36 & 33 & 20 & 90 & 36 & 28 & 35 & 99 & 35 & 178 & 10 & 223 & 412 \\
\hline
\end{tabular}


Table B21. Sex and age of individual migrants (banded and unbanded) captured during spring migration, Point Loma banding station, San Diego, California, 2015.

[See appendix A for bird species codes. Age: $\mathrm{HY}=$ hatching-year, $\mathrm{AHY}=$ =after-hatching-year, $\mathrm{SY}=$ second-year, $\mathrm{ASY}=$ after-second-year, I=indeterminable]

\begin{tabular}{|c|c|c|c|c|c|c|c|c|c|c|c|c|c|c|c|c|c|}
\hline \multirow[b]{3}{*}{ Species } & \multirow{2}{*}{\multicolumn{4}{|c|}{$\begin{array}{c}\text { Female } \\
\text { Age } \\
\end{array}$}} & \multirow{3}{*}{$\begin{array}{c}\text { Female } \\
\text { total }\end{array}$} & \multirow{2}{*}{\multicolumn{4}{|c|}{$\begin{array}{l}\text { Male } \\
\text { Age } \\
\end{array}$}} & \multirow{3}{*}{$\begin{array}{l}\text { Male } \\
\text { total }\end{array}$} & \multirow{2}{*}{\multicolumn{5}{|c|}{$\begin{array}{c}\text { Unknown Sex } \\
\text { Age }\end{array}$}} & \multirow{3}{*}{$\begin{array}{c}\text { Unknown } \\
\text { total }\end{array}$} & \multirow{3}{*}{$\begin{array}{c}\text { Species } \\
\text { total }\end{array}$} \\
\hline & & & & & & & & & & & & & & & & & \\
\hline & $\mathrm{AHY}$ & sY & ASY & 1 & & $\mathrm{AHY}$ & SY & ASY & 1 & & HY & AHY & sY & ASY & 1 & & \\
\hline AMKE & 0 & 0 & 0 & 0 & 0 & 1 & 0 & 0 & 0 & 1 & 0 & 0 & 0 & 0 & 0 & 0 & 1 \\
\hline $\mathrm{BCHU}$ & 3 & 0 & 0 & 1 & 4 & 1 & 0 & 0 & 0 & 1 & 1 & 0 & 0 & 0 & 0 & 1 & 6 \\
\hline CAHU & 5 & 0 & 0 & 0 & 5 & 6 & 0 & 0 & 1 & 7 & 0 & 0 & 0 & 0 & 0 & 0 & 12 \\
\hline RUHU & 10 & 0 & 0 & 1 & 11 & 10 & 0 & 0 & 1 & 11 & 0 & 0 & 0 & 0 & 0 & 0 & 22 \\
\hline WEWP & 0 & 0 & 0 & 0 & 0 & 0 & 0 & 0 & 0 & 0 & 0 & 6 & 0 & 0 & 0 & 6 & 6 \\
\hline WIFL & 0 & 0 & 0 & 0 & 0 & 0 & 0 & 0 & 0 & 0 & 0 & 3 & 0 & 0 & 0 & 3 & 3 \\
\hline HAFL & 0 & 0 & 0 & 0 & 0 & 0 & 0 & 0 & 0 & 0 & 0 & 15 & 0 & 0 & 0 & 15 & 15 \\
\hline DUFL & 0 & 0 & 0 & 0 & 0 & 0 & 0 & 0 & 0 & 0 & 0 & 1 & 0 & 0 & 0 & 1 & 1 \\
\hline PSFL & 0 & 0 & 1 & 0 & 1 & 0 & 0 & 0 & 0 & 0 & 0 & 69 & 3 & 1 & 1 & 74 & 75 \\
\hline COFL & 0 & 0 & 0 & 0 & 0 & 0 & 0 & 0 & 0 & 0 & 0 & 5 & 0 & 0 & 0 & 5 & 5 \\
\hline SAPH & 1 & 0 & 0 & 0 & 1 & 0 & 0 & 0 & 0 & 0 & 1 & 0 & 0 & 0 & 0 & 1 & 2 \\
\hline CAVI & 0 & 0 & 0 & 0 & 0 & 0 & 0 & 0 & 0 & 0 & 0 & 0 & 1 & 0 & 0 & 1 & 1 \\
\hline WAVI & 0 & 0 & 0 & 0 & 0 & 0 & 0 & 0 & 0 & 0 & 0 & 33 & 5 & 8 & 0 & 46 & 46 \\
\hline RCKI & 5 & 0 & 0 & 0 & 5 & 0 & 0 & 0 & 0 & 0 & 0 & 0 & 0 & 0 & 0 & 0 & 5 \\
\hline SWTH & 0 & 0 & 0 & 0 & 0 & 0 & 0 & 0 & 0 & 0 & 0 & 0 & 0 & 2 & 0 & 2 & 2 \\
\hline HETH & 0 & 0 & 0 & 0 & 0 & 0 & 0 & 0 & 0 & 0 & 0 & 6 & 3 & 1 & 0 & 10 & 10 \\
\hline GRCA & 0 & 0 & 0 & 0 & 0 & 0 & 0 & 0 & 0 & 0 & 0 & 0 & 0 & 1 & 0 & 1 & 1 \\
\hline NAWA & 0 & 0 & 2 & 0 & 2 & 1 & 2 & 1 & 0 & 4 & 0 & 1 & 1 & 0 & 0 & 2 & 8 \\
\hline YEWA & 2 & 1 & 0 & 0 & 3 & 0 & 1 & 0 & 0 & 1 & 0 & 1 & 0 & 0 & 0 & 1 & 5 \\
\hline BTYW & 1 & 4 & 0 & 0 & 5 & 1 & 1 & 1 & 0 & 3 & 0 & 0 & 0 & 0 & 0 & 0 & 8 \\
\hline TOWA & 1 & 3 & 1 & 0 & 5 & 0 & 3 & 6 & 0 & 9 & 0 & 0 & 0 & 0 & 0 & 0 & 14 \\
\hline HEWA & 0 & 0 & 0 & 0 & 0 & 0 & 1 & 1 & 0 & 2 & 0 & 0 & 0 & 0 & 0 & 0 & 2 \\
\hline MGWA & 0 & 0 & 1 & 0 & 1 & 0 & 0 & 0 & 0 & 0 & 0 & 0 & 0 & 0 & 0 & 0 & 1 \\
\hline COYE & 1 & 0 & 0 & 0 & 1 & 0 & 0 & 0 & 0 & 0 & 0 & 0 & 0 & 0 & 0 & 0 & 1 \\
\hline WIWA & 6 & 10 & 15 & 0 & 31 & 11 & 9 & 30 & 0 & 50 & 0 & 1 & 0 & 0 & 0 & 1 & 82 \\
\hline CAWA & 1 & 0 & 0 & 0 & 1 & 0 & 0 & 0 & 0 & 0 & 0 & 0 & 0 & 0 & 0 & 0 & 1 \\
\hline WETA & 0 & 1 & 2 & 0 & 3 & 0 & 0 & 0 & 0 & 0 & 0 & 0 & 0 & 0 & 0 & 0 & 3 \\
\hline CHSP & 0 & 0 & 0 & 0 & 0 & 0 & 0 & 0 & 0 & 0 & 0 & 0 & 0 & 1 & 0 & 1 & 1 \\
\hline LISP & 0 & 0 & 0 & 0 & 0 & 0 & 0 & 0 & 0 & 0 & 0 & 4 & 0 & 0 & 0 & 4 & 4 \\
\hline WCSP & 0 & 0 & 0 & 0 & 0 & 0 & 0 & 0 & 0 & 0 & 0 & 8 & 3 & 0 & 0 & 11 & 11 \\
\hline GCSP & 0 & 0 & 0 & 0 & 0 & 0 & 0 & 0 & 0 & 0 & 0 & 4 & 0 & 0 & 0 & 4 & 4 \\
\hline BHGR & 0 & 0 & 3 & 0 & 3 & 0 & 1 & 1 & 0 & 2 & 0 & 0 & 0 & 0 & 0 & 0 & 5 \\
\hline BUOR & 0 & 0 & 1 & 0 & 1 & 0 & 0 & 0 & 0 & 0 & 0 & 0 & 0 & 0 & 0 & 0 & 1 \\
\hline Total & 36 & 19 & 26 & 2 & 83 & 31 & 18 & 40 & 2 & 91 & 2 & 157 & 16 & 14 & 1 & 190 & 364 \\
\hline
\end{tabular}


Table B22. First and median arrival dates per migrant species during spring migration, Point Loma banding station, San Diego, California, 2011-15.

[See appendix A for bird species codes. Unidentified species were not included in analysis. Median was calculated for species with at least two individuals captured]

\begin{tabular}{|c|c|c|c|c|c|c|c|c|c|c|c|c|c|c|c|c|c|}
\hline \multirow[b]{2}{*}{ Species } & \multicolumn{6}{|c|}{ First arrival date } & \multicolumn{6}{|c|}{ Median arrival date } & \multicolumn{5}{|c|}{ Number of captures } \\
\hline & 2011 & 2012 & 2013 & 2014 & 2015 & Average & 2011 & 2012 & 2013 & 2014 & 2015 & Average & 2011 & 2012 & 2013 & 2014 & 2015 \\
\hline SSHA & & & $4 / 16$ & & & $4 / 16$ & & & $4 / 16$ & & & $5 / 5$ & & & 1 & & \\
\hline AMKE & & & & & $5 / 5$ & $5 / 5$ & & & & & $5 / 5$ & $4 / 16$ & & & & & 1 \\
\hline $\mathrm{BCHU}$ & & $4 / 9$ & $4 / 3$ & $5 / 15$ & $4 / 10$ & $4 / 17$ & & $4 / 9$ & $4 / 17$ & $5 / 15$ & $4 / 27$ & $5 / 5$ & & 3 & 4 & 1 & 6 \\
\hline $\mathrm{CAHU}$ & $4 / 28$ & $4 / 17$ & $4 / 18$ & $4 / 8$ & $4 / 3$ & $4 / 15$ & $4 / 28$ & $4 / 27$ & $4 / 18$ & $4 / 15$ & $4 / 27$ & $4 / 25$ & 1 & 2 & 2 & 13 & 12 \\
\hline RUHU & $4 / 4$ & $4 / 4$ & $4 / 1$ & $4 / 7$ & $4 / 3$ & $4 / 4$ & $4 / 15$ & $4 / 17$ & $4 / 18$ & $4 / 9$ & $4 / 9$ & $4 / 23$ & 19 & 13 & 15 & 30 & 22 \\
\hline DOWO & & & & $4 / 3$ & & $4 / 3$ & & & & $4 / 24$ & & $4 / 14$ & & & & 2 & \\
\hline WEWP & $5 / 12$ & $5 / 15$ & $5 / 14$ & $5 / 27$ & $4 / 21$ & $5 / 12$ & $5 / 19$ & $5 / 29$ & $5 / 14$ & $5 / 27$ & $5 / 26$ & $4 / 24$ & 2 & 5 & 2 & 1 & 6 \\
\hline WIFL & $5 / 26$ & $5 / 29$ & & $5 / 27$ & $5 / 26$ & $5 / 27$ & $5 / 26$ & $5 / 29$ & & $5 / 27$ & $5 / 26$ & $5 / 23$ & 2 & 1 & & 1 & 3 \\
\hline LEFL & & $4 / 20$ & & & & $4 / 20$ & & $4 / 20$ & & & & $5 / 27$ & & 1 & & & \\
\hline HAFL & $4 / 20$ & $4 / 3$ & $4 / 3$ & $4 / 9$ & $4 / 15$ & $4 / 10$ & $4 / 22$ & $4 / 11$ & $4 / 12$ & $4 / 19$ & $4 / 20$ & $4 / 21$ & 7 & 4 & 5 & 8 & 15 \\
\hline GRFL & & $4 / 16$ & $4 / 24$ & & & $4 / 20$ & & $4 / 16$ & $5 / 4$ & & & $4 / 17$ & & 1 & 2 & & \\
\hline DUFL & & & & & $4 / 21$ & $4 / 21$ & & & & & $4 / 21$ & $4 / 26$ & & & & & 1 \\
\hline COFL & $5 / 26$ & & $4 / 12$ & $4 / 8$ & $4 / 10$ & $4 / 22$ & $5 / 26$ & & $4 / 28$ & $4 / 8$ & $4 / 20$ & $4 / 21$ & 1 & & 2 & 1 & 5 \\
\hline PSFL & $4 / 20$ & $4 / 4$ & $4 / 3$ & $4 / 8$ & $4 / 10$ & $4 / 9$ & $4 / 28$ & $5 / 15$ & $4 / 23$ & $5 / 27$ & $4 / 22$ & $5 / 5$ & 35 & 39 & 36 & 98 & 76 \\
\hline SAPH & & & & & $4 / 28$ & $4 / 28$ & & & & & $4 / 28$ & $4 / 28$ & & & & & 2 \\
\hline WEKI & & & $4 / 11$ & $4 / 10$ & & $4 / 11$ & & & $4 / 11$ & $4 / 10$ & & $4 / 11$ & & & 1 & 1 & \\
\hline LBVI & & & & $5 / 27$ & & $5 / 27$ & & & & $5 / 27$ & & $5 / 27$ & & & & 1 & \\
\hline CAVI & & $4 / 10$ & $5 / 14$ & & $4 / 23$ & $4 / 26$ & & $4 / 10$ & $5 / 14$ & & $4 / 23$ & $4 / 26$ & & 1 & 1 & & 1 \\
\hline WAVI & $4 / 6$ & $4 / 6$ & $4 / 11$ & $4 / 2$ & $4 / 14$ & $4 / 8$ & $4 / 28$ & $5 / 8$ & $4 / 26$ & $5 / 9$ & $5 / 5$ & $5 / 3$ & 47 & 21 & 16 & 48 & 47 \\
\hline HOWR & $4 / 11$ & $4 / 9$ & $4 / 1$ & $4 / 10$ & & $4 / 8$ & $4 / 11$ & $4 / 9$ & $4 / 1$ & $4 / 10$ & & $4 / 8$ & 1 & 1 & 2 & 1 & \\
\hline RCKI & $4 / 8$ & & $4 / 1$ & $4 / 1$ & $4 / 15$ & $4 / 6$ & $4 / 9$ & & $4 / 12$ & $4 / 9$ & $4 / 21$ & $4 / 13$ & 2 & & 30 & 19 & 6 \\
\hline BGGN & $4 / 6$ & & & $4 / 2$ & & $4 / 4$ & $4 / 6$ & & & $4 / 2$ & & $4 / 4$ & 1 & & & 1 & \\
\hline SWTH & $4 / 22$ & $5 / 8$ & $5 / 14$ & $5 / 9$ & $4 / 20$ & $5 / 3$ & $5 / 12$ & $5 / 15$ & $5 / 14$ & $5 / 15$ & $4 / 24$ & $5 / 10$ & 10 & 4 & 2 & 8 & 2 \\
\hline HETH & $4 / 4$ & $4 / 2$ & $4 / 1$ & $4 / 2$ & $4 / 2$ & $4 / 2$ & $4 / 10$ & $4 / 3$ & $4 / 5$ & $4 / 10$ & $4 / 13$ & $4 / 9$ & 8 & 21 & 10 & 15 & 11 \\
\hline GRCA & & & & & $5 / 5$ & $5 / 5$ & & & & & $5 / 5$ & $5 / 5$ & & & & & 1 \\
\hline PHAI & $5 / 5$ & & & & & $5 / 5$ & $5 / 5$ & & & & & $5 / 5$ & 1 & & & & \\
\hline NAWA & $4 / 6$ & $4 / 17$ & $4 / 3$ & $4 / 8$ & $4 / 13$ & $4 / 9$ & $4 / 24$ & $4 / 20$ & $4 / 11$ & $4 / 18$ & $4 / 16$ & $4 / 18$ & 24 & 7 & 8 & 5 & 8 \\
\hline YEWA & $5 / 5$ & $4 / 24$ & & $5 / 15$ & $5 / 12$ & $5 / 7$ & $5 / 26$ & $4 / 24$ & & $5 / 15$ & $5 / 26$ & $5 / 16$ & 3 & 1 & & 5 & 5 \\
\hline AUWA & $4 / 11$ & $4 / 5$ & $4 / 5$ & $4 / 8$ & & $4 / 7$ & $4 / 19$ & $4 / 15$ & $4 / 10$ & $4 / 12$ & & $4 / 14$ & 2 & 4 & 5 & 2 & \\
\hline BTYW & $4 / 15$ & $4 / 9$ & $4 / 8$ & $4 / 7$ & $4 / 9$ & $4 / 10$ & $4 / 26$ & $4 / 16$ & $4 / 10$ & $4 / 10$ & $4 / 19$ & $4 / 16$ & 12 & 3 & 5 & 5 & 8 \\
\hline TOWA & $4 / 7$ & $4 / 23$ & $4 / 18$ & $4 / 7$ & $4 / 10$ & $4 / 13$ & $4 / 27$ & $5 / 8$ & $4 / 23$ & $4 / 22$ & $4 / 27$ & $4 / 28$ & 30 & 9 & 8 & 26 & 14 \\
\hline THWH & $4 / 26$ & & & $4 / 21$ & & $4 / 24$ & $5 / 8$ & & & $4 / 21$ & & $4 / 30$ & 3 & & & 2 & \\
\hline HEWA & $4 / 21$ & $4 / 18$ & $4 / 19$ & $4 / 14$ & $4 / 23$ & $4 / 19$ & $4 / 27$ & $4 / 23$ & $5 / 4$ & $5 / 9$ & $5 / 2$ & $5 / 1$ & 12 & 9 & 4 & 5 & 2 \\
\hline MGWA & $4 / 27$ & $4 / 18$ & $4 / 18$ & $4 / 14$ & $4 / 17$ & $4 / 19$ & $5 / 11$ & $4 / 20$ & $4 / 19$ & $5 / 15$ & $4 / 17$ & $4 / 29$ & 2 & 2 & 7 & 9 & 1 \\
\hline COYE & & $4 / 16$ & $4 / 4$ & $4 / 4$ & $4 / 21$ & $4 / 11$ & & $4 / 16$ & $4 / 17$ & $4 / 9$ & $4 / 21$ & $4 / 17$ & & 2 & 3 & 4 & 1 \\
\hline WIWA & $4 / 5$ & $4 / 3$ & $4 / 3$ & $4 / 3$ & $4 / 10$ & $4 / 5$ & $4 / 29$ & $5 / 8$ & $4 / 22$ & $4 / 24$ & $4 / 27$ & $4 / 28$ & 90 & 77 & 53 & 68 & 82 \\
\hline CAWA & & & & & $5 / 26$ & $5 / 26$ & & & & & $5 / 26$ & $5 / 26$ & & & & & 1 \\
\hline $\mathrm{YBCH}$ & $5 / 5$ & & & & & $5 / 5$ & $5 / 5$ & & & & & $5 / 5$ & 1 & & & & \\
\hline WETA & $4 / 18$ & $4 / 20$ & $4 / 24$ & $4 / 28$ & $5 / 12$ & $4 / 26$ & $5 / 12$ & $5 / 2$ & $5 / 14$ & $5 / 12$ & $5 / 12$ & $5 / 11$ & 4 & 2 & 5 & 4 & 3 \\
\hline GTTO & $4 / 28$ & & & & & $4 / 28$ & $4 / 28$ & & & & & $4 / 28$ & 1 & & & & \\
\hline
\end{tabular}




\begin{tabular}{|c|c|c|c|c|c|c|c|c|c|c|c|c|c|c|c|c|c|}
\hline \multirow[b]{2}{*}{ Species } & \multicolumn{6}{|c|}{ First arrival date } & \multicolumn{6}{|c|}{ Median arrival date } & \multicolumn{5}{|c|}{ Number of captures } \\
\hline & 2011 & 2012 & 2013 & 2014 & 2015 & Average & 2011 & 2012 & 2013 & 2014 & 2015 & Average & 2011 & 2012 & 2013 & 2014 & 2015 \\
\hline CHSP & $4 / 15$ & $4 / 23$ & $4 / 24$ & $4 / 7$ & $4 / 2$ & $4 / 14$ & $4 / 29$ & $4 / 23$ & $4 / 24$ & $4 / 7$ & $4 / 2$ & $4 / 17$ & 5 & 1 & 1 & 2 & 1 \\
\hline BCSP & $4 / 26$ & & & & & $4 / 26$ & $4 / 26$ & & & & & $4 / 26$ & 2 & & & & \\
\hline SAGS & & & & $5 / 15$ & & $5 / 15$ & & & & $5 / 15$ & & $5 / 15$ & & & & 1 & \\
\hline SAVS & & $4 / 16$ & $4 / 19$ & & & $4 / 18$ & & $4 / 16$ & $4 / 19$ & & & $4 / 18$ & & 1 & 1 & & \\
\hline FOSP & & $4 / 20$ & $4 / 2$ & $4 / 3$ & & $4 / 8$ & & $4 / 20$ & $4 / 3$ & $4 / 10$ & & $4 / 11$ & & 1 & 2 & 5 & \\
\hline LISP & $4 / 6$ & $4 / 3$ & $4 / 1$ & $4 / 15$ & $4 / 1$ & $4 / 5$ & $4 / 11$ & $4 / 13$ & $4 / 11$ & $4 / 15$ & $4 / 5$ & $4 / 11$ & 4 & 8 & 12 & 1 & 4 \\
\hline WTSP & $5 / 5$ & & & & & $5 / 5$ & $5 / 5$ & & & & & $5 / 5$ & 1 & & & & \\
\hline WCSP & $4 / 4$ & $4 / 2$ & $4 / 9$ & $4 / 1$ & $4 / 1$ & $4 / 3$ & $4 / 20$ & $4 / 16$ & $4 / 18$ & $4 / 10$ & $4 / 13$ & $4 / 16$ & 37 & 38 & 41 & 20 & 12 \\
\hline GCSP & $4 / 4$ & $4 / 5$ & $4 / 1$ & $4 / 1$ & $4 / 8$ & $4 / 4$ & $4 / 16$ & $4 / 6$ & $4 / 8$ & $4 / 12$ & $4 / 11$ & $4 / 12$ & 11 & 3 & 7 & 5 & 4 \\
\hline ORJU & & & & $4 / 14$ & & $4 / 14$ & & & & $4 / 14$ & & $4 / 14$ & & & & 1 & \\
\hline $\mathrm{BHGR}$ & $4 / 4$ & $4 / 3$ & $4 / 5$ & $4 / 4$ & $4 / 20$ & $4 / 7$ & $4 / 25$ & $4 / 17$ & $4 / 19$ & $4 / 24$ & $4 / 27$ & $4 / 23$ & 20 & 4 & 9 & 3 & 5 \\
\hline BLGR & & $4 / 19$ & & & & $4 / 19$ & & $4 / 19$ & & & & $4 / 20$ & & 1 & & & \\
\hline INBU & & $5 / 29$ & & & & $5 / 29$ & & $5 / 29$ & & & & $5 / 30$ & & 1 & & & \\
\hline BUOR & $4 / 15$ & $4 / 16$ & $4 / 19$ & & $4 / 15$ & $4 / 16$ & $4 / 24$ & $4 / 17$ & $4 / 19$ & & $4 / 15$ & $4 / 19$ & 6 & 3 & 2 & & 1 \\
\hline
\end{tabular}




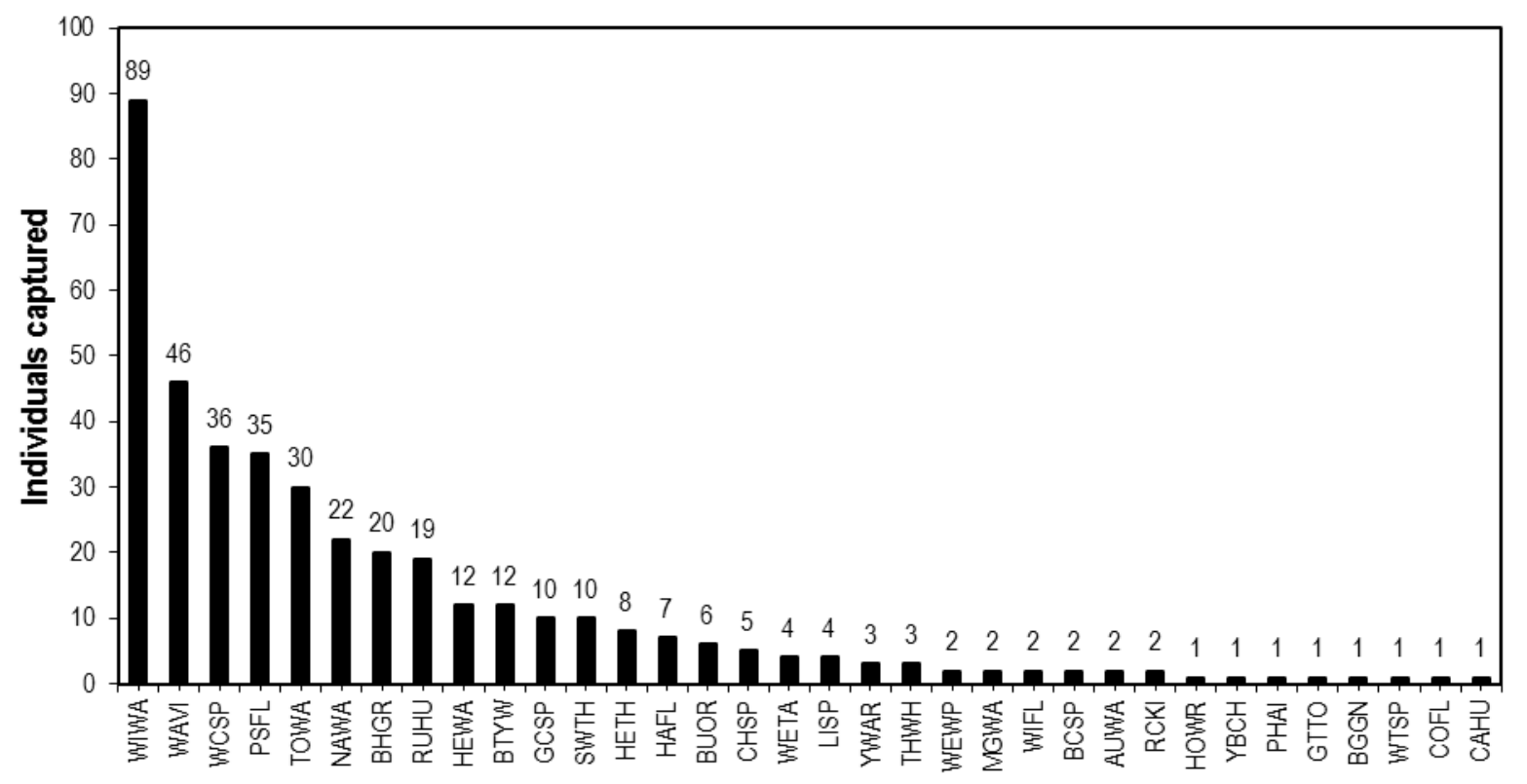

Species

Figure B1. Number of individual migrants captured per species during spring migration, Point Loma banding station, San Diego, California, 2011. See Appendix A for four-letter bird species codes.

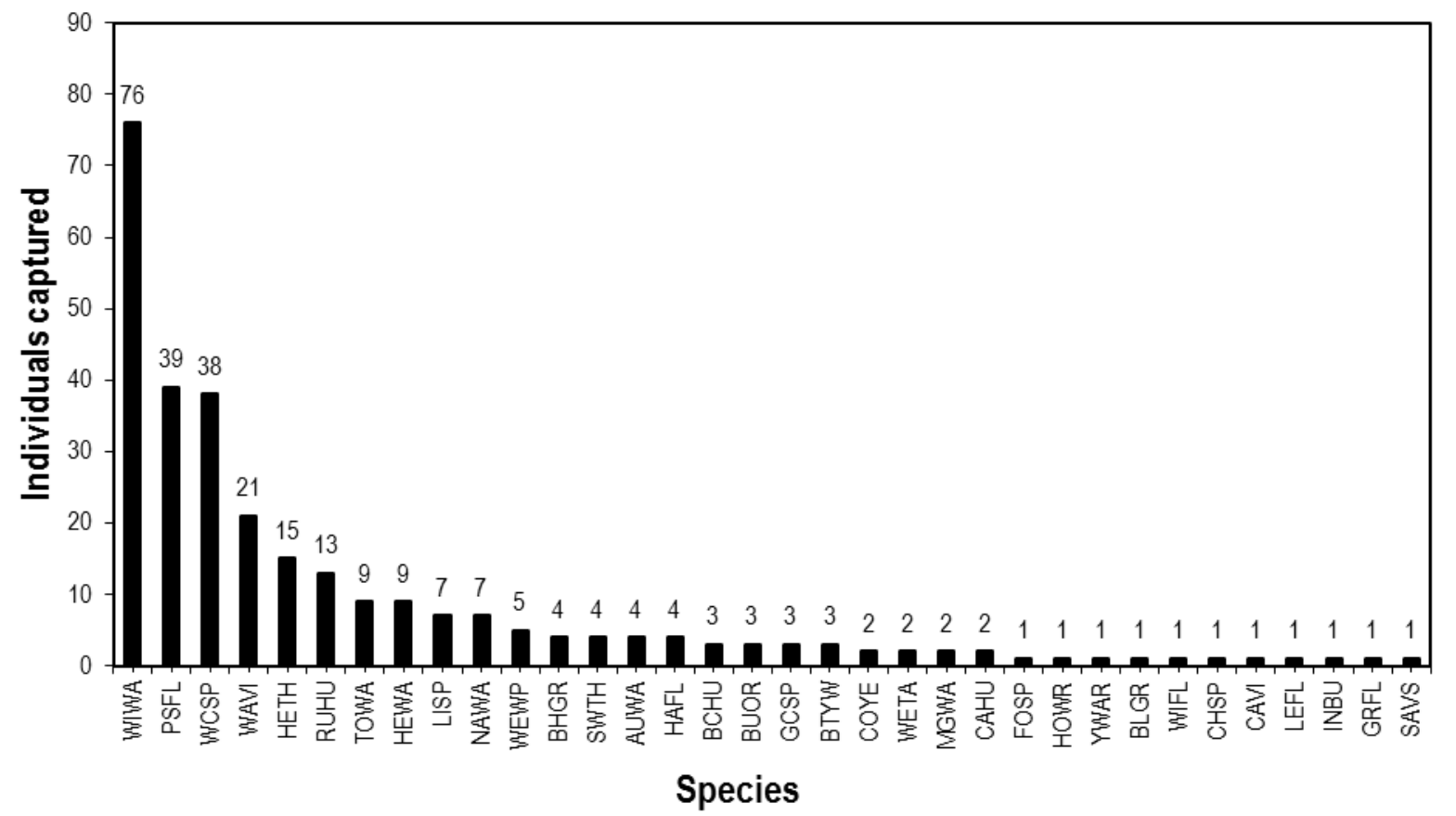

Figure B2. Number of individual migrants captured per species during spring migration, Point Loma banding station, San Diego, California, 2012. See Appendix A for four-letter bird species codes. 


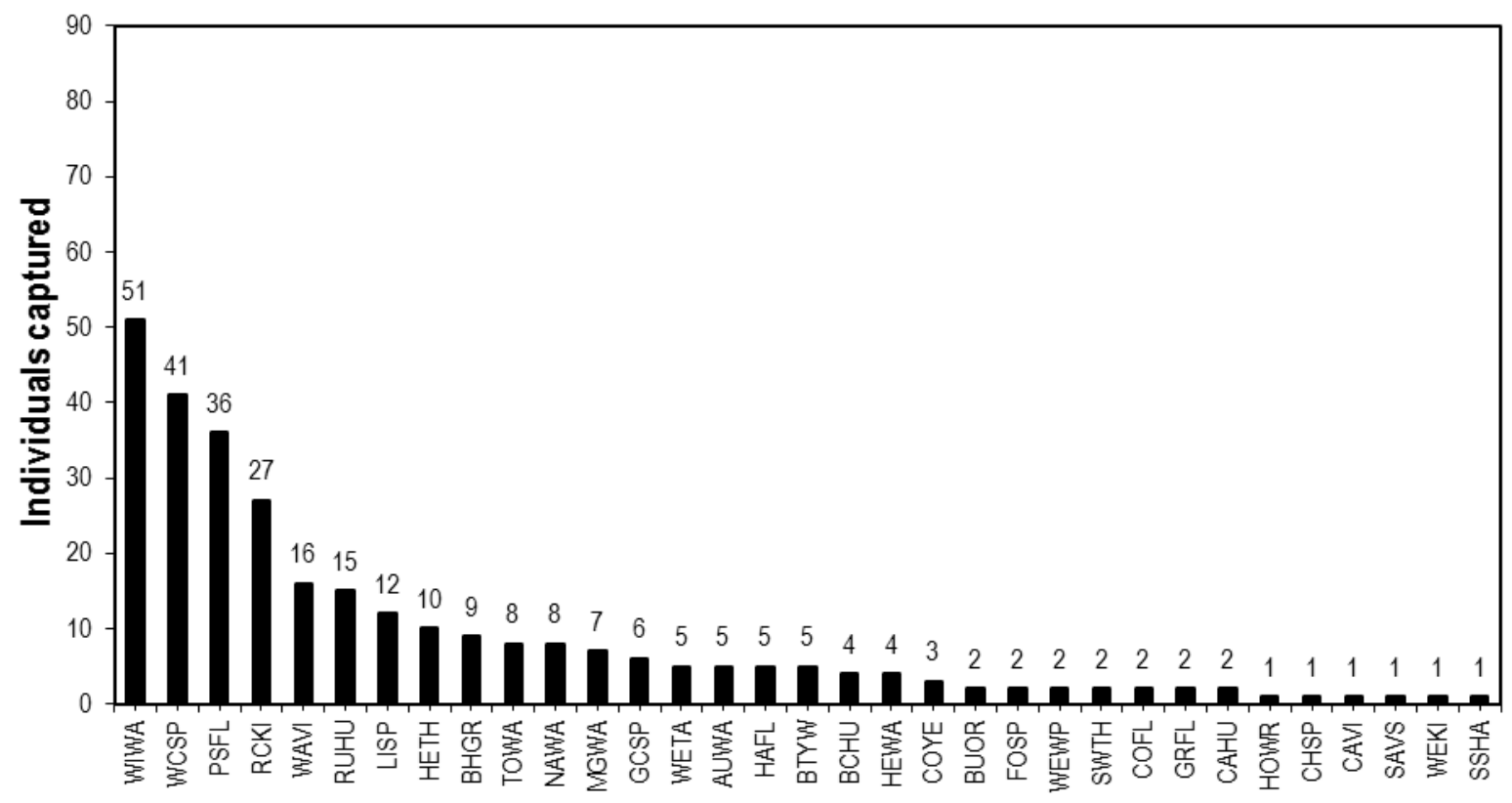

Species

Figure B3. Number of individual migrants captured per species during spring migration, Point Loma banding station, San Diego, California, 2013. See Appendix A for four-letter bird species codes.

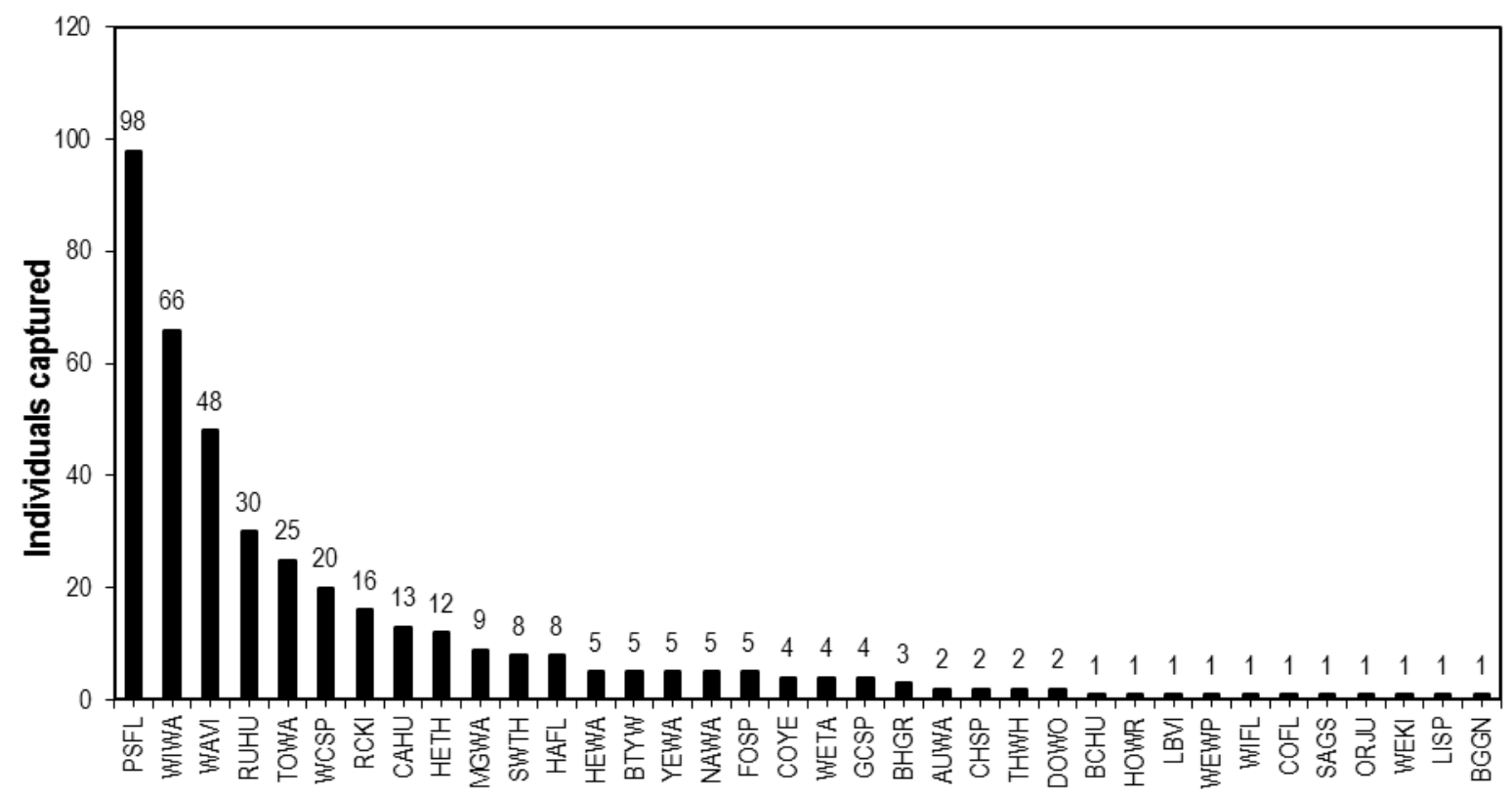

Species

Figure B4. Number of individual migrants captured per species during spring migration, Point Loma banding station, San Diego, California, 2014. See Appendix A for four-letter bird species codes. 


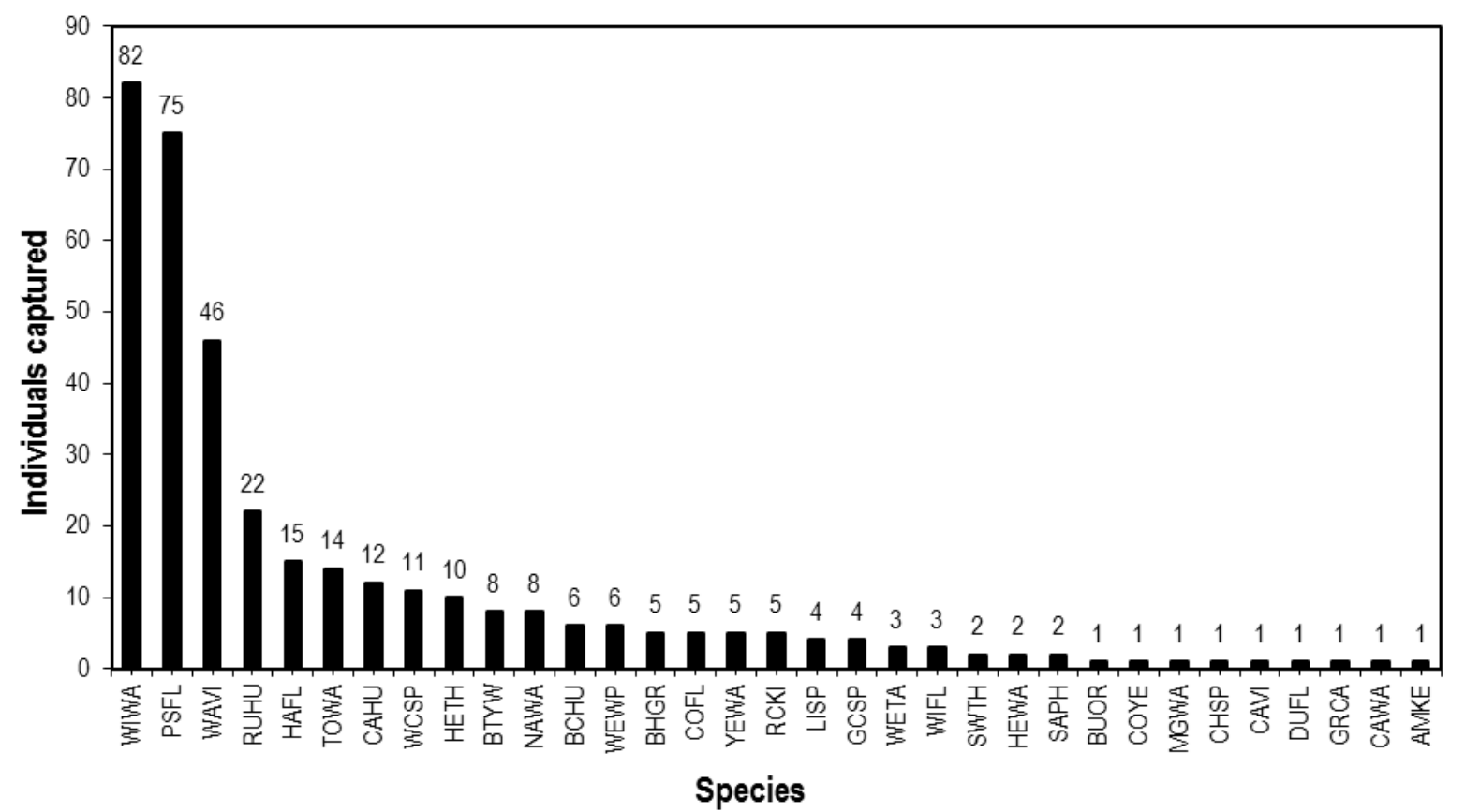

Figure B5. Number of individual migrants captured per species during spring migration, Point Loma banding station, San Diego, California, 2015. See Appendix A for four-letter bird species codes.

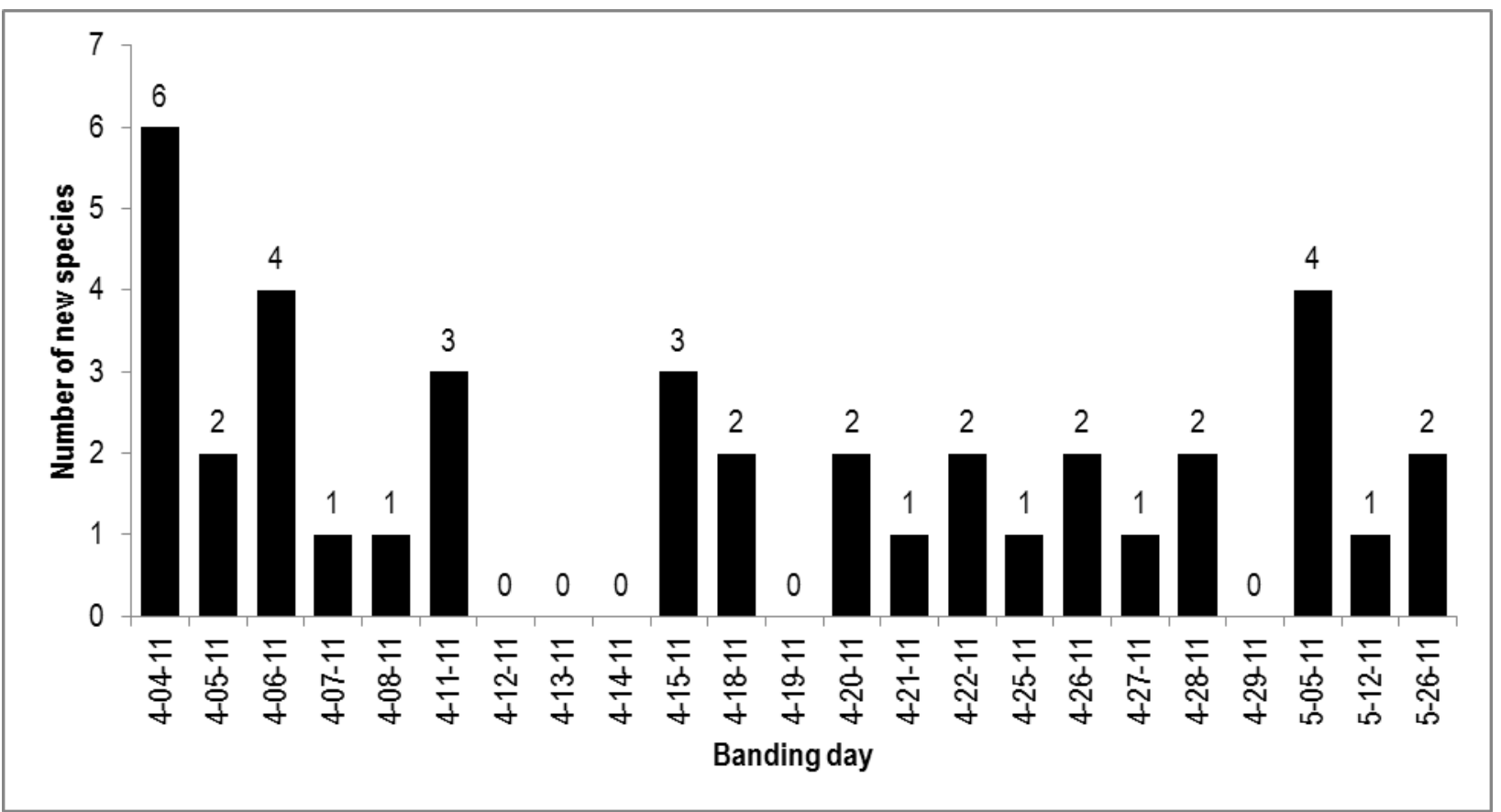

Figure B6. Number of new migrant species arriving per banding day during spring migration, Point Loma banding station, San Diego, California, 2011. 


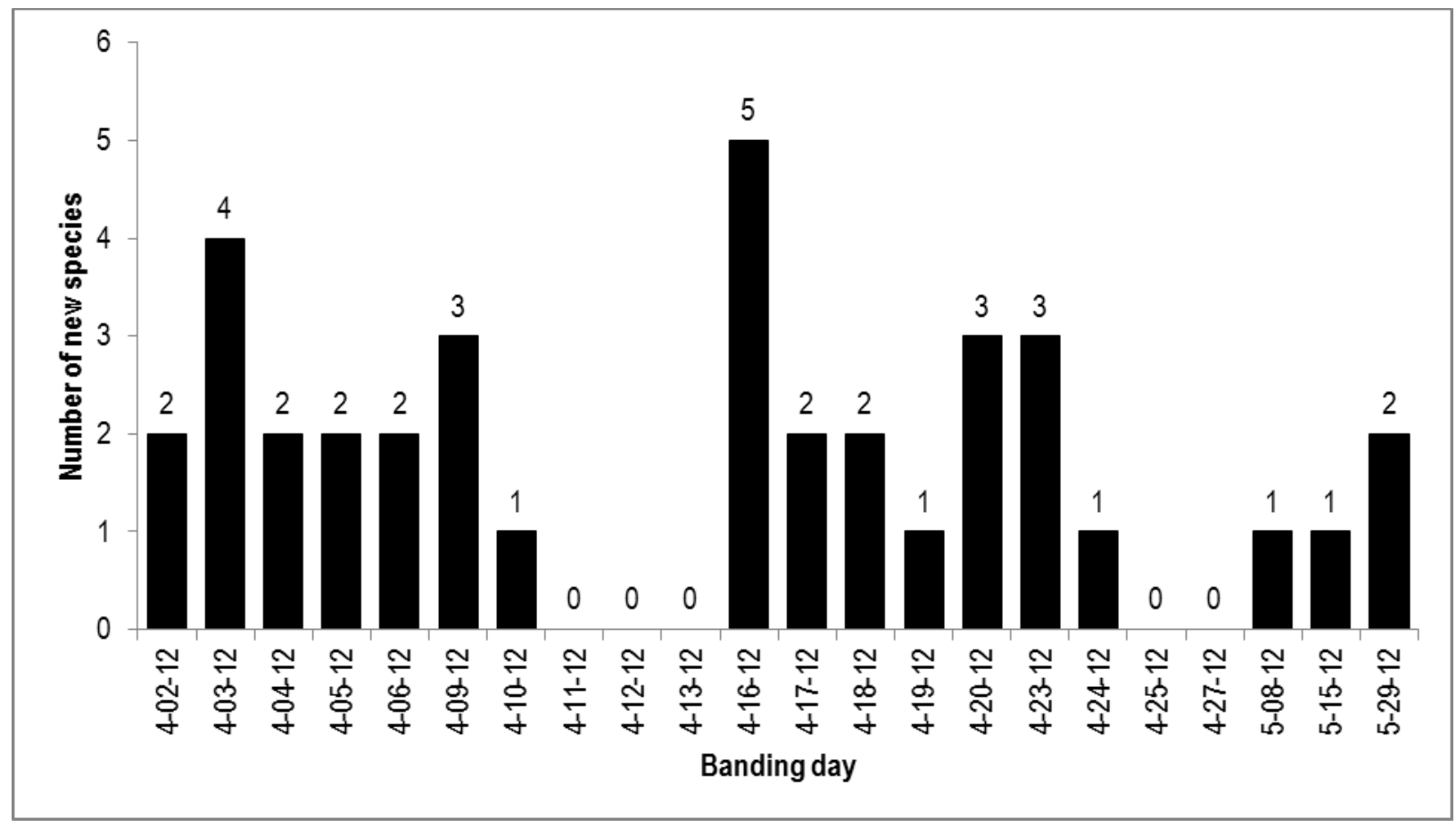

Figure B7. Number of new migrant species arriving per banding day during spring migration, Point Loma banding station, San Diego, California, 2012.

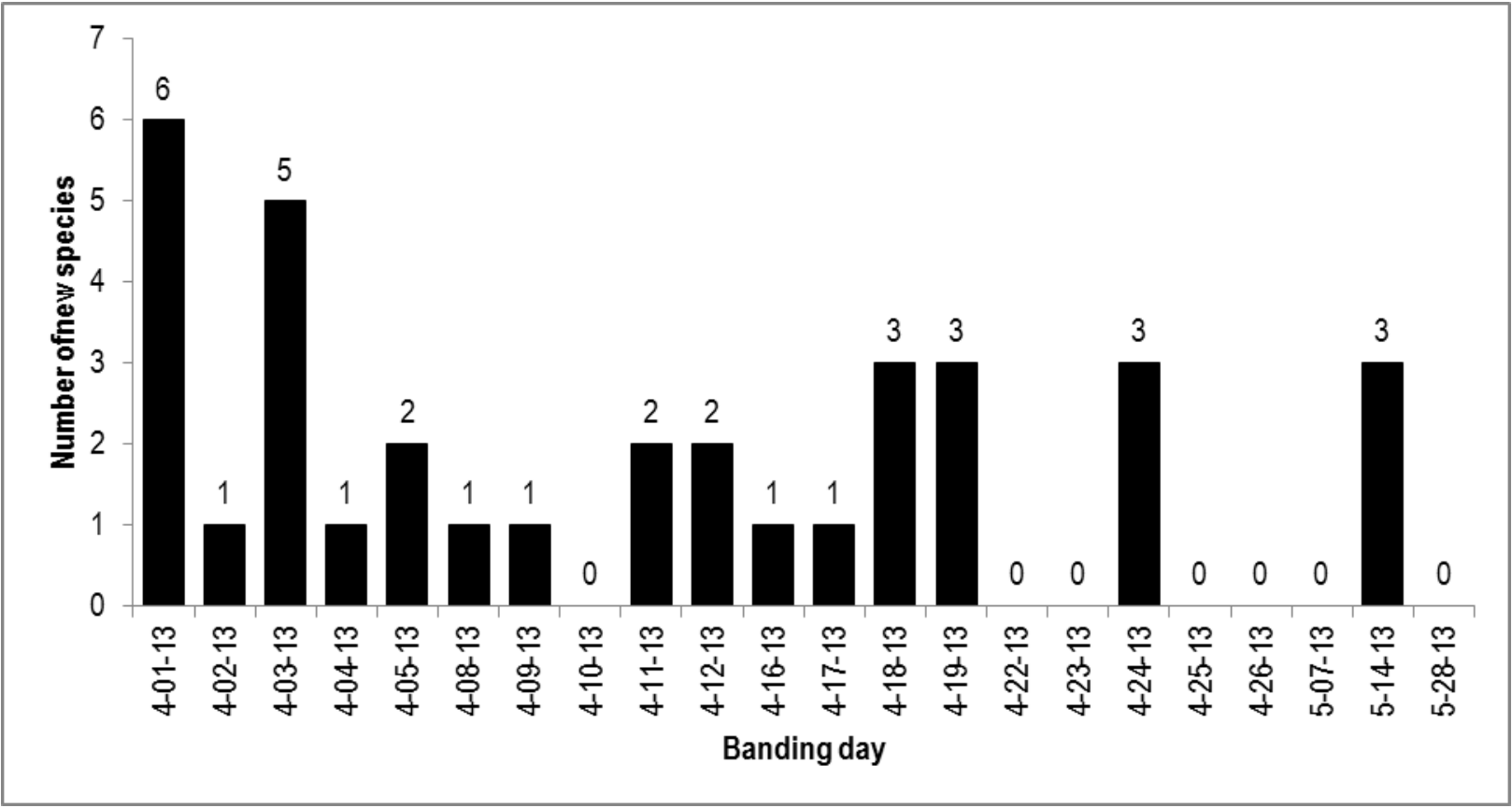

Figure B8. Number of new migrant species arriving per banding day during spring migration, Point Loma banding station, San Diego, California, 2013. 


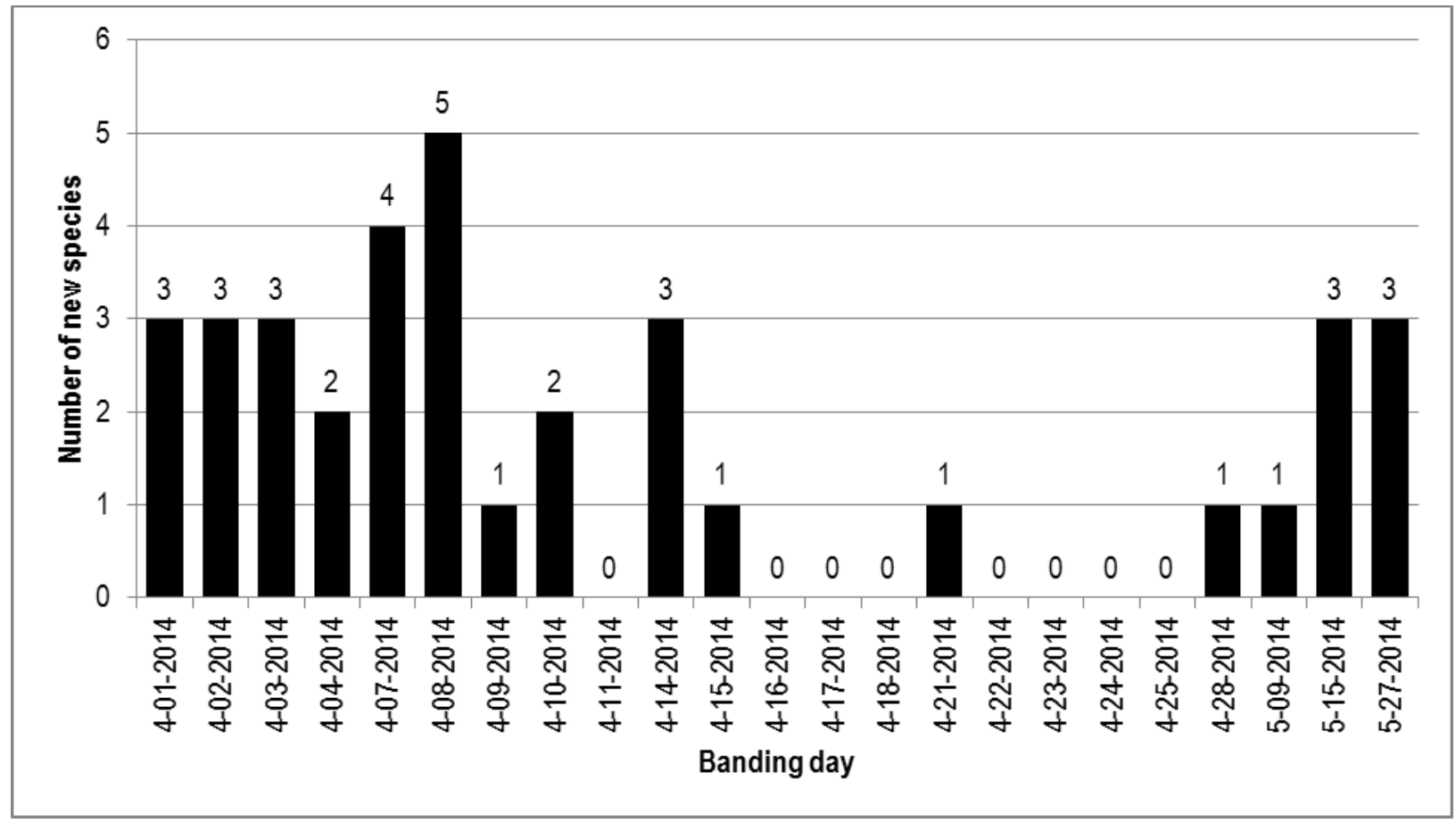

Figure B9. Number of new migrant species arriving per banding day during spring migration, Point Loma banding station, San Diego, California, 2014.

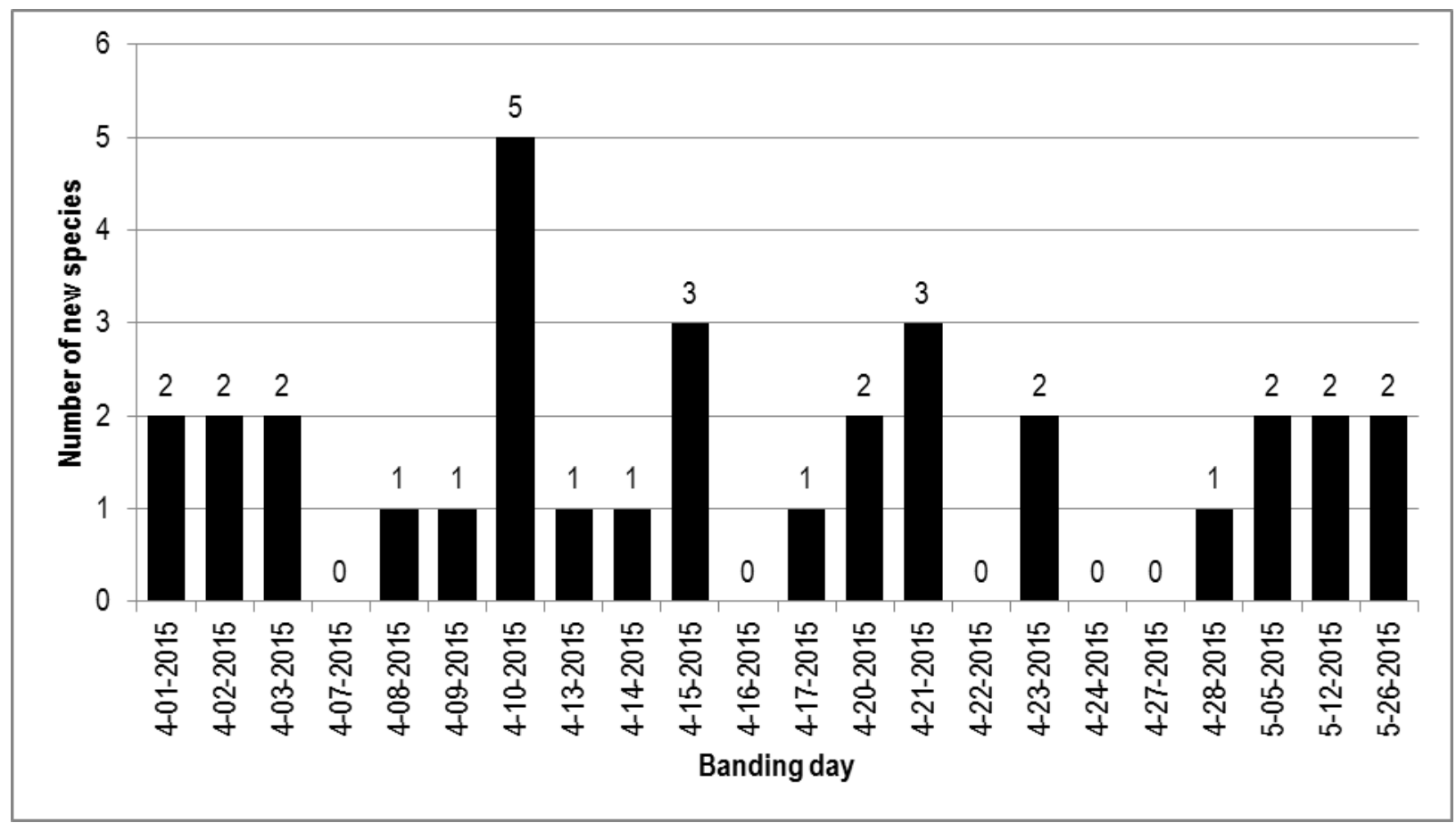

Figure B10. Number of new migrant species arriving per banding day during spring migration, Point Loma banding station, San Diego, California, 2015. 


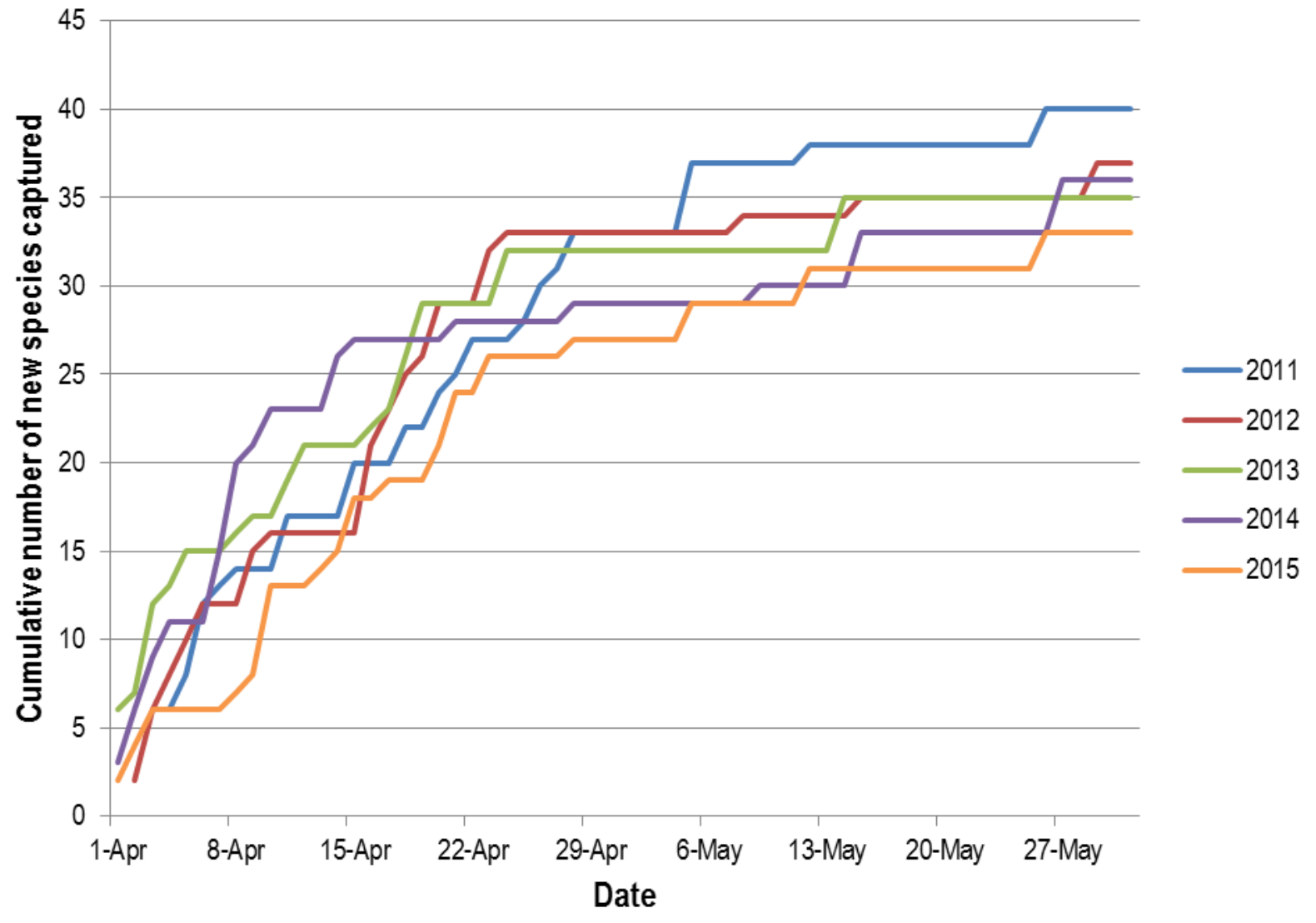

Figure B11. Cumulative number of new migrant species captured each banding day during spring migration, Point Loma banding station, San Diego, California, 2011-15. 


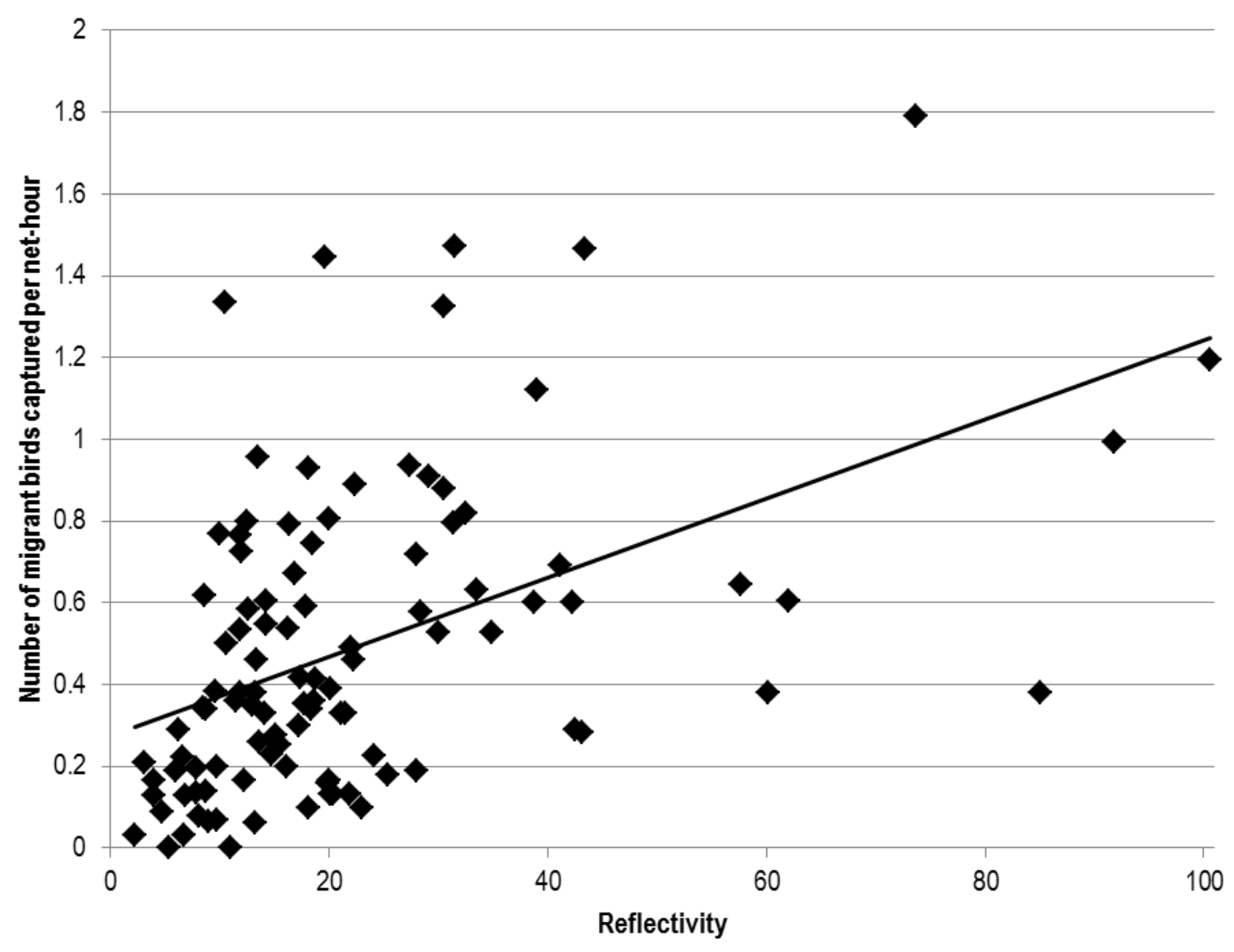

Figure B12. Correlation of the number of migrant birds captured per net-hour from 2011-15 with the reflectivity (Z) of the NEXRAD image from the evening before the netting day, Point Loma banding station, San Diego, California. Reflectivity is calculated as the sum of $Z^{\star}$ pixel area within $100 \mathrm{~km}$ of the San Diego weather station (KNKX). 


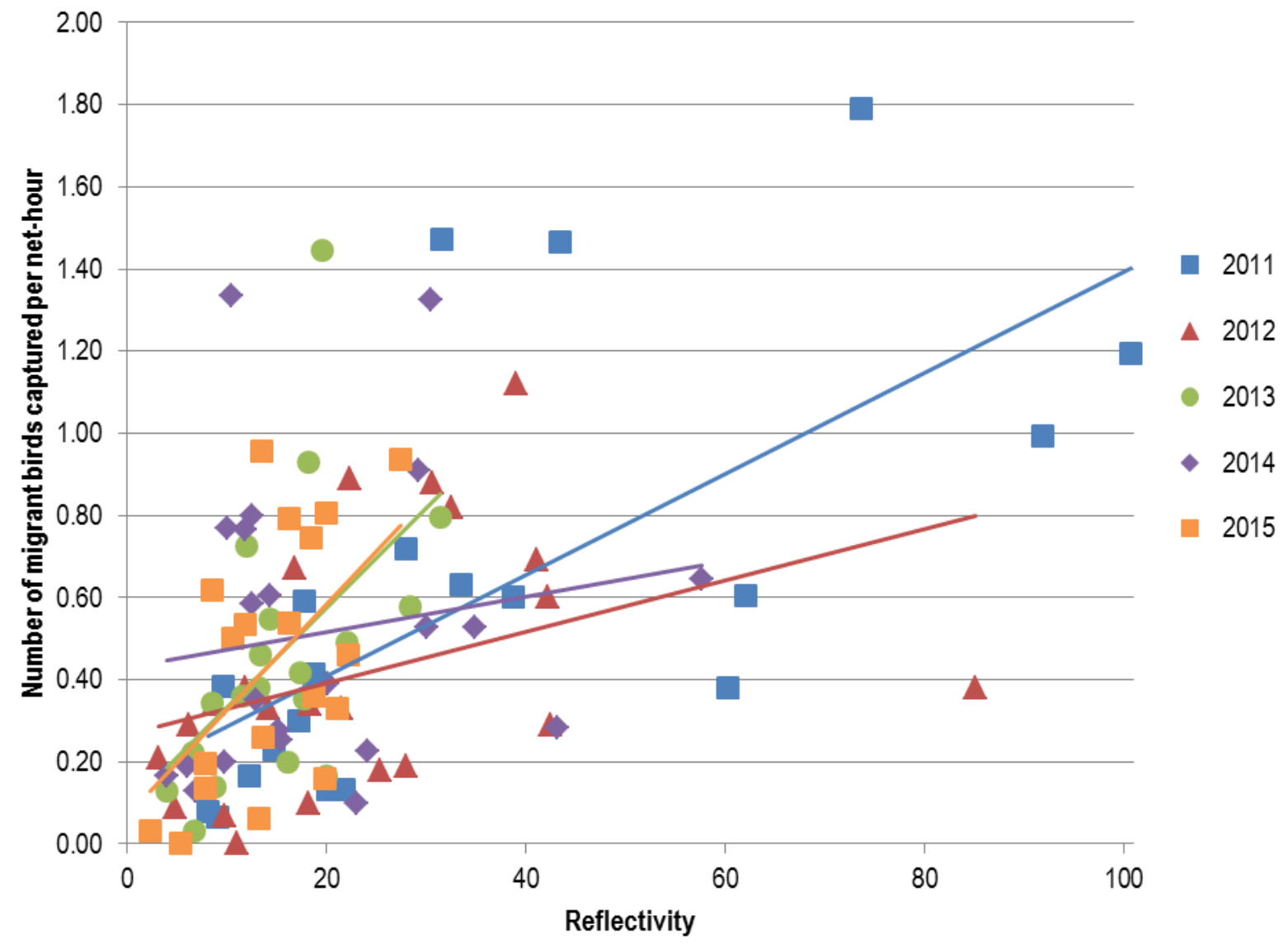

Figure B13. Correlation of the number of migrant birds captured per net-hour for each year from 2011-15 with the reflectivity $(Z)$ of the NEXRAD image from the evening before the netting day, Point Loma banding station, San Diego, California. Reflectivity is calculated as the sum of Z* pixel area within $100 \mathrm{~km}$ of the San Diego weather station (KNKX). 
Appendix C. Monitoring Avian Productivity and Survivorship Program, Point Loma, California, 2011-15 
Table C1. Number of birds captured and banded during Monitoring Avian Productivity and Survivorship, Point Loma banding station, San Diego, California, 2011-15.

[See appendix A for bird species codes. Species in italics are non-breeding neotropical migrants. Includes multiple captures of some individuals]

\begin{tabular}{|c|c|c|c|c|c|c|c|c|c|c|c|c|c|c|c|c|c|c|}
\hline \multirow{3}{*}{$\begin{array}{c}\text { Species } \\
\text { code }\end{array}$} & \multicolumn{6}{|c|}{ Total captures } & \multicolumn{6}{|c|}{ Total number of individuals captured } & \multicolumn{6}{|c|}{ New individuals banded } \\
\hline & \multicolumn{5}{|c|}{ Year } & \multirow[b]{2}{*}{ Total } & \multicolumn{5}{|c|}{ Year } & \multirow[b]{2}{*}{ Total } & \multicolumn{5}{|c|}{ Year } & \multirow[b]{2}{*}{ Total } \\
\hline & 2011 & 2012 & 2013 & 2014 & 2015 & & 2011 & 2012 & 2013 & 2014 & 2015 & & 2011 & 2012 & 2013 & 2014 & 2015 & \\
\hline AMKE & 0 & 0 & 0 & 0 & 1 & 1 & 0 & 0 & 0 & 0 & 1 & 1 & 0 & 0 & 0 & 0 & 0 & 0 \\
\hline CAQU & 1 & 0 & 0 & 0 & 0 & 1 & 1 & 0 & 0 & 0 & 0 & 1 & 0 & 0 & 0 & 0 & 0 & 0 \\
\hline MODO & 0 & 0 & 0 & 0 & 1 & 1 & 0 & 0 & 0 & 0 & 1 & 1 & 0 & 0 & 0 & 0 & 0 & 0 \\
\hline $\mathrm{BCHU}$ & 0 & 2 & 0 & 1 & 0 & 3 & 0 & 2 & 0 & 1 & 0 & 3 & 0 & 0 & 0 & 0 & 0 & 0 \\
\hline ANHU & 55 & 46 & 46 & 56 & 8 & 211 & 55 & 46 & 46 & 56 & 8 & 211 & 0 & 0 & 0 & 0 & 0 & 0 \\
\hline $\mathrm{COHU}$ & 5 & 1 & 2 & 8 & 3 & 19 & 5 & 1 & 2 & 8 & 3 & 19 & 0 & 0 & 0 & 0 & 0 & 0 \\
\hline CAHU & 0 & 1 & 0 & 2 & 2 & 5 & 0 & 1 & 0 & 2 & 2 & 5 & 0 & 0 & 0 & 0 & 0 & 0 \\
\hline RUHU & 1 & 4 & 1 & 0 & 0 & 6 & 1 & 4 & 1 & 0 & 0 & 6 & 0 & 0 & 0 & 0 & 0 & 0 \\
\hline$A L H U$ & 2 & 2 & 7 & 11 & 3 & 25 & 2 & 2 & 7 & 11 & 3 & 25 & 0 & 0 & 0 & 0 & 0 & 0 \\
\hline USHU & 1 & 2 & 1 & 1 & 0 & 5 & 1 & 2 & 1 & 1 & 0 & 5 & 0 & 0 & 0 & 0 & 0 & 0 \\
\hline UNHU & 4 & 0 & 0 & 0 & 0 & 4 & 4 & 0 & 0 & 0 & 0 & 4 & 0 & 0 & 0 & 0 & 0 & 0 \\
\hline DOWO & 0 & 0 & 0 & 1 & 0 & 1 & 0 & 0 & 0 & 1 & 0 & 1 & 0 & 0 & 0 & 1 & 0 & 1 \\
\hline WEWP & 3 & 5 & 2 & 1 & 5 & 16 & 3 & 5 & 2 & 1 & 5 & 16 & 3 & 5 & 2 & 1 & 5 & 16 \\
\hline WIFL & 2 & 3 & 2 & 2 & 3 & 12 & 2 & 3 & 2 & 2 & 3 & 12 & 2 & 3 & 2 & 2 & 3 & 12 \\
\hline HAFL & 0 & 0 & 1 & 2 & 1 & 4 & 0 & 0 & 1 & 2 & 1 & 4 & 0 & 0 & 1 & 2 & 1 & 4 \\
\hline GRFL & 0 & 0 & 1 & 0 & 0 & 1 & 0 & 0 & 1 & 0 & 0 & 1 & 0 & 0 & 1 & 0 & 0 & 1 \\
\hline DUFL & 1 & 0 & 0 & 0 & 0 & 1 & 1 & 0 & 0 & 0 & 0 & 1 & 1 & 0 & 0 & 0 & 0 & 1 \\
\hline PSFL & 12 & 32 & 13 & 74 & 31 & 162 & 12 & 32 & 13 & 74 & 31 & 162 & 11 & 32 & 13 & 74 & 30 & 160 \\
\hline COFL & 1 & 0 & 1 & 0 & 0 & 2 & 1 & 0 & 1 & 0 & 0 & 2 & 1 & 0 & 1 & 0 & 0 & 2 \\
\hline ATFL & 4 & 5 & 3 & 3 & 2 & 17 & 3 & 5 & 3 & 3 & 2 & 16 & 3 & 5 & 3 & 3 & 2 & 16 \\
\hline CAKI & 0 & 0 & 0 & 0 & 1 & 1 & 0 & 0 & 0 & 0 & 1 & 1 & 0 & 0 & 0 & 0 & 1 & 1 \\
\hline LBVI & 0 & 0 & 2 & 1 & 0 & 3 & 0 & 0 & 2 & 1 & 0 & 3 & 0 & 0 & 2 & 1 & 0 & 3 \\
\hline CAVI & 0 & 0 & 1 & 0 & 0 & 1 & 0 & 0 & 1 & 0 & 0 & 1 & 0 & 0 & 1 & 0 & 0 & 1 \\
\hline WAVI & 23 & 16 & 7 & 31 & 26 & 103 & 22 & 16 & 7 & 31 & 25 & 101 & 22 & 16 & 7 & 31 & 25 & 101 \\
\hline REVI & 0 & 0 & 1 & 0 & 0 & 1 & 0 & 0 & 1 & 0 & 0 & 1 & 0 & 0 & 1 & 0 & 0 & 1 \\
\hline
\end{tabular}




\begin{tabular}{|c|c|c|c|c|c|c|c|c|c|c|c|c|c|c|c|c|c|c|}
\hline \multirow{3}{*}{$\begin{array}{c}\text { Species } \\
\text { code }\end{array}$} & \multicolumn{6}{|c|}{ Total captures } & \multicolumn{6}{|c|}{ Total number of individuals captured } & \multicolumn{6}{|c|}{ New individuals banded } \\
\hline & \multicolumn{5}{|c|}{ Year } & \multirow[b]{2}{*}{ Total } & \multicolumn{5}{|c|}{ Year } & \multirow[b]{2}{*}{ Total } & \multicolumn{5}{|c|}{ Year } & \multirow[b]{2}{*}{ Total } \\
\hline & 2011 & 2012 & 2013 & 2014 & 2015 & & 2011 & 2012 & 2013 & 2014 & 2015 & & 2011 & 2012 & 2013 & 2014 & 2015 & \\
\hline WESJ & 1 & 2 & 0 & 0 & 0 & 3 & 1 & 2 & 0 & 0 & 0 & 3 & 1 & 2 & 0 & 0 & 0 & 3 \\
\hline BARS & 0 & 0 & 0 & 0 & 1 & 1 & 0 & 0 & 0 & 0 & 1 & 1 & 0 & 0 & 0 & 0 & 1 & 1 \\
\hline $\mathrm{BUSH}$ & 53 & 52 & 39 & 11 & 18 & 173 & 46 & 47 & 33 & 10 & 18 & 154 & 34 & 35 & 29 & 5 & 14 & 117 \\
\hline BEWR & 18 & 14 & 18 & 10 & 35 & 95 & 16 & 14 & 15 & 10 & 31 & 86 & 14 & 9 & 12 & 4 & 25 & 64 \\
\hline HOWR & 2 & 0 & 2 & 0 & 0 & 4 & 2 & 0 & 2 & 0 & 0 & 4 & 2 & 0 & 2 & 0 & 0 & 4 \\
\hline$R C K I$ & 0 & 0 & 0 & 0 & 1 & 1 & 0 & 0 & 0 & 0 & 1 & 1 & 0 & 0 & 0 & 0 & 1 & 1 \\
\hline CAGN & 0 & 0 & 1 & 0 & 1 & 2 & 0 & 0 & 1 & 0 & 1 & 2 & 0 & 0 & 1 & 0 & 1 & 2 \\
\hline SWTH & 8 & 4 & 2 & 8 & 0 & 22 & 8 & 4 & 2 & 8 & 0 & 22 & 8 & 4 & 2 & 8 & 0 & 22 \\
\hline HETH & 1 & 0 & 0 & 1 & 0 & 2 & 1 & 0 & 0 & 1 & 0 & 2 & 1 & 0 & 0 & 1 & 0 & 2 \\
\hline WREN & 20 & 8 & 9 & 15 & 28 & 80 & 20 & 8 & 9 & 14 & 25 & 76 & 17 & 6 & 8 & 8 & 21 & 60 \\
\hline GRCA & 0 & 0 & 0 & 0 & 1 & 1 & 0 & 0 & 0 & 0 & 1 & 1 & 0 & 0 & 0 & 0 & 1 & 1 \\
\hline NOMO & 3 & 0 & 1 & 1 & 1 & 6 & 3 & 0 & 1 & 1 & 1 & 6 & 2 & 0 & 1 & 1 & 1 & 5 \\
\hline CATH & 12 & 5 & 3 & 2 & 7 & 29 & 12 & 5 & 3 & 2 & 7 & 29 & 12 & 4 & 1 & 1 & 6 & 24 \\
\hline PHAI & 1 & 0 & 0 & 0 & 0 & 1 & 1 & 0 & 0 & 0 & 0 & 1 & 1 & 0 & 0 & 0 & 0 & 1 \\
\hline OCWA & 80 & 27 & 29 & 12 & 19 & 167 & 73 & 26 & 29 & 12 & 18 & 158 & 56 & 16 & 17 & 10 & 10 & 109 \\
\hline YEWA & 3 & 0 & 0 & 5 & 5 & 13 & 3 & 0 & 0 & 5 & 5 & 13 & 3 & 0 & 0 & 5 & 5 & 13 \\
\hline AUWA & 0 & 1 & 0 & 0 & 0 & 1 & 0 & 1 & 0 & 0 & 0 & 1 & 0 & 1 & 0 & 0 & 0 & 1 \\
\hline$B T Y W$ & 1 & 0 & 0 & 0 & 0 & 1 & 1 & 0 & 0 & 0 & 0 & 1 & 1 & 0 & 0 & 0 & 0 & 1 \\
\hline TOWA & 13 & 7 & 1 & 8 & 5 & 34 & 13 & 7 & 1 & 8 & 5 & 34 & 13 & 7 & 1 & 8 & 5 & 34 \\
\hline $\mathrm{THWH}$ & 2 & 0 & 0 & 0 & 0 & 2 & 2 & 0 & 0 & 0 & 0 & 2 & 2 & 0 & 0 & 0 & 0 & 2 \\
\hline HEWA & 2 & 4 & 2 & 3 & 1 & 12 & 2 & 4 & 2 & 3 & 1 & 12 & 2 & 4 & 2 & 2 & 1 & 11 \\
\hline MGWA & 1 & 0 & 1 & 7 & 0 & 9 & 1 & 0 & 1 & 7 & 0 & 9 & 1 & 0 & 1 & 7 & 0 & 9 \\
\hline COYE & 0 & 1 & 0 & 0 & 0 & 1 & 0 & 1 & 0 & 0 & 0 & 1 & 0 & 1 & 0 & 0 & 0 & 1 \\
\hline WIWA & 43 & 44 & 6 & 27 & 31 & 151 & 43 & 44 & 6 & 27 & 31 & 151 & 43 & 44 & 6 & 26 & 31 & 150 \\
\hline CAWA & 0 & 0 & 0 & 0 & 1 & 1 & 0 & 0 & 0 & 0 & 1 & 1 & 0 & 0 & 0 & 0 & 1 & 1 \\
\hline $\mathrm{YBCH}$ & 1 & 0 & 0 & 0 & 0 & 1 & 1 & 0 & 0 & 0 & 0 & 1 & 1 & 0 & 0 & 0 & 0 & 1 \\
\hline WETA & 3 & 2 & 3 & 3 & 3 & 14 & 3 & 2 & 3 & 3 & 3 & 14 & 3 & 2 & 3 & 3 & 3 & 14 \\
\hline SPTO & 27 & 13 & 28 & 12 & 27 & 107 & 27 & 12 & 26 & 12 & 25 & 102 & 21 & 6 & 18 & 8 & 20 & 73 \\
\hline CALT & 34 & 10 & 8 & 11 & 22 & 85 & 30 & 9 & 8 & 10 & 20 & 77 & 26 & 6 & 5 & 5 & 15 & 57 \\
\hline CHSP & 2 & 0 & 0 & 0 & 0 & 2 & 2 & 0 & 0 & 0 & 0 & 2 & 1 & 0 & 0 & 0 & 0 & 1 \\
\hline SAGS & 0 & 0 & 0 & 1 & 0 & 1 & 0 & 0 & 0 & 1 & 0 & 1 & 0 & 0 & 0 & 1 & 0 & 1 \\
\hline
\end{tabular}




\begin{tabular}{|c|c|c|c|c|c|c|c|c|c|c|c|c|c|c|c|c|c|c|}
\hline \multirow{3}{*}{$\begin{array}{c}\text { Species } \\
\text { code }\end{array}$} & \multicolumn{6}{|c|}{ Total captures } & \multicolumn{6}{|c|}{ Total number of individuals captured } & \multicolumn{6}{|c|}{ New individuals banded } \\
\hline & \multicolumn{5}{|c|}{ Year } & \multirow[b]{2}{*}{ Total } & \multicolumn{5}{|c|}{ Year } & \multirow[b]{2}{*}{ Total } & \multicolumn{5}{|c|}{ Year } & \multirow[b]{2}{*}{ Total } \\
\hline & 2011 & 2012 & 2013 & 2014 & 2015 & & 2011 & 2012 & 2013 & 2014 & 2015 & & 2011 & 2012 & 2013 & 2014 & 2015 & \\
\hline SOSP & 1 & 4 & 0 & 0 & 0 & 5 & 1 & 4 & 0 & 0 & 0 & 5 & 0 & 4 & 0 & 0 & 0 & 4 \\
\hline WTSP & 1 & 0 & 0 & 0 & 0 & 1 & 1 & 0 & 0 & 0 & 0 & 1 & 1 & 0 & 0 & 0 & 0 & 1 \\
\hline ORJU & 0 & 0 & 0 & 1 & 0 & 1 & 0 & 0 & 0 & 1 & 0 & 1 & 0 & 0 & 0 & 1 & 0 & 1 \\
\hline BHGR & 1 & 0 & 2 & 0 & 0 & 3 & 1 & 0 & 2 & 0 & 0 & 3 & 0 & 0 & 2 & 0 & 0 & 2 \\
\hline$B L G R$ & 1 & 1 & 0 & 0 & 0 & 2 & 1 & 1 & 0 & 0 & 0 & 2 & 1 & 1 & 0 & 0 & 0 & 2 \\
\hline LAZB & 3 & 2 & 0 & 0 & 1 & 6 & 3 & 2 & 0 & 0 & 1 & 6 & 3 & 2 & 0 & 0 & 1 & 6 \\
\hline INBU & 0 & 1 & 0 & 0 & 0 & 1 & 0 & 1 & 0 & 0 & 0 & 1 & 0 & 1 & 0 & 0 & 0 & 1 \\
\hline OROR & 1 & 0 & 0 & 0 & 0 & 1 & 1 & 0 & 0 & 0 & 0 & 1 & 1 & 0 & 0 & 0 & 0 & 1 \\
\hline HOOR & 13 & 10 & 8 & 5 & 3 & 39 & 13 & 10 & 8 & 5 & 3 & 39 & 13 & 10 & 8 & 5 & 2 & 38 \\
\hline BUOR & 2 & 0 & 0 & 0 & 0 & 2 & 2 & 0 & 0 & 0 & 0 & 2 & 2 & 0 & 0 & 0 & 0 & 2 \\
\hline $\mathrm{HOFI}$ & 8 & 3 & 11 & 4 & 1 & 27 & 8 & 3 & 11 & 4 & 1 & 27 & 7 & 3 & 11 & 4 & 1 & 26 \\
\hline LEGO & 12 & 0 & 2 & 0 & 6 & 20 & 12 & 0 & 2 & 0 & 6 & 20 & 12 & 0 & 2 & 0 & 6 & 20 \\
\hline AMGO & 1 & 0 & 0 & 0 & 0 & 1 & 1 & 0 & 0 & 0 & 0 & 1 & 1 & 0 & 0 & 0 & 0 & 1 \\
\hline Total & 490 & 334 & 267 & 341 & 305 & 1737 & 468 & 326 & 256 & 338 & 292 & 1680 & 349 & 229 & 166 & 228 & 239 & 1211 \\
\hline
\end{tabular}


Table C2. Number of banded birds recaptured during Monitoring Avian Productivity and Survivorship, Point Loma banding station, San Diego, California, 2011-15.

[See appendix A for bird species codes. Species in italics are non-breeding neotropical migrants. Includes multiple captures of some individuals. Recaptures originally banded during previous banding activities at Point Loma, 2006-10 (Institute for Bird Populations, unpub. data, 2006-2010, or from previous USGS banding activities, 2010-14, including original captures during spring 2015]

\begin{tabular}{|c|c|c|c|c|c|c|c|c|c|c|c|c|c|c|c|c|c|c|c|c|c|c|c|c|c|c|c|c|c|c|c|c|c|c|c|c|}
\hline \multirow{3}{*}{$\begin{array}{c}\text { Species } \\
\text { code }\end{array}$} & \multicolumn{7}{|c|}{ Recaptured individuals, 2011} & \multicolumn{7}{|c|}{ Recaptured individuals, 2012} & \multicolumn{9}{|c|}{ Recaptured individuals, 2013} & \multicolumn{6}{|c|}{ Recaptured individuals, 2014} & \multicolumn{7}{|c|}{ Recaptured individuals, 2015} \\
\hline & \multicolumn{6}{|c|}{ Year originally banded } & \multirow[b]{2}{*}{ Total } & \multicolumn{6}{|c|}{ Year originally banded } & \multirow[b]{2}{*}{ Total } & \multicolumn{8}{|c|}{ Year originally banded } & \multirow[b]{2}{*}{ Total } & \multicolumn{5}{|c|}{ Year originally banded } & & \multicolumn{6}{|c|}{ Year originally banded } & \multirow[b]{2}{*}{ Total } \\
\hline & 2006 & 2007 & 2008 & 2009 & 2010 & 2011 & & 20062 & 20082 & 2009 & 2010 & \begin{tabular}{|l|l|l|}
2011 & 2 \\
\end{tabular} & \begin{tabular}{|l|l}
2012 & $T$ \\
\end{tabular} & & 2006 & 2007 & 20082 & 20092 & 2010 & 2011 & \begin{tabular}{l|l}
2012 & 2 \\
\end{tabular} & 2013 & & 2010 & 2011 & 2012 & 20132 & $2014 \mathrm{Tc}$ & Total & 2010 & 20112 & 2012 & 2013 & 2014 & $2015 \mathrm{~T}$ & \\
\hline AMKE & 0 & 0 & 0 & 0 & 0 & 0 & 0 & 0 & 0 & 0 & 0 & 0 & 0 & 0 & 0 & 0 & 0 & 0 & 0 & 0 & 0 & 0 & 0 & 0 & 0 & 0 & 0 & 0 & 0 & 0 & 0 & 0 & 0 & 0 & 0 & 0 \\
\hline CAQU & 0 & 0 & 0 & 0 & 0 & 0 & 0 & 0 & 0 & 0 & 0 & 0 & 0 & 0 & 0 & 0 & 0 & 0 & 0 & 0 & 0 & 0 & 0 & 0 & 0 & 0 & 0 & 0 & 0 & 0 & 0 & 0 & 0 & 0 & 0 & 0 \\
\hline MODO & 0 & 0 & 0 & 0 & 0 & 0 & 0 & 0 & 0 & 0 & 0 & 0 & 0 & 0 & 0 & 0 & 0 & 0 & 0 & 0 & 0 & 0 & 0 & 0 & 0 & 0 & 0 & 0 & 0 & 0 & 0 & 0 & 0 & 0 & 0 & 0 \\
\hline $\mathrm{BCHU}$ & 0 & 0 & 0 & 0 & 0 & 0 & 0 & 0 & 0 & 0 & 0 & 0 & 0 & 0 & 0 & 0 & 0 & 0 & 0 & 0 & 0 & 0 & 0 & 0 & 0 & 0 & 0 & 0 & 0 & 0 & 0 & 0 & 0 & 0 & 0 & 0 \\
\hline ANHU & 0 & 0 & 0 & 0 & 0 & 0 & 0 & 0 & 0 & 0 & 0 & 0 & 0 & 0 & 0 & 0 & 0 & 0 & 0 & 0 & 0 & 0 & 0 & 0 & 0 & 0 & 0 & 0 & 0 & 0 & 0 & 0 & 0 & 0 & 0 & 0 \\
\hline $\mathrm{COHU}$ & 0 & 0 & 0 & 0 & 0 & 0 & 0 & 0 & 0 & 0 & 0 & 0 & 0 & 0 & 0 & 0 & 0 & 0 & 0 & 0 & 0 & 0 & 0 & 0 & 0 & 0 & 0 & 0 & 0 & 0 & 0 & 0 & 0 & 0 & 0 & 0 \\
\hline CAHU & 0 & 0 & 0 & 0 & 0 & 0 & 0 & 0 & 0 & 0 & 0 & 0 & 0 & 0 & 0 & 0 & 0 & 0 & 0 & 0 & 0 & 0 & 0 & 0 & 0 & 0 & 0 & 0 & 0 & 0 & 0 & 0 & 0 & 0 & 0 & 0 \\
\hline RUHU & 0 & 0 & 0 & 0 & 0 & 0 & 0 & 0 & 0 & 0 & 0 & 0 & 0 & 0 & 0 & 0 & 0 & 0 & 0 & 0 & 0 & 0 & 0 & 0 & 0 & 0 & 0 & 0 & 0 & 0 & 0 & 0 & 0 & 0 & 0 & 0 \\
\hline ALHU & 0 & 0 & 0 & 0 & 0 & 0 & 0 & 0 & 0 & 0 & 0 & 0 & 0 & 0 & 0 & 0 & 0 & 0 & 0 & 0 & 0 & 0 & 0 & 0 & 0 & 0 & 0 & 0 & 0 & 0 & 0 & 0 & 0 & 0 & 0 & 0 \\
\hline USHU & 0 & 0 & 0 & 0 & 0 & 0 & 0 & 0 & 0 & 0 & 0 & 0 & 0 & 0 & 0 & 0 & 0 & 0 & 0 & 0 & 0 & 0 & 0 & 0 & 0 & 0 & 0 & 0 & 0 & 0 & 0 & 0 & 0 & 0 & 0 & 0 \\
\hline UNHU & 0 & 0 & 0 & 0 & 0 & 0 & 0 & 0 & 0 & 0 & 0 & 0 & 0 & 0 & 0 & 0 & 0 & 0 & 0 & 0 & 0 & 0 & 0 & 0 & 0 & 0 & 0 & 0 & 0 & 0 & 0 & 0 & 0 & 0 & 0 & 0 \\
\hline DOWO & 0 & 0 & 0 & 0 & 0 & 0 & 0 & 0 & 0 & 0 & 0 & 0 & 0 & 0 & 0 & 0 & 0 & 0 & 0 & 0 & 0 & 0 & 0 & 0 & 0 & 0 & 0 & 0 & 0 & 0 & 0 & 0 & 0 & 0 & 0 & 0 \\
\hline WEWP & 0 & 0 & 0 & 0 & 0 & 0 & 0 & 0 & 0 & 0 & 0 & 0 & 0 & 0 & 0 & 0 & 0 & 0 & 0 & 0 & 0 & 0 & 0 & 0 & 0 & 0 & 0 & 0 & 0 & 0 & 0 & 0 & 0 & 0 & 0 & 0 \\
\hline WIFL & 0 & 0 & 0 & 0 & 0 & 0 & 0 & 0 & 0 & 0 & 0 & 0 & 0 & 0 & 0 & 0 & 0 & 0 & 0 & 0 & 0 & 0 & 0 & 0 & 0 & 0 & 0 & 0 & 0 & 0 & 0 & 0 & 0 & 0 & 0 & 0 \\
\hline HAFL & 0 & 0 & 0 & 0 & 0 & 0 & 0 & 0 & 0 & 0 & 0 & 0 & 0 & 0 & 0 & 0 & 0 & 0 & 0 & 0 & 0 & 0 & 0 & 0 & 0 & 0 & 0 & 0 & 0 & 0 & 0 & 0 & 0 & 0 & 0 & 0 \\
\hline GRFL & 0 & 0 & 0 & 0 & 0 & 0 & 0 & 0 & 0 & 0 & 0 & 0 & 0 & 0 & 0 & 0 & 0 & 0 & 0 & 0 & 0 & 0 & 0 & 0 & 0 & 0 & 0 & 0 & 0 & 0 & 0 & 0 & 0 & 0 & 0 & 0 \\
\hline DUFL & 0 & 0 & 0 & 0 & 0 & 0 & 0 & 0 & 0 & 0 & 0 & 0 & 0 & 0 & 0 & 0 & 0 & 0 & 0 & 0 & 0 & 0 & 0 & 0 & 0 & 0 & 0 & 0 & 0 & 0 & 0 & 0 & 0 & 0 & 0 & 0 \\
\hline PSFL & 0 & 0 & 0 & 0 & 0 & 0 & 0 & 0 & 0 & 0 & 0 & 0 & 0 & 0 & 0 & 0 & 0 & 0 & 0 & 0 & 0 & 0 & 0 & 0 & 0 & 0 & 0 & 0 & 0 & 0 & 0 & 0 & 0 & 0 & 0 & 0 \\
\hline COFL & 0 & 0 & 0 & 0 & 0 & 0 & 0 & 0 & 0 & 0 & 0 & 0 & 0 & 0 & 0 & 0 & 0 & 0 & 0 & 0 & 0 & 0 & 0 & 0 & 0 & 0 & 0 & 0 & 0 & 0 & 0 & 0 & 0 & 0 & 0 & 0 \\
\hline ATFL & 0 & 0 & 0 & 0 & 0 & 0 & 0 & 0 & 0 & 0 & 0 & 0 & 0 & 0 & 0 & 0 & 0 & 0 & 0 & 0 & 0 & 0 & 0 & 0 & 0 & 0 & 0 & 0 & 0 & 0 & 0 & 0 & 0 & 0 & 0 & 0 \\
\hline CAKI & 0 & 0 & 0 & 0 & 0 & 0 & 0 & 0 & 0 & 0 & 0 & 0 & 0 & 0 & 0 & 0 & 0 & 0 & 0 & 0 & 0 & 0 & 0 & 0 & 0 & 0 & 0 & 0 & 0 & 0 & 0 & 0 & 0 & 0 & 0 & 0 \\
\hline LBVI & 0 & 0 & 0 & 0 & 0 & 0 & 0 & 0 & 0 & 0 & 0 & 0 & 0 & 0 & 0 & 0 & 0 & 0 & 0 & 0 & 0 & 0 & 0 & 0 & 0 & 0 & 0 & 0 & 0 & 0 & 0 & 0 & 0 & 0 & 0 & 0 \\
\hline
\end{tabular}




\begin{tabular}{|c|c|c|c|c|c|c|c|c|c|c|c|c|c|c|c|c|c|c|c|c|c|c|c|c|c|c|c|c|c|c|c|c|c|c|c|c|c|c|c|c|c|c|}
\hline \multirow{3}{*}{$\begin{array}{c}\text { Species } \\
\text { code }\end{array}$} & \multicolumn{8}{|c|}{ Recaptured individuals, 2011} & \multicolumn{8}{|c|}{ Recaptured individuals, 2012} & \multicolumn{11}{|c|}{ Recaptured individuals, 2013} & \multicolumn{7}{|c|}{ Recaptured individuals, 2014} & \multicolumn{8}{|c|}{ Recaptured individuals, 2015} \\
\hline & \multicolumn{7}{|c|}{ Year originally banded } & & \multicolumn{7}{|c|}{ Year originally banded } & \multirow[b]{2}{*}{ Total } & \multicolumn{10}{|c|}{ Year originally banded } & & \multicolumn{5}{|c|}{ Year originally banded } & & & & ear or & rigin & ally ba & anded & & & \\
\hline & 2006 & 2007 & & & 009 & 2010 & 2011 & Tot: & & 0062 & $20082_{2}$ & 2009 & 2010 & 2011 & 2012 & & 200 & \begin{tabular}{l|l}
006 & 20
\end{tabular} & 00720 & 20082 & 2009 & 2010 & 2011 & & 201 & & & 2010 & 2011 & 2012 & 2013 & & & & 2010 & 20112 & 2012 & 2013 & & & 5 Tota & \\
\hline CAVI & 0 & 0 & & 0 & 0 & 0 & 0 & 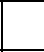 & & 0 & 0 & 0 & 0 & 0 & & & & 0 & 0 & 0 & 0 & 0 & 0 & $\begin{array}{lll}0 & 0 \\
\end{array}$ & 0 & 0 & & 0 & 0 & 0 & 0 & & & & 0 & 0 & & 0 & & $\begin{array}{lll}0 & 0 \\
\end{array}$ & & \\
\hline WAVI & & 0 & & 0 & 0 & 0 & 0 & & & 0 & 0 & & 0 & & & & & 0 & 0 & 0 & & 0 & 0 & 0 & & & & 0 & & 0 & 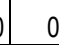 & & & & 0 & 0 & 0 & 0 & & 0 & & \\
\hline REVI & & 0 & & 0 & 0 & 0 & 0 & & & & 0 & 0 & 0 & & & & & 0 & 0 & 0 & 이 & 0 & 0 & 0 & 0 & 0 & & 0 & & t & & & & & 0 & 0 & 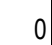 & 0 & & 0 & & \\
\hline WESJ & & 0 & & 0 & 0 & 0 & 0 & & & 0 & 0 & 0 & 0 & 0 & & & & 0 & 0 & 0 & 0 & 0 & & 0 & 0 & & & 0 & & 0 & & & & & 0 & 0 & & 0 & & 0 & & \\
\hline BARS & 0 & 0 & 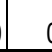 & 0 & 0 & 0 & 0 & & & 0 & 0 & 0 & 0 & . & & & & 0 & 0 & 0 & 0 & 0 & 0 & 0 & 0 & 0 & & 0 & . & 0 & 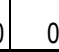 & 0 & & 이 & 0 & 0 & 0 & 0 & & 0 & & \\
\hline BUSH & & 0 & 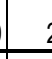 & 2 & 0 & $\underline{6}$ & $\underline{2}$ & 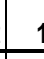 & & & 0 & 0 & 2 & 5 & & 1 & & 0 & 0 & 0 & 0 & 1 & $\underline{0}$ & $\underline{2}$ & 2 & 1 & & 0 & 0 & 0 & ) & & & & 0 & 0 & & 1 & 2 & 2 & & \\
\hline BEWR & 0 & 0 & 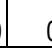 & 0 & 0 & 1 & 0 & & & 0 & 0 & 0 & 1 & 4 & & & & 0 & 1 & 1 & 0 & 0 & 0 & 0 & 0 & 0 & & 0 & 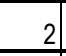 & 11 & & 0 & & 5 & 1 & 0 & 1 & 1 & 0 & 0 & & \\
\hline HOWR & & 0 & 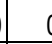 & 0 & 0 & 0 & 0 & & & 0 & 0 & ( & 0 & & & & & 0 & 0 & 0 & 0 & 0 & 0 & & 0 & & & 0 & & . & & & & & 0 & 0 & 证 & 0 & 0 & 0 & & \\
\hline RCKI & 0 & 0 & 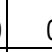 & 0 & 0 & 0 & 0 & & & 0 & 0 & 0 & 0 & 0 & & & & 0 & 0 & 0 & 0 & 0 & & 0 & 0 & 0 & & 0 & & 0 & ) & - & & $\theta$ & 0 & 0 & 0 & 0 & & 0 & & \\
\hline CAGN & 0 & 0 & 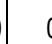 & 0 & 0 & 0 & 0 & & & 0 & 0 & 0 & 0 & 0 & & & & 0 & 0 & 0 & 0 & 0 & 0 & & 0 & 0 & & 0 & 0 & 0 & 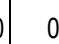 & 0 & & 0 & 0 & 0 & 0 & 0 & 0 & 0 & & \\
\hline SWTH & & 0 & 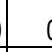 & 0 & 0 & 0 & 0 & & & & 0 & 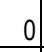 & 0 & & & & & 0 & 0 & 0 & & 0 & & 0 & 0 & & & 0 & & 0 & & & & & & & & 0 & & 0 & & \\
\hline HETH & 0 & 0 & b & 0 & 0 & 0 & 0 & & & 0 & 0 & 0 & 0 & 0 & c & & & 0 & 0 & 0 & 0 & 0 & & 0 & 0 & 0 & & 0 & 0 & 0 & ) & 0 & & 0 & 0 & 0 & 0 & 0 & 0 & 0 & & \\
\hline WREN & 0 & 1 & & 0 & 0 & 2 & 0 & & & 0 & 0 & 1 & 0 & 0 & & & & 1 & 0 & 0 & 0 & 0 & & & 0 & 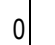 & & 1 & & & & & & 6 & 1 & 1 & 0 & 0 & 0 & 0 & & \\
\hline GRCA & 0 & 0 & D & 0 & 0 & 0 & 0 & & & 0 & 0 & 0 & 0 & 0 & & & & 0 & 0 & 0 & 0 & 0 & 0 & 0 & 0 & 0 & & 0 & 0 & 0 & ) & 0 & & & 0 & 0 & 0 & 0 & & 0 & & \\
\hline NOMO & 0 & 0 & b & 0 & 0 & 0 & 1 & & & 0 & 0 & 0 & 0 & 0 & 0 & & & 0 & 0 & 0 & 0 & 0 & & 0 & 0 & 0 & & 0 & 0 & 0 & ) & 0 & & 0 & 0 & 0 & 0 & 0 & 0 & 0 & & \\
\hline CATH & 0 & 0 & & 0 & 0 & 0 & 0 & & & 0 & 0 & 1 & 0 & 0 & & & & 1 & 0 & 0 & 0 & 0 & & 0 & 0 & 0 & & 0 & 0 & 1 & 1 & & & & 1 & 0 & 0 & 0 & 0 & 0 & & \\
\hline PHAI & 0 & 0 & D & 0 & 0 & 0 & 0 & & & 0 & 0 & 0 & 0 & 0 & 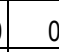 & & & 0 & 0 & 0 & 0 & 0 & 0 & 0 & 0 & 0 & & 0 & 0 & 0 & ) & 0 & & & 0 & 0 & 0 & 0 & 0 & 0 & & \\
\hline OCWA & 1 & 2 & & 0 & 3 & 3 & 7 & 1 & & 0 & 1 & 2 & 1 & 5 & 1 & 1 & & 0 & 0 & 0 & 1 & 3 & & 4 & 3 & 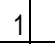 & 12 & 0 & 2 & 0 & & & & & 2 & 1 & & 0 & 0 & 0 & & \\
\hline YEWA & 0 & 0 & & 0 & 0 & 0 & 0 & & & 0 & 0 & 0 & 0 & 0 & 0 & & & 0 & 0 & 0 & 0 & 0 & 0 & 0 & 0 & 0 & 0 & 0 & 0 & 0 & 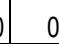 & 0 & & 0 & 0 & 0 & 0 & 0 & 0 & 0 & 0 & \\
\hline AUWA & 0 & 0 & b & 0 & 0 & 0 & 0 & & & 0 & 0 & 0 & 0 & 0 & & & & 0 & 0 & 0 & 0 & 0 & & 0 & 0 & 0 & & 0 & 0 & 0 & 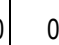 & 0 & & 0 & 0 & 0 & 0 & 0 & & 0 & & \\
\hline BTYW & 0 & 0 & & 0 & 0 & 0 & 0 & & & 0 & 0 & 0 & 0 & 0 & & & & 0 & 0 & 0 & 0 & 0 & & & 0 & 0 & & 0 & 0 & 0 & & 0 & & ? & 0 & 0 & 0 & 0 & 0 & 0 & & \\
\hline TOWA & 0 & 0 & & 0 & 0 & 0 & 0 & & & 0 & 0 & 0 & 0 & 0 & 0 & & & 0 & 0 & 0 & 0 & 0 & 0 & 0 & 0 & 0 & 0 & 0 & 0 & 0 & 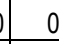 & 0 & 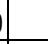 & 0 & 0 & 0 & 0 & 0 & 0 & 0 & 0 & \\
\hline THWH & 0 & 0 & b & 0 & 0 & 0 & 0 & & & 0 & 0 & 0 & 0 & 0 & & & & 0 & 0 & 0 & 0 & 0 & & 0 & 0 & 0 & 0 & 0 & 0 & 0 & ) & 0 & & 0 & 0 & 0 & 0 & 0 & 0 & 0 & & \\
\hline HEWA & 0 & 0 & b & 0 & 0 & 0 & 0 & & & 0 & 0 & 0 & 0 & 0 & 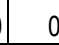 & & & 0 & 0 & 0 & 0 & 0 & 0 & 0 & 0 & 0 & 0 & 0 & 0 & 0 & 0 & 0 & 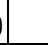 & 0 & 0 & 0 & 0 & 0 & 0 & 0 & & \\
\hline MGWA & 0 & 0 & D & 0 & 0 & 0 & $\underline{0}$ & & & 0 & 0 & 0 & 0 & 0 & & & & 0 & 0 & 0 & 0 & 0 & $\underline{0}$ & $\underline{0}$ & 0 & 0 & 0 & 0 & 0 & 0 & 0 & 0 & ) & 0 & 0 & 0 & 0 & 0 & 0 & 0 & 0 & \\
\hline COYE & 0 & 0 & D & 0 & 0 & 0 & 0 & & & 0 & 0 & 0 & 0 & 0 & 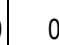 & & & 0 & 0 & 0 & 0 & 0 & 0 & 0 & 0 & 0 & 0 & 0 & 0 & 0 & ) & 0 & 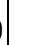 & 0 & 0 & 0 & 0 & 0 & 0 & 0 & 0 & \\
\hline WIWA & 0 & 0 & D & 0 & 0 & 0 & 0 & 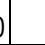 & & 0 & 0 & 0 & 0 & 0 & & & & 0 & 0 & 0 & 0 & 0 & 0 & 0 & 0 & 0 & & 0 & 0 & 0 & 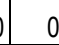 & 0 & 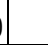 & 0 & 0 & 0 & 0 & 0 & & 0 & 0 & \\
\hline AWA & 0 & 0 & D & 0 & 0 & 0 & 0 & D & & 0 & 0 & 0 & 0 & & & & & 0 & 0 & 0 & 0 & 0 & & & 0 & & & 0 & & 0 & ) & 0 & & & 0 & 0 & 0 & 0 & 0 & 0 & 0 & \\
\hline
\end{tabular}




\begin{tabular}{|c|c|c|c|c|c|c|c|c|c|c|c|c|c|c|c|c|c|c|c|c|c|c|c|c|c|c|c|c|c|c|c|c|c|c|c|c|c|c|c|c|}
\hline \multirow{3}{*}{$\begin{array}{c}\text { Species } \\
\text { code }\end{array}$} & \multicolumn{8}{|c|}{ Recaptured individuals, 2011} & \multicolumn{7}{|c|}{ Recaptured individuals, 2012} & \multicolumn{10}{|c|}{ Recaptured individuals, 2013} & \multicolumn{7}{|c|}{ Recaptured individuals, 2014} & \multicolumn{8}{|c|}{ Recaptured individuals, 2015} \\
\hline & \multicolumn{7}{|c|}{ Year originally banded } & & \multicolumn{6}{|c|}{ Year originally banded } & \multirow[b]{2}{*}{ Total } & \multicolumn{9}{|c|}{ Year originally banded } & \multirow[b]{2}{*}{ Total } & \multicolumn{6}{|c|}{ Year originally banded } & & \multicolumn{7}{|c|}{ Year originally banded } & \multirow[b]{2}{*}{ Total } \\
\hline & 2006 & 2007 & 2008 & 2009 & 201 & & 0111 & Total & 2006 & 2008 & 2009 & 2010 & 2011 & 2012 & & 2006 & 2007 & 2008 & 2009 & 2010 & 201 & & 0122 & 2013 & & 2010 & 2011 & 201 & & 0132 & $2014 \mathrm{~T}$ & Total & 2010 & 201 & 1201 & 122 & 2013 & $2014 \sqrt{2}$ & 2015 & \\
\hline $\mathrm{YBCH}$ & 0 & 0 & 0 & 0 & & 0 & 0 & 0 & 0 & 0 & 0 & 0 & 0 & 0 & 0 & 0 & 0 & 0 & 0 & 0 & & 0 & 0 & 0 & 0 & 0 & 0 & & 0 & 0 & 0 & 0 & 0 & & 0 & 0 & 0 & 0 & 0 & 0 \\
\hline WETA & 0 & 0 & 0 & 0 & & 0 & 0 & 0 & 0 & 0 & 0 & 0 & 0 & 0 & 0 & 0 & 0 & 0 & 0 & 0 & & 0 & 0 & 0 & 0 & 0 & 0 & & 0 & 0 & 0 & 0 & 0 & & 0 & 0 & 0 & 0 & 0 & 0 \\
\hline SPTO & 0 & 0 & 1 & 0 & & 4 & 0 & 5 & 0 & 0 & 0 & 2 & 2 & 1 & 5 & 0 & 0 & 0 & 0 & 2 & & 2 & 2 & 2 & 8 & 0 & 1 & & 1 & 2 & 0 & 4 & 0 & & 0 & 0 & 0 & 2 & 2 & 4 \\
\hline CALT & 0 & 0 & 0 & 0 & & 3 & 1 & 4 & 0 & 0 & 0 & 0 & 2 & 1 & 3 & 0 & 0 & 0 & 0 & 0 & & 1 & 0 & 1 & 2 & 1 & 1 & & 2 & 1 & 0 & 5 & 0 & & 1 & 1 & 2 & 0 & 0 & 4 \\
\hline CHSP & 0 & 0 & 0 & 0 & & 0 & 0 & 0 & 0 & 0 & 0 & 0 & 0 & 0 & 0 & 0 & 0 & 0 & 0 & 0 & & 0 & 0 & 0 & 0 & 0 & 0 & & 0 & 0 & 0 & 0 & 0 & & 0 & 0 & 0 & 0 & 0 & 0 \\
\hline SAGS & 0 & 0 & 0 & 0 & & 0 & 0 & 0 & 0 & 0 & 0 & 0 & 0 & 0 & 0 & 0 & 0 & 0 & 0 & 0 & & 0 & 0 & 0 & 0 & 0 & 0 & & 0 & 0 & 0 & 0 & 0 & & 0 & 0 & 0 & 0 & 0 & 0 \\
\hline SOSP & 0 & 0 & 0 & 0 & & 1 & 0 & 1 & 0 & 0 & 0 & 0 & 0 & 0 & 0 & 0 & 0 & 0 & 0 & 0 & & 0 & 0 & 0 & 0 & 0 & 0 & & 0 & 0 & 0 & 0 & 0 & & 0 & 0 & 0 & 0 & 0 & 0 \\
\hline WTSP & 0 & 0 & 0 & 0 & & 0 & 0 & 0 & 0 & 0 & 0 & 0 & 0 & 0 & 0 & 0 & 0 & 0 & 0 & 0 & & 0 & 0 & 0 & 0 & 0 & 0 & & 0 & 0 & 0 & 0 & 0 & & 0 & 0 & 0 & 0 & 0 & 0 \\
\hline ORJU & 0 & 0 & 0 & 0 & & 0 & 0 & 0 & 0 & 0 & 0 & 0 & 0 & 0 & 0 & 0 & 0 & 0 & 0 & 0 & & 0 & 0 & 0 & 0 & 0 & 0 & & 0 & 0 & 0 & 0 & 0 & & 0 & 0 & 0 & 0 & 0 & 0 \\
\hline BHGR & 0 & 0 & 0 & 0 & & 0 & 0 & 0 & 0 & 0 & 0 & 0 & 0 & 0 & 0 & 0 & 0 & 0 & 0 & 0 & & 0 & 0 & 0 & 0 & 0 & 0 & & 0 & 0 & 0 & 0 & 0 & & 0 & 0 & 0 & 0 & 0 & 0 \\
\hline$B L G R$ & 0 & 0 & 0 & 0 & & 0 & 0 & 0 & 0 & 0 & 0 & 0 & 0 & 0 & 0 & 0 & 0 & 0 & 0 & 0 & & 0 & 0 & 0 & 0 & 0 & 0 & & 0 & 0 & 0 & 0 & 0 & & 0 & 0 & 0 & 0 & 0 & 0 \\
\hline LAZB & 0 & 0 & 0 & 0 & & 0 & 0 & 0 & 0 & 0 & 0 & 0 & 0 & 0 & 0 & 0 & 0 & 0 & 0 & 0 & & 0 & 0 & 0 & 0 & 0 & 0 & & 0 & 0 & 0 & 0 & 0 & & 0 & 0 & 0 & 0 & 0 & 0 \\
\hline INBU & 0 & 0 & 0 & 0 & & 0 & 0 & 0 & 0 & 0 & 0 & 0 & 0 & 0 & 0 & 0 & 0 & 0 & 0 & 0 & & 0 & 0 & 0 & 0 & 0 & 0 & & 0 & 0 & 0 & 0 & 0 & c & 0 & 0 & 0 & 0 & 0 & 0 \\
\hline OROR & 0 & 0 & 0 & 0 & & 0 & 0 & 0 & 0 & 0 & 0 & 0 & 0 & 0 & 0 & 0 & 0 & 0 & 0 & 0 & & 0 & 0 & 0 & 0 & 0 & 0 & & 0 & 0 & 0 & 0 & 0 & & 0 & 0 & 0 & 0 & 0 & 0 \\
\hline HOOR & 0 & 0 & 0 & 0 & & 0 & 0 & 0 & 0 & 0 & 0 & 0 & 0 & 0 & 0 & 0 & 0 & 0 & 0 & 0 & & 0 & 0 & 0 & 0 & 0 & 0 & & 0 & 0 & 0 & 0 & 0 & & 0 & 0 & 0 & 1 & 0 & 1 \\
\hline BUOR & 0 & 0 & 0 & 0 & & 0 & 0 & 0 & 0 & 0 & 0 & 0 & 0 & 0 & 0 & 0 & 0 & 0 & 0 & 0 & & 0 & 0 & 0 & 0 & 0 & 0 & & 0 & 0 & 0 & 0 & 0 & c & 0 & 0 & 0 & 0 & 0 & 0 \\
\hline HOFI & 0 & 0 & 0 & 0 & & 0 & 0 & 0 & 0 & 0 & 0 & 0 & 0 & 0 & 0 & 0 & 0 & 0 & 0 & 0 & & 0 & 0 & 0 & 0 & 0 & 0 & & 0 & 0 & 0 & 0 & 0 & c & 0 & 0 & 0 & 0 & 0 & 0 \\
\hline LEGO & 0 & 0 & 0 & 0 & & 0 & 0 & 0 & 0 & 0 & 0 & 0 & 0 & 0 & 0 & 0 & 0 & 0 & 0 & 0 & & 0 & 0 & 0 & 0 & 0 & 0 & & 0 & 0 & 0 & 0 & 0 & c & 0 & 0 & 0 & 0 & 0 & 0 \\
\hline AMGO & 0 & 0 & 0 & 0 & & 0 & 0 & 0 & 0 & 0 & 0 & 0 & 0 & 0 & 0 & 0 & 0 & 0 & 0 & 0 & & 0 & 0 & 0 & 0 & 0 & 0 & & 0 & 0 & 0 & 0 & 0 & c & 0 & 0 & 0 & 0 & 0 & $\underline{0}$ \\
\hline Total & 1 & 3 & 3 & 3 & 2 & 0 & 11 & 41 & 1 & 1 & 4 & 6 & 18 & 8 & 38 & 2 & 1 & 1 & 1 & 6 & & 8 & 7 & 5 & 31 & 2 & 8 & & 6 & 8 & 4 & 28 & 5 & & 3 & 3 & 4 & 5 & 9 & 29 \\
\hline
\end{tabular}


Table C3. Capture rate by net and date during Monitoring Avian Productivity and Survivorship (MAPS), Point Loma banding station, San Diego, California, 2011.

\begin{tabular}{|c|c|c|c|c|c|c|c|c|c|c|c|c|c|}
\hline \multirow{2}{*}{$\begin{array}{l}\text { MAPS } \\
\text { period }\end{array}$} & \multirow[b]{2}{*}{ Date } & & \multicolumn{10}{|c|}{ Net } & \multirow{2}{*}{$\begin{array}{c}\text { Totals by } \\
\text { (hours:minutes) }\end{array}$} \\
\hline & & & 1 & 2 & 3 & 4 & 5 & 6 & 7 & 8 & 9 & 10 & \\
\hline \multirow[b]{3}{*}{1} & \multirow[b]{3}{*}{$5-05-11$} & \multirow{3}{*}{$\begin{array}{l}\text { Net hours (hours:minutes) } \\
\text { Captures (number) } \\
\text { Captures per net-hour }\end{array}$} & $5: 30$ & $4: 50$ & $4: 50$ & $5: 10$ & $5: 10$ & $5: 00$ & $4: 10$ & $5: 20$ & $5: 40$ & $5: 40$ & $51: 20$ \\
\hline & & & 13 & 5 & 5 & 9 & 2 & 3 & 5 & 8 & 10 & 7 & 67 \\
\hline & & & 2.36 & 1.03 & 1.03 & 1.74 & 0.39 & 0.60 & 0.00 & 1.50 & 1.76 & 0.00 & 1.31 \\
\hline \multirow[b]{3}{*}{2} & \multirow[b]{3}{*}{$5-12-11$} & \multirow{3}{*}{$\begin{array}{l}\text { Net hours (hours:minutes) } \\
\text { Captures (number) } \\
\text { Captures per net-hour }\end{array}$} & $5: 10$ & $5: 20$ & $5: 10$ & 5:00 & $4: 40$ & $4: 50$ & $2: 50$ & $4: 20$ & 5:00 & $4: 30$ & $46: 50$ \\
\hline & & & 14 & 15 & 11 & 16 & 11 & 7 & 12 & 14 & 9 & 10 & 119 \\
\hline & & & 2.71 & 2.81 & 2.13 & 3.20 & 2.36 & 1.45 & 4.24 & 3.23 & 1.80 & 0.00 & 2.54 \\
\hline \multirow[b]{3}{*}{3} & \multirow[b]{3}{*}{$5-26-11$} & \multirow{3}{*}{$\begin{array}{l}\text { Net hours (hours:minutes) } \\
\text { Captures (number) } \\
\text { Captures per net-hour }\end{array}$} & $5: 00$ & $5: 10$ & $4: 50$ & $5: 10$ & $4: 50$ & $4: 50$ & $4: 40$ & $4: 50$ & $5: 00$ & $5: 00$ & 49:20 \\
\hline & & & 12 & 6 & 5 & 12 & 8 & 2 & 4 & 4 & 10 & 6 & 69 \\
\hline & & & 2.40 & 1.16 & 1.03 & 2.32 & 1.66 & 0.41 & 0.86 & 0.83 & 2.00 & 1.20 & 1.40 \\
\hline \multirow[b]{3}{*}{4} & \multirow[b]{3}{*}{$6-02-11$} & \multirow{3}{*}{$\begin{array}{l}\text { Net hours (hours:minutes) } \\
\text { Captures (number) } \\
\text { Captures per net-hour }\end{array}$} & $5: 10$ & 5:00 & 5:00 & $5: 10$ & 5:00 & $4: 40$ & $4: 50$ & $4: 50$ & $5: 10$ & 5:00 & 49:50 \\
\hline & & & 10 & 2 & 2 & 7 & 7 & 2 & 4 & 11 & 3 & 2 & 50 \\
\hline & & & 1.94 & 0.40 & 0.40 & 1.35 & 1.40 & 0.43 & 0.83 & 2.28 & 0.58 & 0.40 & 1.00 \\
\hline \multirow[b]{3}{*}{5} & \multirow[b]{3}{*}{$6-16-11$} & \multirow{3}{*}{$\begin{array}{l}\text { Net hours (hours:minutes) } \\
\text { Captures (number) } \\
\text { Captures per net-hour }\end{array}$} & $5: 20$ & 5:00 & $4: 50$ & 5:00 & $4: 00$ & $5: 10$ & $5: 00$ & $5: 10$ & $4: 50$ & $4: 40$ & 49:00 \\
\hline & & & 10 & 2 & 3 & 1 & 0 & 1 & 0 & 1 & 2 & 8 & 28 \\
\hline & & & 1.88 & 0.40 & 0.62 & 0.00 & 0.00 & 0.19 & 0.00 & 0.19 & 0.00 & 1.71 & 0.57 \\
\hline \multirow[b]{3}{*}{6} & \multirow[b]{3}{*}{$6-23-11$} & \multirow{3}{*}{$\begin{array}{l}\text { Net hours (hours:minutes) } \\
\text { Captures (number) } \\
\text { Captures per net-hour }\end{array}$} & $5: 10$ & 5:00 & $5: 30$ & 5:00 & $5: 10$ & $4: 50$ & $5: 10$ & $5: 10$ & $5: 10$ & $5: 20$ & $51: 30$ \\
\hline & & & 3 & 2 & 3 & 0 & 0 & 8 & 1 & 5 & 1 & 9 & 32 \\
\hline & & & 0.58 & 0.40 & 0.55 & 0.00 & 0.00 & 1.66 & 0.19 & 0.97 & 0.19 & 1.69 & 0.62 \\
\hline \multirow[b]{3}{*}{7} & \multirow[b]{3}{*}{$7-06-11$} & \multirow{3}{*}{$\begin{array}{l}\text { Net hours (hours:minutes) } \\
\text { Captures (number) } \\
\text { Captures per net-hour }\end{array}$} & $5: 30$ & 5:00 & $4: 50$ & 5:00 & $4: 20$ & $4: 40$ & $4: 40$ & 5:00 & $5: 10$ & $5: 10$ & 49:20 \\
\hline & & & 8 & 3 & 2 & 6 & 1 & 2 & 8 & 2 & 4 & 11 & 47 \\
\hline & & & 1.45 & 0.60 & 0.41 & 1.20 & 0.23 & 0.43 & 1.71 & 0.40 & 0.77 & 2.13 & 0.95 \\
\hline \multirow[b]{3}{*}{8} & \multirow[b]{3}{*}{$7-14-11$} & \multirow{3}{*}{$\begin{array}{l}\text { Net hours (hours:minutes) } \\
\text { Captures (number) } \\
\text { Captures per net-hour }\end{array}$} & $5: 20$ & $4: 20$ & $4: 50$ & $4: 20$ & $4: 50$ & $4: 30$ & $5: 10$ & $5: 10$ & $4: 50$ & $4: 50$ & 48:10 \\
\hline & & & 2 & 1 & 3 & 3 & 1 & 0 & 0 & 1 & 13 & 7 & 31 \\
\hline & & & 0.38 & 0.23 & 0.62 & 0.00 & 0.21 & 0.00 & 0.00 & 0.19 & 2.69 & 1.45 & 0.64 \\
\hline & & Net hours (hours:minutes) & $4: 50$ & $4: 50$ & $4: 50$ & $4: 50$ & $4: 50$ & $5: 00$ & $5: 00$ & 5:00 & $5: 00$ & $5: 00$ & 49:10 \\
\hline & & Captures (number) & 4 & 0 & 0 & 2 & 0 & 2 & 0 & 8 & 6 & 2 & 24 \\
\hline 9 & $7-21-11$ & Captures per net-hour & 0.83 & 0.00 & 0.00 & 0.41 & 0.00 & 0.40 & 0.00 & 1.60 & 1.20 & 0.40 & 0.49 \\
\hline
\end{tabular}




\begin{tabular}{|c|c|c|c|c|c|c|c|c|c|c|c|c|c|}
\hline \multirow{2}{*}{$\begin{array}{l}\text { MAPS } \\
\text { period }\end{array}$} & \multirow[b]{2}{*}{ Date } & & \multicolumn{10}{|c|}{ Net } & \multirow{2}{*}{$\begin{array}{c}\text { Totals by } \\
\text { (hours:minutes) }\end{array}$} \\
\hline & & & 1 & 2 & 3 & 4 & 5 & 6 & 7 & 8 & 9 & 10 & \\
\hline \multirow[b]{3}{*}{10} & \multirow[b]{3}{*}{ 8-03-11 } & \multirow{3}{*}{$\begin{array}{l}\text { Net hours (hours:minutes) } \\
\text { Captures (number) } \\
\text { Captures per net-hour }\end{array}$} & $5: 10$ & $5: 10$ & $4: 50$ & $4: 40$ & $4: 50$ & $4: 20$ & $1: 40$ & $0: 40$ & $5: 20$ & $5: 10$ & $41: 50$ \\
\hline & & & 3 & 0 & 7 & 3 & 0 & 0 & 0 & 0 & 2 & 7 & 231 \\
\hline & & & 0.58 & 0.00 & 1.45 & 0.64 & 0.00 & 0.00 & 0.00 & 0.00 & 0.38 & 1.35 & 0.55 \\
\hline \multirow{3}{*}{\multicolumn{2}{|c|}{ Totals by net }} & \multirow{3}{*}{$\begin{array}{l}\text { Net hours (hours:minutes) } \\
\text { Captures (number) } \\
\text { Captures per net-hour }\end{array}$} & $52: 10$ & $49: 40$ & $49: 30$ & $49: 20$ & $47: 40$ & 47:50 & $43: 10$ & $45: 30$ & $51: 10$ & $50: 20$ & $486: 20$ \\
\hline & & & 79 & 36 & 41 & 59 & 30 & 27 & 34 & 54 & 60 & 69 & 4901 \\
\hline & & & 1.51 & 0.72 & 0.83 & 1.20 & 0.63 & 0.56 & 0.79 & 1.19 & 1.17 & 1.37 & 1.01 \\
\hline
\end{tabular}

${ }^{1}$ Total includes one bird captured with no net number recorded. 
Table C4. Capture rate by net and date during Monitoring Avian Productivity and Survivorship (MAPS), Point Loma banding station, San Diego, California, 2012.

\begin{tabular}{|c|c|c|c|c|c|c|c|c|c|c|c|c|c|}
\hline \multirow{2}{*}{$\begin{array}{l}\text { MAPS } \\
\text { period }\end{array}$} & \multirow[b]{2}{*}{ Date } & & \multicolumn{10}{|c|}{ Net } & \multirow{2}{*}{$\begin{array}{c}\text { Total } \\
\text { (hours:minutes) }\end{array}$} \\
\hline & & & 1 & 2 & 3 & 4 & 5 & 6 & 7 & 8 & 9 & 10 & \\
\hline \multirow[b]{3}{*}{1} & \multirow[b]{3}{*}{ 5-08-2012 } & \multirow{3}{*}{$\begin{array}{l}\text { Net-hours (hours:minutes) } \\
\text { Captures (number) } \\
\text { Captures per net-hour }\end{array}$} & $5: 10$ & $5: 10$ & $5: 10$ & $5: 10$ & $5: 10$ & $5: 10$ & $5: 10$ & $5: 10$ & $5: 10$ & $5: 10$ & $51: 40$ \\
\hline & & & 4 & 7 & 3 & 13 & 13 & 9 & 7 & 14 & 9 & 9 & 88 \\
\hline & & & 0.77 & 1.35 & 0.58 & 2.52 & 2.52 & 1.74 & 1.35 & 2.71 & 1.74 & 1.74 & 1.70 \\
\hline \multirow[b]{3}{*}{2} & \multirow[b]{3}{*}{$5-15-2012$} & \multirow{3}{*}{$\begin{array}{l}\text { Net-hours (hours:minutes) } \\
\text { Captures (number) } \\
\text { Captures per net-hour }\end{array}$} & 5:00 & $5: 10$ & $5: 10$ & $5: 10$ & $5: 10$ & $5: 00$ & $5: 20$ & $5: 10$ & 5:00 & 5:00 & $51: 10$ \\
\hline & & & 5 & 3 & 1 & 11 & 5 & 3 & 5 & 11 & 9 & 10 & 63 \\
\hline & & & 1.00 & 0.58 & 0.19 & 2.13 & 0.97 & 0.60 & 0.94 & 2.13 & 1.80 & 2.00 & 1.23 \\
\hline \multirow[b]{3}{*}{3} & \multirow[b]{3}{*}{$5-29-2012$} & \multirow{3}{*}{$\begin{array}{l}\text { Net-hours (hours:minutes) } \\
\text { Captures (number) } \\
\text { Captures per net-hour }\end{array}$} & 5:00 & 5:00 & $4: 50$ & $5: 00$ & $5: 00$ & $5: 00$ & $5: 00$ & 5:00 & $5: 10$ & $5: 00$ & $50: 00$ \\
\hline & & & 4 & 3 & 5 & 2 & 2 & 2 & 4 & 3 & 6 & 1 & 32 \\
\hline & & & 0.80 & 0.60 & 1.03 & 0.40 & 0.40 & 0.40 & 0.80 & 0.60 & 1.16 & 0.20 & 0.64 \\
\hline \multirow[b]{3}{*}{4} & \multirow[b]{3}{*}{$6-05-2012$} & \multirow{3}{*}{$\begin{array}{l}\text { Net-hours (hours:minutes) } \\
\text { Captures (number) } \\
\text { Captures per net-hour }\end{array}$} & $5: 10$ & $5: 10$ & $5: 10$ & $5: 00$ & $5: 20$ & $2: 40$ & $5: 10$ & $5: 10$ & $5: 10$ & $5: 00$ & $49: 00$ \\
\hline & & & 11 & 2 & 1 & 1 & 1 & 0 & 1 & 5 & 1 & 1 & 24 \\
\hline & & & 2.13 & 0.39 & 0.19 & 0.20 & 0.19 & 0.00 & 0.19 & 0.97 & 0.19 & 0.20 & 0.49 \\
\hline \multirow[b]{3}{*}{5} & \multirow[b]{3}{*}{$6-19-2012$} & \multirow{3}{*}{$\begin{array}{l}\text { Net-hours (hours:minutes) } \\
\text { Captures (number) } \\
\text { Captures per net-hour }\end{array}$} & 5:00 & $5: 20$ & $5: 10$ & $5: 10$ & $5: 10$ & $4: 40$ & $4: 40$ & $5: 10$ & $5: 00$ & $4: 50$ & $50: 10$ \\
\hline & & & 4 & 2 & 0 & 1 & 0 & 2 & 0 & 4 & 1 & 1 & 15 \\
\hline & & & 0.80 & 0.38 & 0.00 & 0.19 & 0.00 & 0.43 & 0.00 & 0.77 & 0.20 & 0.21 & 1.06 \\
\hline \multirow[b]{3}{*}{6} & \multirow[b]{3}{*}{$6-26-2012$} & \multirow{3}{*}{$\begin{array}{l}\text { Net-hours (hours:minutes) } \\
\text { Captures (number) } \\
\text { Captures per net-hour }\end{array}$} & $5: 00$ & $5: 10$ & 5:00 & 5:00 & $5: 10$ & $5: 10$ & $4: 20$ & $5: 10$ & $5: 00$ & $5: 00$ & $50: 00$ \\
\hline & & & 3 & 2 & 5 & 3 & 1 & 0 & 1 & 2 & 1 & 6 & 24 \\
\hline & & & 0.60 & 0.39 & 1.00 & 0.60 & 0.19 & 0.00 & 0.23 & 0.39 & 0.20 & 1.20 & 0.48 \\
\hline \multirow[b]{3}{*}{7} & \multirow[b]{3}{*}{$7-03-2012$} & \multirow{3}{*}{$\begin{array}{l}\text { Net-hours (hours:minutes) } \\
\text { Captures (number) } \\
\text { Captures per net-hour }\end{array}$} & 5:00 & $5: 10$ & $5: 10$ & 5:00 & $5: 10$ & $5: 10$ & $4: 50$ & 5:00 & $5: 10$ & 5:00 & $50: 40$ \\
\hline & & & 1 & 1 & 2 & 0 & 0 & 0 & 0 & 3 & 0 & 2 & 9 \\
\hline & & & 0.20 & 0.19 & 0.39 & 0.00 & 0.00 & 0.00 & 0.00 & 0.60 & 0.00 & 0.40 & 0.18 \\
\hline \multirow[b]{3}{*}{8} & \multirow[b]{3}{*}{$7-10-2012$} & \multirow{3}{*}{$\begin{array}{l}\text { Net-hours (hours:minutes) } \\
\text { Captures (number) } \\
\text { Captures per net-hour }\end{array}$} & $5: 00$ & 5:00 & $5: 10$ & $4: 00$ & $5: 10$ & $5: 00$ & $3: 20$ & $5: 00$ & $5: 00$ & $5: 10$ & $47: 50$ \\
\hline & & & 3 & 5 & 2 & 0 & 3 & 0 & 0 & 8 & 2 & 3 & 26 \\
\hline & & & 0.60 & 1.00 & 0.39 & 0.00 & 0.58 & 0.00 & 0.00 & 1.60 & 0.40 & 0.58 & 0.54 \\
\hline & & Net-hours (hours:minutes) & 5:00 & 5:00 & 5:00 & 5:00 & $5: 10$ & $4: 20$ & $5: 10$ & $5: 20$ & $5: 00$ & $5: 00$ & $50: 00$ \\
\hline & & Captures (number) & 3 & 1 & 4 & 5 & 0 & 1 & 0 & 3 & 15 & 2 & 34 \\
\hline 9 & $7-24-2012$ & Captures per net-hour & 0.60 & 0.20 & 0.80 & 1.00 & 0.00 & 0.23 & 0.00 & 0.56 & 3.00 & 0.40 & 0.66 \\
\hline
\end{tabular}




\begin{tabular}{|c|c|c|c|c|c|c|c|c|c|c|c|c|c|}
\hline \multirow{2}{*}{$\begin{array}{l}\text { MAPS } \\
\text { period }\end{array}$} & \multirow[b]{2}{*}{ Date } & & \multicolumn{10}{|c|}{ Net } & \multirow{2}{*}{$\begin{array}{c}\text { Total } \\
\text { (hours:minutes) }\end{array}$} \\
\hline & & & 1 & 2 & 3 & 4 & 5 & 6 & 7 & 8 & 9 & 10 & \\
\hline \multirow[b]{3}{*}{10} & \multirow[b]{3}{*}{ 8-07-2012 } & \multirow{3}{*}{$\begin{array}{l}\text { Net-hours (hours:minutes) } \\
\text { Captures (number) } \\
\text { Captures per net-hour }\end{array}$} & $5: 00$ & 5:00 & 5:00 & 5:00 & $4: 50$ & $5: 10$ & $5: 00$ & $5: 20$ & $5: 10$ & 5:00 & $50: 30$ \\
\hline & & & 2 & 0 & 0 & 7 & 0 & 2 & 0 & 6 & 0 & 2 & 19 \\
\hline & & & 0.40 & 0.00 & 0.00 & 1.40 & 0.00 & 0.39 & 0.00 & 1.13 & 0.00 & 0.40 & 0.38 \\
\hline \multirow{3}{*}{\multicolumn{2}{|c|}{ Totals by net }} & \multirow{3}{*}{$\begin{array}{l}\text { Net-hours (hours:minutes) } \\
\text { Captures (number) } \\
\text { Captures per net-hour }\end{array}$} & $50: 20$ & $51: 10$ & $50: 50$ & 49:30 & $51: 20$ & $47: 20$ & 48:00 & $51: 30$ & $50: 50$ & $50: 10$ & $501: 00$ \\
\hline & & & 40 & 26 & 23 & 43 & 25 & 19 & 18 & 59 & 44 & 37 & 334 \\
\hline & & & 0.79 & 0.51 & 0.45 & 0.87 & 0.49 & 0.40 & 0.38 & 1.15 & 0.87 & 0.74 & 0.67 \\
\hline
\end{tabular}


Table C5. Capture rate by net and date during Monitoring Avian Productivity and Survivorship (MAPS), Point Loma banding station, San Diego, California, 2013.

\begin{tabular}{|c|c|c|c|c|c|c|c|c|c|c|c|c|c|}
\hline \multirow{2}{*}{$\begin{array}{l}\text { MAPS } \\
\text { period }\end{array}$} & \multirow[b]{2}{*}{ Date } & & \multicolumn{10}{|c|}{ Net } & \multirow{2}{*}{$\begin{array}{c}\text { Total } \\
\text { (hours:minutes) }\end{array}$} \\
\hline & & & 1 & 2 & 3 & 4 & 5 & 6 & 7 & 8 & 9 & 10 & \\
\hline \multirow[b]{3}{*}{1} & \multirow[b]{3}{*}{$5-07-2013$} & \multirow{3}{*}{$\begin{array}{l}\text { Net-hours (hours:minutes) } \\
\text { Captures (number) } \\
\text { Captures per net-hour }\end{array}$} & $5: 10$ & 5:00 & $5: 20$ & $5: 00$ & 5:00 & $5: 10$ & $5: 00$ & $5: 10$ & $5: 00$ & $0: 00$ & $45: 50$ \\
\hline & & & 4 & 3 & 5 & 2 & 2 & 2 & 0 & 3 & 13 & 0 & 34 \\
\hline & & & 0.77 & 0.60 & 0.94 & 0.40 & 0.40 & 0.39 & 0.00 & 0.58 & 2.60 & 0.00 & 0.74 \\
\hline \multirow[b]{3}{*}{2} & \multirow[b]{3}{*}{ 5-14-2013 } & \multirow{3}{*}{$\begin{array}{l}\text { Net-hours (hours:minutes) } \\
\text { Captures (number) } \\
\text { Captures per net-hour }\end{array}$} & 5:00 & $5: 10$ & $5: 10$ & $5: 10$ & $5: 20$ & $5: 20$ & $4: 40$ & $5: 10$ & 5:00 & $5: 00$ & $51: 00$ \\
\hline & & & 8 & 4 & 11 & 6 & 5 & 1 & 0 & 6 & 3 & 8 & 52 \\
\hline & & & 1.60 & 0.77 & 2.13 & 1.16 & 0.94 & 0.19 & 0.00 & 1.16 & 0.60 & 1.60 & 1.02 \\
\hline \multirow[b]{3}{*}{3} & \multirow[b]{3}{*}{$5-28-2013$} & \multirow{3}{*}{$\begin{array}{l}\text { Net-hours (hours:minutes) } \\
\text { Captures (number) } \\
\text { Captures per net-hour }\end{array}$} & 5:00 & 5:00 & 5:00 & 5:00 & $5: 00$ & $5: 00$ & $5: 00$ & $5: 00$ & 5:00 & $5: 00$ & $50: 00$ \\
\hline & & & 9 & 3 & 2 & 0 & 1 & 1 & 1 & 2 & 5 & 7 & 31 \\
\hline & & & 1.80 & 0.60 & 0.40 & 0.00 & 0.20 & 0.20 & 0.20 & 0.40 & 1.00 & 1.40 & 0.62 \\
\hline \multirow[b]{3}{*}{4} & \multirow[b]{3}{*}{$6-04-2013$} & \multirow{3}{*}{$\begin{array}{l}\text { Net-hours (hours:minutes) } \\
\text { Captures (number) } \\
\text { Captures per net-hour }\end{array}$} & $5: 10$ & 5:00 & 5:00 & 5:00 & 5:00 & $5: 10$ & $4: 10$ & $4: 50$ & 5:00 & 5:00 & 49:20 \\
\hline & & & 2 & 3 & 5 & 2 & 4 & 4 & 2 & 3 & 2 & 3 & 30 \\
\hline & & & 0.39 & 0.60 & 1.00 & 0.40 & 0.80 & 0.77 & 0.48 & 0.62 & 0.40 & 0.60 & 0.61 \\
\hline \multirow[b]{3}{*}{5} & \multirow[b]{3}{*}{ 6-18-2013 } & \multirow{3}{*}{$\begin{array}{l}\text { Net-hours (hours:minutes) } \\
\text { Captures (number) } \\
\text { Captures per net-hour }\end{array}$} & $4: 40$ & 5:00 & 5:00 & $4: 50$ & $5: 10$ & $5: 10$ & $5: 00$ & $5: 10$ & $5: 00$ & $5: 00$ & 50:00 \\
\hline & & & 3 & 13 & 1 & 0 & 3 & 0 & 0 & 0 & 2 & 1 & 23 \\
\hline & & & 0.64 & 2.60 & 0.20 & 0.00 & 0.58 & 0.00 & 0.00 & 0.00 & 0.40 & 0.20 & 1.06 \\
\hline \multirow[b]{3}{*}{6} & \multirow[b]{3}{*}{$6-25-2013$} & \multirow{3}{*}{$\begin{array}{l}\text { Net-hours (hours:minutes) } \\
\text { Captures (number) } \\
\text { Captures per net-hour }\end{array}$} & 5:00 & 5:00 & 5:00 & 5:00 & 5:00 & 5:00 & 5:00 & 5:00 & 5:00 & 5:00 & $50: 00$ \\
\hline & & & 2 & 2 & 5 & 3 & 0 & 0 & 2 & 3 & 2 & 1 & 20 \\
\hline & & & 0.40 & 0.40 & 1.00 & 0.60 & 0.00 & 0.00 & 0.40 & 0.60 & 0.40 & 0.20 & 0.40 \\
\hline \multirow[b]{3}{*}{7} & \multirow[b]{3}{*}{$7-02-2013$} & \multirow{3}{*}{$\begin{array}{l}\text { Net-hours (hours:minutes) } \\
\text { Captures (number) } \\
\text { Captures per net-hour }\end{array}$} & 5:00 & $5: 10$ & 5:00 & 5:00 & $5: 10$ & $5: 20$ & 5:00 & $5: 10$ & $5: 10$ & 5:00 & $51: 00$ \\
\hline & & & 2 & 2 & 1 & 2 & 2 & 1 & 1 & 2 & 0 & 1 & 14 \\
\hline & & & 0.40 & 0.39 & 0.20 & 0.40 & 0.39 & 0.19 & 0.20 & 0.39 & 0.00 & 0.20 & 0.27 \\
\hline \multirow[b]{3}{*}{8} & \multirow[b]{3}{*}{$7-16-2013$} & \multirow{3}{*}{$\begin{array}{l}\text { Net-hours (hours:minutes) } \\
\text { Captures (number) } \\
\text { Captures per net-hour }\end{array}$} & 5:00 & 5:00 & $5: 00$ & $5: 10$ & $5: 20$ & $5: 10$ & 5:00 & $5: 10$ & 5:00 & $5: 10$ & $51: 00$ \\
\hline & & & 3 & 2 & 4 & 4 & 2 & 3 & 0 & 6 & 3 & 2 & 29 \\
\hline & & & 0.60 & 0.40 & 0.80 & 0.77 & 0.38 & 0.58 & 0.00 & 1.16 & 0.60 & 0.39 & 0.57 \\
\hline & & Net-hours (hours:minutes) & 5:00 & $5: 00$ & 5:00 & $5: 20$ & $5: 00$ & $5: 00$ & $5: 00$ & $5: 10$ & $5: 00$ & $5: 00$ & $50: 30$ \\
\hline & & Captures (number) & 1 & 2 & 2 & 2 & 0 & 0 & 1 & 12 & 0 & 1 & 21 \\
\hline 9 & $7-23-2013$ & Captures per net-hour & 0.20 & 0.40 & 0.40 & 0.38 & 0.00 & 0.00 & 0.20 & 2.32 & 0.00 & 0.20 & 0.65 \\
\hline
\end{tabular}




\begin{tabular}{|c|c|c|c|c|c|c|c|c|c|c|c|c|c|}
\hline \multirow{2}{*}{$\begin{array}{l}\text { MAPS } \\
\text { period }\end{array}$} & \multirow[b]{2}{*}{ Date } & & \multicolumn{10}{|c|}{ Net } & \multirow{2}{*}{$\begin{array}{c}\text { Total } \\
\text { (hours:minutes) }\end{array}$} \\
\hline & & & 1 & 2 & 3 & 4 & 5 & 6 & 7 & 8 & 9 & 10 & \\
\hline \multirow[b]{3}{*}{10} & \multirow[b]{3}{*}{$8-06-2013$} & \multirow{3}{*}{$\begin{array}{l}\text { Net-hours (hours:minutes) } \\
\text { Captures (number) } \\
\text { Captures per net-hour }\end{array}$} & $5: 10$ & $5: 00$ & $5: 10$ & 4:00 & $5: 20$ & $5: 10$ & $4: 40$ & $5: 10$ & $5: 20$ & $5: 20$ & $50: 20$ \\
\hline & & & 4 & 2 & 0 & 1 & 0 & 0 & 2 & 0 & 3 & 1 & 13 \\
\hline & & & 0.77 & 0.40 & 0.00 & 0.25 & 0.00 & 0.00 & 0.43 & 0.00 & 0.56 & 0.19 & 0.26 \\
\hline \multirow{3}{*}{\multicolumn{2}{|c|}{ Totals by net }} & \multirow{3}{*}{$\begin{array}{l}\text { Net-hours (hours:minutes) } \\
\text { Captures (number) } \\
\text { Captures per net-hour }\end{array}$} & $50: 10$ & $50: 20$ & $50: 40$ & $49: 30$ & $51: 20$ & $51: 30$ & $48: 30$ & $51: 00$ & $50: 30$ & $45: 30$ & 499:00 \\
\hline & & & 38 & 36 & 36 & 22 & 19 & 12 & 9 & 37 & 33 & 25 & 267 \\
\hline & & & 0.76 & 0.72 & 0.71 & 0.44 & 0.37 & 0.23 & 0.19 & 0.73 & 0.65 & 0.55 & 0.54 \\
\hline
\end{tabular}


Table C6. Capture rate by net and date during Monitoring Avian Productivity and Survivorship (MAPS), Point Loma banding station, San Diego, California, 2014.

\begin{tabular}{|c|c|c|c|c|c|c|c|c|c|c|c|c|c|}
\hline \multirow{2}{*}{$\begin{array}{l}\text { MAPS } \\
\text { period }\end{array}$} & \multirow[b]{2}{*}{ Date } & & \multicolumn{10}{|c|}{ Net } & \multirow{2}{*}{$\begin{array}{c}\text { Total } \\
\text { (hours:minutes) }\end{array}$} \\
\hline & & & 1 & 2 & 3 & 4 & 5 & 6 & 7 & 8 & 9 & 10 & \\
\hline \multirow[b]{3}{*}{1} & \multirow[b]{3}{*}{$5-9-2014$} & \multirow{3}{*}{$\begin{array}{l}\text { Net-hours (hours:minutes) } \\
\text { Captures (number) } \\
\text { Captures per net-hour }\end{array}$} & $5: 10$ & $5: 20$ & $5: 10$ & $5: 10$ & $5: 10$ & $5: 10$ & $5: 10$ & $5: 10$ & $5: 10$ & $5: 00$ & $51: 40$ \\
\hline & & & 13 & 5 & 10 & 9 & 6 & 9 & 4 & 14 & 6 & 16 & 92 \\
\hline & & & 2.52 & 0.94 & 1.94 & 1.74 & 1.16 & 1.74 & 0.77 & 2.71 & 1.16 & 0.00 & 1.78 \\
\hline \multirow[b]{3}{*}{2} & \multirow[b]{3}{*}{$5-15-2014$} & \multirow{3}{*}{$\begin{array}{l}\text { Net-hours (hours:minutes) } \\
\text { Captures (number) } \\
\text { Captures per net-hour }\end{array}$} & $4: 40$ & $4: 20$ & $4: 30$ & $4: 20$ & $4: 10$ & $4: 30$ & $4: 20$ & $4: 20$ & $4: 20$ & $4: 20$ & $43: 50$ \\
\hline & & & 8 & 4 & 16 & 5 & 9 & 6 & 2 & 10 & 8 & 7 & 75 \\
\hline & & & 1.71 & 0.92 & 3.56 & 1.15 & 2.16 & 1.33 & 0.46 & 2.31 & 1.85 & 1.62 & 1.71 \\
\hline \multirow[b]{3}{*}{3} & \multirow[b]{3}{*}{$5-27-2014$} & \multirow{3}{*}{$\begin{array}{l}\text { Net-hours (hours:minutes) } \\
\text { Captures (number) } \\
\text { Captures per net-hour }\end{array}$} & $5: 10$ & $5: 20$ & $5: 10$ & $5: 10$ & $5: 10$ & $5: 20$ & $5: 00$ & $5: 10$ & $5: 00$ & $5: 10$ & $51: 40$ \\
\hline & & & 9 & 9 & 13 & 20 & 6 & 6 & 5 & 6 & 7 & 12 & 93 \\
\hline & & & 1.74 & 1.69 & 2.52 & 3.87 & 1.16 & 1.13 & 1.00 & 1.16 & 1.40 & 2.32 & 1.80 \\
\hline \multirow[b]{3}{*}{4} & \multirow[b]{3}{*}{ 6-3-2014 } & \multirow{3}{*}{$\begin{array}{l}\text { Net-hours (hours:minutes) } \\
\text { Captures (number) } \\
\text { Captures per net-hour }\end{array}$} & 5:00 & 5:00 & 5:00 & 5:00 & 5:00 & $5: 00$ & 5:00 & $5: 00$ & 5:00 & 5:00 & $50: 00$ \\
\hline & & & 1 & 1 & 3 & 1 & 0 & 1 & 3 & 1 & 3 & 1 & 15 \\
\hline & & & 0.20 & 0.20 & 0.60 & 0.20 & 0.00 & 0.20 & 0.60 & 0.20 & 0.60 & 0.20 & 0.30 \\
\hline \multirow[b]{3}{*}{5} & \multirow[b]{3}{*}{$6-17-2014$} & \multirow{3}{*}{$\begin{array}{l}\text { Net-hours (hours:minutes) } \\
\text { Captures (number) } \\
\text { Captures per net-hour }\end{array}$} & $5: 00$ & 5:00 & $5: 10$ & $4: 00$ & 5:00 & $4: 00$ & $4: 10$ & $5: 00$ & $5: 00$ & $5: 00$ & $47: 20$ \\
\hline & & & 0 & 0 & 1 & 4 & 0 & 1 & 1 & 2 & 6 & 4 & 19 \\
\hline & & & 0.00 & 0.00 & 0.19 & 1.00 & 0.00 & 0.25 & 0.24 & 0.40 & 1.20 & 0.80 & 1.12 \\
\hline \multirow[b]{3}{*}{6} & \multirow[b]{3}{*}{$6-24-2014$} & \multirow{3}{*}{$\begin{array}{l}\text { Net-hours (hours:minutes) } \\
\text { Captures (number) } \\
\text { Captures per net-hour }\end{array}$} & 5:00 & $5: 10$ & $5: 10$ & $5: 00$ & $5: 10$ & $5: 10$ & 5:00 & $5: 10$ & $5: 10$ & $5: 10$ & $51: 10$ \\
\hline & & & 0 & 0 & 2 & 0 & 1 & 0 & 0 & 2 & 3 & 2 & 10 \\
\hline & & & 0.00 & 0.00 & 0.39 & 0.00 & 0.19 & 0.00 & 0.00 & 0.39 & 0.58 & 0.39 & 0.20 \\
\hline \multirow[b]{3}{*}{7} & \multirow[b]{3}{*}{$7-8-2014$} & \multirow{3}{*}{$\begin{array}{l}\text { Net-hours (hours:minutes) } \\
\text { Captures (number) } \\
\text { Captures per net-hour }\end{array}$} & $5: 10$ & $5: 10$ & $5: 10$ & $5: 20$ & $5: 10$ & $5: 10$ & $5: 10$ & $5: 10$ & $5: 10$ & 5:00 & $51: 40$ \\
\hline & & & 2 & 0 & 1 & 4 & 0 & 0 & 0 & 8 & 0 & 2 & 17 \\
\hline & & & 0.39 & 0.00 & 0.19 & 0.75 & 0.00 & 0.00 & 0.00 & 1.55 & 0.00 & 0.40 & 0.33 \\
\hline \multirow[b]{3}{*}{8} & \multirow[b]{3}{*}{$7-15-2014$} & \multirow{3}{*}{$\begin{array}{l}\text { Net-hours (hours:minutes) } \\
\text { Captures (number) } \\
\text { Captures per net-hour }\end{array}$} & 5:00 & $5: 10$ & $5: 10$ & $5: 10$ & $5: 00$ & $5: 10$ & $5: 10$ & $5: 10$ & $5: 00$ & 5:00 & 51:00 \\
\hline & & & 1 & 0 & 0 & 1 & 0 & 0 & 1 & 1 & 2 & 0 & 6 \\
\hline & & & 0.20 & 0.00 & 0.00 & 0.19 & 0.00 & 0.00 & 0.19 & 0.19 & 0.40 & 0.00 & 0.12 \\
\hline & & Net-hours (hours:minutes) & $5: 10$ & 5:00 & $5: 10$ & 5:00 & $5: 10$ & $5: 00$ & $4: 30$ & $5: 00$ & $5: 10$ & $5: 00$ & $50: 10$ \\
\hline & & Captures (number) & 0 & 1 & 0 & 1 & 1 & 2 & 0 & 0 & 3 & 0 & 8 \\
\hline 9 & $7-22-2014$ & Captures per net-hour & 0.00 & 0.20 & 0.00 & 0.20 & 0.19 & 0.40 & 0.00 & 0.00 & 0.58 & 0.00 & 0.66 \\
\hline
\end{tabular}




\begin{tabular}{|c|c|c|c|c|c|c|c|c|c|c|c|c|c|}
\hline \multirow{2}{*}{$\begin{array}{l}\text { MAPS } \\
\text { period }\end{array}$} & \multirow[b]{2}{*}{ Date } & & \multicolumn{10}{|c|}{ Net } & \multirow{2}{*}{$\begin{array}{c}\text { Total } \\
\text { (hours:minutes) }\end{array}$} \\
\hline & & & 1 & 2 & 3 & 4 & 5 & 6 & 7 & 8 & 9 & 10 & \\
\hline \multirow[b]{3}{*}{10} & \multirow[b]{3}{*}{$8-5-2014$} & \multirow{3}{*}{$\begin{array}{l}\text { Net-hours (hours:minutes) } \\
\text { Captures (number) } \\
\text { Captures per net-hour }\end{array}$} & $5: 00$ & $5: 10$ & 4:50 & $4: 40$ & $5: 00$ & $5: 00$ & $5: 00$ & $4: 50$ & $4: 50$ & $4: 50$ & $49: 10$ \\
\hline & & & 2 & 1 & 0 & 0 & 0 & 0 & 0 & 1 & 1 & 1 & 6 \\
\hline & & & 0.40 & 0.19 & 0.00 & 0.00 & 0.00 & 0.00 & 0.00 & 0.21 & 0.21 & 0.21 & 0.12 \\
\hline \multirow{3}{*}{\multicolumn{2}{|c|}{ Totals by net }} & \multirow{3}{*}{$\begin{array}{l}\text { Net-hours (hours:minutes) } \\
\text { Captures (number) } \\
\text { Captures per net-hour }\end{array}$} & $50: 20$ & $50: 40$ & $50: 30$ & 48:50 & $50: 00$ & $49: 30$ & $48: 30$ & $50: 00$ & $49: 50$ & $49: 30$ & $497: 40$ \\
\hline & & & 36 & 21 & 46 & 45 & 23 & 25 & 16 & 45 & 39 & 45 & 341 \\
\hline & & & 0.72 & 0.41 & 0.91 & 0.92 & 0.46 & 0.51 & 0.33 & 0.90 & 0.78 & 0.91 & 0.69 \\
\hline
\end{tabular}


Table C7. Capture rate by net and date during Monitoring Avian Productivity and Survivorship (MAPS), Point Loma banding station, San Diego, California, 2015.

\begin{tabular}{|c|c|c|c|c|c|c|c|c|c|c|c|c|c|}
\hline \multirow{2}{*}{$\begin{array}{l}\text { MAPS } \\
\text { period }\end{array}$} & \multirow[b]{2}{*}{ Date } & & \multicolumn{10}{|c|}{ Net } & \multirow{2}{*}{$\begin{array}{c}\text { Total } \\
\text { (hours:minutes) }\end{array}$} \\
\hline & & & 1 & 2 & 3 & 4 & 5 & 6 & 7 & 8 & 9 & 10 & \\
\hline \multirow[b]{3}{*}{1} & \multirow[b]{3}{*}{$5-5-2015$} & \multirow{3}{*}{$\begin{array}{l}\text { Net-hours (hours:minutes) } \\
\text { Captures (number) } \\
\text { Captures per net-hour }\end{array}$} & 5:00 & 5:00 & 5:00 & 5:00 & 5:00 & 5:00 & $4: 10$ & 5:00 & $5: 00$ & $5: 00$ & $49: 10$ \\
\hline & & & 8 & 11 & 6 & 4 & 3 & 8 & 1 & 11 & 11 & 8 & 71 \\
\hline & & & 1.60 & 2.20 & 1.20 & 0.80 & 0.60 & 1.60 & 0.24 & 2.20 & 2.20 & 0.00 & 1.44 \\
\hline \multirow[b]{3}{*}{2} & \multirow[b]{3}{*}{$5-12-2015$} & \multirow{3}{*}{$\begin{array}{l}\text { Net-hours (hours:minutes) } \\
\text { Captures (number) } \\
\text { Captures per net-hour }\end{array}$} & $5: 30$ & $5: 10$ & $5: 10$ & 5:00 & $3: 00$ & 5:00 & $5: 10$ & $5: 10$ & $5: 10$ & $5: 10$ & 49:30 \\
\hline & & & 14 & 5 & 3 & 11 & 2 & 3 & 10 & 8 & 5 & 8 & 69 \\
\hline & & & 2.55 & 0.97 & 0.58 & 2.20 & 0.67 & 0.60 & 1.94 & 1.55 & 0.97 & 1.55 & 1.39 \\
\hline \multirow[b]{3}{*}{3} & \multirow[b]{3}{*}{ 5-26-2015 } & \multirow{3}{*}{$\begin{array}{l}\text { Net-hours (hours:minutes) } \\
\text { Captures (number) } \\
\text { Captures per net-hour }\end{array}$} & $5: 10$ & 5:00 & 5:00 & 5:00 & $4: 50$ & 5:00 & $5: 10$ & $4: 50$ & $5: 00$ & $5: 10$ & $50: 10$ \\
\hline & & & 7 & 3 & 1 & 5 & 6 & 5 & 0 & 5 & 7 & 10 & $50^{1}$ \\
\hline & & & 1.35 & 0.60 & 0.20 & 1.00 & 1.24 & 1.00 & 0.00 & 1.03 & 1.40 & 1.94 & 1.00 \\
\hline \multirow[b]{3}{*}{4} & \multirow[b]{3}{*}{$6-2-2015$} & \multirow{3}{*}{$\begin{array}{l}\text { Net-hours (hours:minutes) } \\
\text { Captures (number) } \\
\text { Captures per net-hour }\end{array}$} & $4: 50$ & 5:00 & 5:00 & $4: 50$ & $4: 40$ & $4: 50$ & $4: 50$ & 5:00 & 5:00 & $5: 20$ & 49:20 \\
\hline & & & 4 & 1 & 1 & 1 & 1 & 0 & 1 & 1 & 0 & 7 & 17 \\
\hline & & & 0.83 & 0.20 & 0.20 & 0.21 & 0.21 & 0.00 & 0.21 & 0.20 & 0.00 & 1.31 & 0.34 \\
\hline \multirow[b]{3}{*}{5} & \multirow[b]{3}{*}{ 6-16-2015 } & \multirow{3}{*}{$\begin{array}{l}\text { Net-hours (hours:minutes) } \\
\text { Captures (number) } \\
\text { Captures per net-hour }\end{array}$} & 5:00 & 5:00 & 5:00 & $5: 10$ & $5: 10$ & 5:00 & 5:00 & 5:00 & 5:00 & 5:00 & $50: 20$ \\
\hline & & & 1 & 2 & 1 & 3 & 1 & 0 & 1 & 0 & 2 & 3 & 14 \\
\hline & & & 0.20 & 0.40 & 0.20 & 0.58 & 0.19 & 0.00 & 0.20 & 0.00 & 0.40 & 0.60 & 1.05 \\
\hline \multirow[b]{3}{*}{6} & \multirow[b]{3}{*}{ 6-23-2015 } & \multirow{3}{*}{$\begin{array}{l}\text { Net-hours (hours:minutes) } \\
\text { Captures (number) } \\
\text { Captures per net-hour }\end{array}$} & 5:00 & 5:00 & 5:00 & $4: 50$ & $4: 50$ & 5:00 & $5: 20$ & 5:00 & 5:00 & $5: 10$ & $50: 10$ \\
\hline & & & 1 & 4 & 3 & 1 & 0 & 1 & 1 & 3 & 5 & 3 & 22 \\
\hline & & & 0.20 & 0.80 & 0.60 & 0.21 & 0.00 & 0.20 & 0.19 & 0.60 & 1.00 & 0.58 & 0.44 \\
\hline \multirow[b]{3}{*}{7} & \multirow[b]{3}{*}{$7-7-2015$} & \multirow{3}{*}{$\begin{array}{l}\text { Net-hours (hours:minutes) } \\
\text { Captures (number) } \\
\text { Captures per net-hour }\end{array}$} & 5:00 & 5:00 & 5:00 & 5:00 & 5:00 & 5:00 & $5: 10$ & 5:00 & $5: 00$ & $5: 00$ & $50: 10$ \\
\hline & & & 3 & 2 & 1 & 5 & 1 & 1 & 0 & 3 & 2 & 1 & 19 \\
\hline & & & 0.60 & 0.40 & 0.20 & 1.00 & 0.20 & 0.20 & 0.00 & 0.60 & 0.40 & 0.20 & 0.38 \\
\hline \multirow[b]{3}{*}{8} & \multirow[b]{3}{*}{$7-14-2015$} & \multirow{3}{*}{$\begin{array}{l}\text { Net-hours (hours:minutes) } \\
\text { Captures (number) } \\
\text { Captures per net-hour }\end{array}$} & 5:00 & $5: 10$ & $5: 10$ & 5:00 & 5:00 & 5:00 & $5: 10$ & $5: 00$ & $5: 00$ & $5: 00$ & $50: 30$ \\
\hline & & & 0 & 4 & 3 & 0 & 1 & 0 & 1 & 0 & 2 & 2 & 13 \\
\hline & & & 0.00 & 0.77 & 0.58 & 0.00 & 0.20 & 0.00 & 0.19 & 0.00 & 0.40 & 0.40 & 0.26 \\
\hline & & Net-hours (hours:minutes) & 5:00 & 5:00 & 5:00 & 5:00 & $5: 10$ & $5: 10$ & 5:00 & 5:00 & $4: 50$ & 5:00 & $50: 10$ \\
\hline & & Captures (number) & 1 & 0 & 0 & 0 & 2 & 0 & 0 & 2 & 1 & 1 & 7 \\
\hline 9 & $7-28-2015$ & Captures per net-hour & 0.20 & 0.00 & 0.00 & 0.00 & 0.39 & 0.00 & 0.00 & 0.40 & 0.21 & 0.20 & 0.66 \\
\hline
\end{tabular}




\begin{tabular}{|c|c|c|c|c|c|c|c|c|c|c|c|c|c|}
\hline \multirow{2}{*}{$\begin{array}{l}\text { MAPS } \\
\text { period }\end{array}$} & \multirow[b]{2}{*}{ Date } & & \multicolumn{10}{|c|}{ Net } & \multirow{2}{*}{$\begin{array}{c}\text { Total } \\
\text { (hours:minutes }\end{array}$} \\
\hline & & & 1 & 2 & 3 & 4 & 5 & 6 & 7 & 8 & 9 & 10 & \\
\hline \multirow[b]{3}{*}{10} & \multirow[b]{3}{*}{$8-4-2015$} & \multirow{3}{*}{$\begin{array}{l}\text { Net-hours (hours:minutes) } \\
\text { Captures (number) } \\
\text { Captures per net-hour }\end{array}$} & $5: 10$ & $5: 00$ & $5: 00$ & $5: 00$ & $4: 50$ & $5: 00$ & $5: 00$ & $5: 10$ & $5: 10$ & $5: 20$ & $50: 40$ \\
\hline & & & 4 & 4 & 1 & 0 & 1 & 0 & 1 & 0 & 6 & 6 & 23 \\
\hline & & & 0.77 & 0.80 & 0.20 & 0.00 & 0.21 & 0.00 & 0.20 & 0.00 & 1.16 & 1.13 & 0.45 \\
\hline \multirow{3}{*}{\multicolumn{2}{|c|}{ Totals by net }} & \multirow{3}{*}{$\begin{array}{l}\text { Net-hours (hours:minutes) } \\
\text { Captures (number) } \\
\text { Captures per net-hour }\end{array}$} & $50: 40$ & $50: 20$ & $50: 20$ & $49: 50$ & $47: 30$ & $50: 00$ & $50: 00$ & $50: 10$ & $50: 10$ & $51: 10$ & $500: 10$ \\
\hline & & & 43 & 36 & 20 & 30 & 18 & 18 & 16 & 33 & 41 & 49 & $305^{1}$ \\
\hline & & & 0.85 & 0.72 & 0.40 & 0.60 & 0.38 & 0.36 & 0.32 & 0.66 & 0.82 & 0.96 & 0.61 \\
\hline
\end{tabular}

${ }^{1}$ Total includes one bird captured with no net number recorded. 
Table C8. Capture frequency of individuals during Monitoring Avian Productivity and Survivorship, Point Loma banding station, San Diego, California, 2011.

[See appendix A for bird species codes. Species in italics are non-breeding neotropical migrants]

\begin{tabular}{|c|c|c|c|c|c|c|}
\hline \multirow{2}{*}{$\begin{array}{l}\text { Species } \\
\text { code }\end{array}$} & \multicolumn{3}{|c|}{$\begin{array}{l}\text { Number of individuals per capture } \\
\text { incidence (banded birds only) }\end{array}$} & \multicolumn{3}{|c|}{ Total number of individuals } \\
\hline & 1 capture & 2 captures & 3 captures & Banded birds & Unbanded birds & All birds \\
\hline CAQU & 0 & 0 & 0 & 0 & 1 & 1 \\
\hline ANHU & 0 & 0 & 0 & 0 & 55 & 55 \\
\hline $\mathrm{COHU}$ & 0 & 0 & 0 & 0 & 5 & 5 \\
\hline RUHU & 0 & 0 & 0 & 0 & 1 & 1 \\
\hline ALHU & 0 & 0 & 0 & 0 & 2 & 2 \\
\hline USHU & 0 & 0 & 0 & 0 & 1 & 1 \\
\hline UNHU & 0 & 0 & 0 & 0 & 4 & 4 \\
\hline WEWP & 3 & 0 & 0 & 3 & 0 & 3 \\
\hline WIFL & 2 & 0 & 0 & 2 & 0 & 2 \\
\hline DUFL & 1 & 0 & 0 & 1 & 0 & 1 \\
\hline PSFL & 11 & 0 & 0 & 11 & 1 & 12 \\
\hline COFL & 1 & 0 & 0 & 1 & 0 & 1 \\
\hline ATFL & 2 & 1 & 0 & 3 & 0 & 3 \\
\hline WAVI & 21 & 1 & 0 & 22 & 0 & 22 \\
\hline WESJ & 1 & 0 & 0 & 1 & 0 & 1 \\
\hline $\mathrm{BUSH}$ & 39 & 4 & 1 & 44 & 2 & 46 \\
\hline BEWR & 13 & 2 & 0 & 15 & 1 & 16 \\
\hline HOWR & 2 & 0 & 0 & 2 & 0 & 2 \\
\hline SWTH & 8 & 0 & 0 & 8 & 0 & 8 \\
\hline HETH & 1 & 0 & 0 & 1 & 0 & 1 \\
\hline WREN & 20 & 0 & 0 & 20 & 0 & 20 \\
\hline NOMO & 3 & 0 & 0 & 3 & 0 & 3 \\
\hline CATH & 12 & 0 & 0 & 12 & 0 & 12 \\
\hline PHAI & 1 & 0 & 0 & 1 & 0 & 1 \\
\hline OCWA & 67 & 5 & 1 & 73 & 1 & 74 \\
\hline YEWA & 3 & 0 & 0 & 3 & 0 & 3 \\
\hline$B T Y W$ & 1 & 0 & 0 & 1 & 0 & 1 \\
\hline TOWA & 13 & 0 & 0 & 13 & 0 & 13 \\
\hline THWH & 2 & 0 & 0 & 2 & 0 & 2 \\
\hline HEWA & 2 & 0 & 0 & 2 & 0 & 2 \\
\hline$M G W A$ & 1 & 0 & 0 & 1 & 0 & 1 \\
\hline WIWA & 43 & 0 & 0 & 43 & 0 & 43 \\
\hline $\mathrm{YBCH}$ & 1 & 0 & 0 & 1 & 0 & 1 \\
\hline WETA & 3 & 0 & 0 & 3 & 0 & 3 \\
\hline SPTO & 26 & 0 & 0 & 26 & 1 & 27 \\
\hline CALT & 26 & 4 & 0 & 30 & 0 & 30 \\
\hline CHSP & 1 & 0 & 0 & 1 & 1 & 2 \\
\hline SOSP & 1 & 0 & 0 & 1 & 0 & 1 \\
\hline WTSP & 1 & 0 & 0 & 1 & 0 & 1 \\
\hline$B H G R$ & 0 & 0 & 0 & 0 & 1 & 1 \\
\hline$B L G R$ & 1 & 0 & 0 & 1 & 0 & 1 \\
\hline
\end{tabular}




\begin{tabular}{ccccccc}
\hline & \multicolumn{2}{c}{$\begin{array}{c}\text { Number of individuals per capture } \\
\text { incidence (banded birds only) }\end{array}$} & \multicolumn{3}{c}{ Total number of individuals } \\
\cline { 2 - 7 } $\begin{array}{c}\text { Species } \\
\text { code }\end{array}$ & 1 capture & 2 captures & 3 captures & Banded birds & Unbanded birds & All birds \\
\hline LAZB & 3 & 0 & 0 & 3 & 0 & 3 \\
\hline OROR & 1 & 0 & 0 & 1 & 0 & 1 \\
HOOR & 13 & 0 & 0 & 13 & 0 & 13 \\
BUOR & 2 & 0 & 0 & 2 & 0 & 2 \\
HOFI & 7 & 0 & 0 & 7 & 1 & 8 \\
LEGO & 11 & 0 & 0 & 11 & 0 & 11 \\
AMGO & 1 & 0 & 0 & 1 & 0 & 1 \\
\hline Total & 371 & 17 & 2 & 390 & 78 & 468 \\
\hline
\end{tabular}


Table C9. Capture frequency of individuals during Monitoring Avian Productivity and Survivorship, Point Loma banding station, San Diego, California, 2012.

[See appendix A for bird species codes. Species in italics are non-breeding neotropical migrants]

\begin{tabular}{|c|c|c|c|c|c|c|}
\hline \multirow[b]{2}{*}{$\begin{array}{l}\text { Species } \\
\text { code }\end{array}$} & \multicolumn{3}{|c|}{$\begin{array}{l}\text { Number of individuals per capture } \\
\text { incidence (banded birds only) }\end{array}$} & \multicolumn{3}{|c|}{ Total number of individuals } \\
\hline & 1 capture & 2 captures & 3 captures & Banded birds & Unbanded birds & All birds \\
\hline $\mathrm{BCHU}$ & 0 & 0 & 0 & 0 & 2 & 2 \\
\hline ANHU & 0 & 0 & 0 & 0 & 46 & 46 \\
\hline $\mathrm{COHU}$ & 0 & 0 & 0 & 0 & 1 & 1 \\
\hline CAHU & 0 & 0 & 0 & 0 & 1 & 1 \\
\hline RUHU & 0 & 0 & 0 & 0 & 4 & 4 \\
\hline$A L H U$ & 0 & 0 & 0 & 0 & 2 & 2 \\
\hline USHU & 0 & 0 & 0 & 0 & 2 & 2 \\
\hline WEWP & 5 & 0 & 0 & 5 & 0 & 5 \\
\hline WIFL & 3 & 0 & 0 & 3 & 0 & 3 \\
\hline PSFL & 32 & 0 & 0 & 32 & 0 & 32 \\
\hline ATFL & 5 & 0 & 0 & 5 & 0 & 5 \\
\hline WAVI & 16 & 0 & 0 & 16 & 0 & 16 \\
\hline WESJ & 2 & 0 & 0 & 2 & 0 & 2 \\
\hline BUSH & 43 & 3 & 1 & 47 & 0 & 47 \\
\hline BEWR & 14 & 0 & 0 & 14 & 0 & 14 \\
\hline SWTH & 4 & 0 & 0 & 4 & 0 & 4 \\
\hline WREN & 8 & 0 & 0 & 8 & 0 & 8 \\
\hline CATH & 5 & 0 & 0 & 5 & 0 & 5 \\
\hline OCWA & 25 & 1 & 0 & 26 & 0 & 26 \\
\hline AUWA & 1 & 0 & 0 & 1 & 0 & 1 \\
\hline TOWA & 7 & 0 & 0 & 7 & 0 & 7 \\
\hline HEWA & 4 & 0 & 0 & 4 & 0 & 4 \\
\hline COYE & 1 & 0 & 0 & 1 & 0 & 1 \\
\hline WIWA & 44 & 0 & 0 & 44 & 0 & 44 \\
\hline WETA & 2 & 0 & 0 & 2 & 0 & 2 \\
\hline SPTO & 10 & 1 & 0 & 11 & 1 & 12 \\
\hline CALT & 8 & 1 & 0 & 9 & 0 & 9 \\
\hline SOSP & 4 & 0 & 0 & 4 & 0 & 4 \\
\hline$B L G R$ & 1 & 0 & 0 & 1 & 0 & 1 \\
\hline LAZB & 2 & 0 & 0 & 2 & 0 & 2 \\
\hline$I N B U$ & 1 & 0 & 0 & 1 & 0 & 1 \\
\hline HOOR & 10 & 0 & 0 & 10 & 0 & 10 \\
\hline HOFI & 3 & 0 & 0 & 3 & 0 & 3 \\
\hline Total & 260 & 6 & 1 & 267 & 59 & 326 \\
\hline
\end{tabular}


Table C10. Capture frequency of individuals during Monitoring Avian Productivity and Survivorship, Point Loma banding station, San Diego, California, 2013.

[See appendix A for bird species codes. Species in italics are non-breeding neotropical migrants]

\begin{tabular}{|c|c|c|c|c|c|c|}
\hline \multirow[b]{2}{*}{ Species code } & \multicolumn{3}{|c|}{$\begin{array}{l}\text { Number of individuals per capture } \\
\text { incidence (banded birds only) }\end{array}$} & \multicolumn{3}{|c|}{ Total number of individuals } \\
\hline & 1 capture & 2 captures & 3 captures & Banded birds & Unbanded birds & All birds \\
\hline ANHU & 0 & 0 & 0 & 0 & 46 & 46 \\
\hline $\mathrm{COHU}$ & 0 & 0 & 0 & 0 & 2 & 2 \\
\hline RUHU & 0 & 0 & 0 & 0 & 1 & 1 \\
\hline$A L H U$ & 0 & 0 & 0 & 0 & 7 & 7 \\
\hline USHU & 0 & 0 & 0 & 0 & 1 & 1 \\
\hline WEWP & 2 & 0 & 0 & 2 & 0 & 2 \\
\hline WIFL & 2 & 0 & 0 & 2 & 0 & 2 \\
\hline HAFL & 1 & 0 & 0 & 1 & 0 & 1 \\
\hline GRFL & 1 & 0 & 0 & 1 & 0 & 1 \\
\hline PSFL & 13 & 0 & 0 & 13 & 0 & 13 \\
\hline COFL & 1 & 0 & 0 & 1 & 0 & 1 \\
\hline ATFL & 3 & 0 & 0 & 3 & 0 & 3 \\
\hline LBVI & 2 & 0 & 0 & 2 & 0 & 2 \\
\hline CAVI & 1 & 0 & 0 & 1 & 0 & 1 \\
\hline WAVI & 7 & 0 & 0 & 7 & 0 & 7 \\
\hline REVI & 1 & 0 & 0 & 1 & 0 & 1 \\
\hline BUSH & 28 & 5 & 0 & 33 & 0 & 33 \\
\hline BEWR & 12 & 3 & 0 & 15 & 1 & 16 \\
\hline HOWR & 1 & 0 & 0 & 1 & 0 & 1 \\
\hline CAGN & 1 & 0 & 0 & 1 & 0 & 1 \\
\hline SWTH & 2 & 0 & 0 & 2 & 0 & 2 \\
\hline WREN & 9 & 0 & 0 & 9 & 0 & 9 \\
\hline NOMO & 1 & 0 & 0 & 1 & 0 & 1 \\
\hline CATH & 3 & 0 & 0 & 3 & 0 & 3 \\
\hline OCWA & 29 & 0 & 0 & 29 & 0 & 29 \\
\hline TOWA & 1 & 0 & 0 & 1 & 0 & 1 \\
\hline HEWA & 2 & 0 & 0 & 2 & 0 & 2 \\
\hline MGWA & 1 & 0 & 0 & 1 & 0 & 1 \\
\hline WIWA & 6 & 0 & 0 & 6 & 0 & 6 \\
\hline WETA & 3 & 0 & 0 & 3 & 0 & 3 \\
\hline SPTO & 25 & 0 & 1 & 26 & 0 & 26 \\
\hline CALT & 7 & 0 & 0 & 7 & 1 & 8 \\
\hline BHGR & 2 & 0 & 0 & 2 & 0 & 2 \\
\hline HOOR & 8 & 0 & 0 & 8 & 0 & 8 \\
\hline HOFI & 11 & 0 & 0 & 11 & 0 & 11 \\
\hline LEGO & 2 & 0 & 0 & 2 & 0 & 2 \\
\hline Total & 188 & 8 & 1 & 197 & 59 & 256 \\
\hline
\end{tabular}


Table C11. Capture frequency of individuals during Monitoring Avian Productivity and Survivorship, Point Loma banding station, San Diego, California, 2014.

[See appendix A for bird species codes. Species in italics are non-breeding neotropical migrants]

\begin{tabular}{|c|c|c|c|c|c|}
\hline \multirow{2}{*}{$\begin{array}{l}\text { Species } \\
\text { code }\end{array}$} & \multicolumn{2}{|c|}{$\begin{array}{l}\text { Number of individuals per capture } \\
\text { incidence (banded birds only) }\end{array}$} & \multicolumn{3}{|c|}{ Total number of individuals } \\
\hline & 1 capture & 2 captures & Banded birds & Unbanded birds & All birds \\
\hline $\mathrm{BCHU}$ & 0 & 0 & 0 & 1 & 1 \\
\hline ANHU & 0 & 0 & 0 & 56 & 56 \\
\hline $\mathrm{COHU}$ & 0 & 0 & 0 & 8 & 8 \\
\hline $\mathrm{CAHU}$ & 0 & 0 & 0 & 2 & 2 \\
\hline$A L H U$ & 0 & 0 & 0 & 11 & 11 \\
\hline USHU & 0 & 0 & 0 & 1 & 1 \\
\hline DOWO & 1 & 0 & 1 & 0 & 1 \\
\hline WEWP & 1 & 0 & 1 & 0 & 1 \\
\hline WIFL & 2 & 0 & 2 & 0 & 2 \\
\hline HAFL & 2 & 0 & 2 & 0 & 2 \\
\hline PSFL & 74 & 0 & 74 & 0 & 74 \\
\hline ATFL & 3 & 0 & 3 & 0 & 3 \\
\hline LBVI & 1 & 0 & 1 & 0 & 1 \\
\hline WAVI & 31 & 0 & 31 & 0 & 31 \\
\hline BUSH & 9 & 1 & 10 & 0 & 10 \\
\hline BEWR & 9 & 0 & 9 & 1 & 10 \\
\hline SWTH & 8 & 0 & 8 & 0 & 8 \\
\hline HETH & 1 & 0 & 1 & 0 & 1 \\
\hline WREN & 13 & 1 & 14 & 0 & 14 \\
\hline NOMO & 1 & 0 & 1 & 0 & 1 \\
\hline CATH & 2 & 0 & 2 & 0 & 2 \\
\hline OCWA & 12 & 0 & 12 & 0 & 12 \\
\hline YEWA & 5 & 0 & 5 & 0 & 5 \\
\hline TOWA & 8 & 0 & 8 & 0 & 8 \\
\hline HEWA & 2 & 0 & 2 & 1 & 3 \\
\hline MGWA & 7 & 0 & 7 & 0 & 7 \\
\hline WIWA & 26 & 0 & 26 & 1 & 27 \\
\hline WETA & 3 & 0 & 3 & 0 & 3 \\
\hline SPTO & 12 & 0 & 12 & 0 & 12 \\
\hline CALT & 9 & 1 & 10 & 0 & 10 \\
\hline SAGS & 1 & 0 & 1 & 0 & 1 \\
\hline ORJU & 1 & 0 & 1 & 0 & 1 \\
\hline HOOR & 5 & 0 & 5 & 0 & 5 \\
\hline $\mathrm{HOFI}$ & 4 & 0 & 4 & 0 & 4 \\
\hline Total & 253 & 3 & 256 & 82 & 338 \\
\hline
\end{tabular}


Table C12. Capture frequency of individuals during Monitoring Avian Productivity and Survivorship, Point Loma banding station, San Diego, California, 2015.

[See appendix A for bird species codes. Species in italics are non-breeding neotropical migrants]

\begin{tabular}{|c|c|c|c|c|c|}
\hline \multirow[b]{2}{*}{$\begin{array}{l}\text { Species } \\
\text { code }\end{array}$} & \multicolumn{2}{|c|}{$\begin{array}{l}\text { Number of individuals per capture } \\
\text { incidence (banded birds only) }\end{array}$} & \multicolumn{3}{|c|}{ Total number of individuals } \\
\hline & 1 capture & 2 captures & Banded birds & Unbanded birds & All birds \\
\hline AMKE & 0 & 0 & 0 & 1 & 1 \\
\hline MODO & 0 & 0 & 0 & 1 & 1 \\
\hline ANHU & 0 & 0 & 0 & 8 & 8 \\
\hline $\mathrm{COHU}$ & 0 & 0 & 0 & 3 & 3 \\
\hline CAHU & 0 & 0 & 0 & 2 & 2 \\
\hline ALHU & 0 & 0 & 0 & 3 & 3 \\
\hline WEWP & 5 & 0 & 5 & 0 & 5 \\
\hline WIFL & 3 & 0 & 3 & 0 & 3 \\
\hline HAFL & 1 & 0 & 1 & 0 & 1 \\
\hline PSFL & 30 & 0 & 30 & 1 & 31 \\
\hline ATFL & 2 & 0 & 2 & 0 & 2 \\
\hline CAKI & 1 & 0 & 1 & 0 & 1 \\
\hline WAVI & 24 & 1 & 25 & 0 & 25 \\
\hline BARS & 1 & 0 & 1 & 0 & 1 \\
\hline BUSH & 18 & 0 & 18 & 0 & 18 \\
\hline BEWR & 26 & 4 & 30 & 1 & 31 \\
\hline RCKI & 1 & 0 & 1 & 0 & 1 \\
\hline CAGN & 1 & 0 & 1 & 0 & 1 \\
\hline WREN & 21 & 3 & 24 & 1 & 25 \\
\hline GRCA & 1 & 0 & 1 & 0 & 1 \\
\hline NOMO & 1 & 0 & 1 & 0 & 1 \\
\hline CATH & 7 & 0 & 7 & 0 & 7 \\
\hline OCWA & 16 & 1 & 17 & 1 & 18 \\
\hline YEWA & 5 & 0 & 5 & 0 & 5 \\
\hline TOWA & 5 & 0 & 5 & 0 & 5 \\
\hline HEWA & 1 & 0 & 1 & 0 & 1 \\
\hline WIWA & 31 & 0 & 31 & 0 & 31 \\
\hline CAWA & 1 & 0 & 1 & 0 & 1 \\
\hline WETA & 3 & 0 & 3 & 0 & 3 \\
\hline SPTO & 23 & 1 & 24 & 1 & 25 \\
\hline CALT & 17 & 2 & 19 & 1 & 20 \\
\hline LAZB & 1 & 0 & 1 & 0 & 1 \\
\hline HOOR & 3 & 0 & 3 & 0 & 3 \\
\hline HOFI & 1 & 0 & 1 & 0 & 1 \\
\hline LEGO & 6 & 0 & 6 & 0 & 6 \\
\hline Total & 256 & 12 & 268 & 24 & 292 \\
\hline
\end{tabular}


Table C13. Number of captures by date during Monitoring Avian Productivity and Survivorship (MAPS), Point Loma banding station, San Diego, California, 2011.

[See appendix A for bird species codes. Species in italics are non-breeding neotropical migrants. Unknown species not included in species total. Includes multiple captures of some individuals]

\begin{tabular}{|c|c|c|c|c|c|c|c|c|c|c|c|c|}
\hline \multirow[b]{4}{*}{ Species code } & \multicolumn{10}{|c|}{ MAPS period } & \multirow[b]{4}{*}{ Total } & \multirow[b]{4}{*}{$\begin{array}{c}\text { Captures per } 10 \\
\text { net-hours } \\
\text { (486:20:00 total } \\
\text { net-hours) }\end{array}$} \\
\hline & 1 & 2 & 3 & 4 & 5 & 6 & 7 & 8 & 9 & 10 & & \\
\hline & \multicolumn{10}{|c|}{ Date } & & \\
\hline & 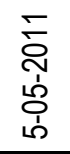 & 容 & 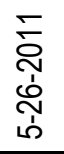 & 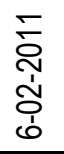 & 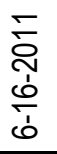 & $\begin{array}{l}\text { Г్ } \\
\text { సे } \\
\text { ஸे }\end{array}$ & 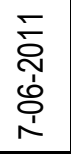 & 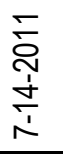 & $\frac{\check{c}}{\stackrel{T}{\grave{N}}}$ & 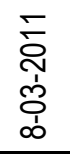 & & \\
\hline CAQU & 1 & 0 & 0 & 0 & 0 & 0 & 0 & 0 & 0 & 0 & 1 & 0.21 \\
\hline ANHU & 5 & 14 & 9 & 16 & 3 & 1 & 3 & 3 & 1 & 0 & 55 & 11.32 \\
\hline $\mathrm{COHU}$ & 1 & 1 & 0 & 1 & 0 & 1 & 0 & 1 & 0 & 0 & 5 & 1.03 \\
\hline RUHU & 0 & 0 & 0 & 1 & 0 & 0 & 0 & 0 & 0 & 0 & 1 & 0.21 \\
\hline ALHU & 0 & 0 & 0 & 0 & 0 & 0 & 1 & 0 & 0 & 1 & 2 & 0.41 \\
\hline USHU & 0 & 1 & 0 & 0 & 0 & 0 & 0 & 0 & 0 & 0 & 1 & 0.21 \\
\hline UNHU & 1 & 0 & 1 & 0 & 0 & 1 & 1 & 0 & 0 & 0 & 4 & 0.82 \\
\hline WEWP & 0 & 1 & 1 & 1 & 0 & 0 & 0 & 0 & 0 & 0 & 3 & 0.62 \\
\hline WIFL & 0 & 0 & 2 & 0 & 0 & 0 & 0 & 0 & 0 & 0 & 2 & 0.41 \\
\hline DUFL & 0 & 0 & 0 & 0 & 0 & 1 & 0 & 0 & 0 & 0 & 1 & 0.21 \\
\hline PSFL & 1 & 10 & 1 & 0 & 0 & 0 & 0 & 0 & 0 & 0 & 12 & 2.47 \\
\hline COFL & 0 & 0 & 1 & 0 & 0 & 0 & 0 & 0 & 0 & 0 & 1 & 0.21 \\
\hline ATFL & 0 & 0 & 1 & 1 & 1 & 1 & 0 & 0 & 0 & 0 & 4 & 0.82 \\
\hline WAVI & 2 & 8 & 10 & 3 & 0 & 0 & 0 & 0 & 0 & 0 & 23 & 4.73 \\
\hline WESJ & 0 & 0 & 0 & 0 & 0 & 1 & 0 & 0 & 0 & 0 & 1 & 0.21 \\
\hline $\mathrm{BUSH}$ & 0 & 6 & 1 & 6 & 0 & 9 & 6 & 10 & 9 & 6 & 53 & 10.91 \\
\hline BEWR & 0 & 3 & 1 & 0 & 0 & 0 & 7 & 3 & 2 & 2 & 18 & 3.70 \\
\hline HOWR & 0 & 0 & 0 & 0 & 0 & 0 & 0 & 1 & 0 & 1 & 2 & 0.41 \\
\hline SWTH & 2 & 6 & 0 & 0 & 0 & 0 & 0 & 0 & 0 & 0 & 8 & 1.65 \\
\hline HETH & 1 & 0 & 0 & 0 & 0 & 0 & 0 & 0 & 0 & 0 & 1 & 0.21 \\
\hline WREN & 0 & 6 & 1 & 2 & 4 & 0 & 3 & 3 & 1 & 0 & 20 & 4.12 \\
\hline NOMO & 0 & 0 & 0 & 0 & 2 & 0 & 1 & 0 & 0 & 0 & 3 & 0.62 \\
\hline CATH & 1 & 1 & 1 & 1 & 1 & 1 & 0 & 3 & 0 & 3 & 12 & 2.47 \\
\hline PHAI & 1 & 0 & 0 & 0 & 0 & 0 & 0 & 0 & 0 & 0 & 1 & 0.21 \\
\hline OCWA & 20 & 15 & 20 & 8 & 7 & 2 & 2 & 2 & 1 & 4 & 81 & 16.67 \\
\hline YEWA & 1 & 0 & 2 & 0 & 0 & 0 & 0 & 0 & 0 & 0 & 3 & 0.62 \\
\hline BTYW & 0 & 1 & 0 & 0 & 0 & 0 & 0 & 0 & 0 & 0 & 1 & 0.21 \\
\hline TOWA & 2 & 10 & 1 & 0 & 0 & 0 & 0 & 0 & 0 & 0 & 13 & 2.67 \\
\hline$T H W H$ & 1 & 1 & 0 & 0 & 0 & 0 & 0 & 0 & 0 & 0 & 2 & 0.41 \\
\hline HEWA & 0 & 2 & 0 & 0 & 0 & 0 & 0 & 0 & 0 & 0 & 2 & 0.41 \\
\hline MGWA & 0 & 0 & 1 & 0 & 0 & 0 & 0 & 0 & 0 & 0 & 1 & 0.21 \\
\hline WIWA & 11 & 24 & 7 & 1 & 0 & 0 & 0 & 0 & 0 & 0 & 43 & 8.85 \\
\hline $\mathrm{YBCH}$ & 1 & 0 & 0 & 0 & 0 & 0 & 0 & 0 & 0 & 0 & 1 & 0.21 \\
\hline WETA & 0 & 3 & 0 & 0 & 0 & 0 & 0 & 0 & 0 & 0 & 3 & 0.62 \\
\hline SPTO & 4 & 1 & 1 & 2 & 1 & 3 & 8 & 1 & 3 & 3 & 27 & 5.56 \\
\hline CALT & 4 & 1 & 4 & 2 & 4 & 6 & 4 & 2 & 5 & 2 & 34 & 7.00 \\
\hline
\end{tabular}




\begin{tabular}{|c|c|c|c|c|c|c|c|c|c|c|c|c|}
\hline \multirow[b]{4}{*}{ Species code } & \multicolumn{10}{|c|}{ MAPS period } & \multirow[b]{4}{*}{ Total } & \multirow[b]{4}{*}{$\begin{array}{l}\text { Captures per } 100 \\
\text { net-hours } \\
\text { (486:20:00 total } \\
\text { net-hours) }\end{array}$} \\
\hline & 1 & 2 & 3 & 4 & 5 & 6 & 7 & 8 & 9 & 10 & & \\
\hline & \multicolumn{10}{|c|}{ Date } & & \\
\hline & 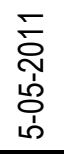 & 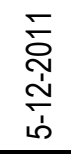 & 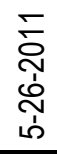 & 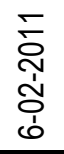 & 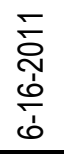 & $\begin{array}{l}\overline{\check{\sigma}} \\
\text { ஸे } \\
0\end{array}$ & 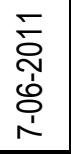 & 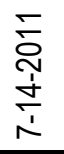 & $\frac{\overleftarrow{c}}{\stackrel{T}{N}}$ & 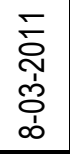 & & \\
\hline CHSP & 1 & 0 & 1 & 0 & 0 & 0 & 0 & 0 & 0 & 0 & 2 & 0.41 \\
\hline SOSP & 0 & 0 & 0 & 1 & 0 & 0 & 0 & 0 & 0 & 0 & 1 & 0.21 \\
\hline WTSP & 1 & 0 & 0 & 0 & 0 & 0 & 0 & 0 & 0 & 0 & 1 & 0.21 \\
\hline$B H G R$ & 0 & 0 & 0 & 0 & 0 & 0 & 0 & 1 & 0 & 0 & 1 & 0.21 \\
\hline$B L G R$ & 0 & 0 & 0 & 0 & 1 & 0 & 0 & 0 & 0 & 0 & 1 & 0.21 \\
\hline LAZB & 3 & 0 & 0 & 0 & 0 & 0 & 0 & 0 & 0 & 0 & 3 & 0.62 \\
\hline OROR & 0 & 0 & 0 & 0 & 0 & 1 & 0 & 0 & 0 & 0 & 1 & 0.21 \\
\hline HOOR & 0 & 3 & 0 & 0 & 0 & 1 & 7 & 0 & 2 & 0 & 13 & 2.67 \\
\hline BUOR & 0 & 1 & 0 & 0 & 0 & 0 & 1 & 0 & 0 & 0 & 2 & 0.41 \\
\hline $\mathrm{HOFI}$ & 0 & 0 & 2 & 2 & 0 & 0 & 2 & 1 & 0 & 1 & 8 & 1.65 \\
\hline LEGO & 2 & 0 & 0 & 2 & 3 & 3 & 1 & 0 & 0 & 0 & 11 & 2.26 \\
\hline AMGO & 0 & 0 & 0 & 0 & 1 & 0 & 0 & 0 & 0 & 0 & 1 & 0.21 \\
\hline Captures per day & 67 & 119 & 69 & 50 & 28 & 32 & 47 & 31 & 24 & 23 & 490 & 100.82 \\
\hline Total species & 20 & 20 & 20 & 16 & 11 & 13 & 13 & 12 & 8 & 9 & 45 & 9.26 \\
\hline
\end{tabular}


Table C14. Number of captures by date during Monitoring Avian Productivity and Survivorship (MAPS), Point Loma banding station, San Diego, California, 2012.

[See appendix A for bird species codes. Species in italics are non-breeding neotropical migrants. Unknown species not included in species total. Includes multiple captures of some individuals]

\begin{tabular}{|c|c|c|c|c|c|c|c|c|c|c|c|c|}
\hline & \multicolumn{10}{|c|}{ MAPS period } & \multirow[b]{4}{*}{ Total } & \multirow[b]{4}{*}{$\begin{array}{c}\text { Captures per } 100 \\
\text { net-hours } \\
\text { (501:00:00 total net- } \\
\text { hours) }\end{array}$} \\
\hline & 1 & 2 & 3 & 4 & 5 & 6 & 7 & 8 & 9 & 10 & & \\
\hline & \multicolumn{10}{|c|}{ Date } & & \\
\hline Species code & 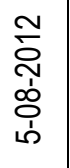 & 䒞 & $\begin{array}{l}\text { N } \\
\text { స̦ } \\
\text { సे } \\
\text { ஸ่ }\end{array}$ & 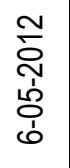 & 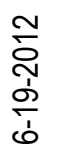 & $\begin{array}{l}\text { ָे } \\
\text { సे } \\
\text { ஸे } \\
\text { ô }\end{array}$ & 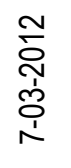 & $\begin{array}{l}\text { N } \\
\text { ஸे } \\
\frac{1}{1}\end{array}$ & 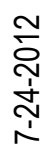 & 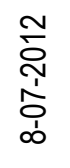 & & \\
\hline $\mathrm{BCHU}$ & 0 & 1 & 0 & 0 & 0 & 0 & 0 & 0 & 1 & 0 & 2 & 0.40 \\
\hline ANHU & 9 & 9 & 3 & 0 & 4 & 6 & 0 & 8 & 3 & 4 & 46 & 9.18 \\
\hline $\mathrm{COHU}$ & 0 & 0 & 0 & 0 & 1 & 0 & 0 & 0 & 0 & 0 & 1 & 0.20 \\
\hline CAHU & 1 & 0 & 0 & 0 & 0 & 0 & 0 & 0 & 0 & 0 & 1 & 0.20 \\
\hline RUHU & 0 & 1 & 0 & 0 & 1 & 0 & 0 & 2 & 0 & 0 & 4 & 0.80 \\
\hline ALHU & 0 & 0 & 0 & 0 & 0 & 1 & 0 & 1 & 0 & 0 & 2 & 0.40 \\
\hline USHU & 0 & 0 & 0 & 1 & 0 & 0 & 0 & 0 & 1 & 0 & 2 & 0.40 \\
\hline WEWP & 0 & 2 & 3 & 0 & 0 & 0 & 0 & 0 & 0 & 0 & 5 & 1.00 \\
\hline WIFL & 0 & 0 & 1 & 2 & 0 & 0 & 0 & 0 & 0 & 0 & 3 & 0.60 \\
\hline PSFL & 4 & 16 & 8 & 4 & 0 & 0 & 0 & 0 & 0 & 0 & 32 & 6.39 \\
\hline ATFL & 0 & 0 & 2 & 1 & 0 & 1 & 0 & 0 & 1 & 0 & 5 & 1.00 \\
\hline WAVI & 6 & 9 & 1 & 0 & 0 & 0 & 0 & 0 & 0 & 0 & 16 & 3.19 \\
\hline WESJ & 0 & 0 & 0 & 0 & 1 & 0 & 1 & 0 & 0 & 0 & 2 & 0.40 \\
\hline $\mathrm{BUSH}$ & 6 & 0 & 1 & 5 & 1 & 9 & 1 & 5 & 16 & 8 & 52 & 10.38 \\
\hline BEWR & 1 & 2 & 3 & 2 & 1 & 1 & 2 & 2 & 0 & 0 & 14 & 2.79 \\
\hline SWTH & 1 & 3 & 0 & 0 & 0 & 0 & 0 & 0 & 0 & 0 & 4 & 0.80 \\
\hline WREN & 0 & 0 & 0 & 0 & 1 & 0 & 1 & 1 & 4 & 1 & 8 & 1.60 \\
\hline CATH & 1 & 1 & 0 & 0 & 0 & 0 & 0 & 1 & 2 & 0 & 5 & 1.00 \\
\hline OCWA & 9 & 5 & 4 & 5 & 0 & 1 & 0 & 0 & 3 & 0 & 27 & 5.39 \\
\hline AUWA & 1 & 0 & 0 & 0 & 0 & 0 & 0 & 0 & 0 & 0 & 1 & 0.20 \\
\hline TOWA & 6 & 1 & 0 & 0 & 0 & 0 & 0 & 0 & 0 & 0 & 7 & 1.40 \\
\hline HEWA & 4 & 0 & 0 & 0 & 0 & 0 & 0 & 0 & 0 & 0 & 4 & 0.80 \\
\hline COYE & 0 & 0 & 0 & 0 & 0 & 0 & 0 & 0 & 1 & 0 & 1 & 0.20 \\
\hline WIWA & 34 & 7 & 3 & 0 & 0 & 0 & 0 & 0 & 0 & 0 & 44 & 8.78 \\
\hline WETA & 0 & 1 & 0 & 0 & 0 & 0 & 0 & 0 & 1 & 0 & 2 & 0.40 \\
\hline SPTO & 0 & 0 & 2 & 2 & 2 & 0 & 3 & 1 & 1 & 2 & 13 & 2.59 \\
\hline CALT & 2 & 2 & 0 & 0 & 2 & 2 & 0 & 0 & 0 & 2 & 10 & 2.00 \\
\hline SOSP & 1 & 2 & 0 & 0 & 0 & 0 & 1 & 0 & 0 & 0 & 4 & 0.80 \\
\hline$B L G R$ & 0 & 0 & 0 & 0 & 1 & 0 & 0 & 0 & 0 & 0 & 1 & 0.20 \\
\hline LAZB & 1 & 1 & 0 & 0 & 0 & 0 & 0 & 0 & 0 & 0 & 2 & 0.40 \\
\hline INBU & 0 & 0 & 1 & 0 & 0 & 0 & 0 & 0 & 0 & 0 & 1 & 0.20 \\
\hline HOOR & 0 & 0 & 0 & 1 & 0 & 3 & 0 & 4 & 0 & 2 & 10 & 2.00 \\
\hline HOFI & 1 & 0 & 0 & 1 & 0 & 0 & 0 & 1 & 0 & 0 & 3 & 0.60 \\
\hline Captures per day & 88 & 63 & 32 & 24 & 15 & 24 & 9 & 26 & 34 & 19 & 334 & 66.67 \\
\hline Total species & 17 & 16 & 12 & 9 & 10 & 8 & 6 & 10 & 10 & 6 & 32 & 6.39 \\
\hline
\end{tabular}


Table C15. Number of captures by date during Monitoring Avian Productivity and Survivorship (MAPS), Point Loma banding station, San Diego, California, 2013.

[See appendix A for bird species codes. Species in italics are non-breeding neotropical migrants. Unknown species not included in species total. Includes multiple captures of some individuals]

\begin{tabular}{|c|c|c|c|c|c|c|c|c|c|c|c|c|}
\hline \multirow[b]{4}{*}{ Species code } & \multicolumn{10}{|c|}{ MAPS period } & \multirow[b]{4}{*}{ Total } & \multirow[b]{4}{*}{$\begin{array}{c}\text { Captures per } \\
100 \text { net-hours } \\
\text { (499:00:00 total } \\
\text { net-hours) }\end{array}$} \\
\hline & 1 & 2 & 3 & 4 & 5 & 6 & 7 & 8 & 9 & 10 & & \\
\hline & \multicolumn{10}{|c|}{ Date } & & \\
\hline & 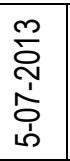 & 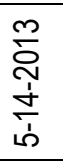 & 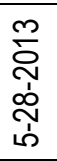 & 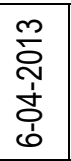 & 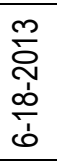 & 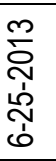 & 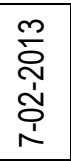 & 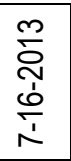 & $\begin{array}{l}\text { ஸे } \\
\text { ஸे } \\
\text { ஸे }\end{array}$ & 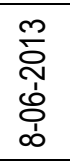 & & \\
\hline ANHU & 10 & 6 & 5 & 11 & 5 & 3 & 1 & 2 & 1 & 2 & 46 & 9.22 \\
\hline $\mathrm{COHU}$ & 0 & 1 & 0 & 0 & 0 & 1 & 0 & 0 & 0 & 0 & 2 & 0.40 \\
\hline RUHU & 0 & 0 & 0 & 0 & 0 & 0 & 0 & 1 & 0 & 0 & 1 & 0.20 \\
\hline$A L H U$ & 1 & 2 & 0 & 0 & 2 & 1 & 0 & 1 & 0 & 0 & 7 & 1.40 \\
\hline USHU & 0 & 0 & 0 & 0 & 0 & 0 & 0 & 1 & 0 & 0 & 1 & 0.20 \\
\hline WEWP & 0 & 2 & 0 & 0 & 0 & 0 & 0 & 0 & 0 & 0 & 2 & 0.40 \\
\hline WIFL & 0 & 0 & 0 & 1 & 0 & 0 & 1 & 0 & 0 & 0 & 2 & 0.40 \\
\hline HAFL & 0 & 1 & 0 & 0 & 0 & 0 & 0 & 0 & 0 & 0 & 1 & 0.20 \\
\hline GRFL & 0 & 1 & 0 & 0 & 0 & 0 & 0 & 0 & 0 & 0 & 1 & 0.20 \\
\hline PSFL & 0 & 3 & 6 & 4 & 0 & 0 & 0 & 0 & 0 & 0 & 13 & 2.61 \\
\hline COFL & 0 & 1 & 0 & 0 & 0 & 0 & 0 & 0 & 0 & 0 & 1 & 0.20 \\
\hline ATFL & 0 & 1 & 0 & 0 & 1 & 1 & 0 & 0 & 0 & 0 & 3 & 0.60 \\
\hline$\angle B V I$ & 0 & 0 & 0 & 0 & 0 & 0 & 0 & 0 & 1 & 1 & 2 & 0.40 \\
\hline CAVI & 0 & 1 & 0 & 0 & 0 & 0 & 0 & 0 & 0 & 0 & 1 & 0.20 \\
\hline WAVI & 0 & 6 & 1 & 0 & 0 & 0 & 0 & 0 & 0 & 0 & 7 & 1.40 \\
\hline REVI & 0 & 0 & 0 & 0 & 0 & 0 & 0 & 0 & 1 & 0 & 1 & 0.20 \\
\hline $\mathrm{BUSH}$ & 9 & 0 & 1 & 1 & 7 & 1 & 1 & 7 & 11 & 1 & 39 & 7.82 \\
\hline BEWR & 2 & 1 & 2 & 2 & 2 & 3 & 1 & 2 & 4 & 0 & 19 & 3.81 \\
\hline HOWR & 0 & 0 & 0 & 0 & 0 & 0 & 0 & 1 & 0 & 0 & 1 & 0.20 \\
\hline CAGN & 0 & 0 & 0 & 0 & 0 & 0 & 0 & 0 & 1 & 0 & 1 & 0.20 \\
\hline SWTH & 0 & 2 & 0 & 0 & 0 & 0 & 0 & 0 & 0 & 0 & 2 & 0.40 \\
\hline WREN & 0 & 0 & 0 & 2 & 1 & 1 & 0 & 3 & 0 & 2 & 9 & 1.80 \\
\hline NOMO & 0 & 0 & 0 & 0 & 0 & 0 & 0 & 0 & 1 & 0 & 1 & 0.20 \\
\hline CATH & 0 & 1 & 1 & 0 & 0 & 0 & 1 & 0 & 0 & 0 & 3 & 0.60 \\
\hline OCWA & 3 & 2 & 10 & 6 & 1 & 3 & 0 & 0 & 0 & 4 & 29 & 5.81 \\
\hline TOWA & 1 & 0 & 0 & 0 & 0 & 0 & 0 & 0 & 0 & 0 & 1 & 0.20 \\
\hline HEWA & 0 & 2 & 0 & 0 & 0 & 0 & 0 & 0 & 0 & 0 & 2 & 0.40 \\
\hline MGWA & 0 & 1 & 0 & 0 & 0 & 0 & 0 & 0 & 0 & 0 & 1 & 0.20 \\
\hline WIWA & 0 & 6 & 0 & 0 & 0 & 0 & 0 & 0 & 0 & 0 & 6 & 1.20 \\
\hline WETA & 0 & 3 & 0 & 0 & 0 & 0 & 0 & 0 & 0 & 0 & 3 & 0.60 \\
\hline SPTO & 1 & 0 & 3 & 2 & 0 & 5 & 8 & 6 & 1 & 2 & 28 & 5.61 \\
\hline CALT & 0 & 0 & 2 & 1 & 2 & 1 & 0 & 1 & 0 & 1 & 8 & 1.60 \\
\hline BHGR & 1 & 1 & 0 & 0 & 0 & 0 & 0 & 0 & 0 & 0 & 2 & 0.40 \\
\hline HOOR & 1 & 1 & 0 & 0 & 1 & 0 & 1 & 4 & 0 & 0 & 8 & 1.60 \\
\hline $\mathrm{HOFI}$ & 4 & 7 & 0 & 0 & 0 & 0 & 0 & 0 & 0 & 0 & 11 & 2.20 \\
\hline LEGO & 1 & 0 & 0 & 0 & 1 & 0 & 0 & 0 & 0 & 0 & 2 & 0.40 \\
\hline
\end{tabular}




\begin{tabular}{|c|c|c|c|c|c|c|c|c|c|c|c|c|}
\hline \multirow[b]{4}{*}{ Species code } & \multicolumn{10}{|c|}{ MAPS period } & \multirow[b]{4}{*}{ Total } & \multirow[b]{4}{*}{$\begin{array}{c}\text { Captures per } \\
100 \text { net-hours } \\
\text { (499:00:00 total } \\
\text { net-hours) }\end{array}$} \\
\hline & 1 & 2 & 3 & 4 & 5 & 6 & 7 & 8 & 9 & 10 & & \\
\hline & \multicolumn{10}{|c|}{ Date } & & \\
\hline & 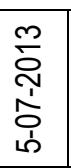 & 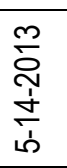 & $\begin{array}{l}\text { m } \\
\stackrel{N}{~} \\
\text { ò } \\
\text { ஸे }\end{array}$ & 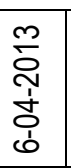 & 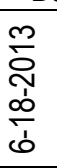 & 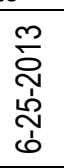 & 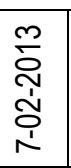 & 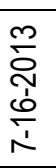 & $\begin{array}{l}\text { ָे } \\
\text { ஸे } \\
\text { ते }\end{array}$ & 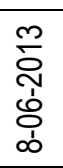 & & \\
\hline Captures per day & 34 & 52 & 31 & 30 & 23 & 20 & 14 & 29 & 21 & 13 & 267 & 53.51 \\
\hline Total species & 11 & 22 & 9 & 9 & 10 & 10 & 7 & 10 & 8 & 7 & 35 & 7.01 \\
\hline
\end{tabular}


Table C16. Number of captures by date during Monitoring Avian Productivity and Survivorship (MAPS), Point Loma banding station, San Diego, California, 2014.

[See appendix A for bird species codes. Species in italics are non-breeding neotropical migrants. Includes multiple captures of some individuals. Unknown species not included in species total]

\begin{tabular}{|c|c|c|c|c|c|c|c|c|c|c|c|c|}
\hline \multirow[b]{4}{*}{ Species code } & \multicolumn{10}{|c|}{ MAPS period } & \multirow[b]{4}{*}{ Total } & \multirow[b]{4}{*}{$\begin{array}{c}\text { Captures per } \\
100 \text { net-hours } \\
\text { (497:40:00 total- } \\
\text { net hours) }\end{array}$} \\
\hline & 1 & 2 & 3 & 4 & 5 & 6 & 7 & 8 & 9 & 10 & & \\
\hline & \multicolumn{10}{|c|}{ Date } & & \\
\hline & \begin{tabular}{l}
$\forall$ \\
\multirow{2}{*}{} \\
ò \\
ம்
\end{tabular} & 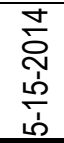 & 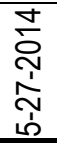 & 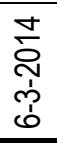 & 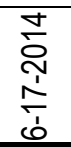 & 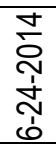 & 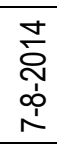 & 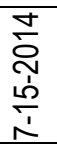 & 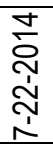 & 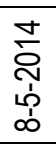 & & \\
\hline $\mathrm{BCHU}$ & 0 & 1 & 0 & 0 & 0 & 0 & 0 & 0 & 0 & 0 & 1 & 0.20 \\
\hline ANHU & 24 & 9 & 7 & 6 & 4 & 1 & 3 & 0 & 1 & 1 & 56 & 11.25 \\
\hline $\mathrm{COHU}$ & 1 & 1 & 3 & 0 & 0 & 0 & 0 & 2 & 1 & 0 & 8 & 1.61 \\
\hline CAHU & 2 & 0 & 0 & 0 & 0 & 0 & 0 & 0 & 0 & 0 & 2 & 0.40 \\
\hline$A L H U$ & 1 & 1 & 1 & 1 & 0 & 3 & 2 & 1 & 1 & 0 & 11 & 2.21 \\
\hline USHU & 0 & 0 & 1 & 0 & 0 & 0 & 0 & 0 & 0 & 0 & 1 & 0.20 \\
\hline DOWO & 0 & 1 & 0 & 0 & 0 & 0 & 0 & 0 & 0 & 0 & 1 & 0.20 \\
\hline WEWP & 0 & 0 & 1 & 0 & 0 & 0 & 0 & 0 & 0 & 0 & 1 & 0.20 \\
\hline WIFL & 0 & 0 & 1 & 1 & 0 & 0 & 0 & 0 & 0 & 0 & 2 & 0.40 \\
\hline HAFL & 0 & 2 & 0 & 0 & 0 & 0 & 0 & 0 & 0 & 0 & 2 & 0.40 \\
\hline PSFL & 3 & 13 & 56 & 1 & 0 & 0 & 0 & 0 & 1 & 0 & 74 & 14.87 \\
\hline ATFL & 0 & 0 & 2 & 1 & 0 & 0 & 0 & 0 & 0 & 0 & 3 & 0.60 \\
\hline LBVI & 0 & 0 & 1 & 0 & 0 & 0 & 0 & 0 & 0 & 0 & 1 & 0.20 \\
\hline WAVI & 21 & 2 & 7 & 1 & 0 & 0 & 0 & 0 & 0 & 0 & 31 & 6.23 \\
\hline $\mathrm{BUSH}$ & 3 & 0 & 0 & 0 & 5 & 0 & 0 & 1 & 2 & 0 & 11 & 2.21 \\
\hline BEWR & 2 & 1 & 4 & 1 & 0 & 0 & 0 & 1 & 0 & 1 & 10 & 2.01 \\
\hline SWTH & 3 & 2 & 3 & 0 & 0 & 0 & 0 & 0 & 0 & 0 & 8 & 1.61 \\
\hline HETH & 0 & 1 & 0 & 0 & 0 & 0 & 0 & 0 & 0 & 0 & 1 & 0.20 \\
\hline WREN & 3 & 4 & 0 & 0 & 1 & 2 & 3 & 0 & 0 & 2 & 15 & 3.01 \\
\hline NOMO & 0 & 0 & 0 & 0 & 1 & 0 & 0 & 0 & 0 & 0 & 1 & 0.20 \\
\hline CATH & 0 & 0 & 0 & 0 & 1 & 0 & 1 & 0 & 0 & 0 & 2 & 0.40 \\
\hline OCWA & 2 & 1 & 2 & 1 & 2 & 1 & 1 & 1 & 0 & 1 & 12 & 2.41 \\
\hline YEWA & 0 & 5 & 0 & 0 & 0 & 0 & 0 & 0 & 0 & 0 & 5 & 1.00 \\
\hline TOWA & 3 & 5 & 0 & 0 & 0 & 0 & 0 & 0 & 0 & 0 & 8 & 1.61 \\
\hline HEWA & 2 & 1 & 0 & 0 & 0 & 0 & 0 & 0 & 0 & 0 & 3 & 0.60 \\
\hline MGWA & 0 & 7 & 0 & 0 & 0 & 0 & 0 & 0 & 0 & 0 & 7 & 1.41 \\
\hline WIWA & 12 & 15 & 0 & 0 & 0 & 0 & 0 & 0 & 0 & 0 & 27 & 5.43 \\
\hline WETA & 1 & 2 & 0 & 0 & 0 & 0 & 0 & 0 & 0 & 0 & 3 & 0.60 \\
\hline SPTO & 4 & 0 & 2 & 2 & 2 & 0 & 2 & 0 & 0 & 0 & 12 & 2.41 \\
\hline CALT & 1 & 0 & 1 & 0 & 3 & 2 & 3 & 0 & 0 & 1 & 11 & 2.21 \\
\hline SAGS & 0 & 1 & 0 & 0 & 0 & 0 & 0 & 0 & 0 & 0 & 1 & 0.20 \\
\hline ORJU & 0 & 0 & 0 & 0 & 0 & 0 & 1 & 0 & 0 & 0 & 1 & 0.20 \\
\hline HOOR & 2 & 0 & 0 & 0 & 0 & 0 & 1 & 0 & 2 & 0 & 5 & 1.00 \\
\hline $\mathrm{HOFI}$ & 2 & 0 & 1 & 0 & 0 & 1 & 0 & 0 & 0 & 0 & 4 & 0.80 \\
\hline Captures per day & 92 & 75 & 93 & 15 & 19 & 10 & 17 & 6 & 8 & 6 & 341 & 68.52 \\
\hline Total species & 19 & 20 & 15 & 9 & 8 & 6 & 9 & 5 & 6 & 5 & 33 & 6.63 \\
\hline
\end{tabular}


Table C17. Number of captures by date during Monitoring Avian Productivity and Survivorship (MAPS), Point Loma banding station, San Diego, California, 2015.

[See appendix A for bird species codes. Species in italics are non-breeding neotropical migrants. Includes multiple captures of some individuals]

\begin{tabular}{|c|c|c|c|c|c|c|c|c|c|c|c|c|}
\hline \multirow[b]{4}{*}{ Species code } & \multicolumn{10}{|c|}{ MAPS period } & \multirow[b]{4}{*}{ Total } & \multirow{4}{*}{$\begin{array}{c}\text { Captures per } \\
100 \text { net-hours } \\
\text { (500:10:00 } \\
\text { total net- } \\
\text { hours) }\end{array}$} \\
\hline & 1 & 2 & 3 & 4 & 5 & 6 & 7 & 8 & 9 & 10 & & \\
\hline & \multicolumn{10}{|c|}{ Date } & & \\
\hline & 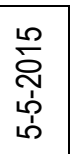 & 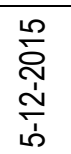 & 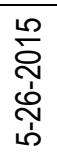 & 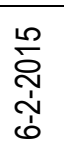 & 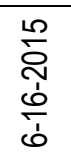 & $\begin{array}{l}\text { م) } \\
\text { ஸे } \\
\text { ஸे } \\
0\end{array}$ & $\begin{array}{l}\frac{n}{2} \\
\frac{N}{1} \\
\end{array}$ & 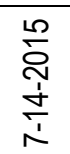 & 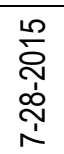 & 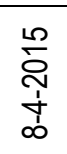 & & \\
\hline AMKE & 1 & 0 & 0 & 0 & 0 & 0 & 0 & 0 & 0 & 0 & 1 & 0.20 \\
\hline MODO & 0 & 0 & 0 & 1 & 0 & 0 & 0 & 0 & 0 & 0 & 1 & 0.20 \\
\hline ANHU & 3 & 2 & 1 & 0 & 0 & 0 & 0 & 1 & 0 & 1 & 8 & 1.60 \\
\hline $\mathrm{COHU}$ & 0 & 1 & 0 & 1 & 0 & 1 & 0 & 0 & 0 & 0 & 3 & 0.60 \\
\hline CAHU & 0 & 2 & 0 & 0 & 0 & 0 & 0 & 0 & 0 & 0 & 2 & 0.40 \\
\hline$A L H U$ & 0 & 3 & 0 & 0 & 0 & 0 & 0 & 0 & 0 & 0 & 3 & 0.60 \\
\hline WEWP & 0 & 0 & 5 & 0 & 0 & 0 & 0 & 0 & 0 & 0 & 5 & 1.00 \\
\hline WIFL & 0 & 0 & 3 & 0 & 0 & 0 & 0 & 0 & 0 & 0 & 3 & 0.60 \\
\hline HAFL & 0 & 1 & 0 & 0 & 0 & 0 & 0 & 0 & 0 & 0 & 1 & 0.20 \\
\hline PSFL & 5 & 9 & 17 & 0 & 0 & 0 & 0 & 0 & 0 & 0 & 31 & 6.20 \\
\hline ATFL & 0 & 1 & 0 & 1 & 0 & 0 & 0 & 0 & 0 & 0 & 2 & 0.40 \\
\hline CAKI & 1 & 0 & 0 & 0 & 0 & 0 & 0 & 0 & 0 & 0 & 1 & 0.20 \\
\hline WAVI & 16 & 9 & 1 & 0 & 0 & 0 & 0 & 0 & 0 & 0 & 26 & 5.20 \\
\hline BARS & 0 & 0 & 0 & 0 & 0 & 0 & 1 & 0 & 0 & 0 & 1 & 0.20 \\
\hline $\mathrm{BUSH}$ & 1 & 4 & 0 & 6 & 0 & 0 & 0 & 0 & 0 & 7 & 18 & 3.60 \\
\hline BEWR & 5 & 6 & 5 & 2 & 3 & 8 & 1 & 0 & 2 & 3 & 35 & 7.00 \\
\hline$R C K I$ & 1 & 0 & 0 & 0 & 0 & 0 & 0 & 0 & 0 & 0 & 1 & 0.20 \\
\hline CAGN & 0 & 1 & 0 & 0 & 0 & 0 & 0 & 0 & 0 & 0 & 1 & 0.20 \\
\hline WREN & 1 & 4 & 2 & 2 & 4 & 4 & 6 & 3 & 1 & 1 & 28 & 5.60 \\
\hline GRCA & 1 & 0 & 0 & 0 & 0 & 0 & 0 & 0 & 0 & 0 & 1 & 0.20 \\
\hline NOMO & 0 & 0 & 0 & 0 & 0 & 0 & 1 & 0 & 0 & 0 & 1 & 0.20 \\
\hline CATH & 0 & 0 & 1 & 0 & 1 & 0 & 1 & 3 & 1 & 0 & 7 & 1.40 \\
\hline OCWA & 7 & 2 & 1 & 3 & 0 & 1 & 0 & 0 & 1 & 4 & 19 & 3.80 \\
\hline YEWA & 0 & 2 & 3 & 0 & 0 & 0 & 0 & 0 & 0 & 0 & 5 & 1.00 \\
\hline TOWA & 3 & 2 & 0 & 0 & 0 & 0 & 0 & 0 & 0 & 0 & 5 & 1.00 \\
\hline HEWA & 0 & 1 & 0 & 0 & 0 & 0 & 0 & 0 & 0 & 0 & 1 & 0.20 \\
\hline WIWA & 20 & 10 & 1 & 0 & 0 & 0 & 0 & 0 & 0 & 0 & 31 & 6.20 \\
\hline CAWA & 0 & 0 & 1 & 0 & 0 & 0 & 0 & 0 & 0 & 0 & 1 & 0.20 \\
\hline WETA & 0 & 3 & 0 & 0 & 0 & 0 & 0 & 0 & 0 & 0 & 3 & 0.60 \\
\hline SPTO & 4 & 4 & 3 & 0 & 3 & 5 & 4 & 4 & 0 & 0 & 27 & 5.40 \\
\hline CALT & 1 & 2 & 6 & 1 & 2 & 2 & 5 & 1 & 1 & 1 & 22 & 4.40 \\
\hline LAZB & 0 & 0 & 0 & 0 & 0 & 0 & 0 & 0 & 1 & 0 & 1 & 0.20 \\
\hline HOOR & 1 & 0 & 0 & 0 & 0 & 0 & 0 & 1 & 0 & 1 & 3 & 0.60 \\
\hline $\mathrm{HOFI}$ & 0 & 0 & 0 & 0 & 0 & 1 & 0 & 0 & 0 & 0 & 1 & 0.20 \\
\hline LEGO & 0 & 0 & 0 & 0 & 1 & 0 & 0 & 0 & 0 & 5 & 6 & 1.20 \\
\hline Captures per day & 71 & 69 & 50 & 17 & 14 & 22 & 19 & 13 & 7 & 23 & 305 & 60.98 \\
\hline Total Species & 16 & 20 & 14 & 8 & 6 & 7 & 7 & 6 & 6 & 8 & 35 & 7.01 \\
\hline
\end{tabular}


Table C18. Sex and age of individual birds (banded and unbanded) captured during Monitoring Avian Productivity and Survivorship (MAPS), Point Loma banding station, San Diego, California, 2011.

[See appendix A for bird species codes. Species in italics are non-breeding neotropical migrants. Age: HY=hatching-year, AHY=after-hatching-year, SY=second-year, ASY=after-secondyear, l=indeterminable age]

\begin{tabular}{|c|c|c|c|c|c|c|c|c|c|c|c|c|c|c|c|c|c|c|c|}
\hline \multirow{3}{*}{$\begin{array}{l}\text { Species } \\
\text { code }\end{array}$} & \multirow{2}{*}{\multicolumn{5}{|c|}{$\begin{array}{c}\text { Female } \\
\text { Age }\end{array}$}} & \multirow{3}{*}{$\begin{array}{c}\text { Female } \\
\text { total }\end{array}$} & \multirow{2}{*}{\multicolumn{5}{|c|}{$\begin{array}{l}\text { Male } \\
\text { Age }\end{array}$}} & \multirow{3}{*}{$\begin{array}{l}\text { Male } \\
\text { total }\end{array}$} & \multirow{2}{*}{\multicolumn{5}{|c|}{$\begin{array}{c}\text { Unknown sex } \\
\text { Age }\end{array}$}} & \multirow{3}{*}{$\begin{array}{c}\text { Unknown } \\
\text { total }\end{array}$} & \multirow{3}{*}{$\begin{array}{c}\text { Species } \\
\text { total }\end{array}$} \\
\hline & & & & & & & & & & & & & & & & & & & \\
\hline & HY & AHY & SY & ASY & $I$ & & HY & AHY & SY & ASY & I & & HY & AHY & SY & ASY & I & & \\
\hline CAQU & 0 & 1 & 0 & 0 & 0 & 1 & 0 & 0 & 0 & 0 & 0 & 0 & 0 & 0 & 0 & 0 & 0 & 0 & 1 \\
\hline ANHU & 5 & 6 & 0 & 0 & 6 & 17 & 16 & 5 & 0 & 0 & 4 & 25 & 4 & 0 & 0 & 0 & 9 & 13 & 55 \\
\hline $\mathrm{COHU}$ & 0 & 0 & 0 & 0 & 0 & 0 & 1 & 2 & 0 & 0 & 0 & 3 & 0 & 0 & 0 & 0 & 2 & 2 & 5 \\
\hline RUHU & 1 & 0 & 0 & 0 & 0 & 1 & 0 & 0 & 0 & 0 & 0 & 0 & 0 & 0 & 0 & 0 & 0 & 0 & 1 \\
\hline$A L H U$ & 0 & 1 & 0 & 0 & 1 & 2 & 0 & 0 & 0 & 0 & 0 & 0 & 0 & 0 & 0 & 0 & 0 & 0 & 2 \\
\hline USHU & 0 & 0 & 0 & 0 & 0 & 0 & 0 & 0 & 0 & 0 & 0 & 0 & 0 & 0 & 0 & 0 & 1 & 1 & 1 \\
\hline UNHU & 0 & 0 & 0 & 0 & 0 & 0 & 0 & 0 & 0 & 0 & 0 & 0 & 1 & 0 & 0 & 0 & 3 & 4 & 4 \\
\hline WEWP & 0 & 0 & 0 & 0 & 0 & 0 & 0 & 0 & 0 & 0 & 0 & 0 & 0 & 3 & 0 & 0 & 0 & 3 & 3 \\
\hline WIFL & 0 & 0 & 0 & 0 & 0 & 0 & 0 & 0 & 0 & 0 & 0 & 0 & 0 & 1 & 0 & 1 & 0 & 2 & 2 \\
\hline DUFL & 0 & 0 & 0 & 0 & 0 & 0 & 0 & 0 & 0 & 0 & 0 & 0 & 0 & 0 & 0 & 0 & 1 & 1 & 1 \\
\hline COFL & 0 & 0 & 0 & 0 & 0 & 0 & 0 & 0 & 0 & 0 & 0 & 0 & 0 & 1 & 0 & 0 & 0 & 1 & 1 \\
\hline$P S F L$ & 0 & 0 & 0 & 0 & 0 & 0 & 0 & 0 & 0 & 0 & 0 & 0 & 0 & 10 & 1 & 0 & 1 & 12 & 12 \\
\hline ATFL & 0 & 0 & 0 & 0 & 0 & 0 & 0 & 0 & 0 & 0 & 0 & 0 & 0 & 0 & 3 & 0 & 0 & 3 & 3 \\
\hline WAVI & 0 & 0 & 0 & 0 & 0 & 0 & 0 & 0 & 0 & 0 & 0 & 0 & 0 & 20 & 2 & 0 & 0 & 22 & 22 \\
\hline WESJ & 0 & 0 & 0 & 0 & 0 & 0 & 0 & 0 & 0 & 0 & 0 & 0 & 0 & 1 & 0 & 0 & 0 & 1 & 1 \\
\hline $\mathrm{BUSH}$ & 11 & 7 & 2 & 1 & 0 & 21 & 8 & 7 & 1 & 1 & 0 & 17 & 5 & 0 & 1 & 0 & 2 & 8 & 46 \\
\hline BEWR & 0 & 1 & 0 & 0 & 0 & 1 & 0 & 0 & 0 & 0 & 0 & 0 & 12 & 3 & 0 & 0 & 0 & 15 & 16 \\
\hline HOWR & 0 & 0 & 0 & 0 & 0 & 0 & 0 & 0 & 0 & 0 & 0 & 0 & 1 & 0 & 0 & 0 & 1 & 2 & 2 \\
\hline SWTH & 0 & 0 & 0 & 0 & 0 & 0 & 0 & 0 & 0 & 0 & 0 & 0 & 0 & 5 & 1 & 2 & 0 & 8 & 8 \\
\hline HETH & 0 & 0 & 0 & 0 & 0 & 0 & 0 & 0 & 0 & 0 & 0 & 0 & 0 & 1 & 0 & 0 & 0 & 1 & 1 \\
\hline WREN & 0 & 0 & 0 & 0 & 0 & 0 & 0 & 0 & 0 & 0 & 0 & 0 & 13 & 6 & 0 & 1 & 0 & 20 & 20 \\
\hline NOMO & 0 & 0 & 0 & 1 & 0 & 1 & 0 & 0 & 0 & 0 & 0 & 0 & 1 & 0 & 0 & 1 & 0 & 2 & 3 \\
\hline CATH & 0 & 0 & 0 & 0 & 0 & 0 & 0 & 0 & 0 & 0 & 0 & 0 & 7 & 5 & 0 & 0 & 0 & 12 & 12 \\
\hline PHAI & 0 & 0 & 1 & 0 & 0 & 1 & 0 & 0 & 0 & 0 & 0 & 0 & 0 & 0 & 0 & 0 & 0 & 0 & 1 \\
\hline OCWA & 0 & 8 & 10 & 10 & 0 & 28 & 0 & 5 & 6 & 9 & 0 & 20 & 17 & 8 & 0 & 0 & 1 & 26 & 74 \\
\hline
\end{tabular}




\begin{tabular}{|c|c|c|c|c|c|c|c|c|c|c|c|c|c|c|c|c|c|c|c|}
\hline \multirow{3}{*}{$\begin{array}{l}\text { Species } \\
\text { code }\end{array}$} & \multirow{2}{*}{\multicolumn{5}{|c|}{$\begin{array}{l}\text { Female } \\
\text { Age }\end{array}$}} & \multirow{3}{*}{$\begin{array}{c}\text { Female } \\
\text { total }\end{array}$} & \multirow{2}{*}{\multicolumn{5}{|c|}{$\begin{array}{l}\text { Male } \\
\text { Age } \\
\end{array}$}} & \multirow{3}{*}{$\begin{array}{l}\text { Male } \\
\text { total }\end{array}$} & \multirow{2}{*}{\multicolumn{5}{|c|}{$\begin{array}{c}\text { Unknown sex } \\
\text { Age }\end{array}$}} & \multirow{3}{*}{$\begin{array}{c}\text { Unknown } \\
\text { total }\end{array}$} & \multirow{3}{*}{$\begin{array}{c}\text { Species } \\
\text { total }\end{array}$} \\
\hline & & & & & & & & & & & & & & & & & & & \\
\hline & HY & AHY & SY & ASY & 1 & & HY & AHY & SY & ASY & $\mathrm{I}$ & & HY & AHY & sY & ASY & 1 & & \\
\hline YEWA & 0 & 0 & 1 & 0 & 0 & 1 & 0 & 0 & 1 & 1 & 0 & 2 & 0 & 0 & 0 & 0 & 0 & 0 & 3 \\
\hline BTYW & 0 & 0 & 1 & 0 & 0 & 1 & 0 & 0 & 0 & 0 & 0 & 0 & 0 & 0 & 0 & 0 & 0 & 0 & 1 \\
\hline TOWA & 0 & 0 & 4 & 2 & 0 & 6 & 0 & 0 & 0 & 6 & 0 & 6 & 0 & 1 & 0 & 0 & 0 & 1 & 13 \\
\hline $\mathrm{THWH}$ & 0 & 1 & 0 & 1 & 0 & 2 & 0 & 0 & 0 & 0 & 0 & 0 & 0 & 0 & 0 & 0 & 0 & 0 & 2 \\
\hline HEWA & 0 & 0 & 0 & 1 & 0 & 1 & 0 & 0 & 1 & 0 & 0 & 1 & 0 & 0 & 0 & 0 & 0 & 0 & 2 \\
\hline MGWA & 0 & 0 & 0 & 0 & 0 & 0 & 0 & 0 & 0 & 0 & 0 & 0 & 0 & 1 & 0 & 0 & 0 & 1 & 1 \\
\hline WIWA & 0 & 3 & 8 & 5 & 0 & 16 & 0 & 3 & 3 & 2 & 0 & 8 & 0 & 13 & 1 & 5 & 0 & 19 & 43 \\
\hline$Y B C H$ & 0 & 1 & 0 & 0 & 0 & 1 & 0 & 0 & 0 & 0 & 0 & 0 & 0 & 0 & 0 & 0 & 0 & 0 & 1 \\
\hline WETA & 0 & 0 & 0 & 2 & 0 & 2 & 0 & 0 & 0 & 1 & 0 & 1 & 0 & 0 & 0 & 0 & 0 & 0 & 3 \\
\hline SPTO & 0 & 2 & 1 & 2 & 0 & 5 & 0 & 2 & 0 & 5 & 0 & 7 & 14 & 0 & 0 & 0 & 1 & 15 & 27 \\
\hline CALT & 0 & 2 & 0 & 0 & 0 & 2 & 0 & 8 & 0 & 0 & 0 & 8 & 16 & 3 & 1 & 0 & 0 & 20 & 30 \\
\hline CHSP & 0 & 0 & 0 & 0 & 0 & 0 & 0 & 0 & 0 & 0 & 0 & 0 & 0 & 0 & 1 & 0 & 1 & 2 & 2 \\
\hline SOSP & 0 & 0 & 0 & 0 & 0 & 0 & 0 & 1 & 0 & 0 & 0 & 1 & 0 & 0 & 0 & 0 & 0 & 0 & 1 \\
\hline WTSP & 0 & 0 & 0 & 0 & 0 & 0 & 0 & 0 & 0 & 0 & 0 & 0 & 0 & 1 & 0 & 0 & 0 & 1 & 1 \\
\hline BHGR & 0 & 0 & 0 & 0 & 0 & 0 & 0 & 1 & 0 & 0 & 0 & 1 & 0 & 0 & 0 & 0 & 0 & 0 & 1 \\
\hline$B L G R$ & 0 & 0 & 1 & 0 & 0 & 1 & 0 & 0 & 0 & 0 & 0 & 0 & 0 & 0 & 0 & 0 & 0 & 0 & 1 \\
\hline LAZB & 0 & 0 & 0 & 1 & 0 & 1 & 0 & 0 & 0 & 2 & 0 & 2 & 0 & 0 & 0 & 0 & 0 & 0 & 3 \\
\hline OROR & 0 & 0 & 0 & 0 & 0 & 0 & 0 & 0 & 1 & 0 & 0 & 1 & 0 & 0 & 0 & 0 & 0 & 0 & 1 \\
\hline HOOR & 0 & 2 & 1 & 0 & 0 & 3 & 0 & 1 & 0 & 0 & 0 & 1 & 8 & 0 & 1 & 0 & 0 & 9 & 13 \\
\hline BUOR & 0 & 0 & 0 & 0 & 0 & 0 & 0 & 1 & 0 & 0 & 0 & 1 & 1 & 0 & 0 & 0 & 0 & 1 & 2 \\
\hline $\mathrm{HOFI}$ & 0 & 1 & 0 & 0 & 0 & 1 & 0 & 3 & 0 & 0 & 0 & 3 & 3 & 0 & 0 & 0 & 1 & 4 & 8 \\
\hline LEGO & 6 & 3 & 1 & 0 & 0 & 10 & 0 & 0 & 1 & 0 & 0 & 1 & 0 & 0 & 0 & 0 & 0 & 0 & 11 \\
\hline AMGO & 0 & 0 & 0 & 0 & 0 & 0 & 1 & 0 & 0 & 0 & 0 & 1 & 0 & 0 & 0 & 0 & 0 & 0 & 1 \\
\hline Total & 23 & 39 & 31 & 26 & 7 & 126 & 26 & 39 & 14 & 27 & 4 & 110 & 103 & 83 & 12 & 10 & 24 & 232 & 468 \\
\hline
\end{tabular}


Table C19. Sex and age of individual birds (banded and unbanded) captured during Monitoring Avian Productivity and Survivorship (MAPS), Point Loma banding station, San Diego, California, 2012.

[See appendix A for bird species codes. Species in italics are non-breeding neotropical migrants. Age: HY=hatching-year, AHY=after-hatching-year, SY=second-year, ASY=after-secondyear, l=indeterminable age]

\begin{tabular}{|c|c|c|c|c|c|c|c|c|c|c|c|c|c|c|c|c|c|c|c|}
\hline \multirow{3}{*}{$\begin{array}{c}\text { Species } \\
\text { code }\end{array}$} & \multirow{2}{*}{\multicolumn{5}{|c|}{$\begin{array}{c}\text { Female } \\
\text { Age }\end{array}$}} & \multirow[b]{3}{*}{ Female total } & \multirow{2}{*}{\multicolumn{5}{|c|}{$\begin{array}{l}\text { Male } \\
\text { Age }\end{array}$}} & \multirow[b]{3}{*}{ Male total } & \multirow{2}{*}{\multicolumn{5}{|c|}{$\begin{array}{c}\text { Unknown sex } \\
\text { Age }\end{array}$}} & \multirow{3}{*}{$\begin{array}{c}\text { Unknown } \\
\text { total }\end{array}$} & \multirow{3}{*}{$\begin{array}{c}\text { Species } \\
\text { total }\end{array}$} \\
\hline & & & & & & & & & & & & & & & & & & & \\
\hline & HY & AHY & SY & ASY & I & & HY & AHY & SY & ASY & I & & HY & AHY & SY & ASY & 1 & & \\
\hline $\mathrm{BCHU}$ & 0 & 2 & 0 & 0 & 0 & 2 & 0 & 0 & 0 & 0 & 0 & 0 & 0 & 0 & 0 & 0 & 0 & 0 & 2 \\
\hline ANHU & 8 & 8 & 0 & 0 & 2 & 18 & 17 & 5 & 0 & 0 & 3 & 25 & 0 & 0 & 0 & 0 & 3 & 3 & 46 \\
\hline $\mathrm{COHU}$ & 1 & 0 & 0 & 0 & 0 & 1 & 0 & 0 & 0 & 0 & 0 & 0 & 0 & 0 & 0 & 0 & 0 & 0 & 1 \\
\hline CAHU & 0 & 1 & 0 & 0 & 0 & 1 & 0 & 0 & 0 & 0 & 0 & 0 & 0 & 0 & 0 & 0 & 0 & 0 & 1 \\
\hline RUHU & 0 & 2 & 0 & 0 & 0 & 2 & 0 & 1 & 0 & 0 & 0 & 1 & 0 & 1 & 0 & 0 & 0 & 1 & 4 \\
\hline$A L H U$ & 0 & 1 & 0 & 0 & 0 & 1 & 0 & 1 & 0 & 0 & 0 & 1 & 0 & 0 & 0 & 0 & 0 & 0 & 2 \\
\hline USHU & 0 & 0 & 0 & 0 & 0 & 0 & 0 & 0 & 0 & 0 & 0 & 0 & 1 & 1 & 0 & 0 & 0 & 2 & 2 \\
\hline WEWP & 0 & 0 & 0 & 0 & 0 & 0 & 0 & 0 & 0 & 0 & 0 & 0 & 0 & 5 & 0 & 0 & 0 & 5 & 5 \\
\hline WIFL & 0 & 0 & 0 & 0 & 0 & 0 & 0 & 0 & 0 & 0 & 0 & 0 & 0 & 2 & 1 & 0 & 0 & 3 & 3 \\
\hline PSFL & 0 & 0 & 0 & 0 & 0 & 0 & 0 & 0 & 0 & 0 & 0 & 0 & 0 & 27 & 4 & 0 & 1 & 32 & 32 \\
\hline ATFL & 0 & 0 & 0 & 0 & 0 & 0 & 0 & 0 & 0 & 0 & 0 & 0 & 1 & 2 & 2 & 0 & 0 & 5 & 5 \\
\hline WAVI & 0 & 0 & 0 & 0 & 0 & 0 & 0 & 0 & 0 & 0 & 0 & 0 & 0 & 15 & 1 & 0 & 0 & 16 & 16 \\
\hline WESJ & 0 & 0 & 0 & 0 & 0 & 0 & 0 & 0 & 0 & 0 & 0 & 0 & 0 & 2 & 0 & 0 & 0 & 2 & 2 \\
\hline BUSH & 9 & 7 & 1 & 7 & 0 & 24 & 0 & 5 & 0 & 0 & 0 & 5 & 18 & 0 & 0 & 0 & 0 & 18 & 47 \\
\hline BEWR & 0 & 0 & 0 & 1 & 0 & 1 & 0 & 0 & 0 & 0 & 0 & 0 & 6 & 2 & 3 & 1 & 1 & 13 & 14 \\
\hline SWTH & 0 & 0 & 0 & 0 & 0 & 0 & 0 & 0 & 0 & 0 & 0 & 0 & 0 & 3 & 1 & 0 & 0 & 4 & 4 \\
\hline WREN & 0 & 0 & 0 & 0 & 0 & 0 & 0 & 0 & 0 & 0 & 0 & 0 & 1 & 5 & 0 & 1 & 1 & 8 & 8 \\
\hline CATH & 0 & 0 & 0 & 0 & 0 & 0 & 0 & 0 & 0 & 0 & 0 & 0 & 2 & 2 & 0 & 1 & 0 & 5 & 5 \\
\hline OCWA & 0 & 2 & 5 & 2 & 0 & 9 & 0 & 0 & 5 & 8 & 0 & 13 & 3 & 0 & 0 & 1 & 0 & 4 & 26 \\
\hline AUWA & 0 & 0 & 0 & 0 & 0 & 0 & 0 & 0 & 1 & 0 & 0 & 1 & 0 & 0 & 0 & 0 & 0 & 0 & 1 \\
\hline TOWA & 0 & 0 & 2 & 2 & 0 & 4 & 0 & 0 & 2 & 0 & 0 & 2 & 0 & 1 & 0 & 0 & 0 & 1 & 7 \\
\hline HEWA & 0 & 0 & 1 & 0 & 0 & 1 & 0 & 0 & 2 & 1 & 0 & 3 & 0 & 0 & 0 & 0 & 0 & 0 & 4 \\
\hline COYE & 0 & 0 & 0 & 0 & 0 & 0 & 1 & 0 & 0 & 0 & 0 & 1 & 0 & 0 & 0 & 0 & 0 & 0 & 1 \\
\hline WIWA & 0 & 6 & 11 & 10 & 0 & 27 & 0 & 1 & 5 & 9 & 0 & 15 & 0 & 1 & 0 & 1 & 0 & 2 & 44 \\
\hline WETA & 0 & 0 & 0 & 0 & 0 & 0 & 0 & 0 & 1 & 1 & 0 & 2 & 0 & 0 & 0 & 0 & 0 & 0 & 2 \\
\hline SPTO & 0 & 4 & 1 & 2 & 0 & 7 & 0 & 0 & 1 & 2 & 0 & 3 & 2 & 0 & 0 & 0 & 0 & 2 & 12 \\
\hline
\end{tabular}




\begin{tabular}{|c|c|c|c|c|c|c|c|c|c|c|c|c|c|c|c|c|c|c|c|}
\hline \multirow{3}{*}{$\begin{array}{l}\text { Species } \\
\text { code }\end{array}$} & \multirow{2}{*}{\multicolumn{5}{|c|}{$\begin{array}{c}\text { Female } \\
\text { Age }\end{array}$}} & \multirow[b]{3}{*}{ Female total } & \multirow{2}{*}{\multicolumn{5}{|c|}{$\begin{array}{l}\text { Male } \\
\text { Age }\end{array}$}} & \multirow[b]{3}{*}{ Male total } & \multirow{2}{*}{\multicolumn{5}{|c|}{$\begin{array}{c}\text { Unknown sex } \\
\text { Age }\end{array}$}} & \multirow{3}{*}{$\begin{array}{c}\text { Unknown } \\
\text { total }\end{array}$} & \multirow{3}{*}{$\begin{array}{c}\text { Species } \\
\text { total }\end{array}$} \\
\hline & & & & & & & & & & & & & & & & & & & \\
\hline & HY & AHY & SY & ASY & I & & HY & AHY & SY & ASY & I & & HY & AHY & SY & ASY & I & & \\
\hline CALT & 0 & 3 & 0 & 0 & 0 & 3 & 0 & 0 & 1 & 0 & 0 & 1 & 2 & 2 & 1 & 0 & 0 & 5 & 9 \\
\hline SOSP & 0 & 1 & 0 & 0 & 0 & 1 & 0 & 1 & 0 & 0 & 0 & 1 & 1 & 1 & 0 & 0 & 0 & 2 & 4 \\
\hline$B L G R$ & 0 & 0 & 1 & 0 & 0 & 1 & 0 & 0 & 0 & 0 & 0 & 0 & 0 & 0 & 0 & 0 & 0 & 0 & 1 \\
\hline LAZB & 0 & 0 & 0 & 0 & 0 & 0 & 0 & 0 & 1 & 1 & 0 & 2 & 0 & 0 & 0 & 0 & 0 & 0 & 2 \\
\hline INBU & 0 & 0 & 0 & 0 & 0 & 0 & 0 & 0 & 0 & 0 & 0 & 0 & 0 & 0 & 1 & 0 & 0 & 1 & 1 \\
\hline HOOR & 0 & 0 & 0 & 1 & 0 & 1 & 0 & 0 & 2 & 0 & 0 & 2 & 6 & 0 & 0 & 0 & 1 & 7 & 10 \\
\hline $\mathrm{HOFI}$ & 0 & 1 & 0 & 0 & 0 & 1 & 0 & 0 & 2 & 0 & 0 & 2 & 0 & 0 & 0 & 0 & 0 & 0 & 3 \\
\hline Total & 18 & 38 & 22 & 25 & 2 & 105 & 18 & 14 & 23 & 22 & 3 & 80 & 43 & 72 & 14 & 5 & 7 & 141 & 326 \\
\hline
\end{tabular}


Table C20. Sex and age of individual birds (banded and unbanded) captured during Monitoring Avian Productivity and Survivorship (MAPS), Point Loma banding station, San Diego, California, 2013.

[See appendix A for bird species codes. Species in italics are non-breeding neotropical migrants. Age: HY=hatching-year, AHY=after-hatching-year, SY=second-year, ASY=after-secondyear, l=indeterminable age]

\begin{tabular}{|c|c|c|c|c|c|c|c|c|c|c|c|c|c|c|c|c|c|}
\hline \multirow{3}{*}{$\begin{array}{c}\text { Species } \\
\text { code }\end{array}$} & \multirow{2}{*}{\multicolumn{4}{|c|}{$\begin{array}{l}\text { Female } \\
\text { Age }\end{array}$}} & \multirow[b]{3}{*}{ Female total } & \multirow{2}{*}{\multicolumn{4}{|c|}{$\begin{array}{l}\text { Male } \\
\text { Age }\end{array}$}} & \multirow{3}{*}{$\begin{array}{l}\text { Male } \\
\text { total }\end{array}$} & \multicolumn{5}{|c|}{ Unknown sex } & \multirow{3}{*}{$\begin{array}{c}\text { Unknown } \\
\text { total }\end{array}$} & \multirow[b]{3}{*}{ Species total } \\
\hline & & & & & & & & & & & \multicolumn{5}{|c|}{ Age } & & \\
\hline & HY & AHY & SY & ASY & & HY & AHY & SY & ASY & & HY & AHY & SY & ASY & 1 & & \\
\hline ANHU & 15 & 2 & 0 & 0 & 17 & 24 & 2 & 0 & 0 & 26 & 2 & 0 & 0 & 0 & 1 & 3 & 46 \\
\hline $\mathrm{COHU}$ & 0 & 0 & 0 & 0 & 0 & 1 & 1 & 0 & 0 & 2 & 0 & 0 & 0 & 0 & 0 & 0 & 2 \\
\hline RUHU & 0 & 0 & 0 & 0 & 0 & 1 & 0 & 0 & 0 & 1 & 0 & 0 & 0 & 0 & 0 & 0 & 1 \\
\hline$A L H U$ & 0 & 4 & 0 & 0 & 4 & 2 & 1 & 0 & 0 & 3 & 0 & 0 & 0 & 0 & 0 & 0 & 7 \\
\hline USHU & 0 & 0 & 0 & 0 & 0 & 1 & 0 & 0 & 0 & 1 & 0 & 0 & 0 & 0 & 0 & 0 & 1 \\
\hline WEWP & 0 & 0 & 0 & 0 & 0 & 0 & 0 & 0 & 0 & 0 & 0 & 2 & 0 & 0 & 0 & 2 & 2 \\
\hline WIFL & 0 & 0 & 0 & 0 & 0 & 0 & 0 & 0 & 0 & 0 & 0 & 2 & 0 & 0 & 0 & 2 & 2 \\
\hline HAFL & 0 & 0 & 0 & 0 & 0 & 0 & 0 & 0 & 0 & 0 & 0 & 1 & 0 & 0 & 0 & 1 & 1 \\
\hline GRFL & 0 & 0 & 0 & 0 & 0 & 0 & 0 & 0 & 0 & 0 & 0 & 0 & 0 & 1 & 0 & 1 & 1 \\
\hline PSFL & 0 & 0 & 0 & 0 & 0 & 0 & 0 & 0 & 0 & 0 & 0 & 13 & 0 & 0 & 0 & 13 & 13 \\
\hline COFL & 0 & 0 & 0 & 0 & 0 & 0 & 0 & 0 & 0 & 0 & 0 & 1 & 0 & 0 & 0 & 1 & 1 \\
\hline ATFL & 0 & 0 & 0 & 0 & 0 & 0 & 0 & 0 & 0 & 0 & 0 & 1 & 1 & 1 & 0 & 3 & 3 \\
\hline LBVI & 0 & 0 & 0 & 0 & 0 & 0 & 0 & 0 & 0 & 0 & 2 & 0 & 0 & 0 & 0 & 2 & 2 \\
\hline CAVI & 0 & 0 & 0 & 0 & 0 & 0 & 0 & 0 & 0 & 0 & 0 & 1 & 0 & 0 & 0 & 1 & 1 \\
\hline WAVI & 0 & 0 & 0 & 0 & 0 & 0 & 0 & 0 & 0 & 0 & 0 & 3 & 3 & 1 & 0 & 7 & 7 \\
\hline REVI & 0 & 0 & 0 & 0 & 0 & 0 & 0 & 0 & 0 & 0 & 0 & 1 & 0 & 0 & 0 & 1 & 1 \\
\hline $\mathrm{BUSH}$ & 8 & 9 & 0 & 2 & 19 & 3 & 5 & 0 & 1 & 9 & 5 & 0 & 0 & 0 & 0 & 5 & 33 \\
\hline BEWR & 0 & 2 & 0 & 0 & 2 & 0 & 0 & 0 & 0 & 0 & 10 & 3 & 0 & 1 & 0 & 14 & 16 \\
\hline HOWR & 0 & 0 & 0 & 0 & 0 & 0 & 0 & 0 & 0 & 0 & 1 & 0 & 0 & 0 & 0 & 1 & 1 \\
\hline CAGN & 0 & 0 & 0 & 0 & 0 & 0 & 0 & 0 & 0 & 0 & 1 & 0 & 0 & 0 & 0 & 1 & 1 \\
\hline SWTH & 0 & 0 & 0 & 0 & 0 & 0 & 0 & 0 & 0 & 0 & 0 & 1 & 0 & 1 & 0 & 2 & 2 \\
\hline WREN & 0 & 0 & 0 & 0 & 0 & 0 & 0 & 0 & 0 & 0 & 4 & 4 & 0 & 1 & 0 & 9 & 9 \\
\hline NOMO & 0 & 0 & 0 & 1 & 1 & 0 & 0 & 0 & 0 & 0 & 0 & 0 & 0 & 0 & 0 & 0 & 1 \\
\hline CATH & 0 & 0 & 0 & 0 & 0 & 0 & 0 & 0 & 0 & 0 & 1 & 1 & 0 & 1 & 0 & 3 & 3 \\
\hline OCWA & 0 & 4 & 1 & 9 & 14 & 0 & 1 & 0 & 6 & 7 & 6 & 1 & 0 & 1 & 0 & 8 & 29 \\
\hline TOWA & 0 & 0 & 0 & 0 & 0 & 0 & 1 & 0 & 0 & 1 & 0 & 0 & 0 & 0 & 0 & 0 & 1 \\
\hline
\end{tabular}




\begin{tabular}{|c|c|c|c|c|c|c|c|c|c|c|c|c|c|c|c|c|c|}
\hline \multirow{3}{*}{$\begin{array}{l}\text { Species } \\
\text { code }\end{array}$} & \multirow{2}{*}{\multicolumn{4}{|c|}{$\begin{array}{c}\text { Female } \\
\text { Age }\end{array}$}} & \multirow[b]{3}{*}{ Female total } & \multirow{2}{*}{\multicolumn{4}{|c|}{$\begin{array}{l}\text { Male } \\
\text { Age } \\
\end{array}$}} & \multirow{3}{*}{$\begin{array}{l}\text { Male } \\
\text { total }\end{array}$} & \multirow{2}{*}{\multicolumn{5}{|c|}{$\begin{array}{c}\text { Unknown sex } \\
\text { Age }\end{array}$}} & \multirow{3}{*}{$\begin{array}{c}\text { Unknown } \\
\text { total }\end{array}$} & \multirow[b]{3}{*}{ Species total } \\
\hline & & & & & & & & & & & & & & & & & \\
\hline & HY & AHY & SY & ASY & & HY & AHY & SY & ASY & & HY & AHY & SY & ASY & 1 & & \\
\hline HEWA & 0 & 0 & 1 & 0 & 1 & 0 & 0 & 0 & 0 & 0 & 0 & 1 & 0 & 0 & 0 & 1 & 2 \\
\hline MGWA & 0 & 0 & 0 & 0 & 0 & 0 & 0 & 0 & 1 & 1 & 0 & 0 & 0 & 0 & 0 & 0 & 1 \\
\hline WIWA & 0 & 0 & 1 & 3 & 4 & 0 & 1 & 0 & 1 & 2 & 0 & 0 & 0 & 0 & 0 & 0 & 6 \\
\hline WETA & 0 & 0 & 1 & 2 & 3 & 0 & 0 & 0 & 0 & 0 & 0 & 0 & 0 & 0 & 0 & 0 & 3 \\
\hline SPTO & 0 & 3 & 2 & 0 & 5 & 0 & 1 & 2 & 7 & 10 & 11 & 0 & 0 & 0 & 0 & 11 & 26 \\
\hline CALT & 0 & 2 & 0 & 1 & 3 & 0 & 1 & 1 & 0 & 2 & 0 & 2 & 0 & 0 & 1 & 3 & 8 \\
\hline$B H G R$ & 0 & 0 & 1 & 1 & 2 & 0 & 0 & 0 & 0 & 0 & 0 & 0 & 0 & 0 & 0 & 0 & 2 \\
\hline HOOR & 0 & 1 & 0 & 0 & 1 & 0 & 1 & 3 & 0 & 4 & 3 & 0 & 0 & 0 & 0 & 3 & 8 \\
\hline $\mathrm{HOFI}$ & 0 & 3 & 0 & 0 & 3 & 0 & 3 & 0 & 0 & 3 & 5 & 0 & 0 & 0 & 0 & 5 & 11 \\
\hline LEGO & 0 & 1 & 0 & 0 & 1 & 0 & 0 & 0 & 0 & 0 & 1 & 0 & 0 & 0 & 0 & 1 & 2 \\
\hline Total & 23 & 31 & 7 & 19 & 80 & 32 & 18 & 6 & 16 & 72 & 52 & 38 & 4 & 8 & 2 & 104 & 256 \\
\hline
\end{tabular}


Table C21. Sex and age of individual birds (banded and unbanded) captured during Monitoring Avian Productivity and Survivorship (MAPS), Point Loma banding station, San Diego, California, 2014.

\begin{tabular}{|c|c|c|c|c|c|c|c|c|c|c|c|c|c|c|c|c|}
\hline \multirow{3}{*}{$\begin{array}{l}\text { Species } \\
\text { code }\end{array}$} & \multirow{2}{*}{\multicolumn{4}{|c|}{ Female }} & \multirow{3}{*}{$\begin{array}{c}\text { Female } \\
\text { total }\end{array}$} & \multirow{2}{*}{\multicolumn{4}{|c|}{ Male }} & \multirow{3}{*}{$\begin{array}{l}\text { Male } \\
\text { total }\end{array}$} & \multirow{2}{*}{\multicolumn{4}{|c|}{$\begin{array}{r}\text { Unknown sex } \\
\text { Age } \\
\end{array}$}} & \multirow{3}{*}{$\begin{array}{c}\text { Unknown } \\
\text { total }\end{array}$} & \multirow{3}{*}{$\begin{array}{c}\text { Species } \\
\text { total }\end{array}$} \\
\hline & & & & & & & & & & & & & & & & \\
\hline & HY & AHY & SY & ASY & & HY & AHY & SY & ASY & & HY & AHY & sY & ASY & & \\
\hline$B C H U$ & 1 & 0 & 0 & 0 & 0 & 0 & 0 & 0 & 0 & 0 & 0 & 0 & 0 & 0 & 0 & 1 \\
\hline ANHU & 12 & 7 & 0 & 0 & 3 & 23 & 3 & 7 & 0 & 33 & 2 & 1 & 1 & 0 & 4 & 56 \\
\hline $\mathrm{COHU}$ & 0 & 0 & 0 & 0 & 2 & 6 & 2 & 0 & 0 & 8 & 0 & 0 & 0 & 0 & 0 & 8 \\
\hline $\mathrm{CAHU}$ & 0 & 2 & 0 & 0 & 0 & 0 & 0 & 0 & 0 & 0 & 0 & 0 & 0 & 0 & 0 & 2 \\
\hline$A L H U$ & 1 & 2 & 0 & 0 & 3 & 4 & 3 & 1 & 0 & 8 & 0 & 0 & 0 & 0 & 0 & 11 \\
\hline USHU & 0 & 0 & 0 & 0 & 1 & 0 & 1 & 0 & 0 & 1 & 0 & 0 & 0 & 0 & 0 & 1 \\
\hline DOWO & 0 & 1 & 0 & 0 & 0 & 0 & 0 & 0 & 0 & 0 & 0 & 0 & 0 & 0 & 0 & 1 \\
\hline WEWP & 0 & 0 & 0 & 0 & 0 & 0 & 0 & 0 & 0 & 0 & 0 & 1 & 0 & 0 & 1 & 1 \\
\hline WIFL & 0 & 0 & 0 & 0 & 0 & 0 & 0 & 0 & 0 & 0 & 0 & 1 & 1 & 0 & 2 & 2 \\
\hline HAFL & 0 & 0 & 0 & 0 & 0 & 0 & 0 & 0 & 0 & 0 & 0 & 2 & 0 & 0 & 2 & 2 \\
\hline$P S F L$ & 0 & 1 & 0 & 0 & 0 & 0 & 0 & 0 & 0 & 0 & 1 & 70 & 2 & 0 & 73 & 74 \\
\hline ATFL & 0 & 0 & 0 & 0 & 0 & 0 & 0 & 0 & 0 & 0 & 0 & 0 & 3 & 0 & 3 & 3 \\
\hline LBVI & 0 & 0 & 0 & 0 & 0 & 0 & 0 & 0 & 0 & 0 & 0 & 0 & 1 & 0 & 1 & 1 \\
\hline WAVI & 0 & 0 & 0 & 0 & 0 & 0 & 0 & 0 & 0 & 0 & 0 & 13 & 17 & 1 & 31 & 31 \\
\hline $\mathrm{BUSH}$ & 0 & 5 & 1 & 0 & 4 & 0 & 4 & 0 & 0 & 4 & 0 & 0 & 0 & 0 & 0 & 10 \\
\hline BEWR & 0 & 1 & 0 & 4 & 0 & 0 & 0 & 0 & 0 & 0 & 1 & 3 & 0 & 1 & 5 & 10 \\
\hline SWTH & 0 & 0 & 0 & 0 & 0 & 0 & 0 & 0 & 0 & 0 & 0 & 7 & 1 & 0 & 8 & 8 \\
\hline HETH & 0 & 0 & 0 & 0 & 0 & 0 & 0 & 0 & 0 & 0 & 0 & 1 & 0 & 0 & 1 & 1 \\
\hline WREN & 0 & 0 & 0 & 0 & 0 & 0 & 0 & 0 & 0 & 0 & 1 & 7 & 2 & 4 & 14 & 14 \\
\hline NOMO & 0 & 0 & 0 & 0 & 0 & 0 & 0 & 0 & 0 & 0 & 0 & 0 & 0 & 1 & 1 & 1 \\
\hline CATH & 0 & 0 & 0 & 0 & 0 & 0 & 0 & 0 & 0 & 0 & 1 & 0 & 0 & 1 & 2 & 2 \\
\hline OCWA & 0 & 1 & 1 & 1 & 1 & 0 & 1 & 1 & 3 & 5 & 2 & 2 & 0 & 0 & 4 & 12 \\
\hline YEWA & 0 & 0 & 1 & 0 & 1 & 0 & 1 & 1 & 0 & 2 & 0 & 0 & 2 & 0 & 2 & 5 \\
\hline TOWA & 0 & 0 & 6 & 0 & 0 & 0 & 0 & 2 & 0 & 2 & 0 & 0 & 0 & 0 & 0 & 8 \\
\hline HEWA & 0 & 0 & 3 & 0 & 0 & 0 & 0 & 0 & 0 & 0 & 0 & 0 & 0 & 0 & 0 & 3 \\
\hline MGWA & 0 & 1 & 1 & 1 & 2 & 0 & 2 & 1 & 1 & 4 & 0 & 0 & 0 & 0 & 0 & 7 \\
\hline WIWA & 0 & 7 & 10 & 5 & 0 & 0 & 0 & 1 & 1 & 2 & 0 & 3 & 0 & 0 & 3 & 27 \\
\hline WETA & 0 & 0 & 0 & 0 & 0 & 0 & 0 & 2 & 0 & 2 & 0 & 1 & 0 & 0 & 1 & 3 \\
\hline SPTO & 0 & 0 & 2 & 1 & 1 & 0 & 1 & 1 & 5 & 7 & 2 & 0 & 0 & 0 & 2 & 12 \\
\hline CALT & 0 & 1 & 0 & 2 & 0 & 0 & 0 & 0 & 2 & 2 & 2 & 2 & 0 & 1 & 5 & 10 \\
\hline SAGS & 0 & 0 & 0 & 0 & 0 & 0 & 0 & 0 & 0 & 0 & 0 & 1 & 0 & 0 & 1 & 1 \\
\hline ORJU & 0 & 0 & 0 & 0 & 0 & 0 & 0 & 0 & 0 & 0 & 1 & 0 & 0 & 0 & 1 & 1 \\
\hline HOOR & 0 & 1 & 0 & 1 & 0 & 0 & 0 & 0 & 1 & 1 & 2 & 0 & 0 & 0 & 2 & 5 \\
\hline $\mathrm{HOFI}$ & 0 & 0 & 0 & 0 & 1 & 0 & 1 & 0 & 0 & 1 & 3 & 0 & 0 & 0 & 3 & 4 \\
\hline Total & 14 & 30 & 25 & 15 & 33 & 33 & 19 & 17 & 13 & 82 & 18 & 115 & 30 & 9 & 172 & 338 \\
\hline
\end{tabular}


Table C22. Sex and age of individual birds (banded and unbanded) captured during Monitoring Avian Productivity and Survivorship (MAPS), Point Loma banding station, San Diego, California, 2015.

[See appendix A for bird species codes. Species in italics are non-breeding neotropical migrants. Age: $H Y=$ hatching-year, $A H Y=a f t e r-$ hatching-year, SY=second-year, ASY=after-second-year, l=indeterminable age]

\begin{tabular}{|c|c|c|c|c|c|c|c|c|c|c|c|c|c|c|c|c|c|c|}
\hline \multirow{3}{*}{$\begin{array}{l}\text { Species } \\
\text { code }\end{array}$} & \multirow{2}{*}{\multicolumn{5}{|c|}{$\begin{array}{c}\text { Female } \\
\text { Age }\end{array}$}} & \multirow{3}{*}{$\begin{array}{c}\begin{array}{c}\text { Female } \\
\text { total }\end{array} \\
\end{array}$} & \multirow{2}{*}{\multicolumn{4}{|c|}{$\begin{array}{l}\text { Male } \\
\text { Age }\end{array}$}} & \multirow{3}{*}{$\begin{array}{l}\text { Male } \\
\text { total }\end{array}$} & \multicolumn{5}{|c|}{ Unknown sex } & \multirow{3}{*}{$\begin{array}{c}\text { Unknown } \\
\text { total }\end{array}$} & \multirow{3}{*}{$\begin{array}{c}\text { Species } \\
\text { total }\end{array}$} \\
\hline & & & & & & & & & & & & \multicolumn{5}{|c|}{ Age } & & \\
\hline & $\mathrm{HY}$ & $\mathrm{AHY}$ & SY & ASY & 1 & & $\mathrm{HY}$ & AHY & SY & ASY & & HY & AHY & sY & ASY & 1 & & \\
\hline AMKE & 0 & 0 & 0 & 0 & 0 & 0 & 0 & 1 & 0 & 0 & 1 & 0 & 0 & 0 & 0 & 0 & 0 & 1 \\
\hline MODO & 0 & 0 & 0 & 0 & 0 & 0 & 0 & 1 & 0 & 0 & 1 & 0 & 0 & 0 & 0 & 0 & 0 & 1 \\
\hline ANHU & 1 & 1 & 0 & 0 & 0 & 2 & 3 & 3 & 0 & 0 & 6 & 0 & 0 & 0 & 0 & 0 & 0 & 8 \\
\hline $\mathrm{COHU}$ & 0 & 1 & 0 & 0 & 0 & 1 & 1 & 1 & 0 & 0 & 2 & 0 & 0 & 0 & 0 & 0 & 0 & 3 \\
\hline CAHU & 0 & 2 & 0 & 0 & 0 & 2 & 0 & 0 & 0 & 0 & 0 & 0 & 0 & 0 & 0 & 0 & 0 & 2 \\
\hline ALHU & 2 & 1 & 0 & 0 & 0 & 3 & 0 & 0 & 0 & 0 & 0 & 0 & 0 & 0 & 0 & 0 & 0 & 3 \\
\hline WEWP & 0 & 0 & 0 & 0 & 0 & 0 & 0 & 0 & 0 & 0 & 0 & 0 & 5 & 0 & 0 & 0 & 5 & 5 \\
\hline WIFL & 0 & 0 & 0 & 0 & 0 & 0 & 0 & 0 & 0 & 0 & 0 & 0 & 3 & 0 & 0 & 0 & 3 & 3 \\
\hline HAFL & 0 & 0 & 0 & 0 & 0 & 0 & 0 & 0 & 0 & 0 & 0 & 0 & 1 & 0 & 0 & 0 & 1 & 1 \\
\hline$P S F L$ & 0 & 0 & 0 & 1 & 0 & 1 & 0 & 0 & 0 & 0 & 0 & 0 & 29 & 1 & 0 & 0 & 30 & 31 \\
\hline ATFL & 0 & 0 & 0 & 0 & 0 & 0 & 0 & 0 & 0 & 0 & 0 & 0 & 1 & 1 & 0 & 0 & 2 & 2 \\
\hline CAKI & 0 & 0 & 0 & 0 & 0 & 0 & 0 & 1 & 0 & 0 & 1 & 0 & 0 & 0 & 0 & 0 & 0 & 1 \\
\hline WAVI & 0 & 0 & 0 & 0 & 0 & 0 & 0 & 0 & 0 & 0 & 0 & 0 & 18 & 4 & 3 & 0 & 25 & 25 \\
\hline BARS & 0 & 0 & 0 & 0 & 0 & 0 & 0 & 0 & 0 & 0 & 0 & 1 & 0 & 0 & 0 & 0 & 1 & 1 \\
\hline $\mathrm{BUSH}$ & 3 & 0 & 0 & 2 & 2 & 7 & 0 & 2 & 0 & 2 & 4 & 7 & 0 & 0 & 0 & 0 & 7 & 18 \\
\hline BEWR & 0 & 2 & 0 & 2 & 0 & 4 & 0 & 0 & 0 & 1 & 1 & 21 & 4 & 0 & 0 & 1 & 26 & 31 \\
\hline RCKI & 0 & 1 & 0 & 0 & 0 & 1 & 0 & 0 & 0 & 0 & 0 & 0 & 0 & 0 & 0 & 0 & 0 & 1 \\
\hline CAGN & 0 & 0 & 0 & 0 & 0 & 0 & 0 & 0 & 0 & 0 & 0 & 1 & 0 & 0 & 0 & 0 & 1 & 1 \\
\hline WREN & 0 & 0 & 0 & 0 & 0 & 0 & 0 & 0 & 0 & 0 & 0 & 15 & 6 & 0 & 2 & 2 & 25 & 25 \\
\hline GRCA & 0 & 0 & 0 & 0 & 0 & 0 & 0 & 0 & 0 & 0 & 0 & 0 & 0 & 0 & 1 & 0 & 1 & 1 \\
\hline NOMO & 0 & 0 & 0 & 0 & 0 & 0 & 0 & 0 & 0 & 0 & 0 & 1 & 0 & 0 & 0 & 0 & 1 & 1 \\
\hline CATH & 0 & 0 & 0 & 0 & 0 & 0 & 0 & 0 & 0 & 1 & 1 & 0 & 3 & 0 & 0 & 3 & 6 & 7 \\
\hline OCWA & 0 & 2 & 0 & 3 & 0 & 5 & 0 & 1 & 0 & 3 & 4 & 7 & 0 & 1 & 0 & 1 & 9 & 18 \\
\hline YEWA & 0 & 2 & 1 & 0 & 0 & 3 & 0 & 0 & 1 & 0 & 1 & 0 & 1 & 0 & 0 & 0 & 1 & 5 \\
\hline TOWA & 0 & 1 & 3 & 0 & 0 & 4 & 0 & 0 & 1 & 0 & 1 & 0 & 0 & 0 & 0 & 0 & 0 & 5 \\
\hline HEWA & 0 & 0 & 0 & 0 & 0 & 0 & 0 & 0 & 1 & 0 & 1 & 0 & 0 & 0 & 0 & 0 & 0 & 1 \\
\hline WIWA & 0 & 5 & 9 & 6 & 0 & 20 & 0 & 3 & 4 & 4 & 11 & 0 & 0 & 0 & 0 & 0 & 0 & 31 \\
\hline CAWA & 0 & 1 & 0 & 0 & 0 & 1 & 0 & 0 & 0 & 0 & 0 & 0 & 0 & 0 & 0 & 0 & 0 & 1 \\
\hline WETA & 0 & 0 & 1 & 2 & 0 & 3 & 0 & 0 & 0 & 0 & 0 & 0 & 0 & 0 & 0 & 0 & 0 & 3 \\
\hline SPTO & 0 & 2 & 0 & 1 & 0 & 3 & 0 & 0 & 2 & 4 & 6 & 16 & 0 & 0 & 0 & 0 & 16 & 25 \\
\hline CALT & 0 & 1 & 0 & 2 & 0 & 3 & 0 & 1 & 0 & 2 & 3 & 13 & 0 & 0 & 0 & 1 & 14 & 20 \\
\hline LAZB & 0 & 0 & 0 & 0 & 0 & 0 & 0 & 0 & 0 & 0 & 0 & 1 & 0 & 0 & 0 & 0 & 1 & 1 \\
\hline HOOR & 0 & 0 & 0 & 2 & 0 & 2 & 0 & 0 & 0 & 1 & 1 & 0 & 0 & 0 & 0 & 0 & 0 & 3 \\
\hline HOFI & 1 & 0 & 0 & 0 & 0 & 1 & 0 & 0 & 0 & 0 & 0 & 0 & 0 & 0 & 0 & 0 & 0 & 1 \\
\hline LEGO & 3 & 0 & 0 & 0 & 0 & 3 & 3 & 0 & 0 & 0 & 3 & 0 & 0 & 0 & 0 & 0 & 0 & 6 \\
\hline Total & 10 & 22 & 14 & 21 & 2 & 69 & 7 & 14 & 9 & 18 & 48 & 83 & 71 & 7 & 6 & 8 & 175 & 293 \\
\hline
\end{tabular}




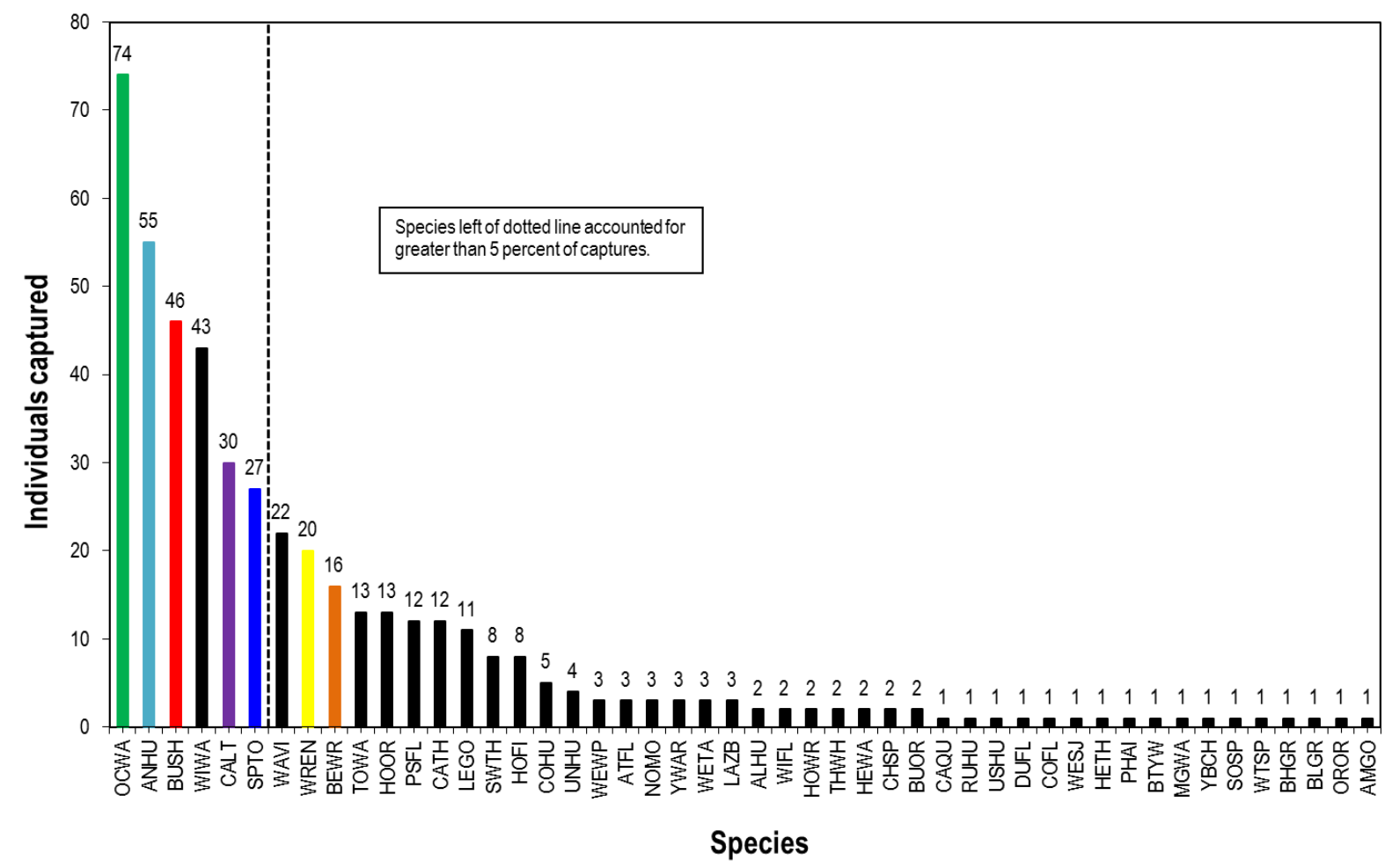

Figure C1. Number of individuals captured per species, Point Loma MAPS banding station, 2011. Colored bars represent the seven most commonly captured resident species from 2011 to 2015.

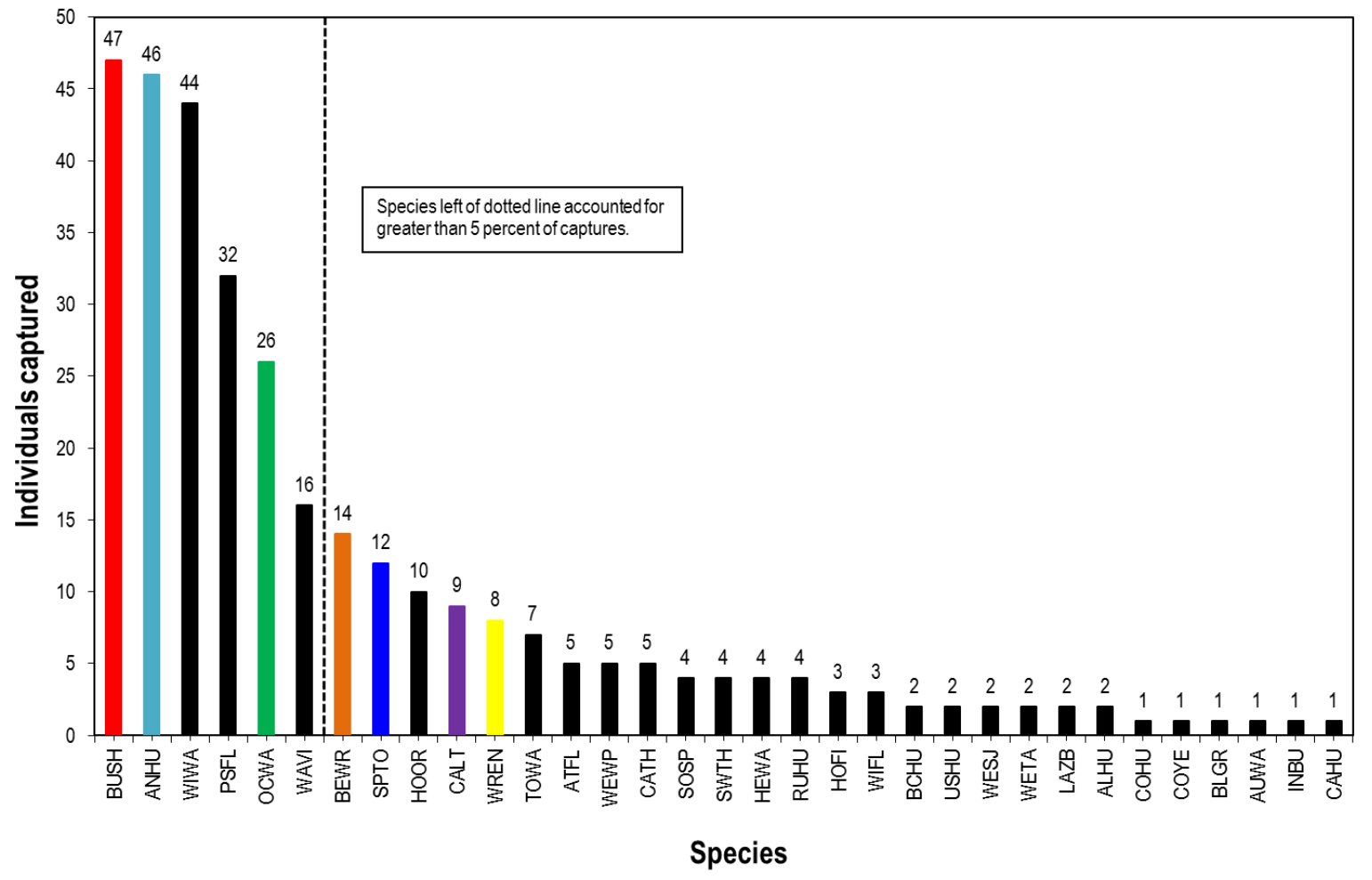

Figure C2. Number of individuals captured per species, Point Loma MAPS banding station, 2012. Colored bars represent the seven most commonly captured resident species from 2011 to 2015. 


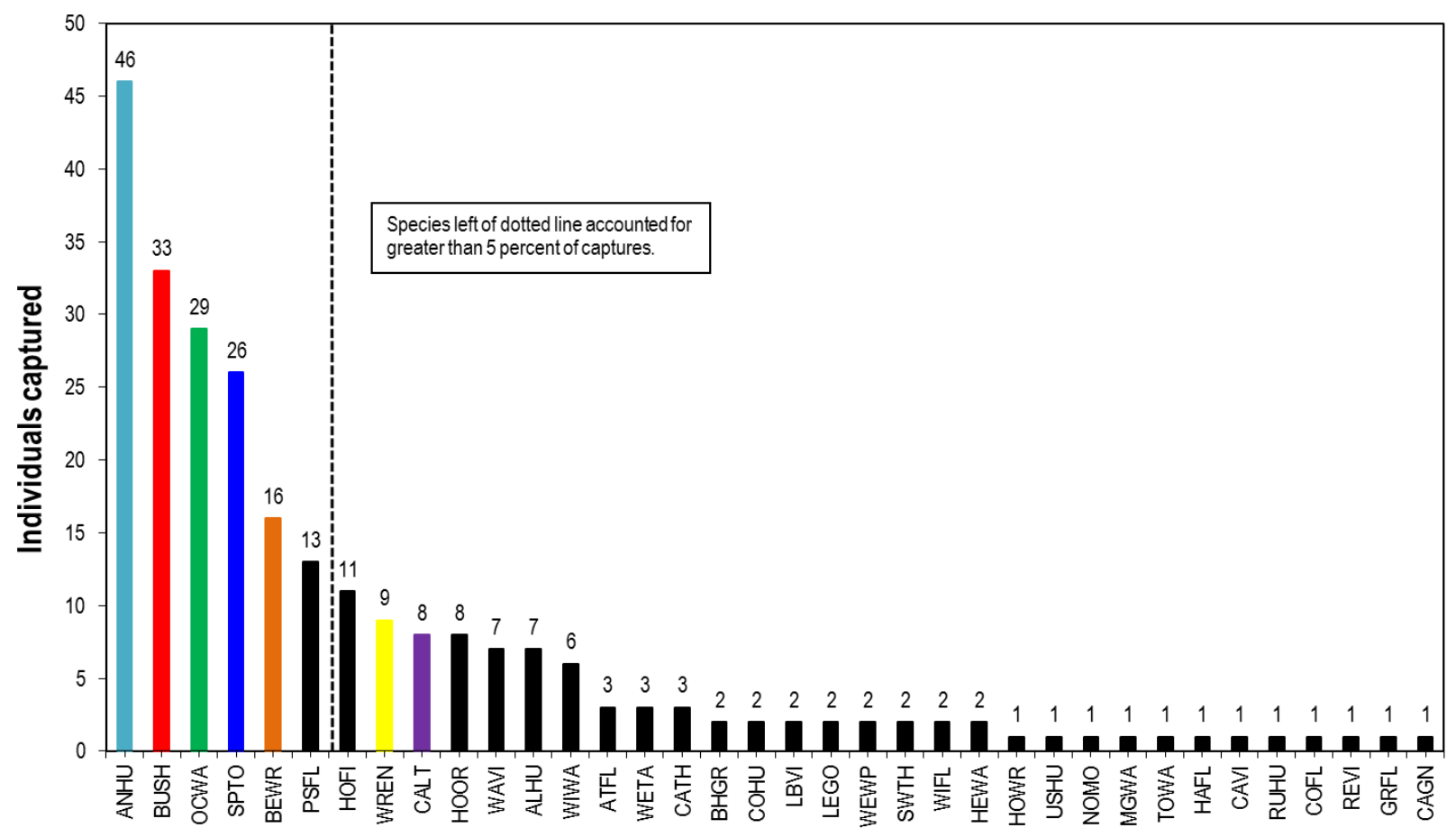

Species

Figure C3. Number of individuals captured per species, Point Loma MAPS banding station, 2013. Colored bars represent the seven most commonly captured resident species from 2011 to 2015.

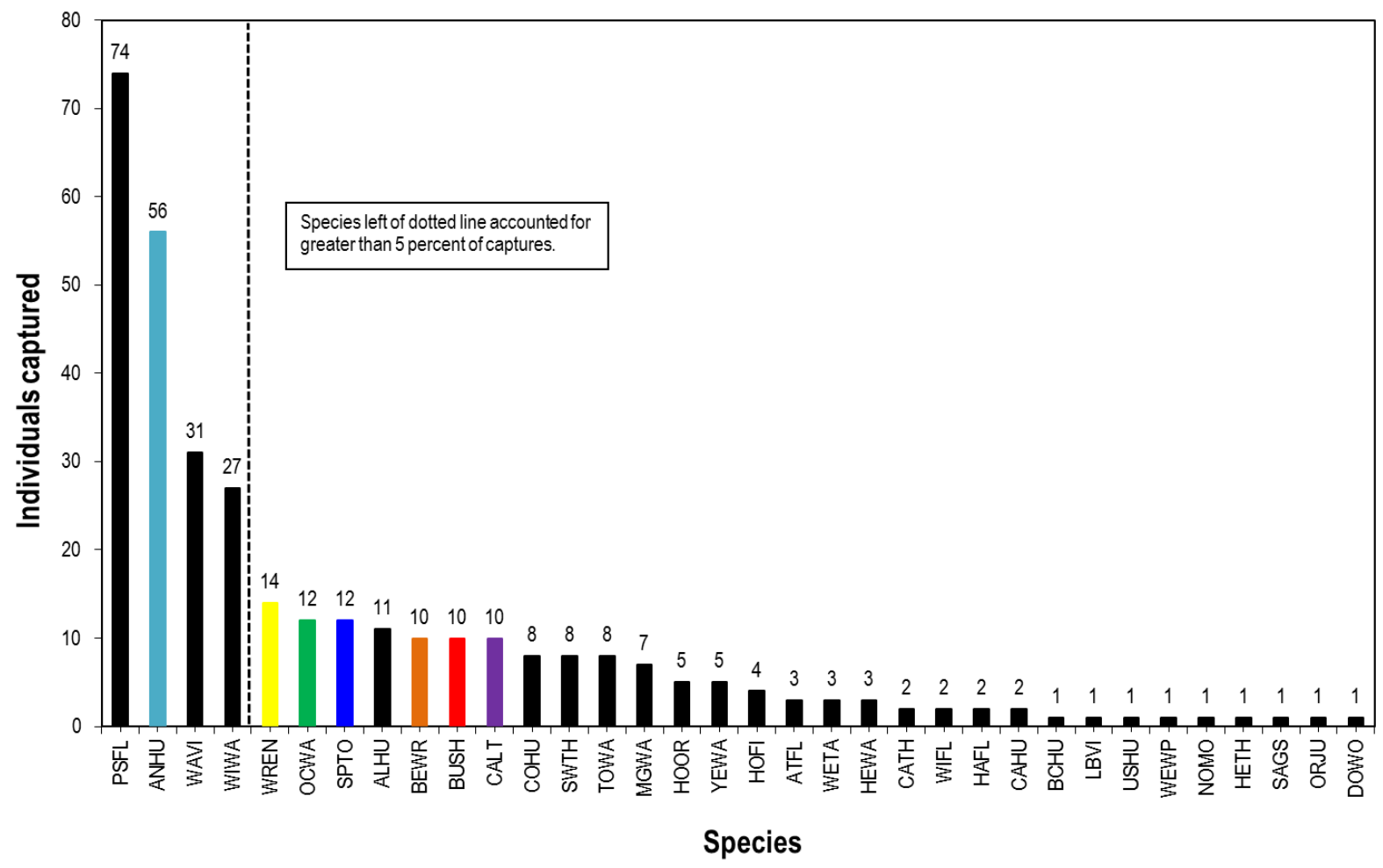

Figure C4. Number of individuals captured per species, Point Loma MAPS banding station, 2014. Colored bars represent the seven most commonly captured resident species from 2011 to 2015. 


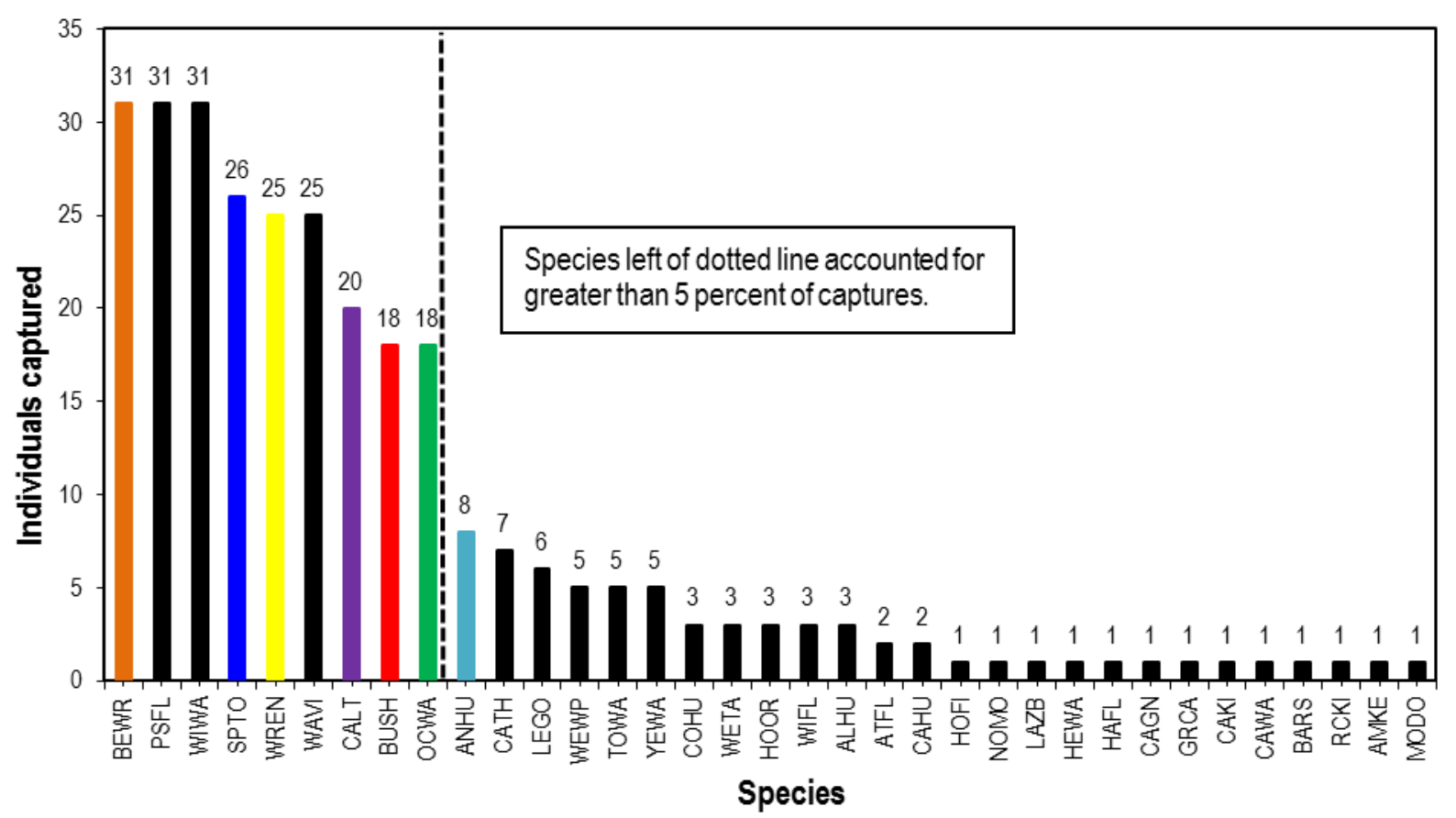

Figure C5. Number of individuals captured per species, Point Loma MAPS banding station, 2015. Colored bars represent the seven most commonly captured resident species from 2011 to 2015.

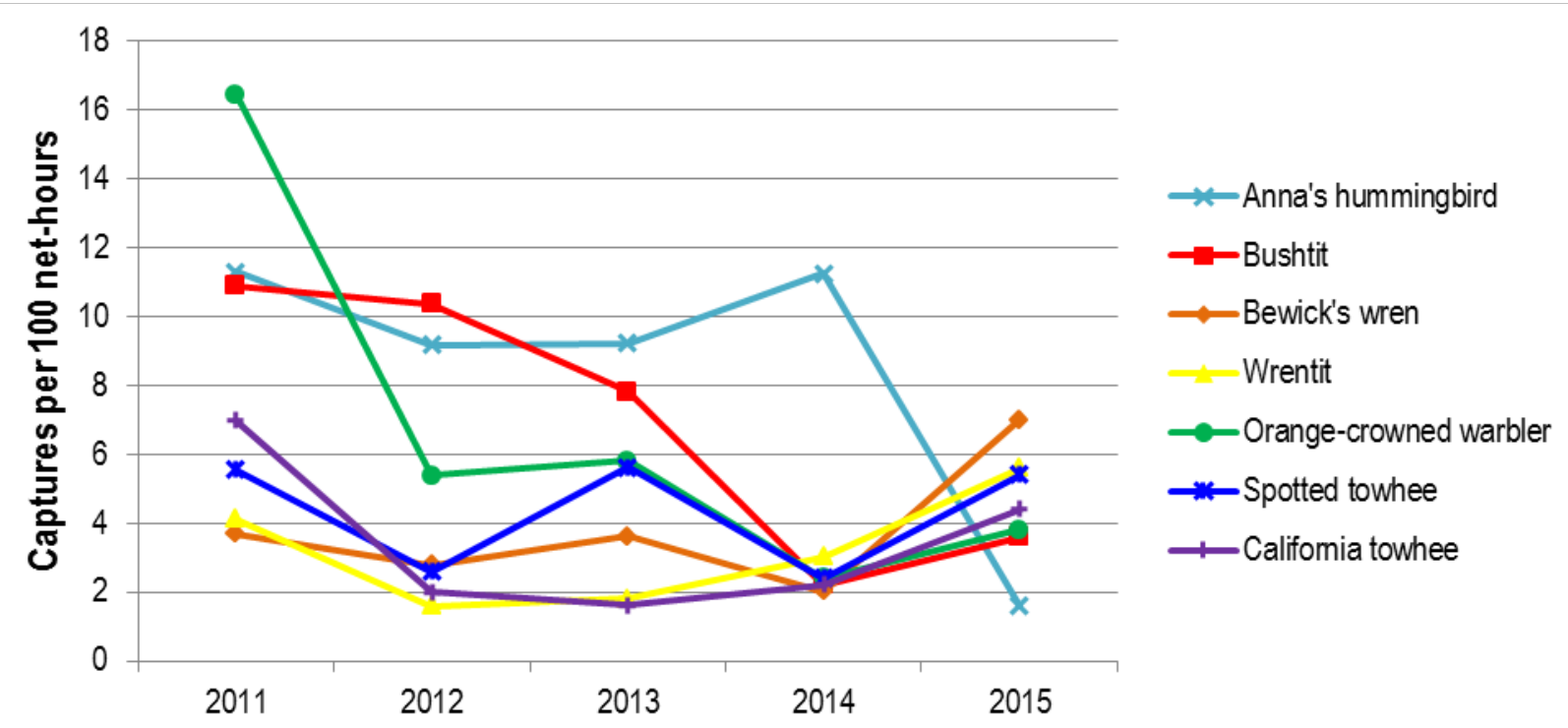

Figure $\mathbf{C 6}$. Annual capture rate (captures per 100 net-hours) of the seven most commonly captured resident species at Point Loma MAPS banding station, 2011-15. 


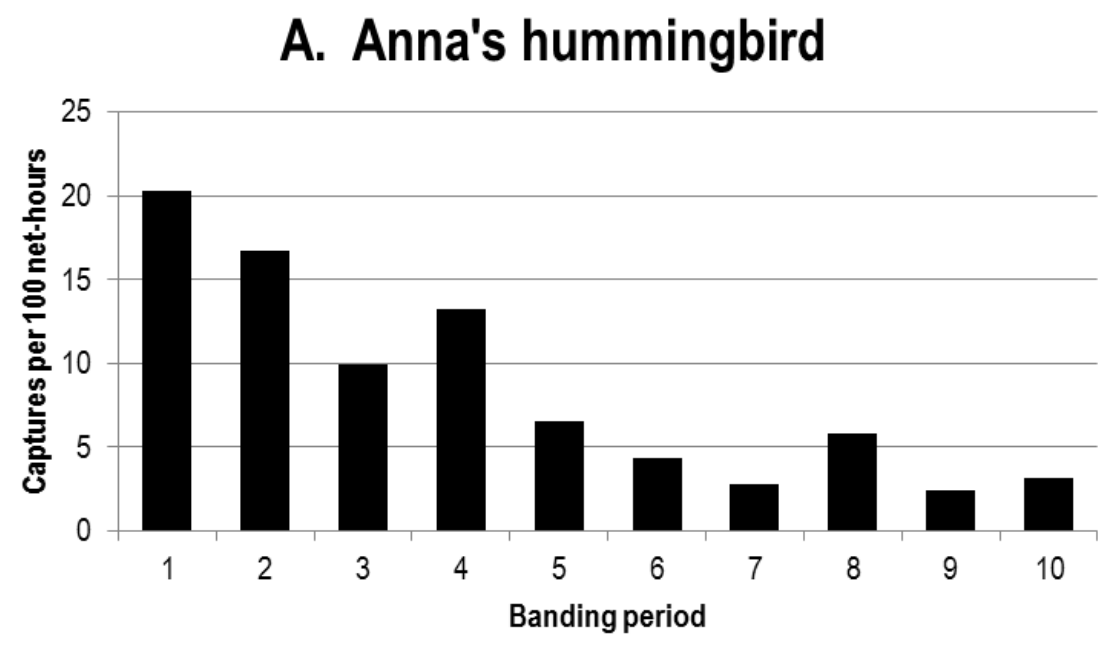

B. Bushtit

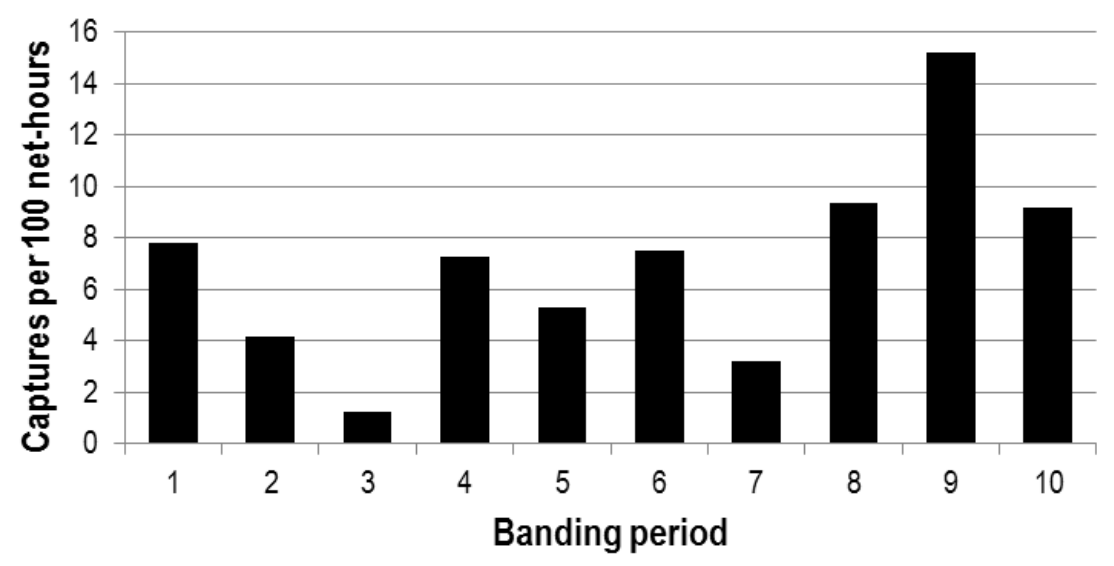

\section{Bewick's wren}

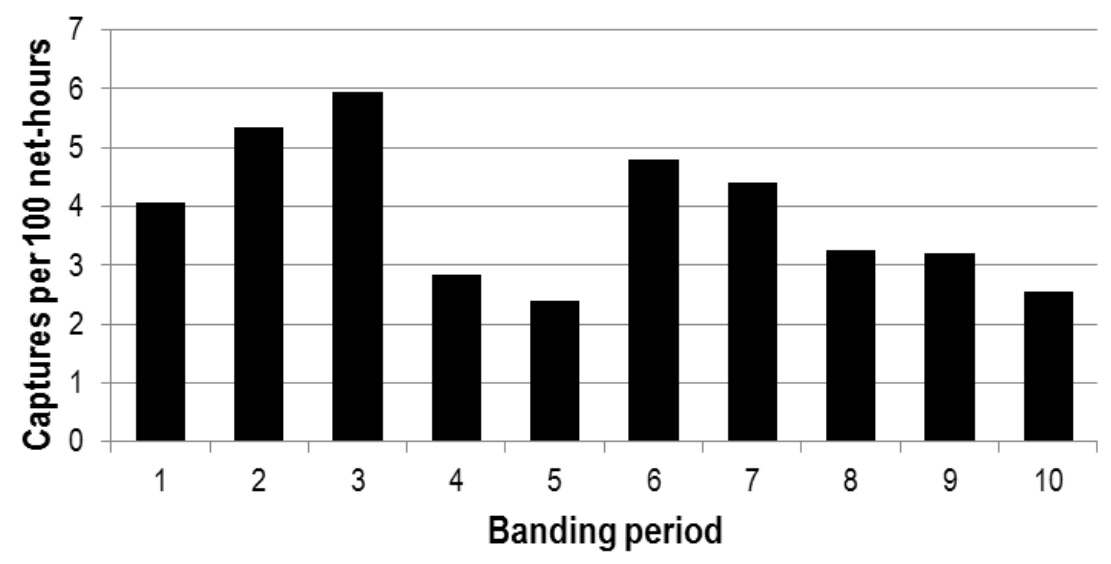

Figure C7. Average seasonal capture rate (captures per 100 net-hours) of the seven most commonly captured species at Point Loma MAPS banding station, 2011-15. Banding periods were: 1=1-10 May; 2=11-20 May; 3=21-30 May; 4= 31 May-9 June; 5=10-19 June; 6=20-29 June; 7=30 June-9 July; 8=10-19 July; 9=2029 July; $10=30$ July-8 August. 


\section{Wrentit}

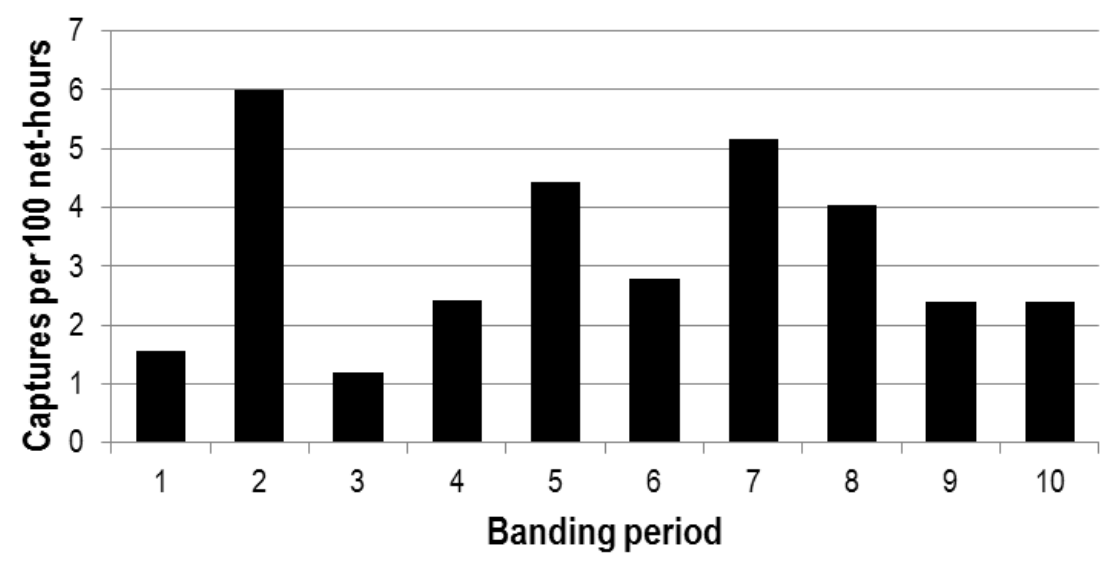

E. Orange-crowned warbler

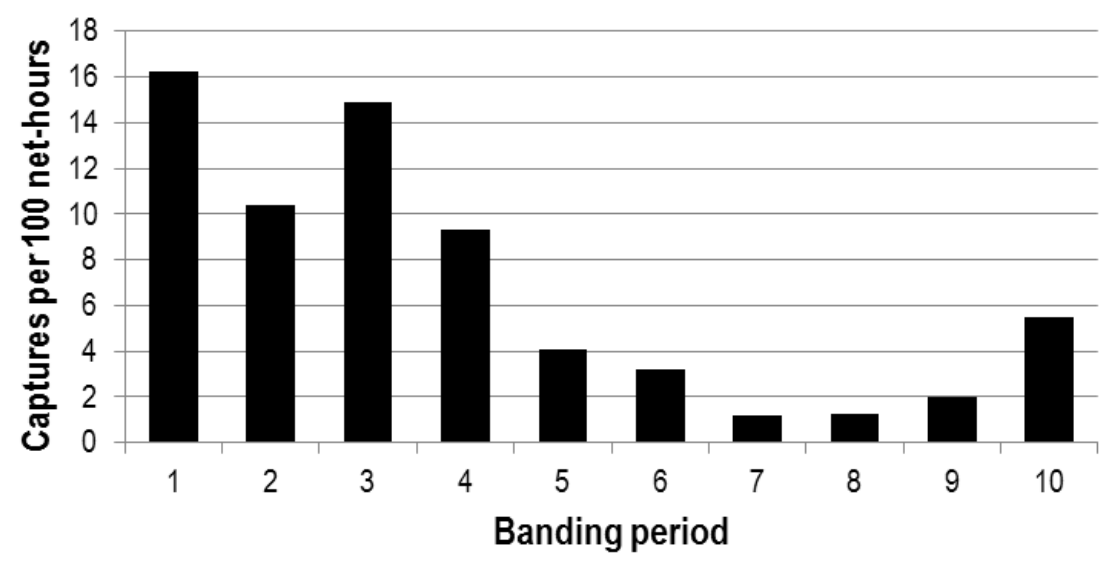

F. Spotted towhee

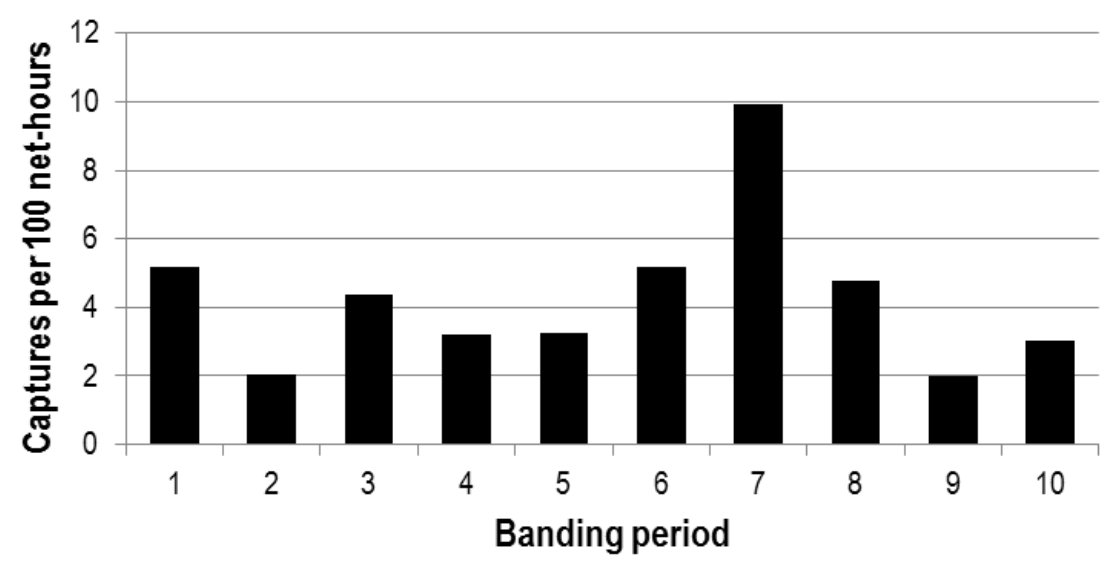

Figure C7.-Continued. 


\section{G. California towhee}

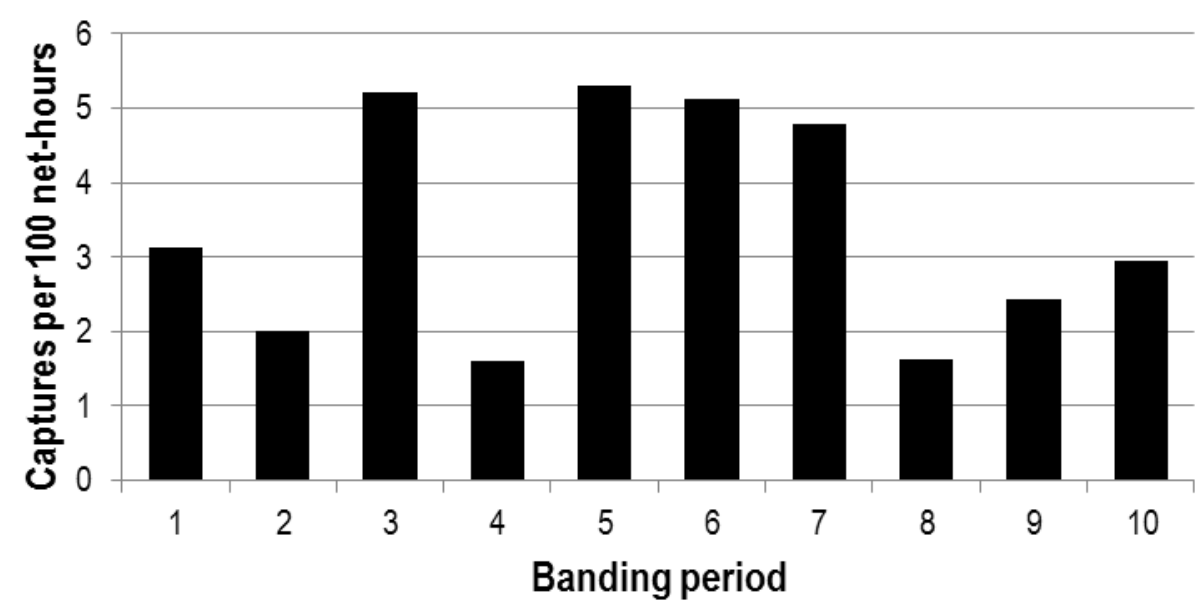

Figure C7.-Continued.

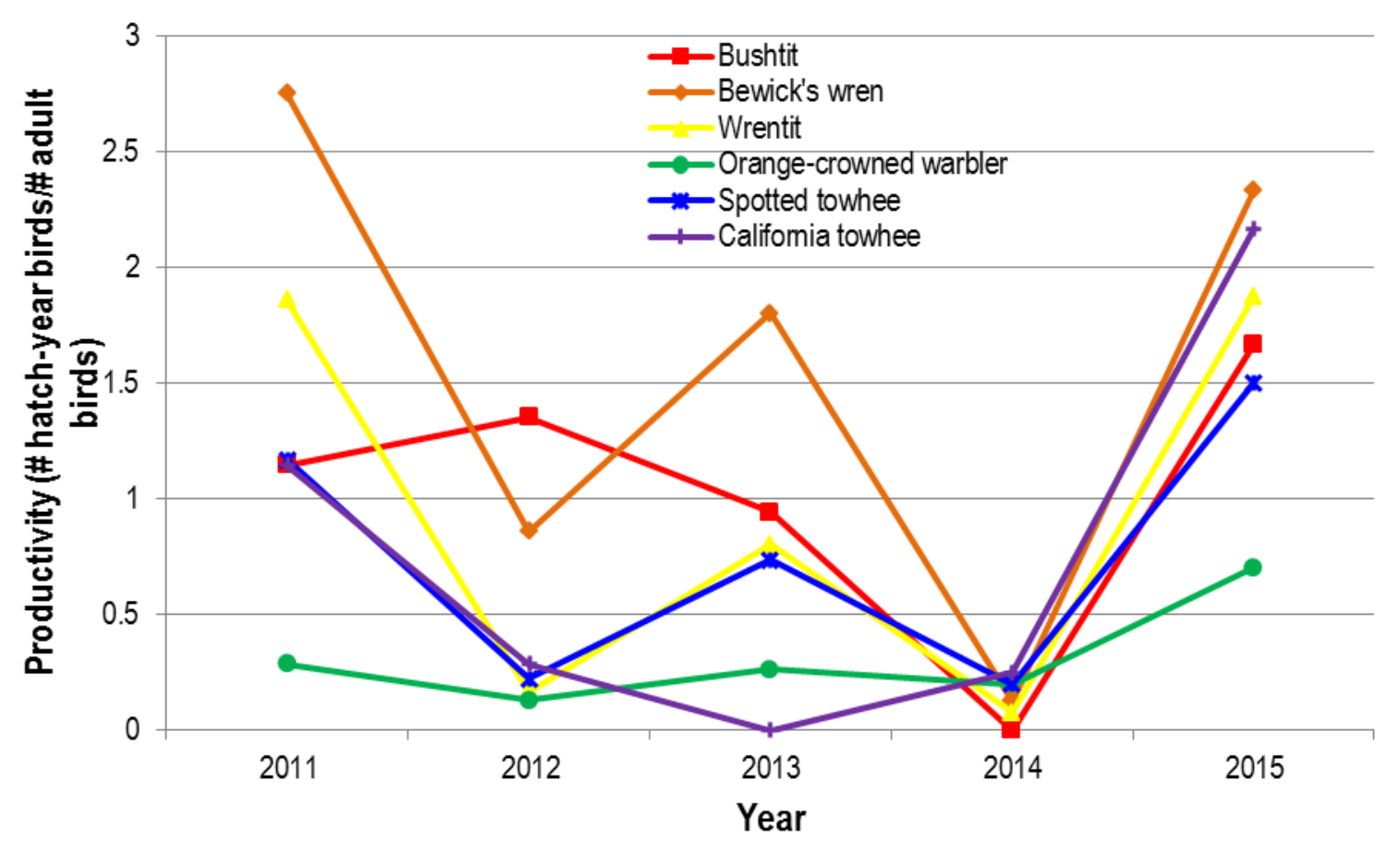

Figure C8. Annual productivity for the six most commonly captured resident species at Point Loma MAPS banding station, 2011-15. Excludes Anna's hummingbird because we could not identify individuals of this species. 

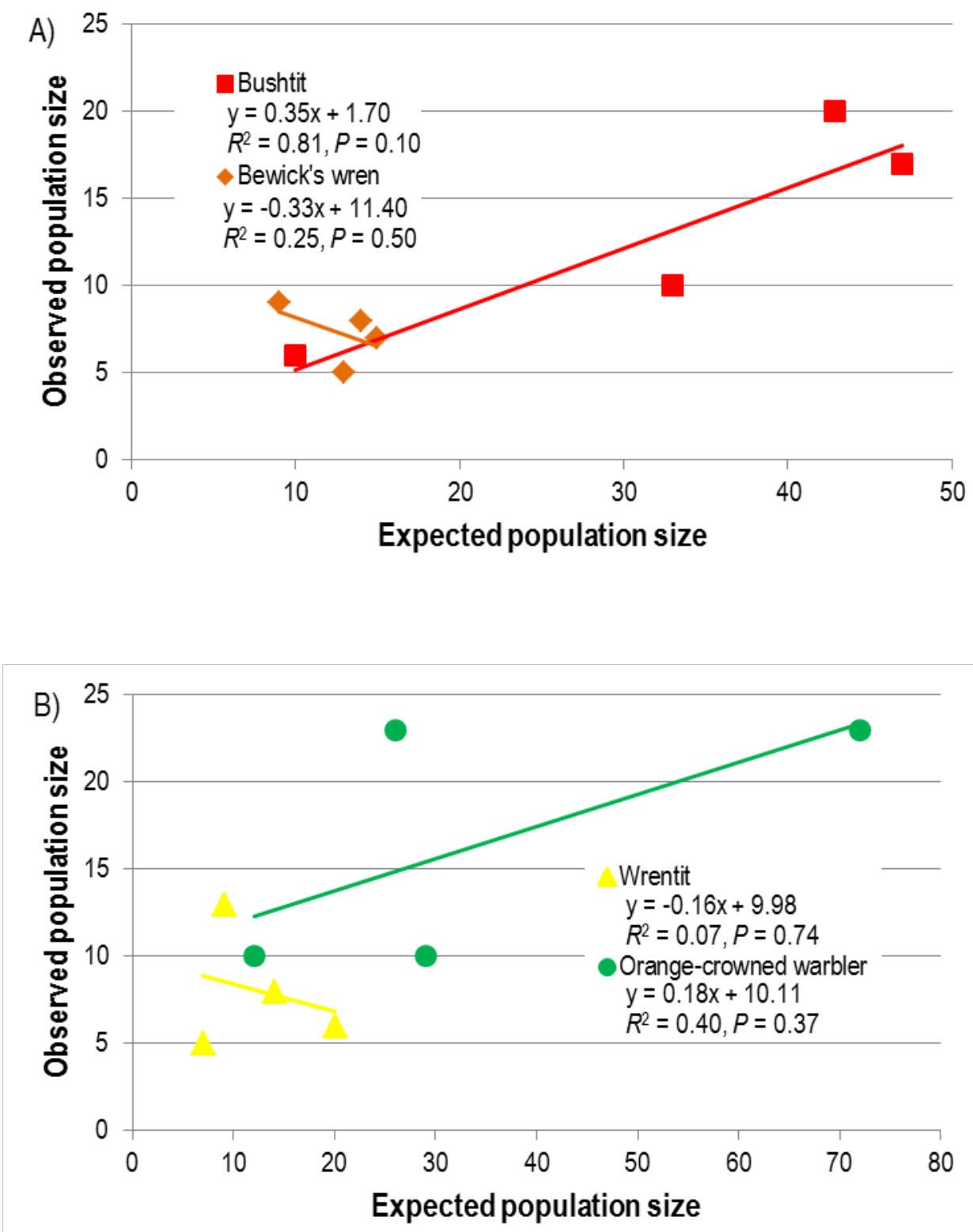

Figure C9. Observed population size of the six most commonly captured species at Point Loma MAPS, 2011-15, versus expected population size based on productivity the previous year. Productivity is measured as the ratio of hatch-year captures to adult captures of each species. 


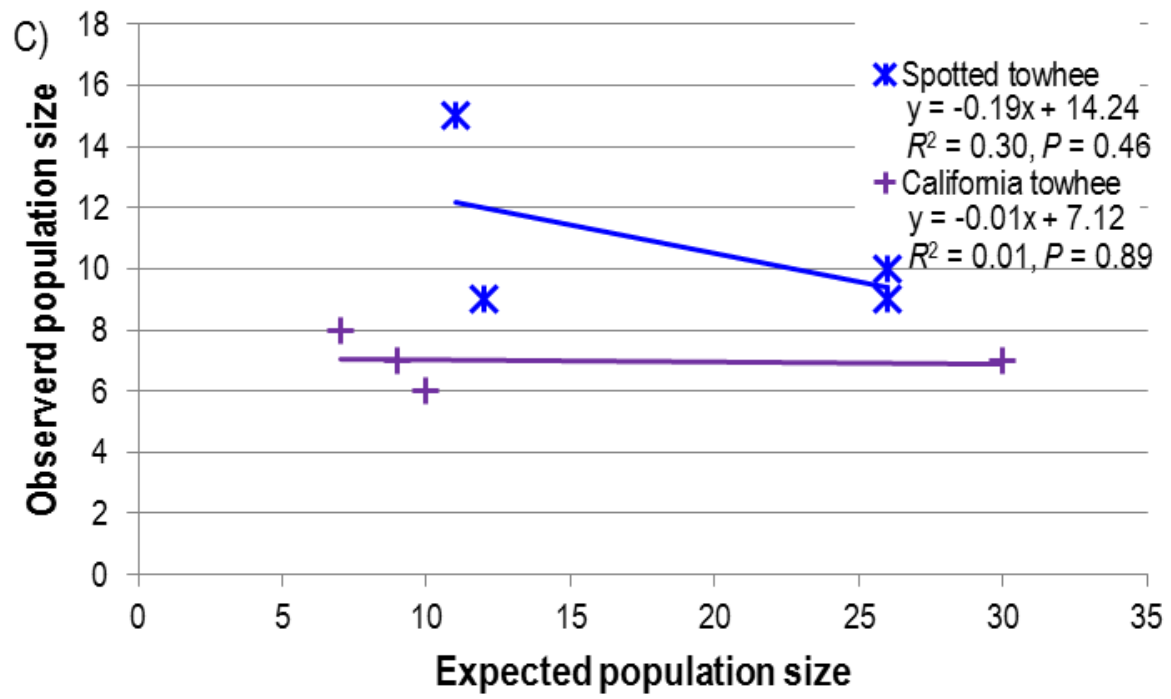

Figure C9.-Continued. 

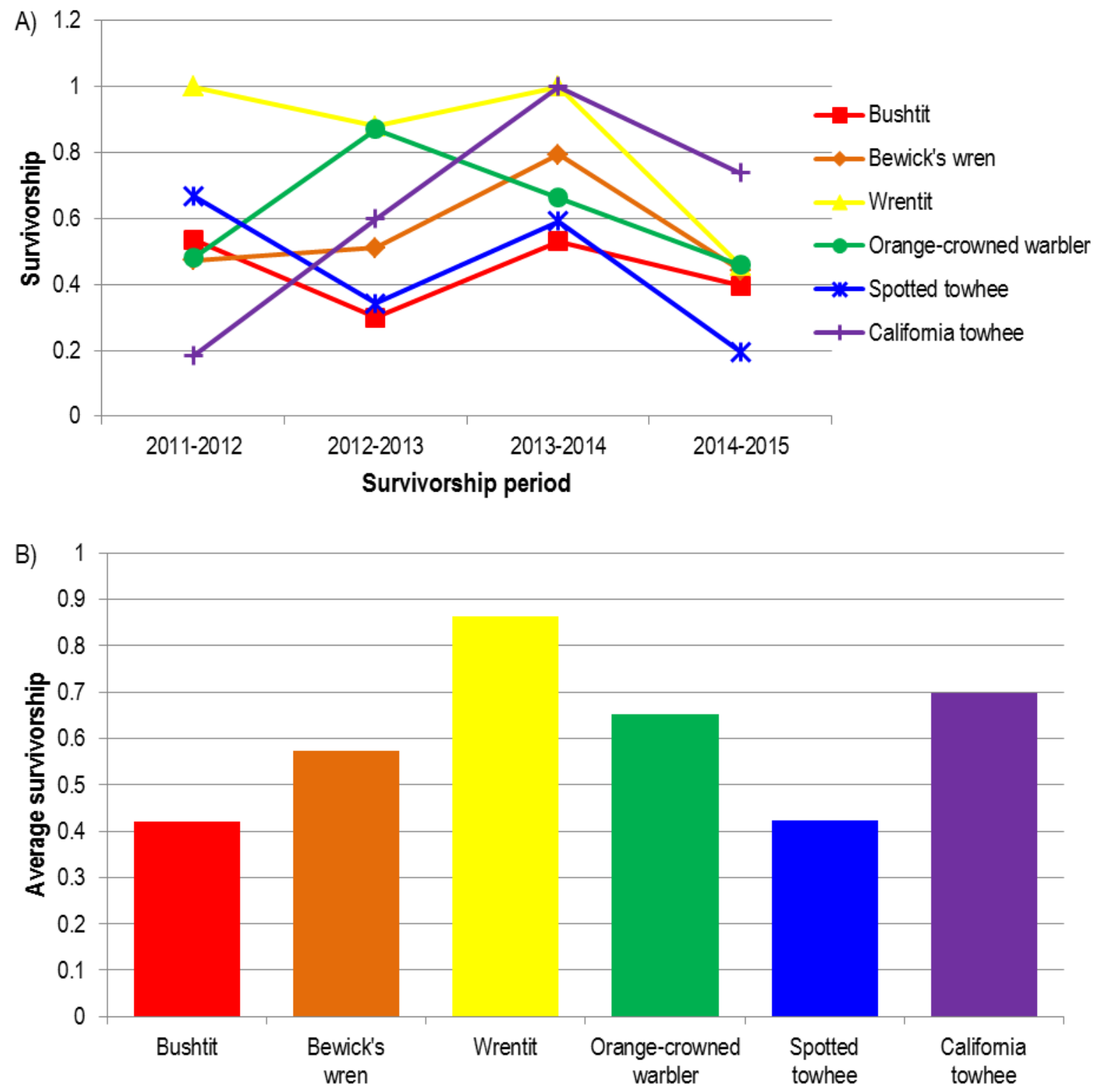

Bird species

Figure C10. Adult (a) annual survivorship (a) and (b) 5-year average survivorship calculated with Program MARK for the six most commonly captured resident species at Point Loma MAPS banding station, 2011-15. Excludes Anna's hummingbird because we could not identify individuals of this species. 

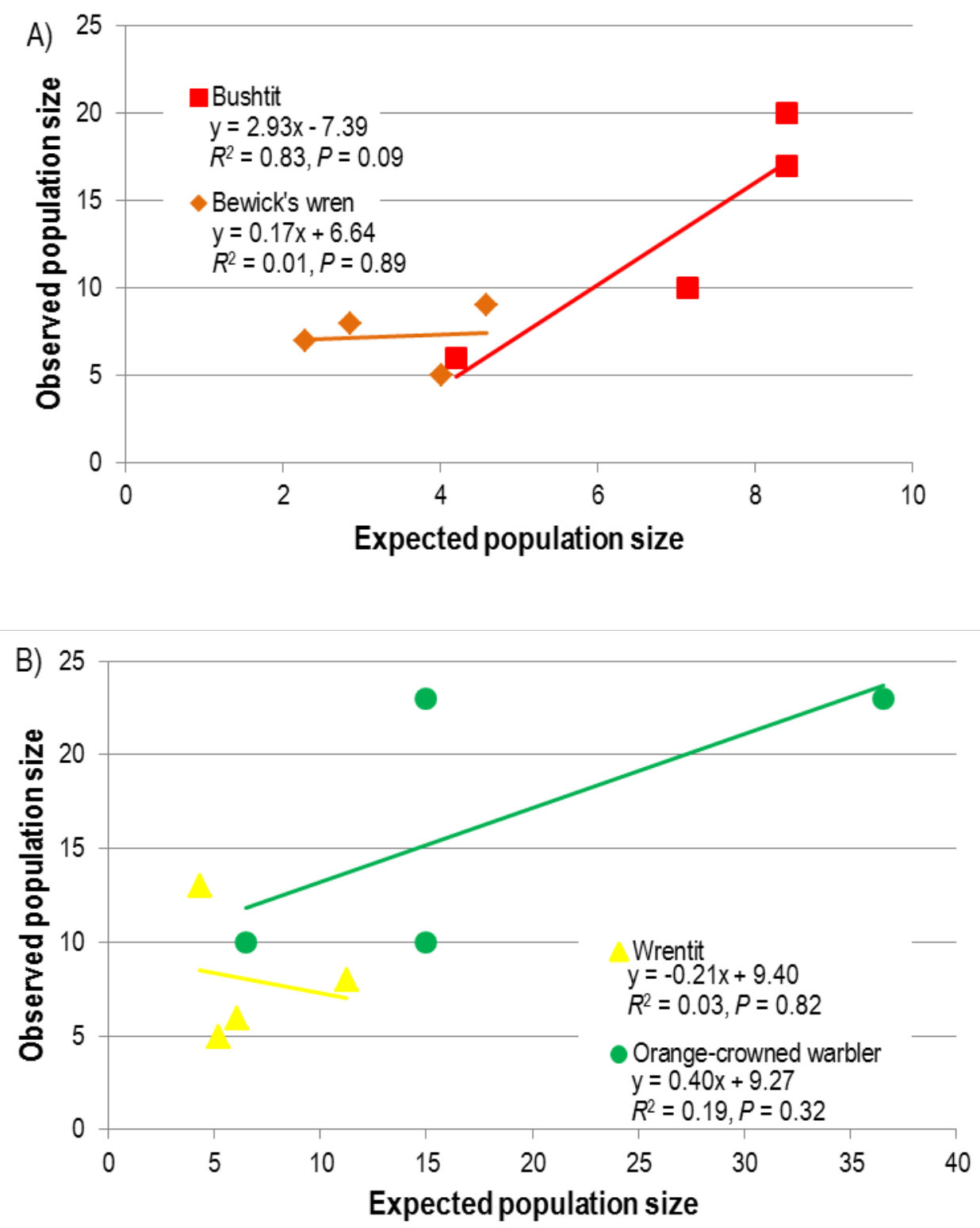

Figure C11. Observed population size of the six most commonly captured species at Point Loma MAPS, 2011-15, versus expected population size based on survivorship estimates from the previous year. Survivorship was calculated using program MARK. 


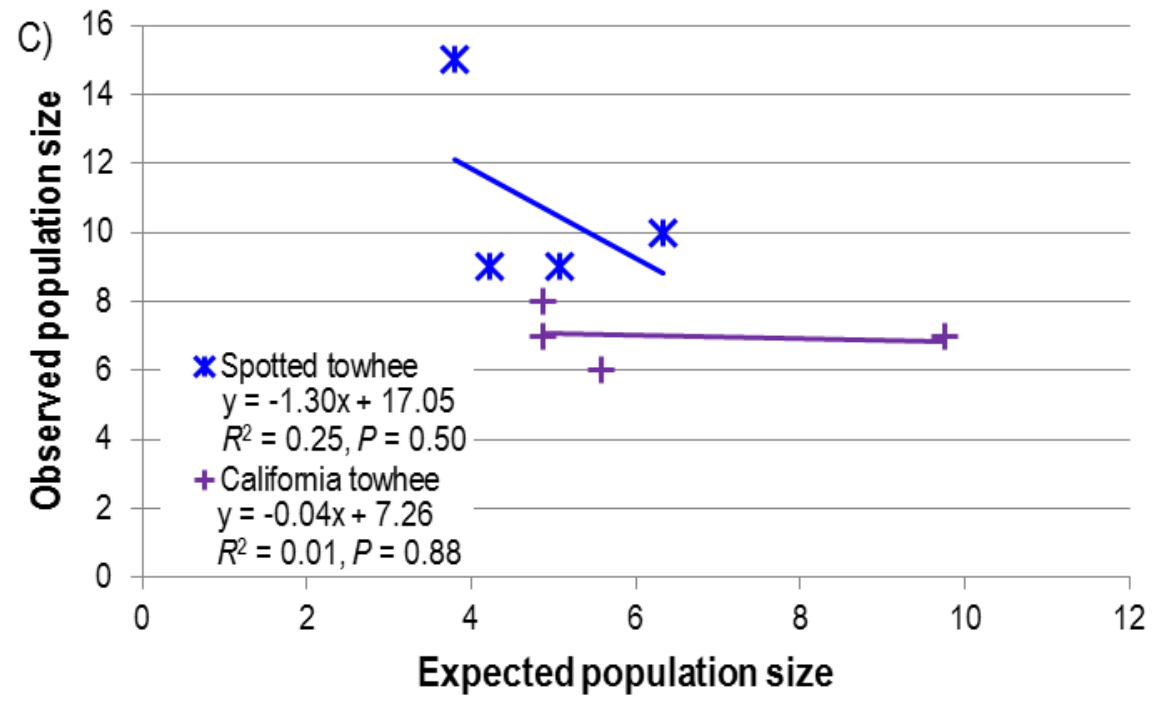

Figure C11.-Continued. 

Publishing support provided by the U.S. Geological Survey

Science Publishing Network, Tacoma Publishing Service Center

For more information concerning the research in this report, contact the Director, Western Ecological Research Center

U.S. Geological Survey

3020 State University Drive East

Sacramento, California 95819

https://www.werc.usgs.gov/ 
